\title{
Evaluation eines psychoedukativen Kurses für Angehörige von Schlaganfallpatienten
}

\author{
Dissertation zur Erlangung des akademischen Grades
}

Doktor der Philosophie

\author{
Fachbereich Rehabilitationswissenschaften der \\ Humboldt-Universität zu Berlin
}

Harriet Heier

eingereicht im Dezember 2007

Datum der Promotion: 24. 06. 2008

Erstgutachter: Prof. Dr. Ernst von Kardorff

Zweitgutachterin: Prof. Dr. Elisabeth Steinhagen-Thiessen 


\section{Widmung}

Diese Arbeit ist meinen Eltern in Liebe und Dankbarkeit gewidmet:

Meinem Vater Paul Heier (1934-2006), der in vielerlei Hinsicht Wegbereiter dieser Arbeit war. Sein Lebensmotto

Et iss wie et iss -
Et kütt wie et kütt-
Et hätt noch immer joot jejange!

begleitet mich auch nach seinem Tod.

Meiner Mutter Edith M. Heier, die mir in so vielem ein Vorbild ist - mit ihrer Herzensgüte, ihrem Humor, ihrer Lebensweisheit und ihrer bewundernswerten Fähigkeit, die Widrigkeiten des Lebens gut zu bewältigen. 


\section{Danksagung}

Diese Arbeit wäre ohne die vielfältige Unterstützung durch verschiedene Menschen nie zustande gekommen. Dafür danke ich:

Den pflegenden Angehörigen, die bereit waren, die umfangreichen Fragebögen auszufüllen und mich an ihren Lebensgeschichten teilhaben zu lassen. Die Einsichten, die ich in vielen Gesprächen gewinnen durfte, habe ich als sehr bereichernd empfunden.

Prof. Dr. Ernst von Kardorff für die wertvollen Anregungen und die wohlwollende Unterstützung sowie für die Begutachtung.

Prof. Dr. Elisabeth Steinhagen-Thiessen für die großzügige organisatorische und finanzielle Unterstützung der Arbeit sowie für die Begutachtung.

Prof. Dr. Krista Mertens für die wohlwollende Begutachtung der Arbeit.

Dr. Gernot Lämmler für die Anregung zu diesem Forschungsvorhaben, die Unterstützung bei der Planung und Durchführung der Studie, die Diskussion der Interviewanalysen sowie für die kritische Durchsicht der Arbeit.

Meinem Freund und Mentor Dr. Thomas Merten für die geduldige Wegbegleitung während aller Stadien der Arbeit. Seine vielfältigen konstruktiven Anregungen waren mir eine unschätzbare Hilfe. Ihm habe ich zu verdanken, dass ich das Ziel nie aus den Augen verloren habe.

Der Robert-Bosch-Stiftung, die das Forschungsprojekt durch ihre finanzielle Unterstützung ermöglichte.

Meinen Kolleginnen und Kollegen im Evangelischen Geriatrie Zentrum Berlin, die mich so engagiert bei der Durchführung der Angehörigenkurse unterstützt haben.

Frau Miriam Schumann für die sorgfältige und engagierte Durchführung der Interviews.

Frau Nicole Wiucha für die Transkription der Interviews.

Herrn Matthias Fleischmann für das Korrekturlesen der Interviews, die konstruktiven Diskussionen und die Beurteilung der Interviewanalysen.

Frau Beate Oberschür, die mir ab 7493 Höhenmeter des Achttausenders „Mount Dissy“ als coach zur Seite stand und mich mit Humor, liebevoller Zuwendung und Ermutigung zum Gipfelkreuz begleitet hat. Diese letzten 507 Meter waren die entscheidenden.

Meinem Freund Nils M. Becker, der mir viel über das Schreiben beigebracht hat und der mich mit zahlreichen nicht-wissenschaftlichen Schreibanlässen so herrlich von der Dissertation ablenken konnte.

Meinem Mann und wundervollen Weggefährten Bodo Heier für seine Geduld, seinen Rückhalt, seine unerschütterliche Zuversicht, seinen Humor - alle diese Jahre hindurch.

Und ganz besonders meinen Kindern Ben und Charlotte, die mir immer wieder zeigen, was jenseits von Arbeit und Leistung wirklich wichtig ist im Leben. 


\section{Inhaltsverzeichnis}

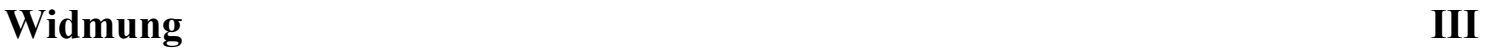

$\begin{array}{lll}\text { Danksagung IV } & \text { IV }\end{array}$

$\begin{array}{ll}\text { Zusammenfassung } & \text { XI }\end{array}$

$\begin{array}{ll}\text { Abstract } & \text { XII }\end{array}$

1. Einleitung 1

2. Theoretische Grundlagen 2

2.1. Zum Krankheitsbild Schlaganfall 2

2.1.1. Epidemiologie 2

2.1.2. Pathogenese, Ätiologie und Risikofaktoren 3

2.1.3. Symptomatik, Verlauf und Prognose 4

2.1.4. Therapie und Rehabilitation 6

2.1.5. Implikationen des Krankheitsbildes für die vorliegende Arbeit 8

2.2. Begriffsbestimmung: Pflege, Pflegebedürftigkeit, Pflegeperson 9

2.3. Demographische Aspekte der familiären Pflege 11

2.4. Belastungen durch familiäre Pflege 12

2.4.1. Entwicklungslinien der Caregiving-Forschung 13

2.4.2. Belastung von pflegenden Angehörigen: Empirische Befunde 18

2.4.3. Positive Aspekte bei der Pflege 22

2.5. Theoretischer Hintergrund der vorliegenden Arbeit 23

2.5.1. Die Kognitiv-transaktionale Stresstheorie 24

2.5.2. Das Stress-Bewältigungsmodell der familiären Pflege von Kramer (1993) 27

2.6. Weitere empirische Befunde der Angehörigenforschung vor dem Hintergrund des Stress-Bewältigungsmodell der familiären Pflege nach Kramer 29

2.6.1. Kontextuelle Aspekte der Pflegesituation 29

2.6.2. Copingressourcen 38

2.6.3. Copingstrategien 43

2.6.4. Exkurs: Zusammenhang zwischen Bewältigungsprozessen und Belastungserleben bei pflegenden Angehörigen 45

2.7. Implikationen der bisherigen empirischen Befunde für die vorliegende Arbeit 47 
2.8. Interventionsmöglichkeiten zur Verbesserung der Lebenssituation pflegender Angehöriger

2.8.1. Übersicht existierender Interventionsmöglichkeiten zur Verbesserung der Lebenssituation pflegender Angehöriger

2.8.2. Interventionsangebote für Angehörige im Kontext der Stresstheorie

2.8.3. Nutzung von Unterstützungsangeboten vs. Entlastungsbedarf

2.8.4. Evaluation von Interventionsmöglichkeiten: Empirische Befunde

2.8.5. Methodische Implikationen für die vorliegende Studie

3. Darstellung des untersuchten Interventionsprogrammes und Forschungsfragen

3.1. Eingrenzung des Untersuchungsgegenstandes - Fragestellungen und Hypothesen

3.2. Das untersuchte Interventionsprogramm: Psychoedukativer Kurs für pflegende Angehörige von Schlaganfallbetroffenen

4. Methode

4.1. Wissenschaftstheoretische Aspekte quantitativer und qualitativer Methoden

4.2. Methodische Standortbestimmung und Implikationen für die vorliegende Studie

5. Methode - Quantitative Untersuchung

5.1. Allgemeines Studiendesign

5.2. Stichprobe

5.3. Rücklaufquote

5.4. Messinstrumente

$\begin{array}{lll}\text { 5.5. } & \text { Statistische Auswertung } & 106\end{array}$

6. $\quad$ Ergebnisse - Quantitative Untersuchung 107

6.1. Beeinträchtigungen des psychischen und körperlichen Wohlbefindens der Angehörigen

6.2. Veränderung des Informiertheitsgrades in Bezug auf Schlaganfall-spezifisches Wissen

6.3. Nutzung (psycho-)sozialer Entlastungsangebote

6.4. Subjektiv erlebter Nutzen des Kurses 
7. Methode - Qualitative Untersuchung 115

$\begin{array}{ll}\text { 7.1. Stichprobe } & 115\end{array}$

$\begin{array}{ll}\text { 7.2. Untersuchungsablauf } & 116\end{array}$

7.3. Das Leitfaden-Interview und die erhobenen Variablen 117

7.4. Fähigkeiten und Training der Interviewerin 118

7.5. Erfassung und Auswertung der Interviewtranskripte 120

$\begin{array}{lll}\text { 8. } & \text { Ergebnisse - Qualitative Untersuchung } & 128\end{array}$

8.1. Die ermittelten Bewältigungsstrategien 128

8.2. Gesamtbeurteilung des Bewältigungsverhaltens 142

8.3. Vertiefende Fallanalysen 143

8.3.1. Frau T.: „Man will doch gar nichts Besonderes, man will ja nur mal eben frei sein.“ 145

8.3.2. Frau M.: „Man muss einfach mit dem Hier, Heute und Jetzt umgehen lernen“ 160

$\begin{array}{lll}9 . & \text { Diskussion } & \mathbf{1 8 0}\end{array}$

9.1. Quantitative Befunde 180

9.2. Qualitative Befunde 186

9.3. Überlegungen zur Forschungsmethodik: Plädoyer für eine Kombination quantitativer und qualitativer Methoden 191

9.4. Konsequenzen für die Forschung 192

9.4.1. Implikationen für zukünftige Studien zum Thema Caregiving 193

9.4.2. Implikationen für zukünftige Interventionsstudien 194

9.5. Implikationen für die Praxis der Angehörigenarbeit: systematische und zugehende Beratung der pflegenden Angehörigen 197

9.6. Ausblick 204

$\begin{array}{ll}10 \text { Literatur } & 207\end{array}$

Anhang A: Materialien für die quantitative Untersuchung 231

Anhang B: Materialien für die qualitative Untersuchung 253 


\section{Abbildungsverzeichnis}

Abbildung 1: Ein theoretischer Rahmen zur Betrachtung von Stressbewältigung in der familiären Pflege (nach Kramer, 1993)

Abbildung 2: Interventionsmöglichkeiten zur Verbesserung der Lebenssituation von pflegenden Angehörigen

Abbildung 3: Wirkweise der Interventionsansätze im Kontext der Stresstheorie (modifiziert nach Kahana \& Kinney, 1991)

Abbildung 4: Wirkweise des psychoedukativen Kurses im Rahmen des

Stressbewältigungsmodells (nach Kramer, 1993)

Abbildung 5: Globale subjektive Belastung zu den Messzeitpunkten 1, 2, 3 für parallelisierte Stichproben (pro Gruppe $\mathrm{N}=26$ )

Abbildung 6: Nutzung psychosozialer Entlastungsangebote zu den Messzeitpunkten 1, 2 und 3 für parallelisierte Stichproben ( $\mathrm{N}=26$ pro Gruppe)

Abbildung 7: Subjektiv erlebter Nutzen des Angehörigenkurses für $N=42$ 


\section{Tabellenverzeichnis}

Tabelle 1: Charakteristika der Gesamtstichprobe zum Messzeitpunkt 1

Tabelle 2: Charakteristika der pflegenden Angehörigen (parallelisierte Teilstichproben) zum Messzeitpunkt 1

Tabelle 3: Charakteristika der Patienten (parallelisierte Teilstichproben) zum Messzeitpunkt 1

Tabelle 4: Rücklaufquote zu den Messzeitpunkten (MZP) 1, 2, 3

Tabelle 5: Verwendete Untersuchungsverfahren: Pflegende Angehörige

Tabelle 6: Verwendete Untersuchungsverfahren: Pflegerelevante Kennzeichen der Patienten

Tabelle 7: Psychisches und körperliches Befinden der pflegenden Angehörigen für Messzeitpunkte 1, 2 und 3, $\mathrm{N}=26$ pro Gruppe

Tabelle 8: Ausmaß des Schlaganfallspezifischen Wissens der pflegenden Angehörigen für Messzeitpunkte 1, 2 und 3, $\mathrm{N}=26$ pro Gruppe

Tabelle 9: Von Angehörigen genannte Veränderungen in der Pflegesituation aufgrund der Teilnahme am Kurs

Tabelle 10: Von Angehörigen genannte positive und hilfreiche Aspekte des Kurses

Tabelle 11: Beschreibung der qualitativen Stichprobe anhand ausgewählter quantitativer Variablen

Tabelle 12: Kategoriensystem der Bewältigungsstrategien

Tabelle 12 Fortsetzung

Tabelle 12 Fortsetzung 
Tabelle 13: Häufigkeit der Nennungen der verwendeten Bewältigungsstrategien

Tabelle 14: Gesamtbeurteilung des Bewältigungsverhaltens der pflegenden Angehörigen

Tabelle 15: Belastungswerte der pflegenden Angehörigen zu den Messzeitpunkten 1 und 4 und Gesamtbeurteilung des Bewältigungsverhaltens

Tabelle 16: Ausprägung von Depression, Angst und körperlichen Beschwerden zu den 4 Messzeitpunkten bei Frau T.

Tabelle 17: Ausprägung von Depression, Angst und körperliche Beschwerden zu den 4 Messzeitpunkten bei Frau M. 


\section{Zusammenfassung}

Obwohl mittlerweile gesichert ist, dass Angehörige von Patienten mit zerebralen Schädigungen hochgradig belastet sind, findet in den meisten Rehabilitationseinrichtungen keine systematische Angehörigenarbeit statt. Im vorliegenden Forschungsprojekt wurde ein psychoedukativer Kurs für Angehörige von Schlaganfallpatienten entwickelt und in einem Längsschnitt-Kontrollgruppen-Design mit 3 Messzeitpunkten $(N=52)$ evaluiert. Ergänzend $\mathrm{zu}$ den quantitativen Erhebungsverfahren wurden im Rahmen einer qualitativen Studie auch Interviewdaten erhoben und analysiert. In der quantitativen Studie kamen als Messinstrumente Selbstbeurteilungsverfahren zu Depression, Angst und körperlichen Beschwerden zum Einsatz. Außerdem wurden die globale subjektive Belastungswahrnehmung, das Schlaganfall-spezifische Wissen, die Nutzung psychosozialer Entlastungsangebote und der subjektiv erlebte Nutzen des Kurses erhoben. Die Kursteilnehmer zeigten im Gegensatz zur Kontrollgruppe eine signifikante Zunahme des Schlaganfall-spezifischen Wissens und nutzten mehr Entlastungsangebote. Die subjektive Belastung nahm nur bei der Treatmentgruppe im zeitlichen Verlauf ab, während sie bei der Kontrollgruppe konstant blieb. Der Nutzen des Kurses wurde von den Kursteilnehmern als sehr hoch eingeschätzt. In den standardisierten Skalen zum psychischen und körperlichen Befinden konnten allerdings in keiner der beiden Gruppen signifikante Veränderungen im zeitlichen Verlauf festgestellt werden. Dies wird u. a. darauf zurückgeführt, dass emotionale Verarbeitungsmuster resistenter gegenüber Veränderungen sind als Verhaltenskomponenten. Mit einer Teilstichprobe von elf Angehörigen wurden im Rahmen des qualitativen Untersuchungsteils Interviews durchgeführt. In vertiefenden Fallanalysen wurden die Formen und Strategien der Bewältigung analysiert. Außerdem wurde untersucht, inwiefern sich der bisherige Bewältigungsstil moderierend auf die Effekte des Kurses auf das Belastungserleben der Angehörigen auswirkte.

Hier zeigte sich, dass der Kurs Angehörige mit einem bereits funktionalen Bewältigungsstil weiter $\mathrm{zu}$ stabilisieren vermag. Bei Angehörigen mit eher dysfunktionalem Bewältigungsstil muss das Veränderungspotential hinsichtlich der Reduzierung des Belastungserlebens auf niedrigerer Ebene angesiedelt werden. 
Schlüsselwörter: pflegende Angehörige, Schlaganfall, Interventionsprogramme für Angehörige, Evaluation

\begin{abstract}
Although numerous studies have shown that caregivers of patients with cerebral diseases are highly burdened, most rehabilitation clinics do not offer systematic intervention programs for caregivers. A psychoeducative seminar for carers of stroke patients was developed and evaluated by means of a pre-post control group design with fifty-two subjects. Self-rating scales measuring depression, anxiety and physical complaints were employed. Additionally, subjective burden, knowledge regarding stroke, use of social services and subjective gains resulting from the seminar were measured. The treatment group showed a significant increase in their knowledge regarding stroke and used more social services which was not found for the control group. Also, only the treatment group revealed a decrease in subjective burden. High satisfaction with the seminar was reported by all members of the treatment group. However, concerning the standardized instruments measuring psychological and physical well-being, no significant changes were found for both groups. One reason for this finding might be that emotional processes are more resistant to changes than behavioral aspects. In addition to the quantitative data interviews were carried out with 11 caregivers from the original sample and analysed by means of qualitative methods. It was analysed which coping-strategies caregivers used and in which way the copingstyle moderated the effects of the intervention program. The in-depth case-studies revealed that carers with a generally more functional coping style were further stabilized. Caregivers with a more dysfunctional coping-style previous to the intervention showed less potential for change regarding the reduction of stress-levels. However, changes were observed concerning their ability and willingness to employ services.
\end{abstract}

Keywords: Caregivers, stroke, intervention programs for caregivers, evaluation 


\section{Einleitung}

Dank der Fortschritte in der Medizin versterben immer weniger Menschen in den westlichen Industrieländern an Erkrankungen infektiöser Ursache. Dagegen nehmen chronische Erkrankungen stark zu. Mit jährlich mehr als 150.000 erstmaligen Schlaganfällen und einer Million betroffener Bundesbürger stellt das Krankheitsbild Schlaganfall neben den Demenzerkrankungen eine der bedeutendsten Herausforderungen für das deutsche Gesundheitssystem der Gegenwart dar. Die Überlebenschancen Schlaganfallerkrankter haben sich in den vergangenen Jahrzehnten erheblich verbessert, sodass ca. $60 \%$ der Betroffenen das Akutereignis überleben. Allerdings bleiben $40 \%$ dauerhaft beeinträchtigt und benötigen Hilfe oder Pflege (Diener, Hacke \& Forsting, 2004). Die Mehrheit der Schlaganfallbetroffenen kehrt nach Aufenthalt im Akutkrankenhaus oder einer Rehabilitationseinrichtung nach Hause zurück. In erster Linie sind es die Angehörigen, die oft über Jahre hinweg die Pflege des kranken Familienmitgliedes übernehmen. Sie gewährleisten durch ihr Engagement den Vorrang häuslicher Pflege vor stationärer Versorgung. Dies entspricht sowohl dem Wunsch der meisten Betroffenen, dem Anspruch im fachlichen Altenhilfediskurs als auch den Prinzipien des Pflegeversicherungsgesetzes. In den meisten Fällen liegt die Hauptverantwortung für die Pflege bei einer Person. Pflegende Angehörige sind gravierenden Belastungen ausgesetzt, sowohl zeitlicher, emotionaler, körperlicher, finanzieller als auch familiärer Art. Dennoch existieren in Deutschland kaum Untersuchungen, die sich systematisch mit Interventionskonzepten für Angehörige von Schlaganfallpatienten beschäftigen.

Zentrale Fragestellung der vorliegenden Dissertation ist daher, ob die Teilnahme an einem psychoedukativen Kurs ein wirksames Interventionsangebot für Angehörige von Schlaganfallpatienten darstellt. Zur Überprüfung der Effektivität des Angehörigenkurses wurden verschiedene Indikatoren psychischer und körperlicher Belastung in einem Längsschnitt-Kontrollgruppen-Design mit 3 Messzeitpunkten erhoben. Die quantitative Untersuchung wurde durch einen qualitativen Teil ergänzt, bei der Angehörige in Interviews zu ihren Strategien der Bewältigung ihrer Lebenssituation und zu dem Nutzen der Kursteilnahme befragt wurden. 


\section{Theoretische Grundlagen}

Einführend wird im theoretischen Teil der vorliegenden Arbeit zunächst das Krankheitsbild Schlaganfall vorgestellt. Dies soll die objektiven Rahmenbedingungen veranschaulichen, mit denen Angehörige von Schlaganfallpatienten konfrontiert sind. Im Anschluss wird ein Überblick über die Forschungsliteratur zur Situation von pflegenden Angehörigen gegeben. Dabei werden zunächst die Entwicklungslinien des Forschungsgebietes nachgezeichnet. Ein weiterer Abschnitt beschäftigt sich mit empirischen Studien, die die Auswirkungen der familiären Pflege auf die Angehörigen untersuchen. Es folgt die Darstellung eines theoretischen Modells zu Stress und Stressverarbeitung sowie die Adaptation des Modells auf die Situation der familiären Pflege. Weitere empirische Befunde der Angehörigenforschung werden auf der Grundlage dieses Modells vorgestellt.

Das darauffolgende Kapitel bietet einen Überblick über die bislang entwickelten Interventionsmöglichkeiten zur Verbesserung der Lebenssituation pflegender Angehöriger. Außerdem werden Untersuchungen vorgestellt und diskutiert, die sich mit der Evaluation von Interventionskonzepten befassen. Den Abschluss des Theorieteils bilden eine Eingrenzung des Untersuchungsgegenstandes der vorliegenden Arbeit und eine Beschreibung der Hypothesen und Fragestellungen.

\subsection{Zum Krankheitsbild Schlaganfall}

\subsubsection{Epidemiologie}

Unter dem Begriff Schlaganfall oder zerebraler Insult ist eine schlagartig eingetretene Funktionsstörung eines Teils des Gehirns zu verstehen, die mindestens über 24 Stunden besteht. Zerebrovaskuläre Erkrankungen sind nach kardiovaskulären Störungen und Neoplasien die dritthäufigste Todesursache in Deutschland (Gleixner, Müller \& Wirth, 1999). Sie stellen außerdem die Hauptursache für eine chronische Behinderung bei Erwachsenen dar (Kolominsky-Rabas et al., 2006). Schätzungen der Neuerkrankungsrate in Deutschland variieren zwischen 120.000 und 350.000 auftretende Schlaganfälle pro Jahr (Berger, Kolominsky-Rabas, Heuschmann \& Keil, 
2000; Diener, Hacke \& Forsting, 2004). Der zerebrale Insult betrifft überwiegend ältere Menschen. Mit zunehmendem Alter ist ein exponentieller Anstieg der Erkrankung zu verzeichnen. In der Altersgruppe der 65- bis 74-jährigen liegt die Inzidenz bei 100.000 pro Jahr. Bei über 75-jährigen ist die Inzidenz mehr als 100mal höher als bei 25- bis 34jährigen (Häussler, 1996), bei den über 84-jährigen sogar mehr als 500mal höher (Kolominsky-Rabas et al. 1998). Hinsichtlich der Prävalenz wird geschätzt, dass in Deutschland insgesamt ca. 1 bis 1,5 Millionen Menschen an den Folgen eines Schlaganfalls leiden; diese Zahlen entsprechen den europäischen Vergleichswerten (Berger et al., 2000).

\subsubsection{Pathogenese, Ätiologie und Risikofaktoren}

Schlaganfälle sind in ca. $85 \%$ auf eine Mangeldurchblutung (zerebrale Ischämie) zurückzuführen, in $10 \%$ auf eine zerebrale Blutung (hämorrhagischer Infarkt) und in $5 \%$ auf eine Blutung unter die Hirnhaut (Subarachnoidalblutung) (Diener, Hacke \& Forsting, 2004). Bei einer zerebralen Ischämie kommt es meist durch einen Gefäßverschluss zu einer akuten Minderversorgung eines Teils des Gehirns mit Blut und damit auch zu einer Unterversorgung mit Sauerstoff und Energieträgern (Glukose). Ursachen für eine solche Minderversorgung können arterio-arterielle und kardiale Embolien, lokale Thrombosen eines arteriosklerotisch geschädigten Gefäßes und seltener Gerinnungsstörungen sein. Bei einer intrazerebralen Blutung kommt es zu einer Ruptur eines Gefäßes. Als Ursache kommen eine arterielle Hypertonie, Gefäßmissbildungen, Gerinnungshemmung (durch Medikamente) oder Tumor- und Metastaseneinblutungen in Betracht (Weiller, 1996).

Das individuelle Risiko, einen Schlaganfall zu erleiden, ist stark vom Alter abhängig und verdoppelt sich alle zehn Jahre ab dem 55. Lebensjahr (Bamford et al., 1988). Neben dem Lebensalter sind verschiedene spezifische Risikofaktoren von Bedeutung. Dazu gehören arterielle Hypertonie, Hypercholesterinämie, Diabetes mellitus, koronare Herzkrankheit, Nikotinkonsum, Alkoholkonsum, sowie Übergewicht (Steinhagen-Thiessen, Gerok \& Borchelt, 1992). Bei Frauen gilt die Einnahme oraler Kontrazeptiva in Kombination mit Nikotinkonsum als weiteres Risiko. Insbesondere bei jüngeren Patienten gewinnt Drogenkonsum als weiterer Risikofaktor zunehmend an 
Bedeutung (Sloan, Kittner, Rigamont \& Price, 1991).

Liegen mehrere Risikofaktoren vor, nimmt das Schlaganfallrisiko nicht linear, sondern exponentiell zu. Für jeweils ein Drittel aller Schlaganfälle können die Risikofaktoren Bluthochdruck, Nikotinkonsum und Hypercholesterinämie verantwortlich gemacht werden (Diener, Hacke \& Forsting, 2004).

\subsubsection{Symptomatik, Verlauf und Prognose}

Die Symptomatik nach einem Schlaganfall ist vielfältig. Die häufigsten Beeinträchtigungen sind Halbseitenlähmung (Hemiplegie), Einschränkung oder Verlust der Blasen- und Darmkontrolle (Inkontinenz), Störungen des Schluckens und/oder der Artikulation (Dysphagie, Dysarthrie), Beeinträchtigungen der Sprache (Aphasie) sowie Einschränkungen des Gesichtsfeldes (Hemianopsie). Außerdem sind drei Monate nach dem Schlaganfallereignis in $30 \%$ der Fälle neuropsychologische Störungen zu beobachten (Serrano, Domingo, Rodriguez-Garcia, Castro \& del Ser, 2007). Dabei können die Bereiche Aufmerksamkeit, Gedächtnis und Lernen, visuell-räumliche Wahrnehmung und exekutive Funktionen wie Problemlösen und Planen beeinträchtigt sein (Denzler, 1994a, 1994b). Zwei Jahre nach dem Schlaganfallereignis liegen bei ca. $40 \%$ der Betroffenen neuropsychologische Beeinträchtigungen vor, die jedoch nicht die Kriterien einer vaskulären Demenz erfüllen (Serrano et al., 2007).

Als Folge des Schlaganfalls kann es weiterhin zu Veränderungen der Stimmung, der Persönlichkeit und des Verhaltens kommen. Die häufigste Veränderung im Bereich des Affektes ist die Depression. Die Häufigkeitsraten bewegen sich zwischen 30 bis $40 \%$ (Upton \& Finlayson, 1987) und 50 bis $70 \%$ (Robinson, Starr, Kubos \& Price, 1983). Persönlichkeitsveränderungen zeigen sich bei Schlaganfallbetroffenen in vielen Fällen außerdem in Form von gesteigerter Ängstlichkeit, impulsivem Verhalten, Aggressivität, veränderter sozialer Wahrnehmung und inadäquater Krankheitseinsicht (Binder, 1983; Denzler, 1994b). Trotz der Häufigkeit des Schlaganfalls bei älteren Menschen mangelt es insbesondere an neueren epidemiologischen Daten zu den oft schwerwiegenden Folgen des Schlaganfalls. 
In Bezug auf die Auswirkungen der Beeinträchtigungen auf die selbständige Lebensführung ist davon auszugehen, dass $47 \%$ der Betroffenen nach sechs Monaten unabhängig von fremder Hilfe leben können. $12 \%$ sind als mittelgradig und $32 \%$ als leichtgradig beeinträchtigt einzustufen. $9 \%$ gelten als schwer beeinträchtigt. Jedoch sind bei älteren Patienten schlechtere Werte vorauszusetzen, da diese Daten für ein bevölkerungsrepräsentatives Patientenkollektiv berechnet wurden (Wade \& Hewer, 1987b).

Die Prognose in Bezug auf das Überleben und auf den Rehabilitationserfolg wird insbesondere von folgenden Faktoren negativ beeinflusst: ein hohes Lebensalter, ein lang anhaltendes initiales Koma, das Auftreten schwerer motorischer Störungen, eine gestörte Auffassungsgabe und häufige frühere Insulte (Schütz, 1994). Der bedeutsamste Prädiktor für die Frühmortalität (3 Wochen) ist der Grad der Bewusstseinsstörung. Als wichtigste Prädiktoren für die Sterberate innerhalb des ersten Jahres gelten Lebensalter, kardiovaskuläre Risikofaktoren, das Vorliegen einer initialen Urininkontinenz und ein Barthel-Index unter 25 innerhalb der ersten Woche nach dem Schlaganfall (Hesse, 1994; Meins, Meier-Baumgartner, Neetz \& von Rentelen-Kruse, 2002). Die Letalitätsrate nach Ablauf des ersten Jahres nach dem Schlaganfallereignis ist zudem vom Lebensalter des Betroffenen abhängig. Sie variiert zwischen $10 \%$ für unter 60-jährige und $50 \%$ für über 80-jährige. Insgesamt ist die Letalität in den ersten vier Wochen nach dem Ereignis am größten: Innerhalb dieses Zeitraumes versterben $20 \%$ der Patienten, 40 \% versterben im ersten Jahr (Kolominsky-Rabas et al., 1998). In den letzten 20 Jahren ist jedoch ein stetiger Rückgang der Mortalität zu beobachten. Dies ist vor allem auf die verbesserte Behandlung von Komplikationen wie Pneumonien oder Lungenembolien sowie auf die zunehmende Implementierung von SchlaganfallSpezialstationen zurückzuführen (Gleixner, Müller \& Wirth, 1999; Leys, Ringelstein, Kaste \& Hacke, 2007).

Für den kurzfristigen Rehabilitationsverlauf im Akutkrankenhaus sind kognitive Faktoren prognostisch weniger ausschlaggebend. Allerdings gewinnen sie nach Ablauf von einigen Monaten zunehmend an Bedeutung und determinieren entscheidend die Alltagsbefähigung (Mobilität und Alltagsaktivitäten) eines Patienten (Denzler, 1994b). Generell ist davon auszugehen: je schwerer die initialen Beeinträchtigungen sind, desto 
schwerwiegender ist die Behinderung 6 Monate später (Wade, 1993). Grundsätzlich ist innerhalb der ersten 4 Wochen die größte Remission der schlaganfallbedingten Ausfälle zu erwarten, aber auch bis zu 6 Monaten nach Akutereignis sind noch Verbesserungen möglich. Über diesen Zeitraum hinaus sind laut Wade (1993) zwar in Einzelfällen noch bedeutsame Fortschritte zu verzeichnen, sie bilden jedoch eher die Ausnahme.

\subsubsection{Therapie und Rehabilitation}

In den letzten Jahren ist durch die bundesweite Einrichtung von SchlaganfallSpezialstationen, so genannten Stroke Units, eine Optimierung in der Akutphase der Schlaganfallbehandlung zu verzeichnen, wenngleich eine optimale Versorgungssituation noch nicht flächendeckend gewährleistet ist. Inzwischen liegen außerdem Behandlungsleitlinien der Deutschen Gesellschaft für Neurologie vor, die in der Akutphase evidenzbasierte Standards für die Therapie der medizinischen Ursachen des Schlaganfalls vorgeben (Diener \& Hacke, 2002). Aber auch die Leitlinienorientierte Akutbehandlung wird noch nicht überall umgesetzt (Leys, Ringelstein, Kaste \& Hacke, 2007). Durch die Versorgung in einer Stroke Unit kann die Letalität in den ersten Wochen um über 50 Prozent reduziert werden. Auch der Langzeitverlauf über fünf Jahre wird hinsichtlich Behinderungsgrad und Pflegebedürftigkeit deutlich positiv beeinflusst (Stroke Unit Triallists' Collaboration, 1997).

Der Schwerpunkt der Therapie nach der Akutphase besteht einerseits in der konsequenten Minimierung der Risikofaktoren sowie andererseits in der rehabilitativen Behandlung. Ziel der Rehabilitation liegt in der „Selbstbestimmung und Teilhabe am sozialen Leben“, was auch vom Gesetzgeber mit der Verabschiedung des Sozialgesetzbuches IX im Juli 2001 explizit festgeschrieben wurde. Dem zugrunde liegt das Krankheitsfolgemodell mit der Internationalen Klassifikation der Funktionsfähigkeit, Behinderung und Gesundheit (ICF) der Weltgesundheitsorganisation (WHO) das ebenfalls 2001 verabschiedet wurde (WHO, 2001). Daraus ergibt sich, dass in der Rehabilitation nicht nur eine Verbesserung von Funktionen - im Sinne einer Behebung oder Milderung der Krankheitssymptome - im Fokus der therapeutischen Interventionen steht, sondern ebenso die Bewältigung oder 
der Abbau von krankheitsbedingten, innerpsychischen oder äußeren Behinderungen, die dem Ziel der Selbstbestimmung und der Teilhabe am sozialen Leben im Wege stehen können. Dies erfordert, die Schlaganfallfolgen zwingend im Kontext der biographischpersönlichkeitsabhängigen, der sozialen und gegebenenfalls der beruflichen Bedingungen $\mathrm{zu}$ beurteilen und $\mathrm{zu}$ behandeln. In der Praxis der Rehabilitationseinrichtungen gelten damit schon eine partielle Restitution mit der Möglichkeit zur Fortführung des Lebens im gewohnten Umfeld und das Wiedererlangen größtmöglicher Selbständigkeit als Rehabilitationserfolg (Lenzen-Großimmlinghaus \& Steinhagen-Thiessen, 2000).

Die Bandbreite und die Komplexität der möglichen Defizite nach einem Schlaganfall erfordern ein interdisziplinäres Rehabilitationsteam, bestehend aus Ärzten, Pflegekräften, Physiotherapeuten, Ergotherapeuten, Neuropsychologen, Logopäden sowie Sozialarbeitern (Nehen, 1998). Mittlerweile liegen zahlreiche Studien vor, die den Erfolg rehabilitativer Behandlung nach einem Schlaganfall nachweisen, der sich in signifikanten Verbesserungen der Mobilität und der Autonomie der Patienten und in einem über mehrere Jahre anhaltenden Therapieerfolg niederschlägt (z. B. Langhammer, 2007; Mauritz, 2004; Meier-Baumgartner, Blum, Haas \& Meins, 2000). Auch die Forschungsergebnisse zur Plastizität des Gehirns verdeutlichen die Wichtigkeit einer umfassenden Rehabilitation (Boniface \& Ziemann, 2003; Nelles, 2004; Seitz \& Bütefisch, 2006).

Die Bundesarbeitsgemeinschaft für Rehabilitation (BAR) propagiert ein abgestuftes Versorgungssystem mit stationären, teilstationären und ambulanten Elementen (BAR, 2005). Wenn im stationären Bereich die Voraussetzungen für ein Leben im eigenen häuslichen Rahmen erreicht sind, sollte idealerweise die rehabilitative Behandlung ambulant und wohnortnah weitergeführt werden. Denn die sich aus den Schlaganfallfolgen ergebenden Behinderungen zeigen sich in ihrem Ausmaß und ihrer Bedeutung für den Betroffenen und seine Angehörigen oft erst, wenn der Patient wieder in seinem häuslichen Umfeld lebt.

Zwar hat die Versorgungsstruktur in der Rehabilitation mit dem Ausbau von wohnortnahen vollstationären, teilstationären und ambulanten Rehabilitationseinrichtungen in den letzten Jahren eine quantitative und qualitative Verbesserung erfahren (Busch \& Diener, 2003). Dennoch ist nach wie vor von einer Unterversorgung 
auszugehen, insbesondere was die ambulanten Sektoren angeht (Sachverständigenrat zur Begutachtung der Entwicklung im Gesundheitswesen, 2005).

Dabei ist die Situation für jüngere Schlaganfallbetroffene noch als günstiger anzusehen als für ältere Patienten, denen eine bedarfsgerechte Rehabilitation bisher nur in den im Bundesgebiet vereinzelt existierenden geriatrischen Modelleinrichtungen zugute kommt (Meier-Baumgartner, 1998, Steinhagen-Thiessen, Hamel, Lüttje, Oster, Plate \& Vogel, 2003). Auch wirken sich die vielen Schnittstellen zwischen verschiedenen Versorgungssektoren, wie z. B. Pflegeleistungen nach SGB XI und ambulante medizinische Leistungen nach SGB V, nachteilig auf die rehabilitative Versorgung von älteren Schlaganfallpatienten aus (Sachverständigenrat für die Konzertierte Aktion im Gesundheitswesen, 2001).

\subsubsection{Implikationen des Krankheitsbildes für die vorliegende Arbeit}

Durch die Darstellung des Krankheitsbildes sollte deutlich gemacht werden, dass ein Schlaganfall eine schwerwiegende Erkrankung ist, die in den meisten Fällen eine tiefgreifende Veränderung im Leben der Betroffenen und ihrer Familienangehörigen bedeutet. Häufig bleiben nach einem Schlaganfall chronische Behinderungen bestehen, die Unterstützung oder Pflege notwendig machen und darüber hinaus eine therapeutische Behandlung bis zum Lebensende erfordern. Oft ist bei Vorhandensein von Risikofaktoren eine zusätzliche Umstellung der Lebensweise notwendig, wobei die Angehörigen gleichermaßen aktiv gefordert sind. Nicht zuletzt leisten die Angehörigen einen wichtigen Beitrag zur emotionalen Bewältigung der Erkrankung.

Die Bedeutung der Familie für den Rehabilitationserfolg wird in der Literatur als sehr hoch eingeschätzt (vgl. Bishop \& Evans, 1995). Glass und Mitarbeiter postulieren, dass ein Misserfolg der Rehabilitation aus einer mangelnden Einbeziehung der Familie resultieren kann (Glass et al., 2004). Oft werden die Beeinträchtigungen, die durch den Schlaganfall bedingt sind, von den Familienmitgliedern missinterpretiert und nicht als Folge der Erkrankung angesehen. Dies resultiert mitunter in aggressiven Gefühlen gegenüber dem Patienten oder aber in einer übertriebenen Fürsorglichkeit. Es mag daher verwundern, dass eine systematische psychologische Begleitung, Schulung und Anleitung der Angehörigen, wie sie in der vorliegenden Arbeit entwickelt und evaluiert 
wurde, in Rehabilitationseinrichtungen nur selten angeboten wird.

\subsection{Begriffsbestimmung: Pflege, Pflegebedürftigkeit, Pflegeperson}

Ein Schlaganfall zieht, wie oben beschrieben, häufig Beeinträchtigungen nach sich, die den Betroffenen abhängig von Hilfe und Unterstützung durch Dritte machen. Nicht immer ist Pflege im engeren Sinne notwendig. Im juristischen Verständnis werden die Begriffe Hilfe- und Pflegebedürftigkeit in Deutschland voneinander abgegrenzt, wobei Leistungen aus der Pflegeversicherung nur bei Anerkennung einer Pflegebedürftigkeit bezogen werden können. Kennzeichnend für das Vorliegen einer Pflegebedürftigkeit ist das Vorhandensein von Einschränkungen im Bereich der Körperpflege, der Ernährung, der Mobilität bzw. Motorik sowie der hauswirtschaftlichen Versorgung. Diese Einschränkungen müssen für mindestens sechs Monate gegeben sein (Besche, 2000). Dagegen liegen bei Hilfebedürftigkeit keine wesentlichen Einschränkungen im körperlichen Bereich vor, eine hauswirtschaftliche Hilfe reicht aus, um eine selbständige Lebensführung zu gewährleisten.

Mit der Einführung der Pflegeversicherung 1994 wurde eine Differenzierung der Pflegebedürftigkeit in drei verschiedene Pflegestufen (§15) vorgenommen. Die Pflegestufen werden wie folgt unterschieden:

1. Pflegebedürftige der Stufe I (erheblich Pflegebedürftige) bedürfen mindestens einmal täglich der Hilfe bei mindestens zwei Verrichtungen im Ablauf des täglichen Lebens (s.o.) und zusätzlich mehrfach in der Woche der Unterstützung bei der hauswirtschaftlichen Versorgung. Der Zeitaufwand muss dabei mindestens 90 Minuten betragen. Hierbei müssen mehr als 45 Minuten auf die Grundpflege entfallen.

2. Pflegebedürftige der Pflegestufe II (Schwerpflegebedürftige) benötigen mindestens dreimal täglich zu verschiedenen Tageszeiten Hilfe bei alltäglichen Verrichtungen und zusätzlich mehrfach in der Woche Unterstützung bei der hauswirtschaftlichen Versorgung. Der Zeitaufwand beträgt mindestens drei 
Stunden, wovon mindestens zwei Stunden auf die Grundpflege entfallen.

3. Pflegebedürftige der Pflegestufe III (Schwerstpflegebedürftige) benötigen rund um die Uhr (auch nachts) Hilfe bei alltäglichen Verrichtungen und zusätzlich mehrfach in der Woche Unterstützung bei der hauswirtschaftlichen Versorgung. Hier müssen mindestens fünf Stunden Zeit pro Tag, vier davon für die Grundpflege, aufgewendet werden.

Im Gesetzestext sind auch Pflegepersonen genau definiert (§ 19 SGB XI):

„Pflegepersonen (...) sind Personen, die nicht erwerbsmäßig einen Pflegebedürftigen im Sinne $§ 14$ in seiner häuslichen Umgebung pflegen. Leistungen zur sozialen Sicherung nach $\S 44$ erhält eine Pflegeperson nur dann, wenn sie eine pflegebedürftige Person wenigstens 14 Stunden wöchentlich pflegt“" (Besche, 2000).

Wenngleich die Einführung der Pflegeversicherung deutliche Verbesserungen für pflegende Angehörige gebracht hat, ist sie nicht ohne Kritik geblieben. Kritisiert wird seitens der Pflegewissenschaft und der pflegerischen Praxis vor allem die enge Begrenzung und Verrichtungsbezogenheit des Begriffs der Pflegebedürftigkeit, die insbesondere bei der Betreuung von Personen mit kognitiven Einschränkungen zu Nachteilen bei der Einstufung führt (Bundesministerium für Familie, Senioren, Frauen und Jugend [BMFSFJ], 2002). Auf der Ebene der Leistungen werden die fehlenden Hilfen für allgemeine Betreuung insbesondere in Form der reinen Beaufsichtigung, aber auch die mangelnd anerkannte zeitliche Intensität der Betreuung beklagt. In einer ersten Überarbeitung des Pflegeversicherungsgesetzes wurde auf diese Kritik reagiert, entsprechend sind in der Fassung von 2003 zusätzliche Betreuungsleistungen eingegangen. Ab 2008 ist eine weitere Veränderung geplant. Diese soll besonders die Situation für kognitiv beeinträchtigte Pflegebedürftige verbessern, die bisher benachteiligt waren. 


\subsection{Demographische Aspekte der familiären Pflege}

Hilfe- und Pflegebedürftigkeit korrelieren in hohem Maße mit dem Alter. So liegt der Anteil der Pflegebedürftigen in der Altersgruppe der 40- bis 64-jährigen durchgängig bei etwa $0.6 \%$. Ab dann nimmt der Anteil der Pflegebedürftigen jedoch exponentiell $\mathrm{zu}$ : Bei den 65 - bis 69 -jährigen beträgt der Anteil $1.7 \%$, steigt auf $6.2 \%$ bei den 75 - bis 79-jährigen und liegt bei den über 85-jährigen bei $26.3 \%$ (Bundesministerium für Familie und Senioren [BMFuS], 1993). Ursache der Pflegebedürftigkeit ist vor allem das Vorhandensein chronischer Mehrfacherkrankungen.

In den nächsten Jahrzehnten ist eine Veränderung der Struktur innerhalb der älteren Bevölkerungsgruppe sowie eine Veränderung der Bevölkerungsverteilung insgesamt zu erwarten (Kohli, 1989). Während der Anteil der über 60-jährigen um 1890 noch bei $5 \%$ lag, beträgt er heute ca. $20 \%$. Bis 2030 soll der Anteil der über 60jährigen um weitere $32 \%$ steigen. Auch der Anteil der Hochaltrigen wächst. Für den Zeitraum von 1986 bis 2030 wird eine Zunahme der über 90-jährigen um 400.000 prognostiziert, was einem Anstieg von $230 \%$ entspricht. Aufgrund des engen Zusammenhangs zwischen Pflegebedürftigkeit und Alter ist also eine Zunahme an Hilfe- und Pflegebedürftigen anzunehmen. Die zwangsläufige Gleichsetzung von hohem Lebensalter mit Pflegebedürftigkeit ist jedoch nicht zulässig, sind doch immerhin $89 \%$ der 70 bis 80 -jährigen und $70 \%$ der 80 bis 90 -jährigen noch in der Lage, allein und ohne größere fremde Hilfe ihren Alltag zu meistern (Lehr, 1991).

Im Gegensatz zur gängigen Meinung, dass Familien sich nicht mehr um ihre alten Angehörigen kümmern, betont Schneekloth (2006), dass nie zuvor so lange und ausdauernd, so viel und aufopfernd gepflegt wurde wie heute. Mehr als $80 \%$ der Pflegebedürftigen werden zu Hause von Angehörigen betreut (Schneekloth \& Leven, 2002). Pflegearbeit ist dabei nach wie vor zum überwiegenden Teil Frauenarbeit und wird meist von einer Hauptpflegeperson geleistet. Ehefrauen sind in $24 \%$ der Fälle die Hauptpflegeperson gegenüber Ehemännern in $13 \%$ der Fälle. Töchter übernehmen zu $26 \%$ die pflegerische Betreuung, Schwiegertöchter in $9 \%$ der Fälle. Söhne und Schwiegersöhne sind nur zu einem äußerst geringen Prozentsatz als Hauptpflegeperson 
tätig (3 \% bzw. 0 \%) (Schneekloth, Potthoff, Piekara \& von Rosenbladt, 1996). Zwar birgt die Übernahme der Pflege durch eine Person den Vorteil der Kontinuität, naturgemäß ist die potentielle Belastung dieser Person jedoch sehr hoch.

In den kommenden Jahren ist davon auszugehen, dass der Prozentsatz der Pflegebedürftigen, die zu Hause betreut werden, sinken wird. Schon im Zeitraum von 1985 bis 1989 ist die Anzahl der Pflegebedürftigen, die von einem Familienmitglied versorgt wurden, um mehr als 200.000 zurückgegangen (Presse- und Informationsamt der Bundesregierung, 1990, zitiert nach KDA, 1992). Rückert (1999) prägte den Begriff der familiären „Fürsorgereserve“ für die Frauen zwischen 45 und 64 Jahren, die den Hauptanteil der Pflegepersonen darstellen. Aufgrund der veränderten Altersstruktur, dem Rückgang der Geburtenzahlen und hohen Scheidungsraten werden immer weniger Frauen aus der „Fürsorgereserve“ Pflege übernehmen können oder wollen, sodass formale Hilfesysteme in Zukunft wichtiger werden (vgl. auch Blinkert \& Klie, 2004).

\subsection{Belastungen durch familiäre Pflege}

Das folgende Kapitel beschäftigt sich mit den Ergebnissen der Forschung zu den Belastungen durch die familiäre Pflege, im Folgenden verkürzend als CaregivingForschung bezeichnet. Zunächst werden die Entwicklungslinien des Forschungsgebietes nachgezeichnet. Es folgt eine Darstellung der wichtigsten empirischen Ergebnisse zu den verschiedenen Aspekten des Belastungserlebens von Angehörigen. Des Weiteren werden Studien zu den positiven Aspekten der Pflege vorgestellt.

Familien von Demenzkranken bilden einen Schwerpunkt in der CaregivingForschung, nur wenige Studien untersuchen dagegen ausschließlich Angehörige von Schlaganfallpatienten. Daher werden im Folgenden auch relevante Studien vorgestellt, die sich mit Pflegenden von Demenzkranken befassen. Beim Vergleich mit der Pflege von Demenzkranken, in den meisten Studien handelt es sich dabei um Menschen mit einer Alzheimer-Demenz, müssen jedoch spezifische Unterschiede berücksichtigt werden: Eine Alzheimer-Demenz tritt typischerweise in einem höheren Lebensalter auf 
als ein Schlaganfall, sodass auch die pflegenden Angehörigen in einem fortgeschritteneren Alter sind. Es ist bei Demenzen keine partielle Remission möglich, beim Schlaganfall können dagegen in vielen Fällen durch eine gezielte Rehabilitation verlorengegangene Fähigkeiten teilweise wiederhergestellt werden. Eine AlzheimerDemenz beginnt schleichend, dagegen tritt ein Schlaganfall plötzlich auf. Außerdem sind bei Demenzkranken, zumindest im frühen und mittleren Stadium, kaum Beeinträchtigungen im motorischen Bereich zu beobachten. Es darf dabei nicht außer Acht gelassen werden, dass einige Schlaganfallbetroffene eine vaskuläre Demenz entwickeln, sodass die pflegenden Angehörigen dann auch mit kontinuierlich abnehmenden kognitiven Fähigkeiten konfrontiert sind.

\subsubsection{Entwicklungslinien der Caregiving-Forschung}

Bis in die siebziger Jahre hinein war eine fachliche und sozialpolitische Wahrnehmung pflegender Angehöriger kaum gegeben. Es lagen nur wenige systematische Erkenntnisse über die Familien chronisch Kranker vor. Die Socialdata-Studie, die Ende der siebziger Jahre in Deutschland durchgeführt wurde, untersuchte zum ersten Mal den Umfang häuslicher Pflege durch Angehörige (Socialdata, 1980). Auch im angloamerikanischen Raum entstand in den siebziger Jahren erstmalig ein breites Forschungsinteresse an dem Thema. Seitdem hat die so genannte Caregiving-Forschung eine kaum noch zu überblickende Vielfalt an Studien hervorgebracht, die sich mit den Auswirkungen der Pflege auf die Angehörigen befassen (im Überblick: Biegel \& Blum, 1990; Gatz, Bengtson \& Blum, 1990; Han \& Haley, 1999; Low, Payne \& Roderick, 1999; Vitaliano, Zhang \& Scanlan, 2003).

Im Fokus der Caregiving-Forschung standen zunächst nur die nachteiligen Aspekte des Pflegeprozesses. Frühe Studien konzentrierten sich vor allem auf die Belastung von Pflegenden allgemein, viele waren rein deskriptiver Natur. Eine theoretische Einbettung wurde häufig nicht vorgenommen. In den Folgejahren zeichnete sich dann eine Differenzierung ab. Als Indikatoren pflegebedingter Belastung wurde nun eine Fülle von Konstrukten und Messinstrumenten eingesetzt.

Zur Erfassung der Beeinträchtigung der psychischen Gesundheit kamen vor 
allem diverse Depressionsinventare zum Einsatz (Haley, Levine, Brown, Berry \& Hughes, 1987; Moritz, Kasl \& Berkman, 1989; Pruchno \& Resch, 1989; Schulz, Tompkins \& Rau, 1988; Stein, Gordon, Hibbard \& Sliwinski, 1992). Weitere Belastungsindikatoren, die untersucht wurden, waren Angst (Dennis, O’Rourke, Lewis, Sharpe \& Warlow, 1998; Kitze, Gründel, Lehmann, Cramon \& Wilz, 2002; Livingston, Brooks \& Bond, 1985), körperliche Gesundheit (Dennis et al., 1998; Franks \& Stephens, 1992; Moritz et al.,1989; Schulz, O’Brien, Bookwala \& Fleissner, 1995; Tyman, 1994), Lebenszufriedenheit (George \& Gwyther, 1986; Wallhagen, 1992; Wright, Lund, Caserta \& Pratt, 1991) und soziale Anpassung (Moritz et al., 1989; Stull, Kosloski \& Kercher, 1994). Neben bereits existierenden Messinstrumenten, die zum überwiegenden Teil aus anderen Forschungsbereichen übernommen wurden, fanden auch Verfahren Verwendung, die eigens für pflegende Angehörige entwickelt wurden. Ein häufig verwendetes $\mathrm{Maß}$ ist die „Burden Scale“, die 1980 von Zarit und Mitarbeitern für Angehörige von dementen Patienten entworfen wurde (Zarit \& Zarit, 1983) und das Ausmaß der Belastung in verschiedenen Bereichen (Gesundheit, Sozialleben, finanzieller Status etc.) misst. Beschränkungen dieses Skalentypus liegen allerdings in der Tatsache, dass die Instrumente nicht variabel für Angehörige von Patienten verschiedener Erkrankungsgruppen einsetzbar sind.

Vor allem von Kramer (1997a) wurde Ende der neunziger Jahre der Mangel an Aufmerksamkeit in Bezug auf die positiven Dimensionen der Pflege kritisiert. Die Autorin postulierte in Übereinstimmung mit der Definition von Gesundheit durch die Weltgesundheitsorganisation (WHO), dass psychische Gesundheit mehr als die Abwesenheit von pathologischen Strukturen umfasst und forderte einen Perspektivenwechsel hin zu positiven Aspekten der Pflege und positiven Indikatoren von Wohlbefinden. Diese Kritik an der Klientifizierung und Psychopathologisierung pflegender Angehöriger wurde von mehreren Forschern aufgegriffen und gab Anlass zu verschiedenen Studien zu dieser Thematik (Beach, 1997; Farran, 1997; Gold, Cohen, Shulman, Zucchero, Andres, Etzezadi \& 1995; Leipold, 2004; im Überblick: Kramer, 1997a).

Neben einer Analyse der Folgen der Pflege für die psychische und körperliche Gesundheit der Angehörigen wurden zunehmend auch diverse kontextuelle Aspekte der Pflegesituation und ihr potentiell moderierender Einfluss auf das Belastungserleben 
beleuchtet. So wurden die Auswirkungen verschiedener personaler und soziodemographischer Aspekte wie Geschlecht, Art der Erkrankung und Wohnsituation untersucht (z. B. Kramer \& Kipnis, 1995; Young \& Kahana, 1989).

Befördert durch die Erkenntnisse zur Belastung von Angehörigen, entstanden parallel zu der eher theoriegeleiteten Forschung praxisbezogene Untersuchungen. Es wurden vielfältige psychoedukative und entlastende Interventionsprogramme entwickelt, die darauf abzielten, die Belastung der Angehörigen zu verringern. Daraus resultierte als weitere wichtige Entwicklungslinie die Evaluationsforschung (Überblicksartikel: Brodaty, 1992; Knight, Lutzky \& Macofsky-Urban, 1993; Schulz et al., 2002; Thompson \& Briggs, 2000; Toseland \& Rossiter, 1989).

Standen die ersten Untersuchungen der Caregiving-Forschung noch in einem „theoriefreien Raum“, wuchs zunehmend das Bemühen um eine Einbettung in theoretische Konzepte (Gottlieb \& Wolfe, 2002). Besonders hervorzuheben ist hier das transaktionale Stressmodell von Lazarus und Mitarbeitern (Lazarus, 1991; Lazarus \& Folkman, 1987), das den meisten Arbeiten im Bereich der Pflegeforschung als theoretische Fundierung dient. Zur Erklärung einzelner Elemente der familiären Pflege wurden auch weitere theoretische Konzepte adaptiert, dazu gehörten u.a. das Konzept der filialen Reife (Bruder, 1988; Blieszner \& Hamon, 1992), die Theorie der sozialen Netzwerke (von Kardorff \& Stark, 1987), die Theorie des sozialen Austausches (Dwyer, Lee \& Jankowski, 1994) sowie die Bindungstheorie (Cicirelli, 1993).

Hinsichtlich der neuesten Entwicklungen in der Angehörigenforschung lassen sich fünf Themenbereiche identifizieren, die in kommenden Jahren zu einem weiteren Erkenntnisgewinn in diesem Forschungsgebiet beitragen dürften.

Zum einen werden in jüngerer Zeit in der Caregiving-Forschung zunehmend die Zusammenhänge zwischen dem Belastungserleben der pflegenden Angehörigen und den zugrunde liegenden Bewältigungsprozessen untersucht (Boeger \& Pickartz, 2001; Lämmler, 2000a, 2000b). Häufig steht dabei ein qualitativer Ansatz im Vordergrund, zum Teil kombiniert mit einem quantitativen Forschungsvorgehen (Wilz, Kalytta \& Küssner, 2005). Dabei lassen sich zwei Forschungsrichtungen unterscheiden. Dem ersten dieser Ansätze liegt eine dynamischere, relationale Vorstellung von Copingprozessen zugrunde (Bridges, 2003; Nolan, Ingram \& Watson, 2002). Die andere 
Forschungsrichtung geht von einem Trait-orientierten Modell aus, welches Copingstile als relativ stabile Persönlichkeitsmerkmale versteht. Dies impliziert, dass über verschiedene Kontexte und Situationen hinweg Bewältigungsformen grundsätzlich generalisiert werden (Haenselt, Danielzik \& Waack, 2004).

Außerdem wird im methodischen Bereich eine Weiterentwicklung von Messinstrumenten vorangetrieben, die auf die Situation pflegender Angehöriger zugeschnitten sind und gute psychometrische Eigenschaften vorweisen (Zank \& Schacke, 2007; Zank, Schacke \& Leipold 2006; Schreiner, Morimoto, Kakuma, Arai \& Zarit, 2006). Diese dienen der differenzierten Beschreibung von Pflegeverläufen und der Evaluation von Interventionen. Neben der Weiterentwicklung standardisierter Messinstrumente ist zu beobachten, dass zunehmend auch qualitative Ansätze integriert werden, womit verstärkt dem prozessualen, dynamischen Charakter der Pflegesituation Rechnung getragen wird (z. B. Gründel, von Cramon \& Wilz, 2003; Lämmler, 1998; McKevitt, Redfern, Mold \& Wolfe, 2004).

Derzeit ist außerdem auf dem Gebiet der Interventionsforschung ein enormer Aufschwung zu beobachten (Coon, Gallagher-Thompson \& Thompson, 2003). Dies hängt eng zusammen mit dem Aufkommen verschiedener innovativer Unterstützungsangebote (z. B. sogenannte Pflegebegleiter, vgl. Dowswell, Lawler \& Young, 2000) und der weiteren Verbreitung und Verfeinerung bewährter Interventionen. Statt globale Veränderungsmaße zu untersuchen, wird zunehmend die Wirksamkeit spezifischer Programmkomponenten in Bezug auf eng umgrenzte Zielvariablen evaluiert, z. B. die Effekte eines Trainings zu kognitiven Umstrukturierungstechniken für Angehörige auf ihre Wahrnehmung von Verhaltensstörungen bei Demenzerkrankten (Mittelman, Roth, Haley \& Zarit, 2004).

Des Weiteren entwickelt sich das Thema „Migranten und Angehörigenpflege“ in Deutschland zu einem wichtigen Forschungsgebiet. Im angelsächsischen Raum ist der Einfluss ethnischer Zugehörigkeit auf bestimmte Aspekte der Angehörigenpflege schon seit geraumer Zeit ein bedeutsames Thema in der Angehörigenforschung (DilworthAnderson, Goodwin \& Williams, 2004). So untersuchten Pinquart und Sörensen (2005) 
in einer Meta-Analyse allein 116 Studien, die sich mit Unterschieden in Stressoren, Ressourcen und psychologischem Wohlbefinden bei verschiedenen ethnischen Gruppen von pflegenden Angehörigen befassten. In Deutschland sind Studien, die die Situation pflegender Angehöriger nicht-deutscher Herkunft untersuchen, noch rar. Mit der wachsenden Gruppe älter werdender Migranten wird dieses Thema in den kommenden Jahren jedoch auch in der deutschsprachigen Pflegeforschung stärker berücksichtigt werden müssen. Erste Ergebnisse eines Projekts („Häusliche Pflege bei Migranten“, Universität Bielefeld) dürften in naher Zukunft veröffentlicht werden.

Schließlich gibt es vielfache Anknüpfungen an die ersten Studien zu den positiven Aspekten und die positive Bewältigung der Pflege, die Ende der neunziger Jahre initiiert wurden. Diese Forschungsrichtung überträgt das theoretische Konzept der Resilienz (Rutter, 1999) auf die Situation der Angehörigenpflege. Resilienz meint die psychologische Widerstandsfähigkeit und das normale Funktionieren trotz vorliegender Risikofaktoren und Belastungen. Dieser Aspekt hat auch in vielen anderen Forschungsgebieten Anwendung gefunden (Carver \& Scheier, 2003; Kim, Schulz \& Carver, 2007; Rieckmann, 2003; Welter-Enderlin \& Hildenbrand, 2006). Eine interessante Studie im Bereich der Angehörigenforschung untersuchte jüngst den Zusammenhang zwischen Resilienz bei pflegenden Angehörigen (geringe Wahrnehmung von Belastung bei gleichzeitig hohen Pflegeanforderungen) mit Institutionalisierung und Mortalität des Pflegebedürftigen (Gaugler, Kane \& Newcomer, 2007). Eine weitere neuere Studie beschäftigt sich mit dem Zusammenhang zwischen Persönlichkeitswachstum und Belastungswahrnehmung von Angehörigen (Leipold, Schacke \& Zank, 2006).

Die Vielfalt der genannten Themen zeigt, dass das Feld der Angehörigenpflege noch längst nicht erschöpfend beforscht ist und auch in Zukunft in den einschlägigen Publikationsorganen Beiträge mit den Schlüsselwörtern „pflegende Angehörige“, „chronische Erkrankungen“ und „Belastung“ zu erwarten sind. 


\subsubsection{Belastung von pflegenden Angehörigen: Empirische Befunde}

Wurden im vorigen Abschnitt die Entwicklungslinien des Forschungsfeldes allgemein beschrieben, sollen nun konkret einige wesentliche Ergebnisse der Forschung dargestellt werden.

In der Caregiving-Forschung entstanden Untersuchungen zu Angehörigen nahezu aller Patientengruppen (z. B. Krebs, orthopädische Erkrankungen, Herzerkrankungen, im Überblick: Biegel, Sales \& Schulz, 1991). Den Schwerpunkt des Forschungsinteresses bilden jedoch Studien mit Familien von zerebral geschädigten Patienten, insbesondere mit Demenzerkrankungen. Dies verwundert nicht, da Angehörige von Patienten mit Hirnschädigungen in der Regel mit erheblich mehr Folgeproblemen konfrontiert sind als Familien von Kranken ohne kognitive Beeinträchtigungen (Davis, 1992).

Die physische Belastung durch die Pflege wird von den pflegenden Angehörigen gegenüber der psychischen meist als weniger gravierend empfunden (Cantor, 1983). Dabei korreliert jedoch nicht das Ausmaß kognitiver Einschränkungen an sich mit dem Belastungserleben bei pflegenden Angehörigen (Fitting, Rabins, Lucas \& Eastham, 1986; Zarit, Reever \& Bach-Peterson, 1980), sondern vielmehr die sozial unangepassten Verhaltensweisen und Wesensänderungen, die häufig mit zerebralen Schädigungen einhergehen (Deimling \& Bass, 1986; Donaldson, Tarrier \& Burns, 1998). Verhaltenssauffälligkeiten wie Antriebsmangel, erhöhte Irritierbarkeit, mangelnde Einsichtsfähigkeit, Aggressivität und Affektlabilität stellen hohe Anforderungen an die kommunikativen Fähigkeiten der Pflegenden. Nicht selten erleben Angehörige Gefühle der Entfremdung, der Trauer und des Verlusts sowie Schuldgefühle gegenüber den Kranken (Kruse, 1984). Beziehungsprobleme und Kommunikationsstörungen treten nicht nur zwischen Pflegendem und Pflegebedürftigem auf: Auch mit anderen Familienmitgliedern sind mitunter Konflikte zu beobachten. Pflegende Angehörige bemängeln oft die fehlende Unterstützung und Anerkennung durch andere Familienangehörige (Pearlin, Mullan, Semple \& Skaff, 1990). Auch kommt es zum Teil aufgrund differierender Ansichten über den „richtigen“ Umgang mit dem Pflegebedürftigen zu unangemessenen Ratschlägen von Seiten der Verwandtschaft. Die hohe zeitliche Belastung durch die Pflege bedeutet eine starke Einschränkung der 
Möglichkeiten zur Freizeitgestaltung. Damit einher geht in vielen Fällen eine Abnahme inner- und außerfamiliärer Kontakte. Dafür ist nicht nur der Mangel an Zeit und Energie der Pflegenden verantwortlich, sondern vielfach auch die Unsicherheit der Umwelt im Umgang mit behinderten Menschen. Dazu kommt die finanzielle Belastung, die sowohl durch die Pflegekosten selbst als auch eventuell durch die Aufgabe der Berufstätigkeit entstehen kann. Als besonders belastend erleben viele Angehörige die wahrgenommene Unveränderbarkeit der Situation (Kruse, 1994).

Dass Angehörige aufgrund der oben beschriebenen Belastungen vielfältige psychische und psychiatrische sowie körperliche Symptome entwickeln können, gilt mittlerweile als gesichert (Low, Payne, Roderick, 1999; Ory, Hoffman, Yee, Tennstedt \& Schulz, 1999; Pinquart \& Sörensen, 2003; Schulz, 2000; Schulz, O’Brien, Bookwala \& Fleissner, 1995). $\mathrm{Zu}$ den am besten untersuchten Indikatoren pflegebedingter Belastung gehören depressive Symptome, die u.a. mit Hilfe der Center of Epidemiological Studies-Depression Scale (CES-D: Radloff, 1977), des Beck Depression Inventary (BDI: Beck, Ward, Mendelsohn, Mock \& Erbaugh, 1961) und der Zung Depression Scale (Zung, 1965) erhoben wurden.

Übereinstimmend konnte nachgewiesen werden, dass pflegende Angehörige hohe Prävalenzraten für depressive Symptome zeigen. Zwar bezieht sich die Mehrzahl der Studien auf Angehörige von Demenzkranken (Cattanach \& Tebes, 1991; Mohide, Pringle, Streiner, Gilbert, Muir \& Tew, 1990; Schulz, O’Brien, Bookwala \& Fleissner, 1995), aber auch Angehörige von Schlaganfallpatienten wurden untersucht. So entwickelten einer finnischen Studie (FINNSTROKE) zufolge ca. $40 \%$ der Angehörigen von Schlaganfallbetroffenen 3 bzw. 12 Monate nach dem Ereignis depressive Symptome (Kotila, Numminen, Waltimo \& Kaste, 1998). Grant und Mitarbeiter kamen zu ähnlichen Ergebnissen (Grant, Weaver, Elliott, Bartolucci \& Newman-Giger, 2004). Schulz, Tompkins und Rau (1988) fanden, dass der Anteil der Angehörigen von Schlaganfallpatienten, die eine depressive Symptomatik entwickeln, etwa 2.5- bis 3.5- mal höher liegt als in der entsprechenden Altersgruppe in der Durchschnittsbevölkerung. Darüber hinaus hatten die Angehörigen, die in der akuten Phase ein hohes Ausmaß an Depression zeigten, ein höheres Risiko, noch ein Jahr nach dem Schlaganfall höhere Depressionswerte vorzuweisen, trotz signifikanter funktionaler Verbesserungen des Patienten. In einer Studie von Lämmler (1998) wiesen 52 \% der 
pflegenden Ehefrauen von Schlaganfallpatienten auffällige Werte in einer Depressionsskala (Geriatric Depression Scale; Yesavage et al., 1983) auf.

Vereinzelt finden sich auch Studien, die eine größere Bandbreite psychiatrischer Symptome untersuchen. Erfasst werden z. B. chronische Erschöpfung, Feindseligkeit und Angst (Anthony-Bergstone, Zarit \& Gatz, 1988; Dura, Stukenberg, \& KiecoltGlaser, 1991; Kinney \& Stephens, 1989; Rabins, Mace \& Lucas, 1982). So fanden Magai, Hartung \& Cohen (1995) bei etwa $10 \%$ der untersuchten Angehörigen eine Angststörung, die nach den DSM-III-R-Kriterien Krankheitswert besitzt. In einer Studie von Lämmler (1998) zeigte sich bei $64 \%$ der Ehefrauen von Schlaganfallpatienten ein erhöhtes Ausmaß an Angstsymptomen (gemessen anhand der Self-rating Anxiety Scale; Zung, 1976).

Die körperliche Gesundheit wurde als Indikator pflegebedingter Belastung bisher sehr unterschiedlich operationalisiert. Am häufigsten kamen subjektive Gesundheitseinschätzungen und Selbstratingskalen, in denen verschiedene körperliche Symptome abgefragt werden, zum Einsatz. Aber auch die Häufigkeit der Arztbesuche, die Menge an eingenommenen Medikamenten sowie der Hormon- und Immunstatus wurden als Maße für die körperliche Gesundheit herangezogen (im Überblick: Pinquart und Sörensen, 2003a, 2007).

In einer Studie von Lämmler (1998) mit Partnerinnen von Schlaganfallpatienten zeigten $73 \%$ der Frauen in einem Inventar körperlicher Beschwerden (Beschwerdenliste; v. Zerssen, 1976) auffällige Werte. Auch Gräßel $(1997,1998)$ kam in seiner Untersuchung mit 1911 Angehörigen von chronisch Kranken mit verschiedenen Krankheitsbildern zu dem Ergebnis, dass Pflegepersonen im Vergleich zur Normalbevölkerung ein signifikant höheres Ausmaß körperlicher und psychosomatischer Beschwerden aufweisen. Ihre Durchschnittswerte waren vergleichbar mit denen von Patienten einer psychosomatisch-psychotherapeutischen Ambulanz. Besonders häufig litten sie an Symptomen körperlicher Erschöpfung sowie Gliederschmerzen (74 \% bzw. $75 \%$ derjenigen, die ein überdurchschnittliches Ausmaß körperlicher Beschwerden aufwiesen) und Magen- und Herzbeschwerden (60 \% bzw. 64 \%). Moritz, Kasl und Ostfeld (1992) untersuchten die Auswirkungen der Pflege eines kognitiv beeinträchtigten Partners auf die körperliche Gesundheit der Pflegenden und fanden einen signifikanten Geschlechterunterschied. Während ein signifikanter 
Zusammenhang zwischen der kognitiven Beeinträchtigung von Ehefrauen und einem erhöhten Blutdruck sowie einer schlechteren subjektiven Gesundheitseinschätzung ihrer pflegenden Männer bestand, fand sich dieser Zusammenhang für pflegende Ehefrauen von kognitiv beeinträchtigten Männern nicht.

Pflege kann auch in physiologischer Hinsicht Auswirkungen auf die Pflegeperson haben: Kiecolt-Glaser und Mitarbeiter (1987) fanden eine deutliche Schwächung der Immunfunktion bei Ehepartnern von Demenzkranken. Auch eine Untersuchung von Döhnert und Mitarbeitern (2001) bestätigte diesen Befund. Den zugrundeliegenden Mechanismus erklärt Döhnert so, dass es aufgrund einer chronisch belastenden Pflegesituation zu einer erhöhten Cortisolausschüttung kommt. Dies hat wiederum eine immunsuppressive Wirkung und führt $\mathrm{zu}$ einer Verminderung der Immunabwehr (Döhnert, Wilz, Adler, Gunzelmann \& Brähler, 2001).

Clipp und George (1990) verwendeten in ihrer Studie als Maß für die körperliche Gesundheit unter anderem die Menge an eingenommenen Medikamenten. Sie konnten nachweisen, dass die Einnahme von psychotropen Substanzen bei pflegenden Angehörigen von Alzheimer-Patienten signifikant höher lag als bei NichtPflegenden. Dabei konnte kein Zusammenhang zwischen der Ausprägung der Krankheit und der Menge der Medikamenteneinnahme festgestellt werden. In Bezug auf die Häufigkeit der Arztbesuche liegen keine eindeutigen Befunde vor (Draper, Poulus, Cole, Poulus \& Ehrlich, 1992; Grafstrom, Fratiglioni, Sandman \& Winblad, 1992). Dies mag mit der Tatsache zusammenhängen, dass Angehörige dazu tendieren, die eigene Gesundheit zu vernachlässigen und bei starker Belastung eher seltener zum Arzt gehen.

Zusammenfassend lässt sich festhalten, dass unabhängig von der Operationalisierung und der verwendeten Konstrukte alle Studien übereinstimmend zu dem Ergebnis kommen, dass pflegende Angehörige im Vergleich $\mathrm{zu}$ anderen gleichaltrigen Personen ohne entsprechende Verantwortung für ein Familienmitglied deutliche Einbußen ihrer psychischen und körperlichen Verfassung erleben. 


\subsubsection{Positive Aspekte bei der Pflege}

Fast die gesamte Caregiving-Forschung bezieht sich auf die Belastungen, die von den Angehörigen bei der Pflege erlebt werden (Nolan, Ryan, Enderby \& Reid, 2002). Einige wenige Studien befassen sich jedoch auch mit den positiven Seiten der Pflege. Cohen, Gold, Shulman und Zucchero (1994) untersuchten den Zusammenhang zwischen positiven Aspekten in der Pflege mit der Entscheidung, einen Pflegebedürftigen zuhause $\mathrm{zu}$ betreuen. Sie stellten fest, dass von den Angehörigen wahrgenommene positive Aspekte ein signifikanter Prädiktor für die Bereitschaft waren, den Pflegebedürftigen zuhause betreuen, nicht aber dafür, ob eine Betreuung tatsächlich zuhause stattfand. Die positiven Aspekte der Pflege waren unter anderem: die Gesellschaft des Pflegebedürftigen zu schätzen, durch die Pflege ein positives Selbstbild zu bekommen und den Pflegebedürftigen zufrieden zu sehen. Jedoch gaben nur 55 \% der Pflegenden spontan positive Aspekte der Pflege an. In anderen Studien $\mathrm{zu}$ den positiven Erfahrungen mit der Familienpflege wurde außerdem von den Teilnehmern geäußert, dass die Pflege für die Angehörigen mit persönlichem Wachstum verbunden ist, sie mehr Nähe zum Pflegebedürftigen entwickelten, sie eine Steigerung ihres Selbstwertgefühls erlebten oder sie ein Gefühl von Wärme und Zusammengehörigkeit empfanden (Chenoweth \& Spencer, 1986; Motenko, 1989). In einem Überblicksartikel zu diesem Themenkomplex (Cohen et al., 1994) zeigte sich, dass die von Studienteilnehmern genannte Anzahl positiver Aspekte in hohem positiven Zusammenhang stand mit einem geringeren Belastungsempfinden, besserer Gesundheit, besserer Beziehungsqualität vor der Erkrankung sowie mehr Zufriedenheit mit der sozialen Unterstützung. In einer qualitativen Studie von Farran, Keane-Hagerty, Salloway, Kupferer \& Wilken (1991) gaben 90 \% der Befragten an, positive Aspekte in der Pflege zu sehen. In einer Studie von Kinney, Stephens, Franks und Norris (1995) berichteten Pflegende sogar von mehr positiven Aspekten als von negativen. Des Weiteren waren Merkmale des Pflegebedürftigen stärkere Prädiktoren von positiven und negativen Aspekten als Charakteristika der Pflegenden.

\section{Zusammenfassung}

Die oben vorgestellten Befunde zur Belastung von pflegenden Angehörigen zeigen, dass die Anforderungen der Pflege einen negativen Einfluss auf die Befindlichkeit der 
Pflegeperson haben können. Die psychische Belastung durch die Pflege wird in den meisten Fällen gegenüber der physischen als gravierender empfunden. Als Folge der Belastung kann es bei pflegenden Angehörigen zu Einschränkungen des psychischen Wohlbefindens bis hin zur Entwicklung von Depressionen und Angstsymptomen sowie psychosomatischen Erkrankungen kommen. Pflegende Angehörige berichten jedoch nicht nur von belastenden Aspekten der Pflege. In Studien zu den positiven Konsequenzen der Pflegetätigkeit nannten Angehörige u. a. persönliches Wachstum, größere Nähe zum Pflegebedürftigen und eine Steigerung des Selbstwertgefühls.

\subsection{Theoretischer Hintergrund der vorliegenden Arbeit}

Die Darstellung der empirischen Befunde zur Belastung von pflegenden Angehörigen im vorhergehenden Kapitel wirft Fragen auf: Wie kommt es, dass manche Angehörige als Folge der Pflegebelastung psychisch oder körperlich erkranken, andere wiederum nicht? Worauf ist die Tatsache zurückzuführen, dass einige Angehörige positive Aspekte in der Pflege sehen, andere nicht? Nicht zuletzt um derartige interindividuelle Unterschiede im Belastungserleben pflegender Angehöriger erklären zu können, wuchs zunehmend das Bemühen um die Entwicklung theoretischer Modelle für den Bereich der Caregiving-Forschung (Harper \& Lund, 1990).

Zur Erklärung einzelner Elemente der familiären Pflege wurden bislang verschiedene Theorien adaptiert, dazu gehören u. a. das Konzept der filialen Reife (Bruder, 1988), die Theorie des sozialen Austausches (Dwyer, Lee \& Jankowski, 1994; Pruchno, 2003; Thiede-Call, Finch, Huck \& Kane, 1999) sowie die Bindungstheorie (Cicirelli, 1991, 1993).

Vor allem bildeten jedoch stresstheoretische Überlegungen und Modelle das theoretische Fundament der Angehörigenforschung. Als bedeutsamste Theorie ist hier das kognitiv-transaktionale-Stressmodell von Lazarus und seinen Kollegen (Lazarus, 1991; Lazarus \& Folkman, 1987) zu nennen. Zahlreiche Studien folgen dem StressCoping-Ansatz (Cohen, 2000; Kramer, 1997a; Lämmler, 1998; Schacke, 2001), das auch als Basis der vorliegenden Arbeit dient und im folgenden Abschnitt erläutert werden soll. 


\subsubsection{Die Kognitiv-transaktionale Stresstheorie}

Zentraler Aspekt des Modells von Lazarus (1991) ist die Postulierung einer wechselseitigen Bedingtheit von Person und Umwelt. Wesentliche Elemente sind dabei Bewertung, Anpassungsversuch, und Neubewertung der situativen Gegebenheiten durch das Individuum.

$\mathrm{Ob}$ eine Stressreaktion entsteht, wird im Wesentlichen dadurch bestimmt, wie eine Person die Umweltgegebenheiten interpretiert. Aus der Fülle der potentiell zur Verfügung stehenden Umweltinformationen filtert das Individuum diejenigen heraus, die Stressrelevanz besitzen könnten. Auf der Basis dieser stressrelevanten Informationen, Reize oder Ereignisse (stressors) werden kognitive Einschätzungen (appraisals) vorgenommen. Bei einer ersten Einschätzung (primary appraisal) wird ein internes Abbild der Situation geschaffen. Es erfolgt eine Bewertung des Ereignisses in Bezug auf drei mögliche Aspekte: Nur dann, wenn eine Situation als Herausforderung, Bedrohung oder Schaden bzw. Verlust interpretiert wird, besitzt sie Stressorqualität. Eine Situation wird als herausfordernd eingeschätzt, wenn sie potentiell mit persönlichem Wachstum, Erfolg oder Gewinn verbunden ist. Bedrohlich erscheint ein Ereignis dann, wenn potentiell ein Schaden oder ein Verlust materieller Güter oder Personen antizipiert wird. Auch potentielle symbolische Beeinträchtigungen können als bedrohlich gewertet werden. Bei Schaden oder Verlust tritt das antizipierte Ereignis tatsächlich ein. Während im Falle der Herausforderung eine Person mit dem Ziel etwas zu gewinnen, aktiv wird, reagiert sie bei Bedrohung, um eine Beeinträchtigung abzuwenden.

Zusätzlich zu der Ereigniseinschätzung, die vorrangig auf der Basis von Umweltinformationen stattfindet, kommt es zu einer Einschätzung der Ressourcen (secondary appraisal) vor dem Hintergrund einer kognitiven Repräsentation der eigenen Person. Dabei werden die eigenen Ressourcen und Fähigkeiten (z. B. soziales Netz, ökonomische Aspekte, Gesundheitsstatus, Problemlösefähigkeiten) mit den Anforderungen der Situation abgeglichen. Wenn die Anforderungen der Umwelt gegenüber den zur Verfügung stehenden Ressourcen größer oder gleich groß erscheinen, erfolgt eine Stressreaktion. Damit hat die subjektive Einschätzung der Situation für das Erleben von Stress eine entscheidende Bedeutung.

Tritt eine Stressreaktion auf, unternimmt das Individuum mit Hilfe adaptiver 
Handlungen oder Gedanken den Versuch, den Stress zu bewältigen. Dabei beeinflusst das Ergebnis der Bewertungsprozesse maßgeblich das Bewältigungsverhalten. Lazarus und Folkman (1984) definieren Bewältigungsverhalten (coping) als "constantly changing cognitive and behavioral efforts to manage specific external and/or internal demands that are appraised as taxing or exceeding the resources of the person" $(1984, \mathrm{~S}$. 141).

Vier Formen der Bewältigung werden nach Folkman und Lazarus (1984) unterschieden:

- Suche nach Information, die in einer Neubewertung der Situation resultieren kann;

- direktes Handeln, das auf eine objektive Veränderung der Umweltbedingungen ausgerichtet ist;

- Unterlassung von Handlungen, die ebenfalls auf eine Veränderung der PersonUmwelt-Beziehungen abzielt;

- intrapsychische Bewältigung, bei der eine Person durch kognitive oder emotionale Prozesse auf die Stresssituation reagiert.

Das Bewältigungsverhalten führt wiederum zu neuen Bewertungsprozessen (reappraisals), die die Einschätzung der auslösenden Situation rückwirkend verändern kann. Es lassen sich neben den Formen auch zwei grundsätzliche Funktionen der Bewältigung unterscheiden, nämlich das problemorientierte und das emotionsorientierte Coping. Bei der problemorientierten Bewältigung wird eine direkte Lösung des Problems angestrebt, indem das Individuum sich aktiv mit dem Stressor auseinandersetzt. Dagegen zielt die emotionsorientierte Bewältigung darauf $a b$, das Ausmaß der erlebten Belastung zu reduzieren (z. B. durch positive Umdeutung, Humor, religiöse Deutung oder Wunschdenken) (Lazarus \& DeLongis, 1983).

Nach Lazarus und Folkman (1984) wirkt sich die Art des Copings einer Person auf ihr psychisches Wohlbefinden, ihre physische Gesundheit und auf ihr Sozialverhalten aus. Grundsätzlich ist eine Bewältigungsstrategie nicht in jeder Situation erfolgreich. Die Funktionalität einer Copingstrategie kann jeweils nur für einen bestimmten Kontext bestimmt werden. In unveränderbaren Situationen können z. B. problemorientierte Bewältigungsstrategien negative Auswirkungen haben, da sie nicht die gewünschte Stressreduktion herbeiführen (Lazarus, 1993a). 
Emotionsorientiertes Copingverhalten wie „Akzeptieren von Belastungen“ kann dagegen z. B. bei einer chronischen Erkrankung eine sehr adaptive Strategie sein. Entsprechend argumentierte Lazarus (1993b), dass es keine Copingstrategie gibt, die nicht grundsätzlich in einem bestimmten Kontext adaptiv sein könnte.

Zusammenfassend zeichnet sich die Stresstheorie von Lazarus gegenüber anderen Modellen dadurch aus, dass der Prozesscharakter des Copings betont wird. Copingprozesse werden nicht als Resultat interaktionistischer, sondern vielmehr transaktionaler Beziehungen zwischen Situations- und Persönlichkeitsvariablen begriffen (zusammenfassend Laux, 1981). Während das interaktionistische Modell im wesentlichen Person und Situation als konstante Größen behandelt und ein unidirektionales Ursache-Wirkungs-Verhältnis annimmt, geht das transaktionale Stressmodell von bidirektionalen Beziehungen aus. Das Copingverhalten hat also entsprechende Rückwirkungen auf die Person sowie auf die Situation. Eine isolierte Betrachtung der Input- und Outputseiten der Stress-Situation wird als wenig sinnvoll erachtet.

Diverse Autoren haben das stresstheoretische Konzept von Lazarus und seinen Mitarbeitern zur Grundlage genommen, um theoretische Modelle zur familiären Pflege zu entwickeln (u. a. Elliott \& Eisdorfer, 1982; Zarit, 1992). Anhand dieser Modelle lassen sich die Faktoren, die für die Situation pflegender Angehöriger relevant sind, leichter systematisieren (Biegel, Sales \& Schulz, 1991). Eine zweckmäßige Übertragung des Stressmodells von Lazarus auf die Situation der familiären Pflege stellt das StressBewältigungsmodell von Kramer (1993b) dar. Dieses dient der vorliegenden Arbeit als theoretische Grundlage und soll im Folgenden vorgestellt werden. 


\subsubsection{Das Stress-Bewältigungsmodell der familiären Pflege von Kramer (1993)}

In diesem Modell wird familiäre Pflege als ein spezifischer Fall der Stressverarbeitung verstanden. Kramer unterscheidet zwischen Merkmalen der Pflegeperson und Stressoren, moderierenden Faktoren wie Copingressourcen und Copingstrategien und den langfristigen Konsequenzen (outcomes) von Stress (vgl. Abbildung 1).

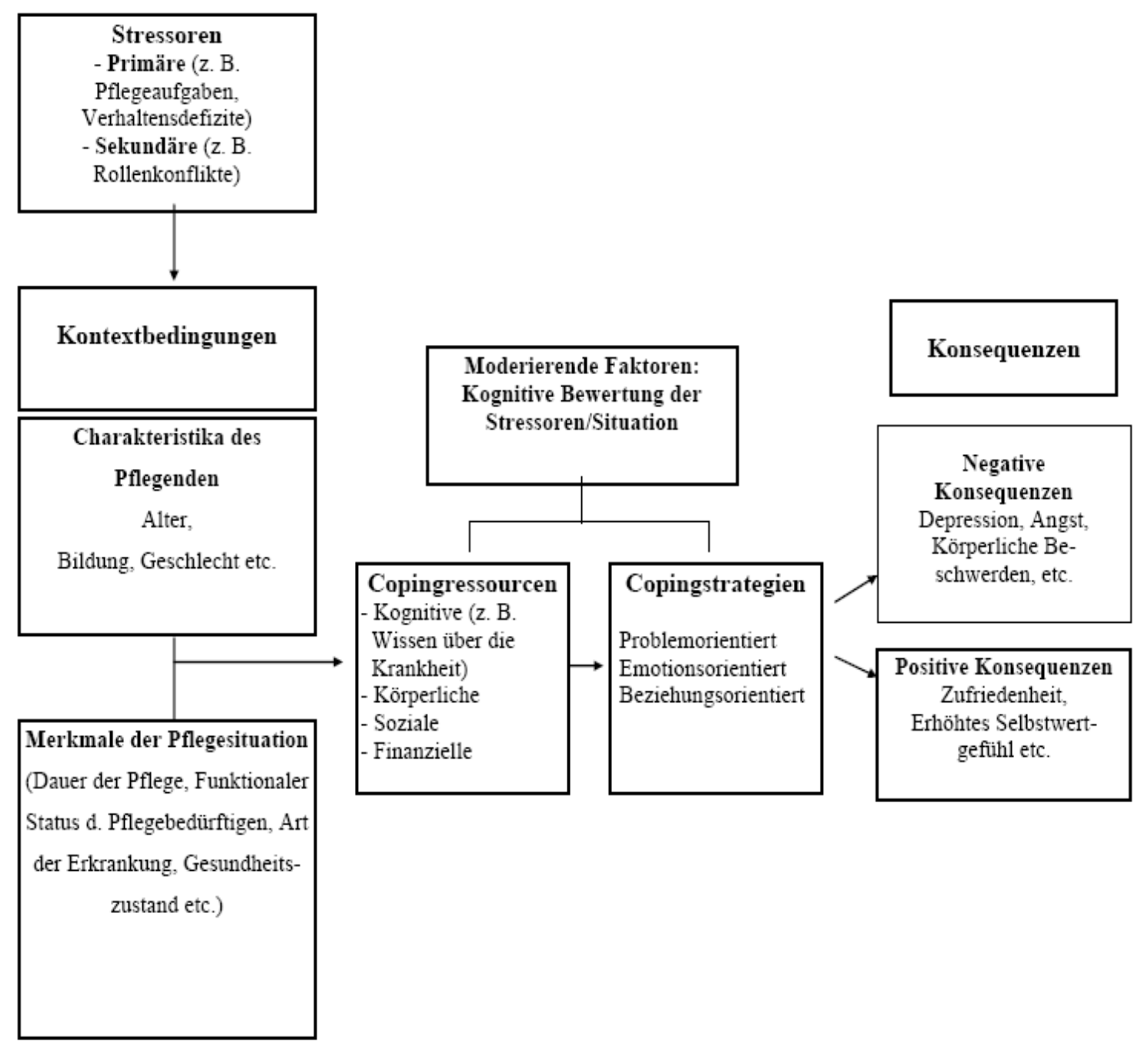

Abbildung 1: Ein theoretischer Rahmen zur Betrachtung von Stressbewältigung in der familiären Pflege (nach Kramer, 1993)

Kramer definiert Stressoren als spezifische Pflegeaufgaben, die sich aus dem funktionalen Status und dem Gesundheitszustand des Pflegebedürftigen ergeben. Dazu 
zählen z. B. die Unterstützung bei den Aktivitäten des täglichen Lebens, der Umgang mit kognitiven Defiziten und mit Wesensveränderungen des Pflegebedürftigen. Die kontextuellen Aspekte der Pflegesituation umfassen soziodemographische Variablen des Pflegenden und des Pflegebedürftigen sowie Merkmale der Beziehung zwischen beiden. Bezug nehmend auf die kognitiv-transaktionale Stresstheorie von Lazarus stellt auch Kramer in ihrem Modell die subjektive Bewertung stressreicher Ereignisse heraus. Moderierende Faktoren sind dabei die zur Verfügung stehenden Copingressourcen und Copingstrategien. Als besonders bedeutsam für die Stressbewältigung in Pflegebeziehungen erachtet Kramer soziale, physische und finanzielle Ressourcen. Die problem- und emotionsorientierten Copingstrategien (Lazarus \& DeLongis, 1983; Folkman \& Lazarus, 1980) erweitert Kramer um Copingstrategien mit interpersonalem Charakter. Diese zielen darauf ab, soziale Beziehungen zu regulieren (z. B. durch die Copingstrategie „einen Kompromiss finden“). Langfristige Konsequenzen der Pflegesituation (outcomes) können nach Kramer sowohl einen negativen Charakter (z. B. Depression, körperliche Symptome) als auch einen positiven Charakter (z. B. Zufriedenheit, erhöhtes Selbstwertgefühl) haben.

Das Stress-Bewältigungsmodell von Kramer ist als gelungene Adaptation der Stresstheorie von Lazarus für die Situation der familiären Pflege zu werten. Allerdings wird durch die graphische Darstellung in ihrem Modell nicht explizit die wechselseitige Beeinflussung der einzelnen Komponenten untereinander herausgestellt und sollte in einer Weiterentwicklung des Modells berücksichtigt werden. Denn wie in den Abschnitten 2.6.2 und 2.6.3 ausführlich dargestellt werden wird, ist davon auszugehen, dass bestimmte Copingstrategien zu einer Veränderung der Reizbedingungen führen können. Auch kann eine Ressource wie soziale Unterstützung unter Umständen selbst zu einem Stressor werden, sodass Rückkopplungen der einzelnen Elemente des Modells aufgenommen werden müssten. 
2.6. Weitere empirische Befunde der Angehörigenforschung vor dem Hintergrund des Stress-Bewältigungsmodell der familiären Pflege nach Kramer

Das Modell von Kramer soll als Rahmenkonzept dienen, um in den folgenden Abschnitten weitere empirische Befunde der Angehörigenforschung darzustellen. Diese basieren in den meisten Fällen zwar nicht explizit auf stresstheoretischen Überlegungen, das oben dargestellte Modell bietet jedoch einen theoretischen Rahmen, in dem die empirischen Befunde sinnvoll geordnet und zusammengefasst werden können. Die längerfristigen Konsequenzen (outcomes) von Stress in Pflegebeziehungen wurden bereits umfassend in den Abschnitten 2.4.2 und 2.4.3 erörtert, sodass im Folgenden nur noch empirische Befunde zu den übrigen Elementen des Modells beschrieben werden.

\subsubsection{Kontextuelle Aspekte der Pflegesituation}

Die strukturellen Faktoren des Pflegekontextes haben entscheidenden Einfluss darauf, wie die Pflegeperson die Situation kognitiv bewertet und welche Bewältigungsstrategien sie anwendet. Dieses wirkt sich wiederum auf das psychische und physische Befinden der Pflegeperson aus. Bislang wurden zahlreiche einzelne Variablen des Pflegekontextes sowie Gruppen von Variablen untersucht. Dazu zählen u. a. die Art der Erkrankung des Patienten, der Grad der Pflegebedürftigkeit, die Pflegedauer, das Verwandtschaftsverhältnis, das Wohnarrangement sowie das Geschlecht des Angehörigen. Die Ergebnisse sind jedoch vor dem Hintergrund einer methodischen Einschränkung zu sehen: Einige Faktoren, z. B. Verwandtschaftsverhältnis und Wohnsituation, sind miteinander konfundiert und lassen daher eine isolierte Betrachtung nur im Rahmen sehr großer Stichproben und bei Anwendung spezieller statistischer Prozeduren zu.

Eine Langzeitstudie (Schulz, Tompkins \& Rau, 1988), in der verschiedene kontextuelle Variablen wie Alter und Einkommen analysiert wurden, untersuchte Angehörige von Schlaganfallbetroffenen. Der Zusammenhang der kontextuellen Variablen mit der Entwicklung depressiver Symptome bei den pflegenden Angehörigen veränderte sich stark von der akuten zur chronischen Phase der Erkrankung. 
Unmittelbar nach dem Akutereignis gab es keinen Zusammenhang zwischen den soziodemographischen Faktoren wie Alter und Einkommen und dem Ausmaß depressiver Symptome bei den Angehörigen. Acht Monate nach dem Schlaganfall korrelierten diese Variablen allerdings signifikant mit Depression bei den Angehörigen. Ältere Pflegende mit gutem ökonomischem Status und guter Gesundheit zeigten die geringsten depressiven Symptome. Die Autoren kamen zu dem Schluss, dass typische soziodemographische Variablen (Alter, Einkommen etc.) besser das Wohlbefinden der Pflegenden im zeitlichen Verlauf vorhersagen als Schlaganfall-bezogene Variablen (Ausprägung der Beeinträchtigungen etc.).

\section{Verwandtschaftsverhältnis}

Inwieweit das Verwandtschaftsverhältnis für das Belastungserleben eine Rolle spielt, konnte bislang nicht eindeutig beantwortet werden. In Studien zur Angehörigenbelastung werden vorwiegend Ehefrauen oder Töchter von chronisch Kranken befragt, was damit zusammenhängt, dass diese am häufigsten in die häusliche Pflege eingebunden sind. In der Altersgruppe der 65- bis 79-jährigen Pflegebedürftigen sind die Hauptpflegepersonen zu $61 \%$ die Ehepartnerinnen. Erst im höheren Alter erfolgt ein Generationensprung und die Pflege wird vorwiegend von Kindern, insbesondere den Töchtern, übernommen (Franke, 2000).

Einer Studie von Magai, Hartung und Cohen (1995) zufolge leiden Ehefrauen häufiger unter psychologischem Distress und persönlicher Belastung, während pflegende Töchter eher Rollenkonflikte und interpersonale Belastungen erfahren. George und Gwyther (1986) und Gilhooly (1984) fanden in ihren Untersuchungen, dass Ehepartner im Gegensatz zu Kindern ein schlechteres psychisches Wohlbefinden aufweisen. Dieses Ergebnis lässt sich möglicherweise dadurch erklären, dass Ehepartner älter sind und damit durch die Pflege körperlich und emotional stärker beansprucht werden. Dagegen fanden Zarit, Reever und Bach-Peterson (1980) und Quayhagen und Quayhagen (1988) keine Unterschiede in der Belastung zwischen pflegenden Ehepartnern und Kindern. Eine mögliche Erklärung dafür ist, dass Kinder häufig verschiedene Rollen ausfüllen müssen und dadurch mehrfachen Belastungen ausgesetzt sind - in der Literatur wird zutreffend von der "Sandwich-Generation“ gesprochen (Brody, 1981). 
Eine Arbeit von Peters-Davis, Moss und Pruchno (1999) verglich die Erfahrungen von Schwiegertöchtern und leiblichen Töchtern bei der Pflege hilfsbedürftiger (Schwieger-) Eltern. Im Gegensatz zu den Erwartungen der Autoren erlebten die leiblichen Kinder und Schwiegerkinder ähnliche Belastungen bei der Pflege. Die Ergebnisse implizieren, dass es ist nicht allein die Art der Beziehung ist, die einen Einfluss auf die Belastung durch die Pflege hat, sondern auch die Qualität der Beziehung.

\section{Qualität der Beziehung zwischen Pflegebedürftigem und Pflegenden}

Da der Pflegesituation in den meisten Fällen eine lange Beziehungsgeschichte vorausgeht, liegt es auf der Hand, dass die (frühere) Beziehungsqualität zwischen Pflegebedürftigem und Pflegenden für das Belastungserleben der Pflegeperson eine große Rolle spielt. Allerdings existieren nur wenige Studien, die sich mit dieser Thematik beschäftigen, was zweifellos auch auf die Komplexität der Fragestellung und die damit verbundene schwierige Operationalisierung zurückzuführen ist.

Der allgemeinen Erwartung entsprechend, fanden einige Autoren, dass eine schlechtere Beziehungsqualität vor der Erkrankung in einer höheren Ausprägung der Belastung der Pflegenden resultiert (Williamson \& Schulz, 1990; Zarit, Todd \& Zarit, 1986). Insbesondere erhöhen nicht bearbeitete Beziehungskonflikte die Belastung für pflegende Ehepartner (Kramer, 1993a). Aber nicht nur eine schlechte Beziehung kann das Belastungserleben beim Pflegenden vergrößern, sondern auch eine sehr gute Beziehung. Dies begründen Biegel, Sales und Schulz (1991) damit, dass die Auseinandersetzung mit Verlustängsten für pflegende Angehörige in sehr harmonischen Beziehungen besonders belastend sein kann.

Adler, Gunzelmann, Machold, Schumacher und Wilz (1996) gingen der Frage nach, inwieweit das Belastungserleben mit qualitativen Merkmalen der emotionalen Beziehung zwischen pflegender und erkrankter Person zusammenhängt. Als bedeutender Faktor erwies sich die Autonomie bzw. Abhängigkeit des Pflegenden vom Kranken. Sehr abhängige Angehörige stellten eigene Bedürfnisse ständig zurück, entfernten sich nur mit Schuldgefühlen vom Kranken, fühlten sich unentbehrlich in der Pflege und lehnten Hilfsangebote ab. Dies resultierte, unabhängig von der objektiven pflegerischen Belastung, in einem höheren Ausmaß an sozialer Isolierung, depressiver 
Symptomatik, gesundheitlichen Problemen und Ängstlichkeit. Motenko (1989) stellte in einer Studie mit 50 Ehefrauen von Alzheimerpatienten fest, dass die wahrgenommene Kontinuität in der Beziehung ein wichtiger Einflussfaktor für das Wohlbefinden der Frauen war. So empfanden Frauen, die sich sowohl vor als auch nach Beginn der Erkrankung ihrem Mann nahe fühlten bzw. nicht nahe fühlten, ihre Rolle als zufrieden stellender, als Frauen, bei denen sich das Erleben von Nähe durch die Erkrankung stark gewandelt hatte. Darüber hinaus waren die pflegenden Angehörigen zufriedener, die als Motivation für die Übernahme der Pflege Reziprozität oder Liebe angaben, im Gegensatz zu denen, die aus Pflichtgefühl pflegten.

Grundsätzlich sind Untersuchungen $\mathrm{zu}$ diesem Themenkomplex methodisch einigen Herausforderungen gegenübergestellt. Das Konzept der Beziehungsqualität ist sehr komplex und retrospektiv schwer zu erfassen. Eine qualitative Herangehensweise wird der Thematik zweifelsohne eher gerecht als eine rein quantitativ orientierte.

\section{Art der Erkrankung}

Viele Studien konnten nachweisen, dass die Art der Erkrankung des Pflegebedürftigen entscheidende Bedeutung für das Belastungserleben der Angehörigen hat (Pickett, Altmaier \& Paulsen, 2007; Thommessen, Aarsland, Brackhus, Oksengaard, Engedal \& Laake, 2002). Die Pflege eines Menschen mit kognitiven Defiziten geht mit einer größeren Belastung für die Angehörigen einher als die Pflege eines rein körperlich Erkrankten. (Biegel et al., 1991; Gräßel, 1997; Leipold, Schacke \& Zank, 2005; Pearlin et al., 1996). So zeigten verschiedene Autoren, dass Angehörige, die kognitiv Beeinträchtigte pflegen, höhere Werte in verschiedenen Belastungsindikatoren (Depressions- und Angstskalen, körperliche Beschwerden, subjektive Belastung) aufweisen als Angehörige von rein körperlich Erkrankten oder Nicht-Pflegende (Clipp \& George, 1993; Houlihan, 1987; Sistler, 1989). Es sind jedoch nicht so sehr die kognitiven Defizite selbst, die mit Stresserleben assoziiert sind, sondern vielmehr die Verhaltensstörungen, die aus den kognitiven Beeinträchtigungen resultieren (Bédard, Molloy, Pedlar, Lever \& Stones, 1997; Coen, Swanwick, O’Boyle \& Coakley, 1997; Donaldson, Tarrier \& Burns, 1997; Johannsen, 1994). Während Angehörige von rein körperlich Erkrankten vor allem pflegerische Aufgaben im engeren Sinne leisten müssen, die in erster Linie physisch belastend sind, kommt auf Personen, die einen 
kognitiv Beeinträchtigten versorgen, eine Fülle von zusätzlichen Aufgaben hinzu. Dazu gehören die Strukturierung des Alltags, der Umgang mit Verhaltensauffälligkeiten sowie die Anleitung zu Tätigkeiten. Dabei müssen Angehörige ein Mehrfaches an emotionaler Energie aufbringen. Scharlach (1987) konnte darüber hinaus nachweisen, dass Angehörige, die einen Patienten mit kognitiven Beeinträchtigungen pflegen, etwa eineinhalb Mal so viel Zeit mit der Pflege zubringen, als Angehörige, die einen körperlich Kranken versorgen. Erstere fühlten sich außerdem stärker gefordert und erlebten mehr Stress. Auch der Umgang mit sprachgestörten Pflegebedürftigen wird von den pflegenden Angehörigen als sehr belastend empfunden. Ehepartner von aphasischen Patienten zeigen generell ausgeprägtere Anpassungsprobleme als Partner nicht sprachgestörter Patienten (Draper, Bowring, Thompson, Van Heyst, Conroy \& Thompson, 2007). So gaben Angehörige aphasischer Patienten deutlich weniger soziale Aktivitäten an als Angehörige nichtaphasischer Schlaganfallbetroffener (Denman, 1998).

\section{Grad der Pflegebedürftigkeit}

Studien, die den Grad der Pflegebedürftigkeit als Prädiktor für die Belastung des Pflegenden untersuchen, kommen zu uneinheitlichen Ergebnissen. In einigen Studien zeigte sich, dass mit einer Zunahme der Pflegetätigkeiten auch das Belastungserleben der pflegenden Angehörigen ansteigt (Barber, 1988; Cicerelli, 1993; Scharlach, 1987). Andere Autoren wiederum kamen zu dem Schluss, dass kein Zusammenhang zwischen dem Ausmaß der Pflegebedürftigkeit und dem Grad der Belastung der Angehörigen besteht (Gallant \& Connell, 1997; Hooker, Monahan, Shifren \& Hutchinson, 1992; Kramer, 1993a).

Die Uneinheitlichkeit der Ergebnisse liegt möglicherweise darin begründet, dass unterschiedliche Messinstrumente verwendet wurden. In Studien, in denen auch supervisorische Tätigkeiten bei den Pflegehandlungen berücksichtigt werden, zeigen sich generell eher Zusammenhänge mit dem Belastungsgrad der Angehörigen als in Studien, die das Ausmaß der Pflegebedürftigkeit über reine Pflegetätigkeiten definieren. Wie oben schon erwähnt, steht der Grad der Pflegebedürftigkeit eng in Zusammenhang mit der Art der Erkrankung. 


\section{Pflegedauer}

Auch in Bezug auf den Zusammenhang zwischen der Pflegedauer und der Belastung der Angehörigen finden sich in der Literatur keine einheitlichen Befunde. Generell lassen sich zwei verschiedene Konzepte unterscheiden: Die "Wear-and-tear”-Hypothese und das Adaptionsmodell. Die "Wear-and-tear"-Hypothese geht davon aus, dass sich mit der Zeit die psychische Belastung durch die Pflege kumulativ verstärkt (Haley \& Pardo, 1989; MacNamara, Gummow, Goka \& Gregg, 1990; Townsend, Noelker, Deimling \& Bass, 1989). Für diese These sprechen auch Befunde von Bodnar und Kiecolt-Glaser (1994): Sogar lange Zeit nach dem Tod des Pflegebedürftigen zeigen ehemalige Pflegende erhöhte Werte in Depressionsskalen. Dies begründen die Autoren damit, dass durch die kontinuierlichen Belastungen positive Ressourcen nicht mehr ausgeschöpft werden können. So kommt es über die Jahre der Pflegetätigkeit oft $\mathrm{zu}$ massiven Einschränkungen in der Freizeit und in den sozialen Beziehungen, die nach dem Tod des Kranken schwer wieder zu aktivieren sind.

Im Gegensatz dazu postulieren Vertreter der Adaptionshypothese, dass sich pflegende Angehörige im Laufe der Zeit an die belastende Situation anpassen und effektivere Bewältigungsstrategien anwenden (Pearlin, Lieberman, Menaghan \& Mullan, 1981). Diese These unterstützen u. a. Studien von George und Gwyther (1986), Haley, Levine, Brown und Bartolucci (1987) und Kramer (1993b), die keine signifikanten Zusammenhänge zwischen der Pflegedauer und der emotionalen Befindlichkeit der Pflegenden fanden. Gilhooly (1984) fand sogar, dass sich das psychische Wohlbefinden pflegender Angehörigen von Demenzkranken besserte, je länger die Pflege andauerte. Die Ergebnisse von Given, Given, Stommel und Azzouz (1999) stützen ebenfalls das Adaptationsmodell. Sie konnten nachweisen, dass Angehörige von frisch aus dem Krankenhaus entlassenen Pflegebedürftigen signifikant höhere Depressionswerte zeigten als Angehörige, die schon länger einen chronisch Kranken versorgten.

Die uneinheitlichen Ergebnisse der Forschung in Bezug auf die Auswirkungen der Pflegedauer überraschen nicht, wenn man in Betracht zieht, dass die Pflegedauer eng verwoben ist mit der Art der Erkrankung und dem Krankheitsverlauf und daher ein konfundierender Effekt zu erwarten ist. 


\section{Wohnsituation}

In Bezug auf die Frage, ob ein gemeinsamer oder ein getrennter Haushalt zu einer höheren Belastung der pflegenden Angehörigen führt, kommt Parker (1997) zu dem Schluß, dass ein gemeinsames Wohnarrangement eine höhere Belastung für die Pflegeperson darstellt als ein getrenntes. Befunde von Cohen und Eisdorfer (1988) stützen ebenfalls diese Aussage: 55 \% der Angehörigen, die einen Demenzkranken im eigenen Haushalt betreuen, erfüllten die DSM-III-Kriterien für eine unipolare Depression bzw. für eine Anpassungsstörung mit depressiven Symptomen. Dagegen erfüllte keiner der Pflegenden, die getrennt vom Kranken lebten, diese Kriterien. Deimling, Bass, Townsend \& Noelker (1989) weisen darauf hin, dass der Schwerpunkt der potentiellen Konflikte zwischen Pflegebedürftigem und Pflegeperson je nach Wohnarrangement unterschiedlich gelagert ist. Angehörige, die mit dem Pflegebedürftigen in einem Haushalt zusammenwohnen, fühlen sich in Bezug auf den Alltag und die Ausübung sozialer Aktivitäten eher eingeschränkt. Dagegen ergibt sich bei Pflegepersonen, die nicht mit dem Kranken zusammenleben, eher Konfliktpotential aufgrund des Spannungsfeldes zwischen den Anforderungen der Pflege und eigenen familiären Pflichten. So verwundert es nicht, dass in einer Studie von Harper und Lund (1990) die am stärksten belastete Gruppe pflegende Töchter darstellen, die mit einem

pflegebedürftigen Elternteil zusammenleben. Allerdings könnte hier ein konfundierender Faktor eine Rolle spielen: Pflegebedürftige, die mit ihren Kindern zusammenwohnen, sind meist älter und stärker beeinträchtigt und erfordern damit mehr Einsatz als diejenigen, die noch in ihrem eigenen Haushalt gepflegt werden (Deimling et al., 1989). Generell gilt auch für den Aspekt Wohnsituation, dass konfundierende Effekte zu erwarten sind, da der Verwandtschaftsgrad häufig das Wohnarrangement beeinflusst.

\section{Geschlecht}

Die Mehrzahl der Studien, die sich mit Geschlechterdifferenzen bei der Bewältigung der Pflege befassen, zeigten, dass sich pflegende (Ehe-)Männer weniger subjektiv belastet fühlen als pflegende Frauen und seltener depressive Störungen und unspezifische körperliche Beschwerden aufweisen (Barusch \& Spaid, 1989; Bédard, Kuzik, Chambers, Molloy, Dubois \& Lever, 2005; Fitting, Rabins, Lucas \& Eastham, 1986; 
Rose-Rego, Strauss \& Smyth, 1998; Zarit, Todd \& Zarit, 1986; vgl. auch die Metaanalyse von Miller und Cafasso, 1992). Allerdings finden sich auch Studien, die keine Unterschiede zwischen männlichen und weiblichen Pflegepersonen nachweisen konnten (Gräßel, 1997; Quayhagen \& Quayhagen, 1988; Rößler, 1995).

In einer Studie von Moritz, Kasl und Berkman (1989) dagegen zeigte sich bei pflegenden Ehemännern von kognitiv Beeinträchtigten ein positiver Zusammenhang zwischen dem Grad der kognitiven Beeinträchtigung der Frauen und der depressiven Verstimmung der Ehemänner. Dieser Zusammenhang konnte bei den pflegenden Ehefrauen nicht nachgewiesen werden. Die Autoren führen als Begründung Studien zur psychischen Gesundheit und Familienstatus an, in denen nachgewiesen wurde, dass Männer mehr von der Ehe profitieren als Frauen. Ein „Verlust“ des Ehepartners durch schwerwiegende kognitive Beeinträchtigungen dürfte Männer demnach stärker treffen als Frauen.

Miller (1987) kam in einer explorativen Studie zu anderen Ergebnissen. Sie verglich Ehefrauen und Ehemänner, die ihre demenzkranken Partner pflegten und stellte fest, dass Ehemänner leichter die Kontrolle über die Pflegesituation behielten. Als Erklärung führte die Autorin an, dass verinnerlichte Geschlechtsrollen in hohem Maße den Umgang mit der Pflegesituation beeinflussen. Männern falle es aufgrund ihrer Sozialisation leichter als Frauen, Grenzen zu setzen und eigene Interessen auszuleben. Außerdem legten Männer und Frauen unterschiedliche Strategien zur Bewältigung der Pflege an den Tag. Männliche Pflegepersonen neigen eher $\mathrm{zu}$ aktivem CopingVerhalten, während weibliche eher $\mathrm{zu}$ einem passiveren, emotionsregulierenden Bewältigungsstil tendieren (Borden \& Berlin, 1990). Weibliche Pflegende sind im Unterschied zu männlichen Pflegepersonen weniger zufrieden mit ihren sozialen Kontakten und erleben größeren finanziellen und physischen Stress (Schulz \& Williamson, 1991). Ein weiteres Erklärungsmodell liefert Enright (1991): Frauen erhalten bei der Pflege eines demenzkranken Familienmitgliedes weniger Unterstützung vom sozialen Umfeld als Männer, die meist durch andere (weibliche) Angehörige entlastet werden. Zudem seien Männer eher in der Lage Aufgaben zu delegieren. Einen zusätzlichen Gesichtspunkt führen Rose-Rego, Strauss und Smyth (1998) an. Möglicherweise nehmen Frauen eher Beschwerden wahr und thematisieren sie leichter in Befragungen als Männer. Bédard und Mitarbeiter argumentieren schließlich, dass es 
eine höhere Prävalenz von Verhaltensstörungen und Aggressivität bei Männern mit kognitiven Beeinträchtigungen gibt. Da die subjektive Belastung in hohem Maße mit dem Ausmaß an Verhaltensstörungen zusammenhängt, wäre somit auch zu erklären, warum Frauen sich tendenziell stärker belastet fühlen (Bédard, Kuzik, Chambers, Molloy, Dubois \& Lever, 2005).

\section{Alter}

In der Caregiving-Literatur finden sich keine einheitlichen Aussagen hinsichtlich des Einflusses des Alters der Pflegeperson auf das Belastungserleben. Dies lässt sich vermutlich durch den Umstand erklären, dass Alter eher eine Indikatorvariable darstellt und mit anderen Faktoren wie physischen Ressourcen oder konkurrierenden Rollenverpflichtungen verwoben ist (Horowitz, 1985b). Somit sind die Ergebnisse zu dieser Fragestellung stark von der Zusammensetzung der Stichprobe abhängig. In einigen Studien nimmt die Belastung und psychische Beeinträchtigung der Pflegeperson mit zunehmendem Alter ab (Clipp \& George, 1993; Kramer \& Kippis, 1995; Spaid \& Barusch, 1994). Intrieri und Rapp (1994) hingegen fanden eine Zunahme der Belastung durch die Pflege mit ansteigendem Alter (vgl. auch Biegel, Sales \& Schulz, 1991, S. 205). Andere Studien wiederum konnten keinen Zusammenhang zwischen Alter und Belastung feststellen (Harper \& Lund, 1990; Kosberg, Cairl \& Keller, 1990).

\section{Zusammenfassung}

Die obigen Ausführungen zeigen, dass der Pflegekontext einen entscheidenden Beitrag zur Ausbildung pflegebedingter Belastungsreaktionen der Angehörigen leistet. Obwohl viele der Befunde uneinheitlich sind, können einige Erkenntnisse als gesichert bezeichnet werden. So hat die Art der Erkrankung wesentlichen Einfluss auf das Belastungserleben der Pflegenden. Die Pflege eines Patienten mit kognitiven Beeinträchtigungen führt $\mathrm{zu}$ einem besonders hohen Belastungsempfinden bei den Angehörigen. In Bezug auf andere Kontextmerkmale wie Geschlecht des Pflegenden, Verwandtschaftsverhältnis und Wohnsituation sind die empirischen Belege weitaus widersprüchlicher. Die Uneinheitlichkeit der Befunde spiegelt jedoch die Komplexität der Zusammenhänge wider. Daraus ergibt sich, dass eine isolierte Betrachtung einzelner Kontextmerkmale wenig zweckmäßig ist, da sich nur durch die Kombination diverser 
Kontextmerkmale ein angemessenes Abbild der Realität schaffen lässt.

\subsubsection{Copingressourcen}

Wie unter 2.5.1 schon beschrieben, setzt eine effektive Stressbewältigung voraus, dass ausreichend Copingressourcen vorhanden sind, um die Stressoren reduzieren zu können oder um eine Adaptation des Individuums an die Stressoren herbeizuführen. Im StressBewältigungsmodell der familiären Pflege führt Kramer (1993) physische, soziale und finanzielle Ressourcen als besonders bedeutsam für die Pflegesituation an. Kahana, Kahana und Kinney (1990) berücksichtigen in ihrem Stressbewältigungsmodell zusätzlich noch internale Copingressourcen wie kognitive Fähigkeiten (z. B. Wissen über ein Krankheitsbild), Wertvorstellungen und Aspekte des Selbstbildes. Diese beeinflussen entscheidend die Nutzung bestimmter Copingstrategien. Da es innerhalb der Caregiving-Forschung jedoch kaum empirische Befunde $\mathrm{zu}$ den internalen Copingressourcen gibt, konzentrieren sich die folgenden Abschnitte auf Studien zu finanziellen und sozialen Ressourcen.

\section{Finanzielle Ressourcen}

Die Auswirkungen der ökonomischen Situation auf die Belastung pflegender Angehöriger sind in nur wenigen Studien untersucht worden. In den meisten Fällen ließ sich kein Zusammenhang zwischen der Einkommenshöhe und dem Ausmaß der Belastung nachweisen (Kosberg, Cairl \& Keller, 1990; Thomson, Futterman, GallagherThomson, Rose \& Lovett, 1993). Stone, Cafferata und Sangle (1987) wiesen jedoch darauf hin, dass finanzielle Ressourcen als moderierende Variable einen Einfluss auf die Belastung haben, da pflegende Angehörige eher soziale Dienstleistungen wie Tagespflege in Anspruch nehmen, wenn ausreichend finanzielle Mittel zur Verfügung stehen.

\section{Soziale Unterstïtzung}

Die Forschung zum Thema soziale Unterstützung als Ressource erfreut sich großer Beliebtheit seit das Konzept erstmals in den frühen 1970er Jahren untersucht wurde und gilt als zentraler Aspekt in der Streß- und Coping-Literatur (Hobfoll, 1988; Lazarus, 
1991). Forscher haben sich sowohl um die quantitativen wie auch qualitativen Dimensionen der sozialen Unterstützung bemüht (Bruhn \& Phillips, 1984).

In der Literatur zum Thema soziale Unterstützung ist insgesamt eine große Begriffsvielfalt zu konstatieren. Neben dem Konzept der sozialen Unterstützung werden auch die Begriffe soziales Netzwerk oder soziale Integration verwendet. Diese müssen jedoch klar voneinander abgegrenzt werden (Dunkel-Schetter \& Bennett, 1990). Während die soziale Integration das Vorhandensein von sozialen Beziehungen meint, bezieht sich das soziale Netzwerk auf die Struktur der Beziehungen. Dagegen beschreibt der Begriff soziale Unterstützung die problemlösende und/oder leidreduzierende Funktion der sozialen Beziehungen.

Mittlerweile gilt als gesichert, dass die prinzipielle Verfügbarkeit von sozialer Unterstützung einen stressreduzierenden Effekt hat. Durch Stress hervorgerufene schädliche Verhaltensweisen oder biologische Reaktionen, die negative physiologische und psychische Konsequenzen für die Gesundheit haben, können durch soziale Unterstützung verhindert oder eingeschränkt werden (Chwalisz, 1992). Der positive Effekt sozialer Unterstützung ist bislang anhand verschiedener Variablen wie psychische Gesundheit, Lebenszufriedenheit sowie Krankheitsrisiko nachgewiesen worden (u. a. Antonucci \& Jackson, 1987; Forster \& Stoller, 1992; Sarason \& Sarason, 1985). Allerdings darf nicht vernachlässigt werden, dass Unterstützung durch das soziale Netzwerk nicht nur als angenehm und entlastend, sondern mitunter auch als kontrollierend und unangenehm empfunden werden kann (Laireiter \& Lettner, 1993).

Grundsätzlich gilt es, die wahrgenommene (erwartete) und die tatsächlich erhaltene Unterstützung voneinander $\mathrm{zu}$ unterscheiden. Die wahrgenommene Unterstützung bezieht sich auf die Überzeugung einer Person, im Bedarfsfall Hilfe aus dem sozialen Netz zu erhalten. Schwarzer (1993) nennt drei verschiedene inhaltliche Aspekte der sozialen Unterstützung: die emotionale Unterstützung (Anteilnahme, Trost), die instrumentelle Unterstützung (Übernahme von Tätigkeiten) sowie die informationsbezogene Unterstützung (Vermittlung von Informationen und Problemlösungsvorschlägen). 
Wie oben schon erwähnt, ist die subjektive Bewertung der sozialen Unterstützung durch die Hilfeempfänger ein bedeutender Faktor. So konnte nachgewiesen werden, dass die subjektive Einschätzung der sozialen Unterstützung einen größeren Einfluss auf das Wohlbefinden hat als die objektiv messbare Menge an Unterstützung oder sozialem Austausch (Jackson \& Antonucci, 1992). Auch Laireiter und Lettner (1993) widmen sich der subjektiven Bewertung sozialer Unterstützung und konstatieren in ihrer Übersicht zu den negativen Aspekten von Hilfe, dass diese sogar einen stärkeren Einfluss auf die Gesundheit besitzen als die positiven Faktoren. Sie nennen unter anderem folgende Gesichtspunkte in Bezug auf negative Konsequenzen sozialer Unterstützung (vgl. auch Coates, Renzaglia \& Embree, 1983):

Ist die Beziehung zwischen Hilfeempfangenden und -gebenden von einem Machtgefälle gekennzeichnet, kann soziale Unterstützung als Kontrolle empfunden werden. Auch können aufgrund der Unterstützung negative Gefühle wie Selbstwertprobleme, Gefühle der Scham, Schuld oder Verpflichtung entstehen. Wenn Unterstützung erwartet, aber nicht gewährleistet wird, kann dies außerdem vom Hilfesuchenden als Kränkung erlebt werden. Übermäßige Hilfe wiederum kann dazu führen, dass sich der Empfänger in einer selbstwertreduzierenden Abhängigkeit wiederfindet. Natürlich ist die angebotene Hilfe nicht immer adäquat und kann unter gewissen Umständen eher kontraproduktiv als hilfreich sein. Aber nicht nur für den Hilfeempfänger sondern auch für den Helfenden kann soziale Unterstützung in negativen Gefühlen resultieren. So kann über einen längeren Zeitraum gewährte Hilfe für den Unterstützenden eine große Belastung sein. Führen außerdem die Bemühungen $\mathrm{zu}$ keiner erkennbaren Verbesserung der Situation, interpretieren dies Helfende möglicherweise als Beweis, dass der Hilfeempfänger zu wenig Dankbarkeit oder Motivation zeigt, was eine Verweigerung weiterer Hilfe oder Kritik nach sich ziehen kann.

\section{Empirische Befunde zur Bedeutung sozialer Ressourcen in der familiären Pflege}

Bei Durchsicht der Literatur zum Thema soziale Unterstützung im Rahmen der familiären Pflege finden sich uneinheitliche Befunde. In einigen Studien zeigte sich, dass das objektive Ausmaß der Unterstützung (gemessen anhand der Anzahl der Hilfeleistungen und Personen aus dem sozialen Netz) in keinem bedeutsamen 
Zusammenhang $\mathrm{zu}$ der Belastung und der emotionalen Befindlichkeit der Hilfeempfänger steht (Haley, Levine, Brown \& Bartolucci, 1987; Quayhagen und Quayhagen, 1988; Rivera, Rose, Futterman, Lovett \& Gallagher-Thompson, 1991). Andere Autoren fanden wiederum, dass ein stärkeres Ausmaß an sozialer Unterstützung bei den pflegenden Ehepartnern von Alzheimer-Patienten $\mathrm{zu}$ einem geringeren Belastungsempfinden der Angehörigen führte (Clyburn, Stones, Hadjistavropoulos \& Tuokko, 2000; Vitaliano, Russo, Young, Becker und Maiuro, 1991). In einer Studie von Hannappel, Clasyn und Allen (1993) zeigten Pflegende dagegen höhere Depressivitätswerte, je mehr soziale Unterstützung sie erhielten. Diese auf den ersten Blick widersprüchlichen Befunde sind damit zu erklären, dass es in erster Linie auf die subjektive Bewertung der Unterstützung ankommt. Auch besteht keine einheitliche Operationalisierung des Konzeptes der sozialen Unterstützung in den verschiedenen Untersuchungen, was möglicherweise zu den unterschiedlichen Ergebnissen geführt hat. Diese Hypothese wird durch Befunde von Walz und Brown (1991) gestützt, die fanden, dass emotionale Unterstützung durch Anteilnahme und Rücksichtnahme Nahestehender eine positivere Wirkung besitzt als praktische Unterstützung (vgl. auch Pratt, Schmall, Wright \& Cleland, 1985).

Milne, Pitt und Sabin (1993) interessierten sich in ihrer Studie mit 78 pflegenden Angehörigen dafür, wer für potentielle oder tatsächliche Unterstützungsleistungen zur Verfügung steht. Die Teilnehmer nannten als wichtigste Quelle im Bereich der informellen Unterstützung Freunde und Verwandte (81\%), gefolgt von ehrenamtlichen Hilfsdiensten (13\%). Suitor und Pillemer (1996) befragten 95 pflegende Töchter zu den Quellen der sozialen Unterstützung. Für die pflegenden Töchter stellten Geschwister und Freunde die wichtigste Quelle dar. Gleichzeitig zeigte sich aber auch eine sehr große Unzufriedenheit mit den Unterstützungsleistungen der Geschwister. Etwa $66 \%$ der Befragten wünschten sich, dass die Geschwister mehr helfen, 49 \% waren der Meinung, dass ihre Geschwister den Pflegebedürftigen vernachlässigt hätten, ca. $52 \%$ fühlten sich durch die Geschwister kritisiert und $62 \%$ meinten sogar, dass die Geschwister die übernommene Pflege erschweren würden.

Studien von Pagel, Erdly und Becker (1987) sowie Pillemer und Suitor (1996) implizieren darüberhinaus, dass das familiäre soziale Netz eher Stress verursachen kann, je größer die Anzahl der Verwandten ist. Die Autoren argumentieren, dass ein größeres 
familiäres Netz Erwartungshaltungen nach mehr Entlastung bei den Pflegepersonen hervorruft. Wenn diese Hoffnung auf Unterstützung nicht adäquat erfüllt wird, kann dies zu größerer Unzufriedenheit und Belastung führen. Möglicherweise sind die Hauptpflegepersonen eines chronisch Kranken in größeren familiären Netzen außerdem mit mehr unterschiedlichen Werthaltungen und Meinungen bezüglich der Pflege konfrontiert und müssen gegebenenfalls mehr Kritik hinnehmen.

\section{Zusammenfassung}

In der Stresstheorie von Lazarus (1991) wird eine Situation dann als stressreich erlebt, wenn eine Person glaubt, dass die Anforderungen der Umwelt die eigenen Ressourcen übersteigen. Die im Kontext der familiären Pflege am meisten untersuchte Ressource stellt die soziale Unterstützung dar. Soziale Unterstützung kann den Effekt der Stressoren abpuffern. Allerdings ist die subjektive Bewertung und nicht die Quantität der tatsächlich erhaltenen Unterstützung entscheidend für das Entlastungserleben der Pflegenden. Nur so ist zu erklären, dass es widersprüchliche Befunde gibt: Eine Vielzahl von Studien weist einen positiven Zusammenhang zwischen sozialer Unterstützung und Wohlbefinden, Effektivität des Copings und geringeren Depressivitäts- und Angstwerten auf. Jedoch kann die Nutzung sozialer Ressourcen auch einen negativen Effekt haben. Durch die Abhängigkeit von externer Unterstützung können negative Aspekte wie Hilflosigkeit, Gefühle der Unzulänglichkeit und der Verpflichtung in den Vordergrund gerückt werden. Dass soziale Unterstützung auch negative Effekte haben kann, deutet auf eine Schwachstelle im Stressbewältigungsmodell der familiären Pflege von Kramer (1993) hin: Um der Tatsache Rechnung zu tragen, dass Ressourcen im ungünstigen Fall auch zu Stressoren werden können, wie sich besonders eindrücklich im Fall der sozialen Unterstützung zeigt, müssten im Stressbewältigungsmodell Rückkopplungen zwischen den einzelnen Elementen stärker berücksichtigt werden. Trotz aller Einschränkungen, stellt die Unterstützung durch das soziale Netz dennoch eine wichtige Ressource für pflegende Angehörige dar. 


\subsubsection{Copingstrategien}

Eng verbunden mit den zur Verfügung stehenden Ressourcen sind die Copingstrategien; in der Literatur wurden bisher eine Vielzahl diverser Bewältigungsstrategien zusammengetragen, die einen moderierenden Einfluss auf das Belastungsgeschehen haben. Grundsätzlich lassen sich zwei Herangehensweisen an das Konzept unterscheiden. Der einen liegt eine dynamischere, relationale Vorstellung von Copingprozessen zugrunde. Nach diesem Verständnis besitzen Bewältigungsprozesse einen systemischen Charakter und interagieren mit den anderen Komponenten des Stress-Bewältigungsmodells (Bridges, 2003; Nolan, Ingram, \& Watson, 2002). Die andere Forschungsrichtung geht von einem trait-orientierten Modell aus, welches Copingstile als relativ stabile Persönlichkeitsmerkmale versteht. Dies impliziert, dass über verschiedene Kontexte und Situationen hinweg Bewältigungsstrategien oder -formen grundsätzlich generalisiert werden (Haenselt, Danielzik und Waack, 2004).

\section{Empirische Befunde zur Funktion von Copingstrategien bei pflegenden Angehörigen}

Auf der Grundlage empirischer Ergebnisse differenzieren Pearlin, Turner und Semple (1991) drei Funktionen von pflegebezogenem Bewältigungsverhalten, die jeweils an unterschiedlichen Stellen des Stressverarbeitungsprozesses angesiedelt sind: Den Umgang mit der stressreichen Situation selbst, den Umgang mit der Bewertung der Situation sowie den Umgang mit den aus der Situation resultierenden Stresssymptomen. Bei den Bewältigungsstrategien, die direkt an der stressreichen Situation ansetzen, steht im Vordergrund, das Auftreten des Stressors ganz zu verhindern oder ihn in seiner Wirkung zumindest zu verringern. Verhaltensweisen, die dieser Kategorie zuzuordnen wären, sind z. B. die Einbindung einer ambulanten Pflegestation in die häusliche Pflege oder das Ablenken eines Patienten, der perseverierende Äußerungen von sich gibt.

Copingstrategien, die sich auf die emotional-kognitive Bewertung der Stressoren beziehen, haben eine Umdeutung der Gesamtsituation zum Ziel. Beispiele hierfür wären soziale Vergleiche mit anderen Personen in ähnlicher Lage, religiöse Deutungen sowie die Anpassung der Erwartungen an den Patienten an seine tatsächlichen Fähigkeiten. Strategien mit dieser Ausrichtung haben einen moderierenden Charakter und sind auf einen Ausgleich zwischen den objektiven Stressoren und der subjektiv erlebten Belastung gerichtet. 
Der Umgang mit den aus der Situation resultierenden Stresssymptomen richtet sich schließlich darauf, bereits wahrgenommenes Stressempfinden zu vermindern. $\mathrm{Zu}$ konkreten Bewältigungsstrategien in diesem Zusammenhang zählen z. B. die Ausübung von Hobbies, körperliche Betätigung, Ruhepausen oder die Anwendung systematischer Entspannungstechniken wie Autogenes Training. Der effektive Einsatz derartiger Strategien wirkt vorbeugend, sodass aus subjektiv wahrgenommenem pflegebedingten Stress nicht zwangsläufig Einschränkungen des allgemeinen Wohlbefindens im Sinne von Depressivität, Angst oder körperlichen Symptomen resultieren.

Kramer (1993) weist auf eine weitere Kategorie von Copingstrategien hin, nämlich interpersonales Coping. Sie postuliert, dass Coping in Pflegebeziehungen eine andere Qualität hat als Coping in anderen Kontexten, die in der angewandten CopingForschung bislang Gegenstand des Forschungsinteresses waren (z. B. Migräne, Krebserkrankungen, Depressionen). Die Rolle des Pflegenden hat einen starken interpersonalen Charakter, sodass Pflegebeziehungen $\mathrm{zu}$ gestörten affektiven Beziehungen führen können. Interpersonale Konflikte als tägliche Stressoren haben besonders negative Konsequenzen für die emotionale Befindlichkeit (Bolger, DeLongis, Kessler \& Schilling, 1989). Allerdings enthalten die meisten Coping-Messskalen keine interpersonale Dimension.

In Bezug auf die Effektivität der verschiedenen Copingformen, wurden lange Zeit aktive, problemorientierte Copingstrategien als funktional und Wohlbefinden fördernd eingestuft. Emotionsorientierte und vermeidende sowie passiv-kognitive Copingformen wurde dagegen als wenig effektiv betrachtet und es wurde angenommen, dass sie eher depressive und andere negative Reaktionen hervorrufen können (Haley, Levine, Brown \& Bartolucci, 1987; Haley, Levine, Brown, Berry \& Hughes, 1987; Wright, Lund, Caserta \& Pratt, 1991). Erst in den letzen Jahren etablierte sich eine differenziertere Sicht. Inzwischen besteht unter den meisten Autoren Einigkeit darin, dass es nicht sinnvoll ist, einzelne konkrete Bewältigungsstrategien generell als funktional oder dysfunktional einzuordnen. Funktionalität ist in hohem Maße situationsspezifisch und kann nur in Abhängigkeit des Kontextes beurteilt werden (Bridges, 2003; Pearlin, Turner \& Semple, 1989; Wentura, Greve \& Klauer, 2002). So 
kann ein zu aktives Coping in einer Situation, in der es eher angezeigt ist, das Schicksal zu akzeptieren, durchaus negative Folgen haben.

Allerdings konnte wiederholt gezeigt werden, dass für die Situation der Familienpflege generell die Copingstrategien Akzeptieren der Situation, kognitive Umstrukturierung, aktives Problemlösen und Nutzung sozialer Unterstützung günstiger auf das Belastungserleben der Pflegenden auswirkt als Wunschdenken und irrationale Hoffnungen oder Passivität (Brashares \& Catanzaro, 1994; Gunzelmann, 1991; Killeen, 1990; Pruchno \& Resch, 1989b).

\subsubsection{Exkurs: Zusammenhang zwischen Bewältigungsprozessen und Belastungserleben bei pflegenden Angehörigen}

Das Bedingungsgefüge zwischen objektiven Stressoren, dem subjektiv wahrgenommen Stress, den Bewältigungsprozessen und den Konsequenzen der Pflege findet seit Ende der neunziger Jahre in der Caregiving-Forschung stärkere Beachtung. Beispielhaft seien hier die Arbeiten von Lämmler (1998, 2000a, 2000b) erwähnt. Da im qualitativen Teil der vorliegenden Arbeit die Auswertung der Interviews auf der Systematisierung von Bewältigungsprozessen nach Lämmler (1998) basiert, sei an dieser Stelle ein Exkurs eingefügt, in dem die wesentlichen Ergebnisse der Arbeit dargestellt werden.

Als Schema für die Analyse der Bewältigungsstrategien entwickelte Lämmler (1998) auf der Grundlage von qualitativen Interviews ein Inventar. Grundsätzlich lassen sich danach zwei Dimensionen unterscheiden: Bewältigungsstrategien, die 1) in erster Linie der Verbesserung der eigenen Befindlichkeit (ichzentriert) oder 2) der Verbesserung des Zustandes des Patienten (patientenzentriert) dienen. $\mathrm{Zu}$ den Bewältigungsstrategien, die primär eine Verbesserung des Zustandes des Patienten bewirken sollen gehören:

- Pflegerisches Engagement;

- Förderung der Selbständigkeit;

- Kontrolle;

- Zuspruch;

- Stärkung des Kompetenzerlebens;

- Fernhalten psychischer Belastungen; 
- Anpassung der Lebensumwelt;

- Informationssuche;

- Sicherstellen optimaler Therapiebedingungen.

Bewältigungsstrategien, die primär der Verbesserung der eigenen Befindlichkeit dienen, umfassen:

- Akzeptieren;

- positive Umdeutung;

- religiöse Bewältigung;

- Vergegenwärtigen der eigenen Erfolge;

- Erwartungen an den Krankheitsverlauf;

- sozialer Vergleich;

- Distanzierung;

- Erholung;

- Pflege der eigenen Bedürfnisse;

- Suche nach sozialer Unterstützung.

In seiner Untersuchung fand Lämmler zwei charakteristische Typen von pflegenden Angehörigen: Erfolgreiche Bewältigerinnen, die sich dadurch auszeichnen, dass sie Patienten- und ichbezogene Strategien gleichermaßen anwenden, und nicht erfolgreiche Bewältigerinnen, die primär patientenfixierte Strategien wählen. Erfolgreiche Bewältigerinnen sind in der Lage, eigene Bedürfnisse zeitweilig zurückzustellen, wenn es die Umstände erfordern. Sie geben ihnen aber zu gegebener Zeit auch Raum. Ein weiteres zentrales Merkmal der erfolgreichen Bewältigerinnen ist ihre Anpassungsfähigkeit des eigenen Verhaltens an die Fähigkeiten des Patienten. Sie geben soviel Hilfe wie nötig und so wenig wie möglich. Macht der Schlaganfallbetroffene im Rehabilitationsprozess Fortschritte, sind sie in der Lage, ihr Verhalten darauf einzustellen und die Hilfeleistungen $\mathrm{zu}$ vermindern. Kennzeichnend für erfolgreiche Bewältigerinnen ist zudem, dass sie sich von Anfang an nicht ausschließlich mit der Pflegerolle identifizieren. Zwar fühlen sich auch diese Angehörigen belastet, sie besitzen jedoch größere Distanz zu ihren Gefühlen. Dies ermöglicht es ihnen, sich bewusst mit ihren Gefühlen auseinanderzusetzen. Schwierige 
Themen wie Verlust und Sterben oder Aggressionen werden nicht verdrängt. Nicht erfolgreiche Bewältigerinnen vermindern im Gegensatz dazu nicht ihr pflegerisches Engagement, auch wenn Fortschritte in der Rehabilitation $\mathrm{zu}$ verzeichnen sind. Kompetenzen des Patienten werden nicht entsprechend gewürdigt, die Selbständigkeit des Patienten unzureichend gefördert. Diese Angehörigen definieren sich vollkommen über die Pflege, und die Pflegetätigkeit wird zum zentralen Lebensinhalt. Eigene Bedürfnisse werden negiert. Dennoch ziehen dysfunktionale Bewältigerinnen einen psychischen Gewinn aus ihrem Verhalten. Durch die Überidentifikation mit der Pflege gelingt es ihnen, Ängste, Unsicherheiten und Schuldgefühle zu reduzieren. Grundsätzlich identifiziert Lämmler in seiner Studie keine Bewältigungsstrategien, die per se als negativ zu werten waren. Als zentrales Merkmal des funktionalen Copings definiert Lämmler die Flexibilität, auf einen veränderten situativen Kontext auch mit anderen Strategien reagieren zu können (vgl. Kahana, Kahana und Kinney, 1990; Lazarus, 1993a). So geht eine erfolgreiche Bewältigung der Pflegesituation vor allem einher mit der Reduktion pflegerischer Tätigkeiten zugunsten der Förderung selbständigen Verhaltens des Patienten (Lämmler 2000a).

\subsection{Implikationen der bisherigen empirischen Befunde für die vorliegende} Arbeit

Im folgenden Abschnitt sollen die aus den obigen Kapiteln resultierenden Implikationen für die vorliegende Arbeit erörtert werden.

Die in den vorangegangenen Kapiteln dargestellten empirischen Befunde der Caregiving-Forschung zeichnen sich zu einem großen Teil durch ihre Widersprüchlichkeit aus. Lediglich für die langfristigen Konsequenzen der Pflege auf das psychische und physische Wohlbefinden der pflegenden Angehörigen sind die Ergebnisse weitgehend übereinstimmend. Der Eindruck der Uneinheitlichkeit eines Großteils der Befunde ist auf verschiedene Aspekte zurückzuführen, auf die im Folgenden näher eingegangen werden soll.

Komplexität der realen Pflegesituation. Das Forschungsinteresse an der Bedeutung der strukturellen Merkmale des Pflegekontextes führte zu einer Vielzahl von Studien, in denen zum überwiegenden Teil einzelne Kontextmerkmale isoliert betrachtet 
wurden. Wenngleich durch diese Herangehensweise wichtige Erkenntnisse gewonnen werden konnten, wird sie der Komplexität der realen Pflegesituation nicht gerecht. Nicht ausreichend beachtet wurde vielfach, dass bestimmte strukturelle Merkmale mit anderen interagieren. Beispielhaft angeführt sei an dieser Stelle die Studie von PetersDavis, Moss und Pruchno (1999), die nachwies, dass sich Wechselwirkungen zwischen dem Verwandtschaftsgrad und der Qualität der Beziehung in Bezug auf die aus der Pflege resultierende Belastung ergeben (siehe 2.6.1). Werden bei einem Vergleich einzelner Studien mögliche Interaktionen nicht berücksichtigt, können die Ergebnisse inkompatibel erscheinen. Entsprechend ist eine Gegenüberstellung empirischer Befunde nur sinnvoll, wenn die relevanten kontextuellen Merkmale der Pflegesituation in den untersuchten Stichproben weitgehend übereinstimmen.

Methodologische Aspekte. Innerhalb der Caregiving-Forschung besteht weder in Bezug auf die Operationalisierung der zentralen Konzepte noch im Hinblick auf die entsprechenden Messinstrumente Einheitlichkeit. Je nach Erhebung der interessierenden Variablen zeigen sich potentiell unterschiedliche Ergebnisse. So kann zum Beispiel, wie bereits unter 2.6.2 ausführlich dargestellt, soziale Unterstützung sowohl eine positive als auch negative Wirkung bei pflegenden Angehörigen haben, in Abhängigkeit davon, welche Form der sozialen Unterstützung gemessen wurde.

Fehlende Integration empirischer Befunde in theoretische Modelle. Vielfach liegen den vorgestellten Untersuchungen keine expliziten theoretischen Konzepte zugrunde. Eine sinnvolle Analyse der Zusammenhänge der Elemente im Stressbewältigungsprozess kann jedoch nur vor dem Hintergrund theoretischer Modelle erfolgen.

Aus diesen Überlegungen ergeben sich die folgenden Implikationen für die Caregiving-Forschung im Allgemeinen und die vorliegende Studie im Besonderen:

Um der Komplexität der realen Pflegesituation Rechnung $\mathrm{zu}$ tragen, sollten die Kontextvariablen stärker berücksichtigt werden. Eine systematische Analyse der Effekte verschiedener Kontextvariablen ließe sich nur auf der Grundlage einer repräsentativen Stichprobe durchführen. Dies dürfte aus Kostengründen nicht in allen Fällen möglich sein, zumindest sollte dann aber eine reflektierte Auswahl der relevanten Kontextfaktoren erfolgen. Eine Kombination quantitativer und qualitativer 
Forschungsmethoden wird der Komplexität der Thematik am besten gerecht.

Wird eine Vergleichbarkeit mit anderen Studien angestrebt, so sollten im Rahmen eines quantitativen Forschungsansatzes unbedingt standardisierte Messinstrumente verwendet werden. Schließlich sollte eine definitorische Präzisierung der einzelnen Aspekte der familiären Pflege durch die Einbettung der empirischen Ergebnisse in ein theoretisches Modell erfolgen.

\subsection{Interventionsmöglichkeiten zur Verbesserung der Lebenssituation pflegender Angehöriger}

In den bisherigen Kapiteln wurde der Forschungsstand zu den Kontextbedingungen sowie $\mathrm{zu}$ den negativen und positiven Konsequenzen der Pflege dargestellt. In den nächsten Kapiteln geht es um Ansätze zur Prävention der negativen Folgen der Pflege.

Als Konsequenz der empirischen Befunde $\mathrm{zu}$ den Belastungen pflegender Angehöriger wurde eine Vielzahl von psychoedukativen und entlastenden Interventionsprogrammen entwickelt, die darauf abzielten, die negativen Folgen der Pflege für die Angehörigen zu verringern. In Kapitel 2.8.1 wird zunächst ein Überblick über bestehende Interventionsprogramme geboten. Kapitel 2.8.2 stellt die diversen Interventionsangebote in den Kontext der Stresstheorie. Danach wird in Kapitel 2.8.3 die tatsächliche Nutzung von Entlastungsmöglichkeiten in Bezug zum Bedarf beleuchtet. In Kapitel 2.8.4 erfolgt eine Darstellung der empirischen Befunde zur Effektivität von Hilfsangeboten. Abschließend werden methodische Implikationen für die vorliegende Studie reflektiert.

\subsection{1. Übersicht existierender Interventionsmöglichkeiten zur Verbesserung der Lebenssituation pflegender Angehöriger}

Vielfältige Interventionsmaßnahmen wurden in den vergangenen Jahrzehnten entwickelt, die verschiedene Ansätze umfassen und sich anhand diverser Kriterien systematisieren lassen. Biegel et al. (1991) unterteilen die Interventionsprogramme nach Unterstützungsinterventionen (Gesprächs- und Selbsthilfegruppen), psychoedukative 
Interventionen (wissensvermittelnde Kurse), klinisch-therapeutische Interventionen (Psychotherapie, Beratung) sowie Erholungsinterventionen (Tagespflege, ambulante Dienste).

Die vorliegende Arbeit orientiert sich an einer Einteilung von Halsig (1998), die über die oben genannten Interventionsmöglichkeiten hinaus noch andere einbezieht. Eine Übersicht über die verschiedenen Ansatzpunkte der Intervention gibt Abbildung 2. Nachfolgend werden die Interventionstypen kurz erläutert. Die hier vorgestellten Interventionsangebote sind bereichsspezifisch geordnet. In der Praxis bestehen jedoch oft Überschneidungen der einzelnen Bereiche, die zum Teil aufeinander aufbauen. 
1. kurzzeitige/einmalige Informationsvermittlung und Beratung

- Beratungsstellen für pflegende Angehörige

- Beratung durch Hausärzte

- Öffentlichkeitsarbeit über Massenmedien und in Kommunen

2. kontextbezogene und technische Hilfen

- Versorgung mit technischen Hilfsmitteln

- Umgestaltung der Wohnung bzw. Schaffung altersgerechter Wohnungen

3. alltagsbezogene Entlastungen

- ambulante Pflegedienste

- Kurzzeitpflegeeinrichtungen

- Tagesp flegeeinrichtungen

4. psychologische und soziale Hilfsangebote

- psychoedukative Programme

- Gesprächsgruppen für pflegende Angehörige

- Selb sthilfegruppen pflegender Angehöriger

- Einzelberatung durch psychologische Fachkräfte

Abbildung 2: Interventionsmöglichkeiten zur Verbesserung der Lebenssituation von pflegenden Angehörigen

Kurzzeitige/einmalige Informationsvermittlung und Beratung

Seit 1991 sind in Deutschland über 40 Einrichtungen mit dem Aufgabenschwerpunkt der Angehörigenberatung zur Bundesarbeitsgemeinschaft der Beratungsstellen für alte Menschen und ihre Angehörigen (BAGA) zusammengeschlossen (Franke, 2000; Henße \& Ruhnau-Wüllenweber, 1999). Schwerpunkt der Beratungsstellenarbeit ist die 
umfassende Information pflegender Angehöriger über Möglichkeiten der Diagnostik sowie über Therapie- und Entlastungsangebote (z. B. ambulante Pflege- und Entlastungsdienste, Selbsthilfeangebote, technische und pflegerische Hilfsmittel). Darüber hinaus vermitteln Beratungsstellen Wissen über die Rechtsgrundlagen möglicher Hilfen (Bundessozialhilfe-, Krankenkassen- und Pflegeversicherungsrecht). Auch im Vorfeld der Pflegeübernahme bieten gerontopsychologische Beratungsstellen Entscheidungshilfen für Angehörige an.

Die verstärkte Beratung betroffener Familien durch Hausärzte wird immer wieder von fachpolitischen Gruppen in der Altenhilfe gefordert, jedoch findet sie in der Realität eher selten statt (Bruder, 1998). Dies mag zum einen mit dem sehr zeitintensiven Beratungsbedarf pflegender Angehöriger zusammenhängen. Zum anderen scheitert die Beratung Angehöriger hinsichtlich pflegespezifischer Aspekte und Entlastungsmöglichkeiten aber auch am Mangel an geriatrischen und gerontopsychiatrischen Inhalten in der medizinischen Ausbildung. In den vergangenen Jahren gab es jedoch zunehmend Ansätze, Hausärzte stärker in die Aufklärung und Beratung pflegender Angehöriger einzubinden (Larry, 1998).

Auch die Öffentlichkeitsarbeit über Massenmedien und in Kommunen hat zum Ziel, in der Bevölkerung das Bewusstsein für geriatrische Krankheitsbilder wie Schlaganfall oder Demenz einerseits und für Unterstützungsmöglichkeiten der betroffenen Familien andererseits zu verbessern. Neben Informationskampagnen über Printmedien und Fernsehen (z. B. durch die Stiftung Deutsche Schlaganfall-Hilfe oder die Deutsche Alzheimer Gesellschaft) existieren auch Projekte wie das SchlaganfallInformationstelefon (Sterker, 1998) oder das Alzheimer-Telefon der Deutschen Alzheimer Gesellschaft (Jansen, 2007).

\section{Kontextbezogene und technische Hilfen}

Bei den kontextbezogenen und technischen Hilfen geht es um die praktische Erleichterung des Pflegealltags durch die Versorgung mit technischen Hilfsmitteln (z. B. Badewannenlifter, Treppenlifter) oder die Umgestaltung der Wohnung. Zunehmende Verbreitung finden außerdem elektronische Gedächtnishilfen bei Pflegedürftigen mit Gedächtnisproblemen (Kapur, 1995; Wilson, Emslie, Quirk, Evans, 
2001) sowie computergestützte Kommunikationssysteme für Patienten mit einer Aphasie. Während diese Hilfsmittel in erster Linie zur Erleichterung des Alltags für den Patienten konzipiert wurden, tragen sie doch auch erheblich dazu bei, dass die Pflege und Unterstützung des Kranken für die Angehörigen einfacher wird.

\section{Alltagsbezogene Entlastungen}

Hier geht es darum, den Pflegenden durch die Übernahme von Pflegeleistungen zeitlich und aufgabenspezifisch zu entlasten. Dies kann durch tägliche Hausbesuche z. B. von einer Gemeindeschwester, einer ambulanten Pflegekraft oder einem Zivildienstleistenden erfolgen. Eine weitere Möglichkeit ist die vorübergehende Unterbringung des Pflegebedürftigen in einer Kurzzeitpflegeeinrichtung bei Erkrankung oder Kuraufenthalt der Pflegeperson. Kurzzeitpflegestellen sind häufig an Alten- oder Pflegeheime angegliedert.

Tagespflegeeinrichtungen bieten die Möglichkeit, Pflegebedürftige mehrmals pro Woche tagsüber von professionellen Kräften betreuen $\mathrm{zu}$ lassen. Im Leistungsspektrum enthalten sind Hol- und Bringedienste, Mahlzeiten, pflegerische Versorgung, Beschäftigungsangebote sowie therapeutische und rehabilitative Leistungen (Kremer-Preiß \& Zervas, 1994).

\section{Psychologische und soziale Hilfsangebote}

Psychologische und soziale Hilfsangebote umfassen eine große Bandbreite, die von psychoedukativen Interventionen, über Gesprächs- und Selbsthilfegruppen bis zu psychotherapeutischer Einzel- oder Gruppenberatung reicht. Kennzeichen dieser Angebote ist es, dass sie auf einen längeren Zeitraum angelegt sind, mit mindestens 6 Sitzungen. In psychoedukativen Gruppeninterventionen stehen üblicherweise folgende Hauptthemen im Mittelpunkt (vgl. Toseland \& Rossiter, 1989):

- Information über das Krankheitsbild (Erscheinungsbild, Ursachen und Verlauf);

- Vermittlung von Pflegetechniken;

- Information über rechtliche und finanzielle Unterstützungsmöglichkeiten;

- Information über bestehende Entlastungseinrichtungen (ambulante Dienste, Heime);

- Informationen über die Auswirkungen der Pflege auf die Pflegeperson; 
- Vermittlung von Maßnahmen zur Selbstpflege.

Gesprächs- und Selbsthilfegruppen, die wie die psychoedukativen Interventionsprogramme oft an Kliniken oder gerontopsychologischen Beratungsstellen angegliedert sind oder auch von Betroffenen-Organisationen (z. B. Stiftung Deutsche Schlaganfall-Hilfe) angeboten werden, sind schwerpunktmäßig auf emotionale Unterstützung sowie Erfahrungsaustausch in Bezug auf den Umgang mit der Pflegesituation ausgerichtet. Diese Gruppen existieren oft sogar über einen Zeitraum von einigen Jahren.

Psychotherapeutische Einzel- oder Gruppenberatung durch entsprechend geschulte Fachkräfte wird vereinzelt an Beratungsstellen für ältere Menschen und ihre Angehörigen oder auch an Fachkliniken angeboten. In einigen Fällen schließt die Intervention auch ein Therapie- und Förderprogramm für die Pflegebedürftigen ein (Auer, Donabauer, Zehetner \& Span, 2007; Jost, Voigt-Radloff, Hüll, Dykierek, \& Schmidtke, 2006). Ziel des psychoedukativen Schulungsangebotes für die Angehörigen ist zum einen die Stärkung ihrer Ressourcen und zum anderen die Modifikation ihres Erlebens und Verhaltens (Wilz, Adler \& Gunzelmann, 2001). Neben klientenzentrierten Verfahren kommen dabei auch kognitive sowie verhaltenstherapeutische Verfahren zum Einsatz. Psychotherapeutische Ansätze in der Angehörigenarbeit zeichnen sich dadurch aus, dass sie auf die Pflegesituation fokussiert, lösungsorientiert und multimethodal im Vorgehen sind (Wendel, 2005). In vielen Fällen sind die Grenzen zwischen Beratung und Psychotherapie - wie auch in anderen psychosozialen Bereichen - fließend.

\subsubsection{Interventionsangebote für Angehörige im Kontext der Stresstheorie}

Im folgenden Abschnitt werden theoretische Überlegungen zur Wirkweise verschiedener Interventionsangebote vorgestellt. Dabei werden die Interventionsmöglichkeiten in Beziehung gesetzt zum unter 2.5 beschriebenen Stressmodell.

Legt man die stresstheoretischen Überlegungen von Lazarus und Folkman (1984) zugrunde, lassen sich die im obigen Abschnitt dargestellten 
Interventionsmöglichkeiten danach kategorisieren, auf welches Element innerhalb des Stressprozesses sie abzielen und welche Komponente modifiziert werden soll (Kahana \& Kinney, 1991; Zarit \& Leitsch, 2001). Die modifizierbaren Elemente des Stressprozesses umfassen die Stressoren selbst, die subjektive Bewertung der Stressoren sowie die Ressourcen, die für die Stressbewältigung zur Verfügung stehen.

Wie oben bereits ausführlich beschrieben, betrachten Lazarus und Folkmann (1984) Stress als eine Folge von Interaktionen zwischen den objektiven Gegebenheiten in der Umwelt, den subjektiven Bewertungen dieser Ereignisse durch das Individuum, den Bewältigungsversuchen sowie den verhaltensbezogenen und psychosozialen Konsequenzen. Abbildung 3 verdeutlicht, an welchen Punkten im Stressprozess Interventionen ansetzen können. Das Schema ist dabei stark vereinfacht; nicht berücksichtigt sind Interaktionen, die zwischen den einzelnen Elementen stattfinden können. So ist auch die Zuordnung einer Intervention zu den einzelnen Komponenten des Stressprozesses eher als schwerpunktmäßige Einteilung zu verstehen, da einige Interventionsmaßnahmen auch auf andere Elemente des Modells Einfluss haben. 


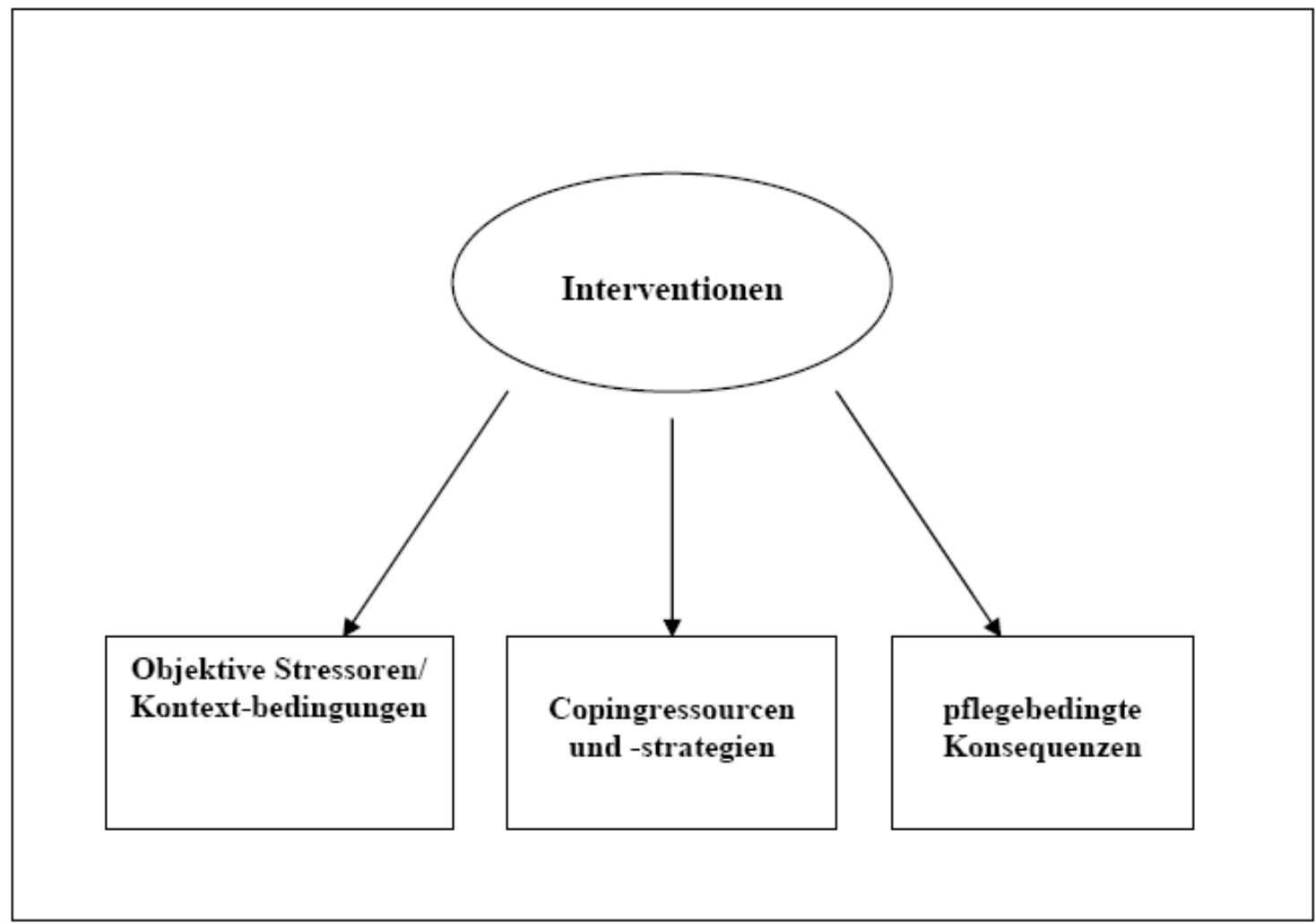

Abbildung 3: Wirkweise der Interventionsansätze im Kontext der Stresstheorie (modifiziert nach Kahana \& Kinney, 1991)

Interventionen, die an den objektiven Stressoren ansetzen, sind beispielsweise alltagsbezogene Hilfen wie Tagespflege oder ambulante Pflege. Die Übernahme von Pflegetätigkeiten durch die jeweiligen Dienste entlastet Angehörige in ihren pflegebezogenen Aufgaben. Aber auch technische Hilfen können dazu beitragen, die Kontextbedingungen $\mathrm{zu}$ optimieren und dadurch die objektiven Stressoren $\mathrm{zu}$ vermindern. So kann eine externe Gedächtnishilfe wie ein Pager oder ein Palm einen Schlaganfallpatienten mit schweren Gedächtniseinbußen bei der Strukturierung seines Tagesablaufs unterstützen und damit die Angehörigen von der ständigen Erinnerung an bestimmte Aktivitäten befreien (Kapur, 1995; Wilson et al. 2001). Präventive Maßnahmen wie die Beratung vor Übernahme einer Pflege setzen ebenfalls an den objektiven Stressoren an, indem zum Beispiel den Angehörigen Möglichkeiten aufgezeigt werden, die pflegebezogenen Aufgaben schon früh im Pflegeprozess zu verringern, etwa durch Einbeziehung einer ambulanten Pflegestation. 
Interventionen, die auf das zweite Element des Modells, den Copingressourcen und -strategien abzielen, sollen in erster Linie eine Veränderung der subjektiven Bewertung der objektiven Stressoren herbeiführen. So kann die Verbesserung und Intensivierung von Informationen entscheidend dazu beitragen, die subjektive Bewertung objektiver Stressoren zu verändern. Längsschnittstudien mit pflegenden Angehörigen von Demenzpatienten bestätigen, dass Veränderungen in der Bewertung demenzbezogenen Verhaltens einen positiven Einfluss auf das Stressempfinden haben (Aneshensel, Pearlin, Mullan, Zarit \& Whitlatch 1995; Zarit, Todd \& Zarit, 1986) Beispielsweise kann der Ehemann einer Schlaganfallbetroffenen mit Aufmerksamkeitsdefiziten möglicherweise besser mit der Pflegesituation umgehen, wenn er in einem psychoedukativen Kurs darüber aufgeklärt wurde, dass diese Beeinträchtigungen krankheitsbedingt sind und sich nicht etwa willentlich gegen ihn richten. Das erworbene Wissen (eine psychologische Ressource) ermöglicht also einen adäquateren Umgang mit potentiell stressauslösenden Situationen.

Selbsthilfe- und Gesprächsgruppen für Angehörige bieten in erster Linie emotionale Unterstützung, die sich modifizierend auswirkt auf die Beziehung zwischen subjektiv wahrgenommenem Stress und den längerfristigen Konsequenzen der pflegebedingten Belastung. Als besonders wirksam für die Umbewertung der eigenen Situation hat sich die Copingstrategie des sozialen Vergleichs erwiesen, die Angehörige oft im Rahmen von Gesprächsgruppen anwenden. Durch die Erkenntnis, dass es noch schlimmere Schicksale als das eigene gibt, relativiert sich für die Angehörigen häufig der subjektiv wahrgenommene Stress.

Die Vermittlung von Maßnahmen zur Selbstpflege wie Entspannungstraining oder sportliche Aktivitäten im Rahmen von Kursen oder Gesprächsgruppen für Angehörige wiederum zielen darauf ab, die langfristigen negativen Konsequenzen der pflegebedingten Belastung $\mathrm{zu}$ verhindern oder bereits eingetretene Belastungssymptome $\mathrm{zu}$ verringern. Auch die psychotherapeutische Behandlung von Angst oder Depressionen fällt in diese Kategorie.

Zusammenfassend lässt sich also festhalten, dass sich die modifizierbaren Aspekte des Stressprozesses durch verschiedene Interventionsansätze verändern lassen. 
Die verschiedenen Möglichkeiten der Intervention für pflegende Angehörige lassen den Eindruck entstehen, dass der Bedarf an Entlastung durch die vorhandenen Angebote gedeckt werden kann. Allerdings besteht in der Praxis ein Missverhältnis zwischen dem Bedarf und der tatsächlichen Nutzung der Unterstützungsmöglichkeiten durch Angehörige. Darauf soll im folgenden Abschnitt ausführlich eingegangen werden.

\subsubsection{Nutzung von Unterstützungsangeboten vs. Entlastungsbedarf}

Trotz einer zunehmenden Bandbreite unterschiedlichster Unterstützungsangebote nutzen viele pflegende Angehörige, die von diesen Angeboten profitieren könnten, diese nicht oder nur für kurze Zeit (Kosloski \& Montgomery, 1994; Lieberman \& Kramer, 1991; Montgomery \& Kosloski, 1995). Zarit und Leitsch (2001) verweisen darauf, dass das Problem der mangelnden Inanspruchnahme noch eine der großen unbeantworteten Fragen in der Angehörigenforschung ist.

Betroffen von geringer Inanspruchnahme in Relation $\mathrm{zu}$ den potentiellen Nutzern sind nahezu alle Interventionsprogramme. So besucht in Berlin nur ein Bruchteil der pflegenden Angehörigen psychoedukative Kurse, was auch für andere Städte gilt (Holz, 1998). In Berlin konnten im Jahr 1998 von 81 geplanten Kursen 26 aufgrund $\mathrm{zu}$ geringer Anmeldungen nicht stattfinden (Bestandsaufnahme durch das Berliner Service- und InformationsZentrum für Angehörigenarbeit im Sachbericht des BeSIZ, 1998). Auch in Bezug auf die tatsächliche Nutzung von alltagsbezogenen Entlastungen ergeben sich Diskrepanzen zum ermittelten Unterstützungsbedarf: Eine repräsentative Studie (Halsig, 1998), in der 406 Hauptpflegepersonen mittels eines umfangreichen Leitfadens befragt wurden, ergab eine nur geringe Nutzung von ambulanten sozialen Diensten oder Organisationen. Am häufigsten war die Inanspruchnahme von personellen Hilfsangeboten: Gemeindeschwestern, Krankenpfleger, oder Zivildienstleistende wurden insgesamt von 20,3 \% der Befragten in Anspruch genommen. In nur 3,8 \% der Fälle wurden sachliche Hilfen genutzt (Essen auf Rädern, ambulante Fahrdienste). Im Pflegebericht des Medizinischen Dienstes der Spitzenverbände der Krankenkassen zeigt sich, dass sogar Angehörige von Pflegebedürftigen in der Stufe III „,schwerstpflegebedürftig“ nur zu 40\% Sachleistungen in Anspruch nehmen (MDS, 1996). 
Befragt man Angehörige zu ihrem Bedarf an Entlastung, zeigt sich, dass sie sich durchaus mehr Unterstützung wünschen, wenn auch nicht nur in Form von professioneller Hilfe. In einer repräsentativen Studie von Gräßel (1997) mit 1911 Pflegepersonen wurden unter anderem individuelle Wünsche der Pflegepersonen nach Entlastung ermittelt. $53 \%$ der Pflegenden wünschten sich (mehr) nicht-professionelle praktische Hilfe, z. B. durch Freunde, Verwandte oder Nachbarn. $44 \%$ forderten mehr professionelle praktische Hilfe bei der Pflege, z. B. durch Sozialstationen, Tagespflege oder Kurzzeitpflege. 69 \% wünschten sich (mehr) Anerkennung der Pflegetätigkeit, sei es in ideeller oder materieller Form. $25 \%$ hatten Bedarf an zusätzlicher medizinischer Information und Beratung durch einen Haus- oder Facharzt.

Die Gründe, die Angehörige für die Nichtinanspruchnahme von Unterstützungsangeboten anführen, sind vielfältig. Dazu zählen: Den Kranken nicht mit einem Fremden allein lassen zu wollen, eingeschränkte finanzielle Möglichkeiten oder die Angst, dass der Kranke sich zu sehr aufregen könnte (Gwyther, 1989). Einer der meistgenannten Gründe ist die Einschätzung, dass das Entlastungsangebot keinen Nutzen bringt (Caserta, Lund, Wright \& Redburn, 1987; Rakowski und Clark, 1985). Halsig (1998) ermittelte in seiner Studie ebenfalls die Gründe für die Nichtnutzung von Diensten. Für $17 \%$ der Befragten spielten finanzielle Erwägungen eine entscheidende Rolle. Weiterhin wurden familiäre Hemmungen (9 \%), ein zu hoher Organisationsaufwand (9\%) und eine unzureichende Hilfe durch die sozialen Dienste (5\%) genannt.

Nach Brodaty und Gresham (1992) lassen sich die Hinderungsgründe für die Inanspruchnahme sozialer Dienste in drei Aspekte unterteilen: 1. Auf die Pflegeperson bezogene Hindernisse (zu hohe Kosten, sozialer Druck, Schuldgefühle gegenüber dem Angehörigen); 2. auf den Pflegebedürftigen bezogene Hindernisse (die Symptomatik des Patienten, z. B. aggressives Verhalten, Immobilität, lässt eine Nutzung nicht zu) sowie 3. strukturelle Hindernisse (räumliche Entfernung, Inflexibilität der Entlastungsangebote, lange Wartezeiten, mangelnde Kenntnis von Angeboten).

In Bezug auf den letztgenannten Aspekt vermutet Holz (1998) jedoch, dass diese Gründe häufig von Angehörigen vorgeschoben werden, um die Nichtnutzung entsprechender Entlastungsangebote zu rechtfertigen. Sie postuliert, dass stattdessen vor allem kognitive und emotionale Barrieren eine entscheidende Rolle spielen. Dazu 
gehören internalisierte Normen und Einstellungen, die pflegende Angehörige davon abhalten, familiäre Probleme außerhalb des Familienkreises zu „veröffentlichen“. Dabei sind biographische Faktoren von Bedeutung; in vielen Fällen stammen pflegende Angehörige aus der älteren Generation, die Probleme in der Familie nicht öffentlich macht und die es als Schwäche ansieht, Hilfe von außen anzunehmen. Hilfe oder Unterstützung durch Dritte wird von den Pflegenden als Ungenügen oder Versagen der eigenen Kräfte erlebt und kann zu Schuldgefühlen führen (Bruder, 1998). Jansen (1999) führt außerdem an, dass vielfach die Inanspruchnahme von Hilfen auf Grund von „unsichtbaren Loyalitäten“" als Verrat erlebt würde und es Angehörigen daher schwer falle, die Verantwortung in der Pflege zu teilen oder abzugeben.

Darüber hinaus kann das Gefühl der eigenen Unersetzlichkeit Pflegende daran hindern, Hilfe von außen anzunehmen. Dies mag vor allem auf Angehörige zutreffen, die sich die Pflege zum sinnstiftenden und bedeutsamsten Lebensinhalt werden ließen und aus dieser Aufgabe ihre - vielleicht einzige und wichtigste - Bestätigung ziehen. Auch das Leugnen von Beeinträchtigungen beim Gepflegten kann die Inanspruchnahme von Entlastungsangeboten verhindern. In einigen Fällen ist $\mathrm{zu}$ beobachten, dass Angehörige das tatsächliche Ausmaß der Beeinträchtigungen ihres kranken Familienmitgliedes negieren, da dies für sie eine Bedrohung darstellt. Das Leugnen objektivierbarer Beeinträchtigungen hat zur Konsequenz, dass Entlastungsangebote nicht für notwendig erachtet und daher auch nicht angefordert werden (Zarit \& Leitsch, 2001). Die Angst vor Kontrollverlust und vor Abhängigkeit von anderen kann eine weitere Barriere darstellen, Hilfe in Anspruch $\mathrm{zu}$ nehmen. Das Erleben von Eigenkompetenz in Problemsituationen trägt entscheidend zur Aufrechterhaltung der Handlungsfähigkeit und des Selbstwertgefühls bei. Die Inanspruchnahme von Hilfe kann dagegen als Bedrohung der Selbstachtung erlebt werden. Schließlich können soziale Ängste die Annahme von Hilfen erschweren. So werden Gruppenangebote von sozial ängstlichen Angehörigen eher als stressfördernd denn als Entlastung oder Hilfe erlebt. Besonders Angehörige mit niedrigem Bildungsniveau und geringem Einkommen hegen zudem Befürchtungen, nicht verstanden zu werden oder unterlegen zu sein (Holz, 1998).

Die Analyse der Bedingungen, die Angehörige davon abhalten, Hilfe in Anspruch zu nehmen, wird von einigen wenigen Studien ergänzt, die sich mit der Frage 
befassen, welche Faktoren dazu beitragen, dass Angehörige Hilfen annehmen. Übereinstimmend mit Hamilton, Brown, Kerber, Thurlow und Schwieterman (1996) stellte Gräßel (1997) fest, dass die objektive und subjektive Belastung der Pflegenden entscheidende Prädiktoren darstellen für die Nutzung von Unterstützungsangeboten. Gräßel fand darüber hinaus, dass der Mangel an familiärer Unterstützung ein weiterer Prädiktor war. Hamilton und Mitarbeiter (1996) nennen als zusätzlichen Prädiktor für die Annahme von Unterstützung das Gefühl von Kompetenz (mastery); nahm dieses ab, so stieg die Bereitschaft der Angehörigen, professionelle Hilfe in Anspruch zu nehmen.

Zusammenfassend lässt sich feststellen, dass Angehörige allzu häufig ohne professionelle Unterstützung bis an die Belastungsgrenze und sogar darüber hinaus pflegen. Ergebnisse qualitativer Untersuchungen haben gezeigt, dass sich viele Angehörige nicht helfen lassen, bis ihre körperlichen und psychischen Reserven bereits erschöpft sind. Eine zu späte oder unzureichende Inanspruchnahme professioneller Hilfen kann sich jedoch sowohl für den Pflegenden als auch für den Pflegebedürftigen negativ auswirken: Die dauerhafte psychische und körperliche Belastung der Pflegeperson kann ein Gefühl der Überforderung zur Folge haben, was wiederum im Kontext familiärer Konflikte zu Gewalt gegenüber dem Pflegebedürftigen führen kann (Thoma, Schacke \& Zank, 2004). Darüber hinaus kann mangelnde Information des pflegenden Angehörigen zu einer unsachgemässen Pflege des Kranken führen. Werden Unterstützungsangebote erst in Anspruch genommen, wenn schon eine chronifizierte Überforderungssituation besteht, können Angehörige vielfach nicht mehr davon profitieren (Bookwala, Zdaniuk \& Burton, 2004; Schmall, 1995).

\subsubsection{Evaluation von Interventionsmöglichkeiten: Empirische Befunde}

Das folgende Kapitel beschäftigt sich mit der Evaluation von Interventionsprogrammen. Zunächst werden einige grundsätzliche Überlegungen zu Evaluationsstudien angestellt. Dann werden empirische Ergebnisse zur Effektivität verschiedener Interventionsangebote im Bereich der Angehörigenarbeit zusammengefasst. Die Gliederung orientiert sich an der oben beschriebenen Systematisierung, angelehnt an Halsig (1998). Es werden jeweils beispielhaft Evaluationsstudien zu Interventionen aus 
den Bereichen 1. kurzzeitige/einmalige Informationsvermittlung und Beratung, 2. kontextbezogene und technische Hilfen, 3. alltagsbezogene Entlastungen sowie 4. psychologische und soziale Hilfsangebote dargestellt. Aus den methodischen und konzeptionellen Problemen der Evaluationsstudien werden im nachfolgenden Kapitel Implikationen für die vorliegende Arbeit abgeleitet.

Der personelle und finanzielle Aufwand, der in den vergangenen Jahren für die Entwicklung und Durchführung von Interventionsprogrammen bereitgestellt worden ist, übersteigt laut Zarit und Leitsch (2001) die Sorgfalt, mit der die entsprechenden Angebote evaluiert wurden, um ein Vielfaches. Gemessen an der Menge der existierenden Unterstützungsangebote ist die Anzahl der publizierten Evaluationsstudien nach wie vor sehr klein. Am besten untersucht sind bislang verschiedene psychoedukative Programme.

Die wenigen empirischen Befunde zeigen, dass Interventionen, die sorgfältig entwickelt und implementiert wurden, durchaus pflegenden Angehörigen nutzen. So kann z. B. Kurzzeitpflege das Stressempfinden Angehöriger entscheidend reduzieren (Montgomery \& Borgatta, 1989; Zarit, Stephens, Townsend \& Greene, 1998). Auch individuelle Beratung und Familienberatung können die Belastung Angehöriger vermindern und unter Umständen sogar eine Institutionalisierung des Patienten verhindern oder aufschieben (Marriott, Donaldson, Tarrier \& Burns, 2000; Mittelman, Ferris, Shulman, Steinberg \& Levin, 1996; Mittelman, Roth, Clay \& Haley, 2007).

Insgesamt unterliegt die Evaluationsforschung im Bereich der Angehörigenarbeit nach wie vor methodischen Problemen. Nachweise der Effektivität der Interventionen werden selten mittels standardisierter Messinstrumente erzielt, sondern häufiger durch Rückgriff auf den klinischen Eindruck und Verwendung unstandardisierter Fragebögen. In vielen Fällen wurden keine Längsschnittstudien, sondern einmalige Messungen durchgeführt (Toseland \& Rossiter, 1989). Außerdem fehlt es oft an angemessenen Strategien für die Evaluation von Programmen (Zarit \& Leitsch, 2001). So wird viel zu selten berücksichtigt, dass die Methoden, die z. B. bei klinischen Medikamentenuntersuchungen angewandt werden, für die Evaluation von Interventionen, die Veränderungen des Verhaltens zum Ziel haben, nicht adäquat sind. Obwohl in der Forschung oft ein randomisierter Versuchsaufbau als Goldstandard 
angesehen wird, kann dieser Ansatz unter Umständen die interne und externe Validität stärker beeinträchtigen als ein alternativer Versuchsaufbau. Auch werden in vielen Fällen die Ziele der Evaluationsstudien im Bereich der Angehörigenarbeit zu hoch gesteckt. Denn es ist nicht zu erwarten, dass eine Intervention drastische Veränderungen für den Angehörigen mit sich bringt. Verhaltensbezogene Interventionen können den Verlauf einer Erkrankung nicht aufhalten, auch können sie manche Aspekte der Pflegesituation, z. B. das Verlustempfinden von Angehörigen kognitiv beeinträchtigter Personen, nicht vermindern. Manche Interventionen zeigen daher nur subtile Veränderungen oder sie haben einen verzögerten Effekt (Ostwald, Hepburn, Caron, Burns \& Mantell, 1999). In einigen Fällen könnte ein positiver Effekt bedeuten, dass sich die Situation für die Treatmentgruppe im Vergleich zu einer Kontrollgruppe nicht verschlimmert.

Diese grundsätzlichen methodischen Überlegungen werden im nächsten Kapitel ausführlich aufgegriffen, nachdem in den folgenden Abschnitten der Stand der Evaluationsansätze für die einzelnen Interventionsbereiche dargestellt worden ist.

\section{1) Kurzzeitige/einmalige Informationsvermittlung und Beratung}

In den Beratungsstellen für ältere Menschen und ihre Angehörigen gehören mittlerweile statistische Erhebungen zum Standard, jedoch werden meist lediglich soziodemographische Daten sowie Aspekte des Nutzungsverhaltens der Ratsuchenden ermittelt (Franke, 2000; Bundesarbeitsgemeinschaft der Beratungsstellen für alte Menschen und ihre Angehörigen [BAGA], 1999). Begleitstudien umfassen in einigen Fällen auch Einzelfallschilderungen oder qualitative Untersuchungen. Empirische Ergebnisse darüber, ob und welchen Effekt die Vermittlung von Informationen über Diagnostik, Therapie und psychosoziale Unterstützungsmöglichkeiten auf die pflegenden Angehörigen hat, sind jedoch rar.

Eine methodisch gelungene Studie von Lomer und McLellan (1987) befasste sich mit den Effekten der Verteilung von Informationsmaterial an 46 Schlaganfallpatienten und ihre Angehörigen mit ausführlichen Informationen zur Ätiologie, Therapie, psychosozialen Hilfen sowie finanziellen Hilfen. Sie fanden, dass 
Patienten und Angehörige, die das Informationsmaterial erhalten hatten, mehr über die Ätiologie und Risikofaktoren des Schlaganfalls wussten als die Kontrollgruppe. In Bezug auf den Wissensstand über die Prognose oder die Möglichkeiten psychosozialer Unterstützung war jedoch kein signifikanter Unterschied festzustellen. Pain und McLellan (1990) untersuchten die Effektivität von personifiziertem Informationsmaterial und mussten feststellen, dass nur $50 \%$ der Teilnehmer das Material hilfreich fanden. Des Weiteren konnten keine signifikanten Unterschiede in Bezug auf schlaganfallbezogenes Wissen, Lebenszufriedenheit und Aktivitäten des täglichen Lebens ermittelt werden. Allerdings ließ sich bei den Angehörigen in der Treatmentgruppe eine geringere psychische Symptomatik nachweisen als bei der Kontrollgruppe. Kritisch anzumerken ist, dass die Gesamtstichprobe in dieser Studie relativ klein war $(\mathrm{N}=36)$.

\section{2) Kontextbezogene und technische Hilfen}

Evaluationsstudien, die sich mit der Versorgung mit technischen Hilfsmitteln befassen, sind in der Regel auf die Patienten ausgerichtet, da die entsprechenden Interventionsmaßnahmen in erster Linie konzipiert wurden, um den Betroffenen den Alltag zu erleichtern. Inwiefern Angehörige von Hilfsmitteln profitieren, ist eine Fragestellung, die in zukünftigen Studien untersucht werden könnte.

\section{3) Alltagsbezogene Entlastungen}

\section{Ambulante Pflegedienste}

Berücksichtigt man, dass ambulante Pflegedienste einen erheblichen Kostenfaktor für die Pflegeversicherung darstellen, ist es erstaunlich, dass nach Wissen der Autorin bislang keine Studien zu der Frage existieren, ob und inwiefern pflegende Angehörige ambulante Pflegedienste als Entlastung empfinden. Aufgrund von Erfahrungen aus der Praxis muss jedoch konstatiert werden, dass die durch ambulante Pflegedienste gebotene Entlastung oft nicht den Erwartungen der pflegenden Angehörigen entspricht. Als Gründe werden von den Angehörigen die hohe Fluktuation und zeitliche Unzuverlässigkeit sowie die zum Teil ungenügende Ausbildung der Einsatzkräfte genannt. Dies führt in einigen Fällen dazu, dass Angehörige ambulante Pflegedienste eher als zusätzlichen Belastungsfaktor empfinden und die Pflege aus diesem Grund 
nach einer gewissen Zeit wieder vollständig selbst übernehmen.

\section{Kurzzeitpflege}

Studien, die sich mit der Effektivität der Kurzzeitpflege befassen, werden bisher in vielen Fällen dem methodischen Anspruch an Evaluationsstudien nicht gerecht. Die meisten Arbeiten sind rein deskriptiv, nur selten wird ein LängsschnittKontrollgruppen-Design verwendet. Als Erfolgskriterien wurden die Zufriedenheit mit dem Angebot, die objektive und subjektiv wahrgenommene Belastung, die Stabilität der häuslichen Situation sowie das allgemeine Wohlbefinden erfasst. In einer der wenigen methodisch sorgfältig durchgeführten Studien von Burdz, Eaton und Bond (1988) wurden die Auswirkungen von stationärer Kurzzeitpflege auf demente und nicht demente Patienten sowie auf deren pflegende Angehörige untersucht. Die Autoren konnten nachweisen, dass Pflegende von funktional beeinträchtigten Patienten stärker von der professionellen Pflege profitierten als Angehörige von Demenzkranken. In Bezug auf die subjektive Belastung zeigte sich allerdings kein Unterschied zwischen der Treatmentgruppe und einer Kontrollgrupppe, obwohl die Angehörigen in der Treatmentgruppe das Angebot größtenteils als „,sehr hilfreich“ erlebten.

Eine differenzierte Untersuchung der verschiedenen Effektbereiche von Kurzzeitpflege nahmen Scharlach und Frenzel (1986) vor. Die Mehrheit der Angehörigen, die dieses Angebot in Anspruch nahmen, nutzten die freie Zeit, um sich „körperlich und seelisch auszuruhen“ (81 bzw. 77 \%). 34 \% der Angehörigen gaben außerdem an, familiäre (34 \%) und außerfamiliäre Kontakte (26 \%) verstärkt zu pflegen. Darüber hinaus waren ca. $75 \%$ der pflegenden Angehörigen davon überzeugt, dass sich ihre Gesundheit durch die Nutzung der Kurzzeitpflege verbessert habe. 56 \% glaubten außerdem, dass sich das Verhältnis zum Pflegebedürftigen durch die vorübergehende räumliche Trennung entspannt habe. Die Möglichkeit, Kurzzeitpflege in Anspruch zu nehmen, erleichterte $84 \%$ der Angehörigen, die Pflege aufrechtzuerhalten. Allerdings meinten nur ca. $30 \%$, dass eine langfristige Heimunterbringung durch die Nutzung der Kurzzeitpflege weniger wahrscheinlich sei. Für ein Drittel der Angehörigen war die Kurzzeitpflege eine so positive Erfahrung, dass eine langfristige Institutionalisierung von ihnen sogar eher in Betracht gezogen würde. Als Begründung führten viele dieser Angehörigen an, dass der Abstand von der Pflegesituation ihnen erst das ganze Ausmaß 
ihrer Belastung und Einschränkung bewusst gemacht habe.

Tagespflege

Auch für den Bereich der Tagespflege lassen sich kaum Studien finden, denen ein Längsschnitt-Kontrollgruppen-Design zugrunde liegt. Vielfach werden unstandardisierte Maße zur Überprüfung der Interventionseffekte verwendet, die sich auf die Zufriedenheit mit dem Angebot, die objektive und subjektive Belastung durch die Pflege sowie auf das allgemeine Wohlbefinden beziehen.

In einer Untersuchung zu den Entlastungseffekte durch Tagespflege fanden Berry, Zarit und Rabatin (1991) ein unerwartetes Ergebnis: In ihrer Befragung pflegender Angehörige zum zeitlichen Umfang der Pflege stellte sich überraschenderweise heraus, dass Angehörige an Tagen, an denen sie Tagespflege nutzten, mehr Zeit für die Pflege benötigten, als an anderen Tagen. Begründet wurde dies mit dem erhöhten Aufwand, der erforderlich sei, um den Pflegebedürftigen für den Aufenthalt in der Tagespflege (Körperpflege, Ankleiden, Transport) vorzubereiten.

Meister und Zehle (2003) konnten dagegen in einer quasiexperimentellen Studie zeigen, dass sich bereichsspezifische Entlastungseffekte durch die Tagespflege nachweisen ließen. Die Angehörigen mit Pflegebedürftigen in einer Tagespflegestätte profitierten im Vergleich zur Kontrollgruppe in folgenden Bereichen: Motivieren und Anleiten, Bedürfniskonflikte sowie gesundheitliche Beeinträchtigung.

Eine der methodisch sorgfältigsten Studien zur Evaluation von Tagespflege wurde von Zarit, Stephens, Townsend und Greene (1998) durchgeführt. Anhand eines quasi-experimentellen Längsschnittdesigns überprüften die Autoren Interventionseffekte für spezifische Belastungsindikatoren sowie für das allgemeine Wohlbefinden der Pflegenden. Beim Posttest nach 3 Monaten ließen sich positive Effekte hinsichtlich subjektiver Überforderungsgefühle (overload), Angst/Besorgnis, Depressivität und Ärger nachweisen. Keine Auswirkung hatte die Nutzung der Tagespflege auf die Dimension Rollenzwang (role captivity) sowie auf positive Gefühle in Bezug auf die Pflege. Beim zweiten Posttest sechs Monate später zeigten sich nach wie vor positive Effekte in Bezug auf subjektive Überforderungsgefühle und depressive Symptome.

In einer ebenfalls methodisch sehr aufwändigen Studie von Schacke (2000) zur Entlastungsfunktion gerontopsychiatrischer Tagesstätten wurden 37 Angehörige von 
demenzkranken Tagesstättenbesuchern befragt. Im Rahmen eines LängsschnittKontrollgruppen-Designs kamen sowohl quantitative als auch qualitative Verfahren zum Einsatz. Die Ergebnisse zeigten, dass sich Entlastungseffekte vor allem für wahrgenommene Konflikte zwischen familiären, beruflichen und pflegerischen Anforderungen ergaben. Auch war eine Verbesserung der sozialen Aktivitäten und der wahrgenommenen Belastung durch stereotype Verhaltensweisen der Patienten zu verzeichnen. Keine Interventionseffekte ließen sich dagegen für das allgemeine Wohlbefinden der Pflegenden nachweisen. Schacke (2000) führt als Erklärung an, dass Lebenszufriedenheit, Depression und Selbstwert der Angehörigen von vielen außerhalb des Pflegekontextes angesiedelten Aspekten beeinflusst wird, sodass die Belastungsreduktion durch die Intervention nur geringfügig ist.

Lawton, Brody und Saperstein (1989) untersuchten im Rahmen einer längsschnittlichen Kontrollgruppenstudie die Effekte einer Kombination verschiedener alltagsbezogener Entlastungen (Betreuung des Pflegebedürftigen zu Hause, Besuch einer Tagespflegeeinrichtung, Kurzzeitpflege). Insgesamt zeigten sich keine positiven Interventionseffekte in Bezug auf subjektive Belastung, Depressivität, psychiatrische Symptomatik oder subjektive Gesundheitseinschätzung der Pflegenden. Kritisch anzumerken ist jedoch, dass viele Angehörige in der Treatmentgruppe die verfügbaren (kostenlosen) Angebote gar nicht (50\%) oder nur sehr selten in Anspruch nahmen. So wurde eine Tagesstätte im Median nur 10 Tage pro Jahr von den betroffenen Familien genutzt. Gleichzeitig nutzten Angehörige in der Kontrollgruppe vielfach andere Interventionsangebote.

Ein ähnliches Problem wie in der Studie von Lawton, Brody und Saperstein (1989) zeigte sich in einer Untersuchung von Montgomery und Borgatta (1989). Sie überprüften die Wirkung unterschiedlicher Kombinationen von Unterstützungsangeboten (psychoedukative Seminare, Unterstützungsgruppen, Familienberatung, Kurzzeitpflege). Lediglich ca. $60 \%$ der Angehörigen in der Treatmentgruppe nahmen eines der kostenlosen Angebote in Anspruch; die Angehörigen, die theoretisch alle Unterstützungsangebote wahrnehmen konnten, taten dies in der Praxis kaum. Somit konnten die erwarteten Unterschiede zwischen den Treatment-Gruppen nicht nachgewiesen werden. Ein bemerkenswertes Ergebnis zeigte sich jedoch hinsichtlich 
der Stabilität der häuslichen Pflegesituation: Es fand sich eine Interaktion zwischen der verwandtschaftlichen Beziehung zum Pflegebedürftigen und dem Treatment. Bei pflegenden Kindern in der Treatmentgruppe verhinderten oder verzögerten die Interventionsmaßnahmen erwartungsgemäß die Entscheidung für eine Heimunterbringung des Pflegebedürftigen. Pflegende Ehepartner in den Treatmentgruppen entschieden sich demgegenüber häufiger für eine Heimunterbringung als die Ehepartner in der Kontrollgruppe. Erklären lässt sich dieser Befund möglicherweise damit, dass die Ehepartner im Gegensatz zu den pflegenden Kindern in den meisten Fällen mit dem Pflegebedürftigen zusammenleben und somit einer insgesamt höheren Belastung ausgesetzt sind. Haben sie einmal Unterstützungsangebote in Anspruch genommen, wird ihnen der volle Umfang der Belastung eher bewusst, was die Entscheidung für eine Heimunterbringung erleichtert.

\section{4) Psychologische und soziale Hilfsangebote}

Von allen Interventionsprogrammen sind bisher die längerfristigen psychologischen und sozialen Hilfsangebote am besten untersucht worden. Diese umfassen psychoedukative Programme, Gesprächsgruppen und Selbsthilfegruppen für pflegende Angehörige sowie Einzelberatungen durch psychologisch geschulte Fachkräfte (vgl. im Überblick Cooke, McNally, Mulligan, Harrison \& Newman, 2001; Pusey \& Richards, 2001). Die meisten Evaluationsstudien psychosozialer Interventionsprogramme untersuchen Angehörige von Demenzpatienten; Programme für Angehörige von Schlaganfallbetroffenen sind bislang seltener evaluiert worden (Glass, Berkman, Hiltunen, Furie, Glymour, Fay \& Ware, 2004; Heuvel, Witte, Stewart, Schurre, Sanderman \& Jong, 2002; Robinson, Francis, James, Tindle, Greenwell \& Rodgers, 2005).

Methodisch sind die Evaluationsstudien in diesem Bereich von unterschiedlicher Güte. Bis vor einigen Jahren wurden als Evaluationskriterien häufig eher deskriptive und unstandardisierte Maße verwendet, z. B. die subjektive Zufriedenheit der Nutzer mit dem Angebot, Wissen in Bezug auf die Erkrankung des Pflegebedürftigen, Zufriedenheit mit sozialer Unterstützung, die Teilnahmefrequenz oder der klinische Eindruck der Anleiter der Intervention in Bezug auf die Gruppenprozesse. Zunehmend wurden dann jedoch auch standardisierte Instrumente zur Evaluation von psychosozialen Interventionsprogrammen herangezogen. Die standardisierten Maße 
umfassen vorwiegend Belastungsskalen und klinisch-psychiatrische Instrumente, zumeist Depressionsskalen (Haley, Bown \& Levine, 1987; Quayhagen \& Quayhagen, 1989; Smith, Forster \& Young, 2004; Zarit, Anthony \& Boutselis, 1987), sowie Skalen zum allgemeinem Wohlbefinden und Coping (Choi, Lavohn \& Christensen, 1983). Auch die Vermeidung bzw. Verzögerung der Institutionalisierung des Pflegebedürftigen wurde als Evaluationskriterium eingesetzt (Greene \& Monahan, 1989).

Neben der weit verbreiteten Verwendung unstandardisierter Messinstrumente besteht das größte Defizit der Evaluationsstudien in diesem Bereich ebenfalls darin, dass selten ein Längsschnitt-Kontrollgruppen-Design angewendet wird (Zarit, 1998; Zarit \& Leitsch 2001; Zarit, Stephens, Townsend, Greene \& Femia, 2003).

In einer Metaanalyse von Toseland und Rossiter (1989) wurden Ergebnisse aus 29 Interventionsstudien untersucht, in denen Angehörigenkurse und -gruppen mit edukativem Ansatz evaluiert worden waren. Die meisten Gruppen waren auf 6 bis 8 Treffen beschränkt. In allen Studien (25 der insgesamt 29 Studien), in denen die Zufriedenheit der Teilnehmer oder eine klinische Einschätzung der Angehörigen durch die Gruppenleiter erhoben wurden, zeigte sich eine positive Bilanz. Die Teilnehmer gaben durchweg einen hohen Grad an Zufriedenheit an und die Gruppenleiter beurteilten den Gruppenprozess ausnahmslos als effektiv. Dagegen waren die Befunde für Studien, in denen standardisierte Messinstrumente verwendet wurden, weniger überzeugend. In 14 der 19 Studien mit standardisierten Skalen zeigten sich keine Veränderungen in Bezug auf die untersuchten Variablen.

Beispielhaft sollen im Folgenden Studien dargestellt werden, in denen ein Interventionseffekt nachgewiesen werden konnte.

\section{Psychoedukative Seminare}

Zur Evaluation eines psychoedukativen Seminars für Familien von Schlaganfallpatienten erhoben Evans und Held (1984) unter anderem den Zuwachs an Wissen anhand eines Wissenstests. Neben einer signifikanten Zunahme des schlaganfallspezifischen Wissens fiel es $91 \%$ der Teilnehmer leichter, Fragen an das therapeutische Team oder die Ärzte zu richten. 
In einer Studie von Evans, Matlock, Bishop, Stranahan und Pederson (1988) wurde überprüft, welche Form der Edukation am effektivsten ist. Es wurden zwei Interventionsansätze mit einer Kontrollgruppe verglichen. Eine Treatmentgruppe erhielt im Rahmen eines edukativen Seminars Informationen zur Pflege und Unterstützung von Schlaganfallpatienten. Die zweite Treatmentgruppe konnte zusätzlich zum edukativen Seminar individuelle Beratung in Anspruch nehmen. Sowohl nach sechs Monaten als auch nach einem Jahr war in beiden Treatmentgruppen eine Zunahme an schlaganfallbezogenen Wissen zu verzeichnen. Außerdem hatten sich einige Aspekte des Pflegealltags verbessert. Einzelberatung zusätzlich zum edukativen Seminar erwies sich als effektiver als das Seminar allein.

Toseland, Rossiter und Labrecque (1989) befassten sich mit der Frage, ob professionell angeleitete Gruppen für Angehörige von Alzheimer-Kranken effektiver sind als durch Laien angeleitete. Die Ergebnisse zeigten, dass beide Gruppen im Vergleich mit einer Kontrollgruppe, die lediglich Entlastung durch Tagespflege erhielt, einen Nutzen in Bezug auf psychisches Wohlbefinden und soziale Unterstützung erlebten. Allerdings verzeichneten die Teilnehmer der professionell angeleiteten Gruppen die größte Verbesserung in Bezug auf die psychische Symptomatik während die Teilnehmer der Laiengruppen die größte Verbesserung in Bezug auf die Nutzung informeller Unterstützungsnetzwerke erzielte. Als Erklärung führten die Autoren an, dass bei der Analyse von Videoaufzeichnungen der Gruppensitzungen ein Unterschied in Bezug auf den Inhalt der Gruppensitzungen auffiel. Professionelle Leiter legten den Schwerpunkt auf die Vermittlung von Problemlösestrategien und führten die Treffen effektiver. Laien-Gruppenleiter förderten die Geselligkeit stärker und betonten die Bedeutung informeller sozialer Beziehungen.

Ziel einer methodisch sorgfältig konzipierten prospektiven Studie von Mittelman, Ferris, Shulman, Steinberg, und Levin (1996) war die Evaluation der Langzeitwirkung eines Beratungs- und Unterstützungsprogramms für Familien von Alzheimer-Kranken. Im Rahmen einer randomisierten, kontrollierten Untersuchung wurden 206 Partner von Alzheimer-Patienten auf zwei Gruppen aufgeteilt. Die Experimentalgruppe erhielt eine intensive psychoedukative Intervention und für den 
Notfall einen konstanten professionellen Ansprechpartner zugeteilt. Die Teilnehmer der Kontrollgruppe konnten telefonische Beratung in Anspruch nehmen, was jedoch kaum genutzt wurde. Es zeigte sich, dass das Risiko der Heimunterbringung für die Alzheimer-Kranken mit Pflegenden in der Treatmentgruppe um ein Drittel unter dem für Kranke von Partnern aus der Kontrollgruppe lag. Angehörige, die an der Intervention teilgenommen hatten, behielten die Kranken durchschnittlich 329 Tage länger in der häuslichen Umgebung. Die Intervention zeigte den stärksten Effekt bei leicht- und mittelgradig Demenzkranken.

Coon und Mitarbeiter verglichen zwei psychoedukative Trainings mit pflegenden Angehörigen von Demenzerkrankten zur Reduktion von Depressionen und Aggressionen. In beiden Interventionsgruppen zeigte sich im Vergleich zu einer Kontrollgruppe eine Abnahme depressiver Symptome und aggressiver Einstellungen. Außerdem erwiesen sich das Ausmaß an depressiven Symptomen und die Form des emotionalen Ausdrucks von Aggressionen bei den Angehörigen vor Teilnahme am Training als wesentliche Moderatoren für die relativen Effekte der Intervention (Coon, Thompson, Steffen, Sorocco \& Gallager-Thompson, 2003). Die Autoren folgerten daraus, dass entsprechende Schlüsselvariablen vor der Durchführung von Trainings erhoben werden sollten, um die Intervention besser an die jeweiligen Bedürfnisse der Angehörigen anzupassen.

Ein weiterer Aspekt, der bisher nur vereinzelt systematisch untersucht worden ist, ist der Effekt, den eine psychoedukative Intervention mit ihrer angestrebten Modifikation des Angehörigenverhaltens auf den Pflegebedürftigen hat. Haupt, Karger, Baumgärtner, Kuminoti, Jänner und Schneider (2000) gingen der Frage nach, ob sich durch eine dreimonatige, strukturierte Gruppenintervention mit pflegenden Angehörigen von Demenzkranken eine Verbesserung der Alltagsfähigkeit und eine Reduktion der Verhaltensbeeinträchtigungen bei den Kranken erzielen lassen. Ferner war von Interesse, welche unabhängigen Variablen diese Verbesserungen vorhersagen würden. Auch wenn die Studie eine nur geringe Fallzahl aufwies (14 Demenzkranke), erscheinen die Ergebnisse vielversprechend: Es konnten signifikante Verbesserungen von gedächtnisassoziierten Alltagsleistungen sowie eine signifikante Verbesserung von Unruhezuständen und Angst bei den Demenzkranken nachgewiesen werden. 


\section{Einzelberatungen durch geschulte Fachkräfte}

Mant, Carter, Wade und Winner (2000) untersuchten die Effekte von Einzelberatungen anhand von 267 Angehörigen, die zu Hause Beratung durch eine sozialpädagogische Fachkraft erhielten. Die Treatmentgruppe war im Vergleich zu einer Kontrollgruppe aktiver, schätzte ihre allgemeine Gesundheit besser ein, und zeigte Verbesserungen der psychischen Symptomatik.

Die Effektivität individueller Beratung von pflegenden Angehörigen durch Professionelle und Laien stand im Mittelpunkt einer Studie von Toseland und Smith (1990). Die individuelle Beratung umfasste in beiden Treatmentgruppen acht wöchentliche Sitzungen. Angehörige, die professionelle Beratung erhielten, zeigten im Vergleich zur Kontrollgruppe besseres subjektives Wohlbefinden, weniger psychiatrische Symptome (unterteilt in die Dimensionen Depression, Angst, Feindseligkeit und globale Symptome) sowie positive Veränderungen in Bezug auf kognitive, emotionale und verhaltensbezogene Faktoren. Auch Pflegepersonen, die Beratung durch Laien erhielten, zeigten im Vergleich zur Kontrollgruppe signifikante Veränderungen im letztgenannten Punkt. Allerdings konnte für diese Gruppe keine signifikante Zunahme im subjektiven Wohlbefinden oder in zwei Subskalen der psychiatrischen Symptome (Depression und Feindseligkeit) nachgewiesen werden. Weder individuelle Beratung durch Professionelle noch durch Laien hatte einen Effekt auf die Nutzung formaler sozialer Dienste.

Einen interessanten Interventionsansatz untersuchten Grant, Elliott, Weaver, Bartolucci und Giger (2002): Mit Angehörigen von Schlaganfallbetroffenen wurde ein Problemlösetraining per Telefon durchgeführt. Nach einem dreistündigen Hausbesuch eine Woche nach Entlassung aus einer Rehabilitationseinrichtung, in dem die pflegenden Angehörigen ( $\mathrm{N}=24)$ ein Problemlösetraining durch eine Fachpflegekraft erhielten, wurde das Training telefonisch fortgesetzt, zunächst vier Wochen lang jede Woche, danach weitere zwei Monate lang jeweils vierzehntägig. Im Vergleich mit der Placebogruppe und der Kontrollgruppe zeigten die Teilnehmer in der Treatmentgruppe verbesserte Problemlösefähigkeiten und weniger Depressionen. Außerdem ließen sich Verbesserungen in Bezug auf Vitalität, soziale Aktivitäten und Rolleneinschränkungen 
nachweisen. In Bezug auf das pflegebezogene Belastungsempfinden konnten jedoch keine signifikanten Unterschiede festgestellt werden.

\section{Zusammenfassung}

Gemessen an der Vielfalt der in den vergangenen Jahren entwickelten Interventionsprogramme, ist die methodische Güte vieler Evaluationsstudien immer noch als unzureichend zu beurteilen. Am besten untersucht sind bislang verschiedene psychoedukative Programme. Die vorliegenden empirischen Befunde zeigen, dass Interventionen, die sorgfältig entwickelt und implementiert wurden, pflegenden Angehörigen durchaus nutzen. Allerdings lassen sich die positiven Auswirkungen in den meisten Studien nur bedingt durch standardisierte Maße nachweisen.

Ein wesentliches Problem bei der Implementierung von Unterstützungsangeboten stellt die mangelhafte Nutzung der Programme dar, was auf verschiedene Gründe zurückzuführen ist. Dazu zählen auf die Pflegeperson bezogene Hindernisse (zu hohe Kosten, sozialer Druck, Schuldgefühle gegenüber dem Angehörigen), auf den Pflegebedürftigen bezogene Hindernisse (die Symptomatik des Patienten, z. B. Immobilität, lässt eine Nutzung nicht zu) sowie strukturelle und institutionelle Hindernisse (räumliche Entfernung, Inflexibilität der Entlastungsangebote, lange Wartezeiten, mangelnde Kenntnis von Angeboten).

\subsubsection{Methodische Implikationen für die vorliegende Studie}

Auf der Grundlage der in den vorigen Abschnitten vorgestellten Evaluationsstudien und der kritischen Überlegungen in Bezug auf methodische Aspekte werden im Folgenden methodische Implikationen für die vorliegende Arbeit formuliert.

\section{Forschungsdesign}

Bei der Evaluation von Interventionsmaßnahmen erfüllen Forschungsdesigns in vielen Fällen nicht die methodischen Standards (Cooke, McNally, Mulligan, Harrison \& Newman, 2001; Pusey \& Richards, 2001; Zarit \& Leitsch, 2001). Das gravierendste Problem besteht darin, dass es häufig keine unbehandelte Kontrollgruppe gibt. Auch 
werden in vielen Fällen lediglich Querschnitt- statt Längsschnitt-Daten erhoben. Die Pflegesituation konstituiert jedoch einen dynamischen Prozess, dem eine Längsschnittuntersuchung mit mehreren Messzeitpunkten am ehesten angemessen ist.

Dabei ist der Vergleich einer Experimental- mit einer Kontrollgruppe im zeitlichen Verlauf von großer Bedeutung, da sich auch ohne Intervention Befindlichkeits- und Belastungsmaße über die Zeit signifikant verändern. Ohne unbehandelte Kontrollgruppe wären somit positive Veränderungen nicht ohne weiteres als Interventionseffekte $\mathrm{zu}$ deuten. Allerdings ist $\mathrm{zu}$ berücksichtigen, dass eine „unbehandelte“ Kontrollgruppe in den meisten Fällen nur in Bezug auf die zu evaluierende Intervention unbehandelt ist. Denn viele pflegende Angehörige nehmen im Laufe des Pflegeprozesses irgendeine Form der Unterstützung in Anspruch (Knight, Lutzky \& Macofsky-Urban, 1993). In welchem Umfang dies geschieht, sollte in Interventionsstudien unbedingt systematisch erhoben werden.

Ein weiteres Problem der Forschungsdesigns in Evaluationsstudien ist die Verwendung relativ kleiner Stichproben; so stützen einige Arbeiten ihre Aussagen lediglich auf die Evaluation einer einzelnen Angehörigengruppe mit 9 Teilnehmern (Wilz, Gunzelmann, Adler \& Brähler, 1998).

\section{Auswahl der Messinstrumente}

Drei Aspekte sollten nach Zarit und Leitsch (2001) bei der Wahl der Messinstrumente berücksichtigt werden:

1. die Verwendung von Messinstrumenten, die anhand einer allgemeinen Population standardisiert oder die spezifisch für pflegende Angehörige entwickelt wurden;

2. die Veränderungssensitivität der Messinstrumente;

3. die Wahl eines quantitativen oder eines qualitativen Ansatzes.

George (1994) postuliert, dass die Pflegeforschung standardisierte Messinstrumente benutzen sollte, die für nicht-pflegende Populationen entwickelt wurden. Die Autorin argumentiert, dass nur im Vergleich mit nicht-pflegenden Populationen die negativen Effekte der Pflege herausgestellt werden könnten und somit 
politische Entscheidungsträger für die Implementierung weiterer Entlastungsmöglichkeiten für Angehörige zu gewinnen sind. Messinstrumente, die spezifisch für pflegende Angehörige entwickelt wurden, haben dagegen meist unzureichende psychometrische Eigenschaften und sind aufgrund der fehlenden Referenzgruppe schwer zu interpretieren. Normative Messinstrumente haben jedoch andererseits den Nachteil, dass sie unter Umständen nicht adäquat die Prozesse erfassen, die sich als Konsequenz der Intervention verändern, da sie entwickelt wurden, um ein bestimmtes Phänomen in der allgemeinen Bevölkerung zu messen. Der dritte von Zarit und Leitsch (2001) diskutierte Aspekt tangiert die Frage, ob quantitative oder qualitative Methoden zum Einsatz kommen. In einigen Interventionsstudien wird lediglich ein qualitativer Ansatz verwandt. Als alleinige Messmethode ist dies jedoch nicht ausreichend, da die Interpretation der Befunde zu subjektiv sein kann. Außerdem stellt sich bei rein qualitativen Studien die Schwierigkeit, Ergebnisse verschiedener Untersuchungen miteinander zu vergleichen. Ein optimaler Ansatz ist die Kombination quantitativer mit qualitativen Methoden, was in der vorliegenden Studie verwirklicht wurde.

\section{Abgestufte Evaluation}

Zarit und Leitsch (2001) weisen auf die Bedeutung einer abgestuften Evaluation hin. Zunächst sollte im Sinne einer Prozess-Evaluation festgestellt werden, ob die unmittelbaren Ziele der Intervention tatsächlich erfüllt wurden (z. B. ob es gelang, den Informiertheitsgrad oder die soziale Unterstützung zu erhöhen), bevor andere Maße (wie Depressionen oder Angst) erhoben werden. Andernfalls sei es nicht möglich zu ermitteln, ob negative Ergebnisse darauf zurückzuführen seien, dass die Intervention nicht effektiv war, oder darauf, dass die Intervention lediglich nicht adäquat implementiert wurde.

Die vorstehend benannten methodischen Implikationen werden in Kapitel 3.1 bei der Eingrenzung der Forschungsfragen und Formulierung der Hypothesen nochmals aufgegriffen. Im darauf folgenden Kapitel wird das untersuchte Interventionsprogramm ausführlich beschrieben. 


\section{Darstellung des untersuchten Interventionsprogrammes und Forschungsfragen}

\subsection{Eingrenzung des Untersuchungsgegenstandes - Fragestellungen und Hypothesen}

Wie bereits in Kapitel 2.8.4 erwähnt, sind bislang vor allem Interventionen für Angehörige von Demenzkranken untersucht worden (Glosser \& Wexler, 1985; Walker, Pomeroy, McNeil, \& Franklin, 1994). Nur wenige Studien befassen sich mit Interventionsangeboten für Angehörige von Schlaganfallpatienten. Die Situation dieser Angehörigen unterscheidet sich zweifellos von der Situation derjenigen, die einen Demenzkranken betreuen. Bei Schlaganfallpatienten schließt sich an die Akutversorgung in vielen Fällen eine Rehabilitation an, bei der es sehr wichtig ist, dass der Umgang der Angehörigen mit dem Schlaganfallpatienten mit den Bemühungen des Rehabilitationsteams synchronisiert wird. Es bestehen bei Schlaganfallpatienten zum Teil Mobilitätseinschränkungen, die eine stärkere Anpassung der Lebensumwelt erforderlich machen, als dies bei Demenzpatienten der Fall ist. Darüber hinaus müssen Angehörige auch hinsichtlich der Umsetzung der Sekundärprophylaxe eingebunden werden und Informationen über eine eventuell notwendige Umstellung der Lebensweise erhalten (fettreduzierte, vitaminreiche Ernährung, Raucherentwöhnung, regelmäßig körperliche Betätigung). Außerdem werden eventuell vorhandene neuropsychologische Defizite und Persönlichkeitsveränderungen häufig zunächst nicht adäquat von den Angehörigen eingeordnet, da die körperlichen Defizite zumeist augenfälliger sind. Dies erfordert Aufklärungsbedarf. Neben der Vermittlung von Wissen zum adäquaten Umgang mit dem Pflegebedürftigen nimmt gleichermaßen die Unterweisung in hilfreichen Strategien zum Umgang mit pflegebedingter Belastung eine zentrale Stellung ein.

Im Rahmen der Studie wurde ein psychoedukativer Kurs für Angehörige von Schlaganfallpatienten entwickelt, der die oben genannten Aspekte entsprechend berücksichtigt. Zentrale Fragestellung der begleitenden Evaluationsstudie war, ob die Teilnehmer von der Intervention profitieren. 
Bevor die Forschungsfragen weiter ausdifferenziert und Hypothesen formuliert werden, veranschaulicht Abbildung 4 anhand des bereits in Kapitel 2.5.2 vorgestellten Stressbewältigungsmodells (nach Kramer, 1993) die postulierte Wirkweise des untersuchten psychoedukativen Kurses.
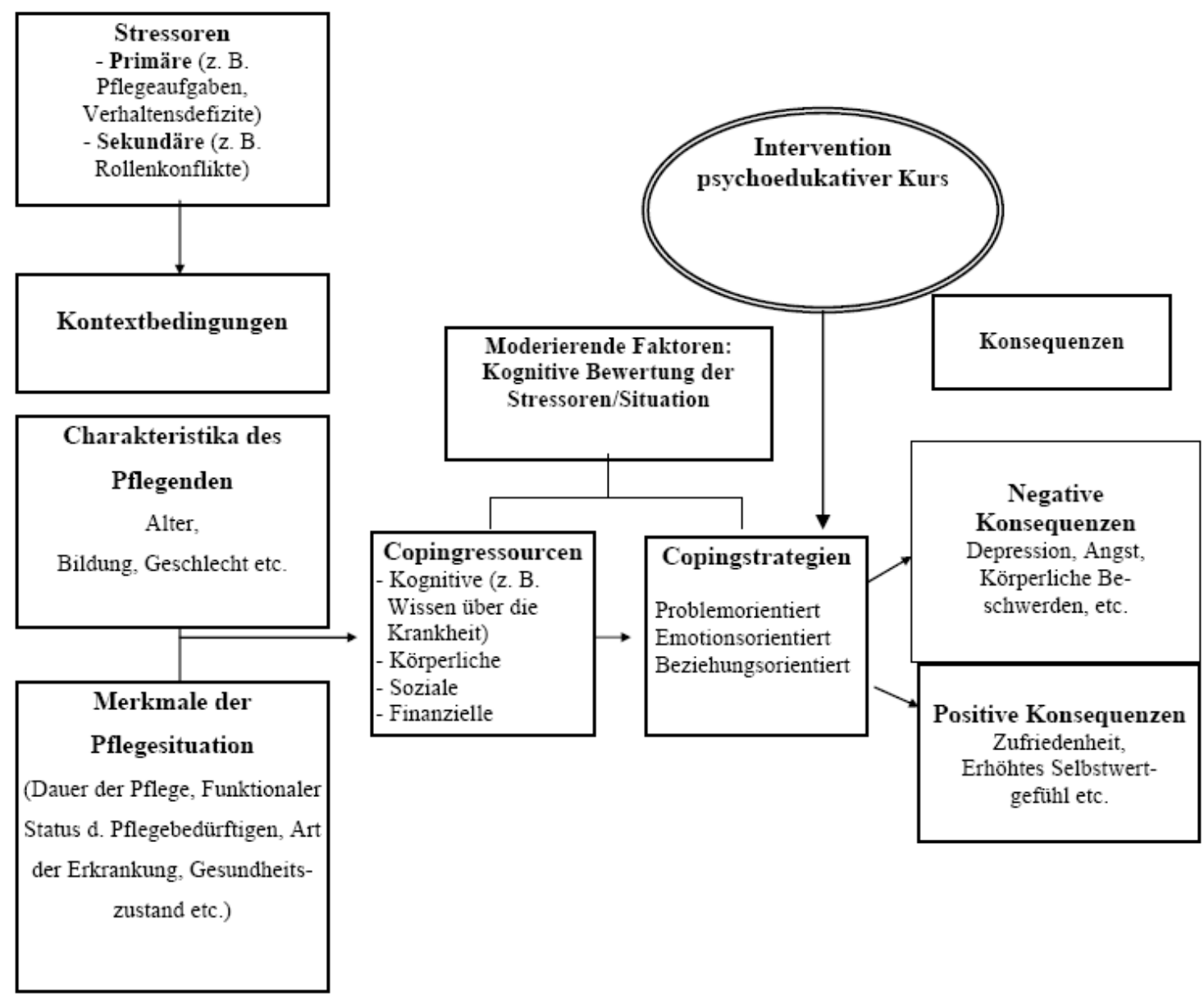

Abbildung 4: Wirkweise des psychoedukativen Kurses im Rahmen des Stressbewältigungsmodells (nach Kramer, 1993)

Für die Intervention Angehörigenkurs wird ein Effekt auf Indikatoren subjektiver Befindlichkeit wie Depressivität, Ängstlichkeit, körperliche Beschwerden angenommen, der indirekt über die Verbesserung der Bewältigungsstrategien wirksam wird. Dementsprechend standen die Variablen „Copingstrategien“ und „Konsequenzen“ im Fokus des Interesses. 
Aus der in Abbildung 4 postulierten Wirkweise des Angehörigenkurses und der bestehenden Forschungsliteratur wurden folgende Hypothesen in Bezug auf die Effekte der untersuchten Intervention abgeleitet:

1. Ein Übersichtsartikel von Schulz, O’Brien, Czaja und Mitarbeiter (2002), der seit 1996 publizierte Evaluationsstudien für Angehörige von Demenzkranken umfasst, zeigt, dass sich die emotionale Befindlichkeit (Depression und Angst) für Teilnehmer an psychoedukativen Interventionen in 24 der 34 untersuchten Studien verbesserte. Entsprechend wird für das Kursprogramm für Angehörige von Schlaganfallpatienten angenommen, dass sich die emotionale Befindlichkeit (Depression und Angst) der teilnehmenden Angehörigen im Gegensatz zu den Nicht-Teilnehmern im zeitlichen Verlauf verbessert (Hypothese 1).

2. Auch für körperliche Beschwerden der pflegenden Angehörigen liess sich in einigen Studien nachweisen, dass diese durch eine psychoedukative Intervention verringert werden konnten (Schulz, O’Brien, Czaja et al., 2002). Es wird daher angenommen, dass sich die Kursteilnahme ebenfalls günstig auf die körperlichen Beschwerden der Angehörigen von Schlaganfallpatienten auswirkt (Hypothese 2).

3. In Evaluationsstudien wird selten der Zuwachs an Wissen erhoben (Pusey \& Richards, 2001). Dies wird vielfach kritisiert (Zarit \& Leitsch, 2001), da eine notwendige Bedingung für die Realisierung der distalen Ziele einer Intervention (z. B. Veränderung der psychischen Befindlichkeit, Verbesserung der CopingStrategien) darin besteht, dass die unmittelbaren Ziele der Intervention (z. B. Wissenszuwachs) erreicht wurden. Für die vorliegende Evaluationsstudie wird angenommen, dass sich bei den Teilnehmern am Kurs im Gegensatz zu den Nicht-Teilnehmern eine Zunahme des schlaganfallspezifischen Wissens im zeitlichen Verlauf feststellen lässt (Hypothese 3).

4. In Bezug auf die Frage nach einem Zusammenhang zwischen Copingstrategien, Kursteilnahme und Indikatoren emotionalen und körperlichen Wohlbefindens gibt es keine konkreten Hypothesen. Vielmehr soll ein möglicher Zusammenhang explorativ anhand einer Teilstichprobe untersucht werden. Wie schon ausführlich in Kapitel 2.6.4 dargestellt, fand Lämmler (1998) in seiner Studie zwei Bewältigungsstile, die sich im Ausmaß der Patientenfixierung und 
der Flexibilität, auf veränderte Bedingungen in der Pflege $\mathrm{zu}$ reagieren, voneinander unterscheiden. Aus den methodischen Implikationen für die Studie (Kapitel 2.8.5) geht hervor, dass eine Integration inferenzstatistischer und qualitativ-deskriptiver Befunde den Erkenntnisgewinn in Bezug auf die Evaluation von Interventionseffekten fördern kann. Dementsprechend fand auch im Rahmen der vorliegenden Untersuchung zur Beantwortung dieser Fragestellung eine Integration der methodischen Herangehensweisen statt. Neben der statistischen Prüfung von Hypothesen, die aus der bestehenden Forschungsliteratur entwickelt wurden, werden vertiefend die verwendeten Copingstrategien anhand einer qualitativen Methodik analysiert, um so ein besseres Verständnis der zugrunde liegenden Mechanismen zu gewinnen. Da es gerade beim Coping um komplexe Prozesse mit dynamischer Entwicklung geht, erschien der Einsatz einer qualitativen Methodik angemessen. Bei der Tiefenanalyse sollten auch neue Aspekte aufgedeckt werden, die sich einer quantitativen Untersuchung weitgehend entziehen. 


\subsection{Das untersuchte Interventionsprogramm: Psychoedukativer Kurs für pflegende Angehörige von Schlaganfallbetroffenen}

Die vorliegende Dissertation entstand aus einem Forschungsprojekt zur Intensivierung der Angehörigenarbeit nach einem Schlaganfall, das von der Robert-Bosch-Stiftung gefördert wurde. Die Studie wurde im Zeitraum von Januar 1996 bis Mai 1999 an der Tagesklinik des Evangelischen Geriatriezentrums Berlin durchgeführt, in der schwerpunktmäßig Schlaganfallpatienten behandelt werden. Im Rahmen des Projektes wurde ein abgestuftes Konzept der Angehörigenarbeit entwickelt (Heier, 2000), das aus mehreren Elementen besteht, wobei der psychoedukative Kurs für Angehörige von Schlaganfallpatienten der zentrale Baustein ist.

Als Zielsetzungen für den Kurs wurden angestrebt:

- Angehörige umfassend über die Erkrankung und die Auswirkungen zu informieren und damit ihre Kompetenzen im Umgang mit dem Betroffenen zu erhöhen;

- Pflegenden die Möglichkeit zu bieten, Erfahrungen und Probleme mit anderen auszutauschen, wodurch sie eine Bestätigung ihrer Situation erleben und emotional entlastet werden;

- Angehörigen das Konzept der "Selbstpflege" nahe zu bringen und Stressbewältigungsstrategien zu vermitteln;

- Raum für gegenseitige emotionale Unterstützung zu bieten.

Der Angehörigenkurs umfasste 10 Termine und fand einmal wöchentlich statt. An jedem Termin wurde ein Themenschwerpunkt von einem Mitarbeiter des therapeutischen Teams behandelt. Bei der Gestaltung der Kursabende wurde darauf Wert gelegt, dass neben der Wissensvermittlung jeweils ausreichend Zeit blieb, damit die Teilnehmer ihre eigenen Erfahrungen zum Thema austauschen und Fragen stellen konnten (zu Hinweisen zur Kurs- und Seminargestaltung siehe auch Knoll, 1993 und Müller, 1989). $\mathrm{Zu}$ jedem Themenschwerpunkt erhielten die Teilnehmer schriftliche Materialien und weiterführende Literaturhinweise. Eine kontinuierliche Begleitung des Kurses fand durch die Autorin statt, die die Moderation und Gesprächsführung übernahm. Die Gruppengröße lag zwischen 10 und 14 Teilnehmern. Der Kurs umfasste folgende Themen: 
1. Einführungsveranstaltung

2. Medizinische Ursachen des Schlaganfalls, Verlauf und Behandlung

3. Aktivierende Pflege, Pflegetechniken und Prophylaxe

4. Hirnleistungsstörungen nach Schlaganfall und ihre Behandlung

5. Entlastungsmöglichkeiten im Pflegealltag/Leistungsspektrum und materielle Hilfen

6. Prinzipien von Transfer, Lagerung und rückengerechtem Arbeiten, BobathKonzept

7. Alltagsaktivitäten und der Einsatz von Hilfsmitteln als Hilfe zur Selbsthilfe

8. Umgang mit Sprach- und Sprechstörungen

9. Umgang mit Belastung und Stress im Pflegealltag

10. Abschlussveranstaltung

\section{Inhalte des Angehörigenkurses}

Die folgende stichpunktartige Zusammenfassung der Kursinhalte soll einen Überblick über die behandelten Aspekte bieten:

\section{Einführungsveranstaltung}

Kursleitung: Diplom-Psychologin

- Organisatorisches

- Erläuterung der Kursziele

- Vorstellungsrunde der Teilnehmer

- Leitfragen:

- Name, Alter etc.

- derzeitige Lebenssituation (Größe des Haushalts, Verwandtschaftsverhältnis zum Pflegebedürftigen, Berufstätigkeit etc.)

- Dauer der Pflege

- Erwartungen an den Kurs

- Sammlung von speziellen Wünschen bezüglich der Kursthemen

- Motive zur Übernahme von Pflege 
II. Schlaganfall - Ursachen, Verlauf und Behandlung

Referentin: Ärztin

- Epidemiologie

- Ursachen des Schlaganfalls

- Risikofaktoren

- Vorbeugende Maßnahmen

- Warnzeichen und Vorboten eines Schlaganfalls

- Verhalten bei einem akuten Schlaganfall

- Medikamentöse Behandlung

- Hinweise zur Ernährung

- Rehabilitationsmaßnahmen

- Ängste, Befürchtungen bezüglich eines erneuten Schlaganfalls und Umgang damit

III. Aktivierende Pflege, Pflegetechniken und Prophylaxe

Referentin: Pflegefachkraft

- aktivierende Pflege

- richtige Ernährung

- Inkontinenzprobleme, psychische Probleme im Umgang mit Inkontinenz

- Inkontinenzhilfsmittel, Kontinenztraining

- Dekubitusprophylaxe

- Hinweise zur Medikamenteneinnahme

- Waschen und Baden

- Kriterien für die Auswahl ambulanter Pflegedienste 
IV. Hirnleistungsstörungen nach Schlaganfall und ihre Behandlung

Referentin: Neuropsychologin

- Neuropsychologische Einschränkungen im Bereich von

- Aufmerksamkeit

- Gedächtnis

- Wahrnehmung

- Denken

- Stimmung und Verhalten

- Relevanz der neuropsychologischen Einschränkungen für den Alltag

- Therapie von Hirnleistungsstörungen

- Förderung hirnleistungsgestörter Patienten zu Hause

- Veränderung der Beziehung zum Patienten

- Umgang mit persönlichkeitsveränderten Pflegebedürftigen

$\underline{\text { V. Entlastungsmöglichkeiten im Pflegealltag/Leistungsspektrum und materielle Hilfen }}$ Referentin: Dipl.-Sozialarbeiterin

- Möglichkeiten der Pflegeentlastung durch Inanspruchnahme/Vernetzung von Pflegeund Hilfsdiensten am Beispiel von:

- Sozialstationen

- mobilen Diensten (Schiebedienste/Besuchsdienste/Handwerkerdienste)

- Kurzzeit- bzw. Urlaubspflege

- Beratungsstellen (Entlastungsgespräche/Pflegeplanung)

- Materielle Hilfen

- Leistungen aus der Pflegeversicherung

- Hilfsmittelversorgung bzw. Hilfsmittelverleih

- Reflexion der Gründe für Nicht-Inanspruchnahme von entlastenden Maßnahmen ("man muss es alleine schaffen" etc.) 
VI. Prinzipien von Transfer, Lagerung und rückengerechtem Arbeiten, Bobath-Konzept Referentin: Physiotherapeutin

- Darstellung des Bobath-Konzepts und der wichtigsten Prinzipien der BobathTherapie

- Hinweise zu rückenschonendem Arbeiten

- Demonstration des Transfers vom (Roll-)Stuhl ins Bett

- Demonstration korrekter Lagerung im Bett

- Demonstration der Lagerung am Tisch

- Demonstration der Schultermobilisation und Erklärung der Gefahren bei unsachgemäßem Umgang mit einer subluxierten Schulter

- Möglichkeit zum Üben der Teilnehmer

- Empfehlungen für die Unterstützung der Therapie zu Hause und Erläuterung der Gefahren falschen Handlings

- ambulante Physiotherapie

- legitime Forderungen vs. überhöhte Ansprüche/Erwartungen an den Pflegebedürftigen (speziell in Bezug auf Laufen, Treppensteigen etc.)

VII. Alltagsaktivitäten und der Einsatz von Hilfsmitteln als Hilfe zur Selbsthilfe Referentin: Ergotherapeutin

- Was ist Ergotherapie?

- Maßnahmen zur Wohnungsanpassung (behindertengerechte Umbaumaßnahmen)

- Förderung der Aktivitäten des täglichen Lebens

- Waschen und Anziehen

- Hausarbeiten

- Benutzung von Verkehrsmitteln

- Einkaufen

- Demonstration von Hilfsmitteln

- Allgemeines

- Mobilitätshilfen

- Wasch- und Anziehhilfen 
- Hilfen im Sanitätsbereich

- Hilfen im Küchenbereich

- Gefahren der Überversorgung und überprotektiven Verhaltens

- ambulante Ergotherapie

- legitime Forderungen vs. überhöhte Ansprüche/Erwartungen an den

Pflegebedürftigen (speziell in Bezug auf die Aktivitäten des täglichen Lebens)

VIII. Wie finden wir eine gemeinsame Sprache? - Der Umgang mit Aphasikern

Referentin: Sprachtherapeutin

- Bedeutung von Sprache im mitmenschlichen Umgang

- Erklärung der zwei Formen einer Sprachstörung nach Schlaganfall

- Aphasie

- Dysarthrie

- Kurzer Überblick über die Formen der Aphasie (Aphasiesyndrome: Globale Aphasie, Wernicke-Aphasie, Broca-Aphasie, Amnestische Aphasie)

- Modell der Sprachverarbeitung

- Beispiele für Sprachfehler (auf Lautebene, Wortebene, Satzebene, Sprachautomatismen)

- Hinweise zum Umgang mit Aphasikern

- Wie erleichtert man dem Aphasiker, seine Gedanken zu übermitteln?

- Wie erleichtert man dem Aphasiker das Verständnis?

- Therapie der Aphasie

- Ziel: Wiederherstellen der Sprache bzw. Verbesserung der Kommunikationsfähigkeit

- Methoden (Beispiele für Übungen) 
IX. Umgang mit Belastung und Stress im Pflegealltag

Referentin: Dipl.-Psychologin

- Erfahrungsaustausch über Belastungen in der Pflegesituation:

- Veränderungen der eigenen Lebensplanung

- Angebundensein, ausschließliche Zuständigkeit

- veränderte Rollen

- Kommunikationsprobleme

- Wesensveränderungen des Pflegebedürftigen

- körperliche Belastung

- fehlende Anerkennung

- soziale Isolation

- Konflikte mit anderen Familienmitgliedern

- finanzielle Probleme

- kognitive, emotionale und körperliche Überforderungsreaktionen bei Stress

- Ansatzpunkte zur Stressbewältigung, adäquate und schädliche Stressbewältigungsstrategien

- Ermutigung zur "Selbstpflege"

- Umgang mit Schuldgefühlen

\section{$\underline{X . \text { Abschließende Diskussionsrunde }}$}

Leitung: Dipl.-Psychologin

- Feedback der Teilnehmer zum Kurs

- Reflexion der Rolle als Pflegender

Leitfragen:

- Was für Ratschläge würden Sie jemandem geben, der gerade pflegender Angehöriger geworden ist?

- Würden Sie heute, nach allem, was Sie jetzt wissen, noch einmal die Pflege Ihres Angehörigen übernehmen? Oder würden Sie heute anders entscheiden?

- Ermutigung zur Selbstpflege

- Hinweis auf die Möglichkeit der Einrichtung einer Gesprächgruppe 


\section{Methode}

In der vorliegenden Arbeit wurden unterschiedliche methodische Zugänge zum Forschungsthema miteinander kombiniert. Neben quantitativen kamen auch qualitative Verfahren zum Einsatz. Bevor die jeweiligen methodischen Vorgehensweisen in den Kapiteln 5 und 7 erläutert werden, liefert der folgende Abschnitt zunächst einen Überblick über methodologische und wissenschaftstheoretische Aspekte. Des Weiteren wird eine Standortbestimmung der vorliegenden Arbeit vorgenommen und Implikationen für die Methodenwahl diskutiert. Kapitel 5 gibt dann einen Überblick über das Forschungsdesign des quantitativen Teils der Erhebung, wobei die Stichprobe, der Untersuchungsablauf und die Erhebungsinstrumente eingehend beschrieben werden. Es folgt eine Ergebnisdarstellung des quantitativen Teils in Kapitel 6. Kapitel 7 behandelt die Methodik des qualitativen Teils der Untersuchung mit einer Darstellung der Stichprobenauswahl, des Interviewertrainings sowie der Erhebungs- und Auswertungsmethoden der Interviews. Daran schließt sich die Darstellung der Ergebnisse des qualitativen Teils in Kapitel 8 an.

\subsection{Wissenschaftstheoretische Aspekte quantitativer und qualitativer Methoden} Qualitativen und quantitativen Methoden werden - besonders in Deutschland fundamental inkompatible Denkweisen und Weltsichten zugeschrieben (Kelle \& Erzberger, 2003). Bei näherer Betrachtung entpuppen sich die Abgrenzungen jedoch durchaus als überwindbar, wie in den nächsten Abschnitten gezeigt werden soll. In der Psychologie und Soziologie überwiegen zwar nach wie vor aufgrund der positivistischen Denktradition die quantitativen „Puristen“, die bei ihren Erhebungsmethoden und Auswertungsverfahren das Schwergewicht auf die Quantifizierung bzw. Auszählung von zu beobachtenden Merkmalen und Einheiten legen. Doch ist trotz der allgemeinen Vorherrschaft statistischer Auswertungs- und Beweisverfahren in den letzten Jahren ein allmähliches Aufbrechen der bislang starren wissenschaftstheoretischen Positionen zu beobachten. In zunehmendem Maße gewinnen qualitativ-interpretative Verfahren wieder an Bedeutung. Dies dürfte auch durch eine Krise in der quantitativen Forschung beschleunigt worden sein, die mit der Erkenntnis 
einherging, dass die genaue Erfassung komplexer Einzelfälle vernachlässigt worden ist (Lämmler, 1998). Parallel zu der Hinwendung zu rein qualitativen Verfahren, wird heute zunehmend auch eine Integration quantitativer und qualitativer Ansätze in der Forschung postuliert. Je nach wissenschaftstheoretischer Forschungstradition wird dabei jedoch den jeweiligen methodischen Herangehensweisen ein unterschiedlicher Stellenwert beigemessen. Nach Garz und Kraimer (1991) lassen sich diesbezüglich insgesamt drei methodologische Positionen unterscheiden: der Vorrang quantitativer Forschungsansätze, der Vorrang qualitativer Forschungsansätze und die Gleichberechtigung quantitativer und qualitativer Ansätze. Diese sollen im Folgenden kurz umrissen werden.

\section{Vorrang der quantitativen Forschungsansätze unter Einbezug qualitativer Anteile}

Qualitative Ansätze werden allenfalls in der Anfangsphase eines Forschungsunternehmens zur Exploration und Hypothesengenerierung akzeptiert (Miller, 1986). Allerdings stellt die rein qualitative Verarbeitung von derart gewonnener Information nach Thomae (1984) keine Alternative zur quantitativen Herangehensweise dar. Für die wissenschaftliche Überprüfung der Forschungshypothesen sind allein quantitativ-statistische Methoden vorgesehen. Dabei wird häufig die Repräsentativität der Stichprobe als Begründung dafür angeführt, dass erst die quantitativen Daten zu Ergebnissen im eigentlichen Sinne führen. Grundsätzlich wird qualitative Forschung zwar als hilfreiche, ,illustrative“ Ergänzung, prinzipiell aber als verzichtbar angesehen, sodass sich diese Position bei näherer Betrachtung als „,verdeckte Version des quantitativen Purismus“ (Garz und Kraimer, 1991, S. 15) erweist.

\section{Vorrang der qualitativen Forschungsansätze unter Einbezug quantitativer Anteile}

Diese Position wird seltener, dafür aber umso radikaler vertreten. Daten, die in standardisierten, quantitativen Verfahren erhoben wurden, werden lediglich als Indiz für die zugrunde liegenden Prozesse und Zusammenhänge zugelassen. An die eigentlichen Phänomene hofft man dagegen mit

„...Beobachtungsverfahren und Fallstudien heranzukommen, die die Handlungsabläufe in ihrem situativen Kontext so weit wie möglich zu erhalten 
versuchen. Bevorzugt werden Vorgehensweisen, die vermeiden, Teilaspekte voreilig $\mathrm{zu}$ isolieren, und die sich bemühen, die beobachteten Vorgänge nicht unter Gesichtspunkten vorab definierter Konstrukte und Hypothesen analytisch zu zergliedern sondern alle Anstrengung darauf verwenden, einzelne Prozesse und einzelne Fälle bis ins Detail zu erfassen“ (Krappmann, Oevermann \& Kreppner, 1974, S. 10, zitiert in Heinze, 1987).

Eine stringente Analyse eines biographischen Einzelfalles könne mitunter mehr an Beweiskraft bieten als das, was auf der Grundlage einer „repräsentativen Stichprobe“ ermittelt werde (Heinze, 1987). Darüber hinaus wird quantitativen Methoden ein stärkerer Abstraktionsgrad zugeschrieben als qualitativen Verfahren. Kleining (1982) argumentiert dementsprechend, dass qualitative Forschung in der Forschungspraxis quantitativer Forschung vorausgehen sollte. Dabei betont er, dass ein qualitativer Ansatz jedoch nicht in jedem Fall von quantitativer Forschung ergänzt werden müsse. Denn nach Kleining (1982) liefere eine Quantifizierung keinen Erkenntnisgewinn, wenn ein qualitativer Zugang den Forschungsgegenstand bereits erkläre. Kann die Forschungsfrage nicht befriedigend durch qualitative Forschung geklärt werden, so könne ein quantitativer Ansatz die Erkenntnislücke auch nicht schließen. Quantitative Forschung wird somit als ,zweitbeste Lösung' und als prinzipiell verzichtbar angesehen.

\section{Gleichberechtigung quantitativer und qualitativer Forschungsansätze}

In Abgrenzung $\mathrm{zu}$ den oben dargestellten, in der Essenz puristischen Positionen, postulieren verschiedene Autoren (z. B. Fromm, 1990) eine Methodenkombination, wobei die Anwendung einer bestimmten Methode von den Merkmalen des Forschungsgegenstandes und nicht vom eigenen wissenschaftstheoretischen Standpunkt abhängig gemacht werden sollte (Wilson, 1982). Durch die Verbindung diverser Methoden soll der Forschungsgegenstand aus unterschiedlichen Perspektiven auf unterschiedliche Weise betrachtet werden, was zu einem umfassenderen und valideren Gesamtbild führen soll. Dieses Vorgehen wird oft als Triangulation bezeichnet (Denzin, 1989). 
Dass bislang qualitative und quantitative Daten jedoch eher selten auf diese Art und Weise in Studien kombiniert werden, ist neben erkenntnistheoretischen Aspekten auch auf praktische Gründe zurückzuführen (Rank, 1992). Dazu gehören, dass die Anwendung verschiedener Methoden bei der Bearbeitung eines Themas naturgemäß auch die Kosten des Forschungsprojekts erhöht. Des Weiteren lassen sich Studien mit unterschiedlichen Forschungszugängen oft schwerer veröffentlichen, da Gutachter, die entweder die eine oder andere wissenschaftstheoretische Position favorisieren, den jeweils anderen Forschungsansatz möglicherweise kritisch beurteilen. Außerdem gibt es in der universitären Ausbildung eine starke Tendenz hin $\mathrm{zu}$ den quantitativen Forschungsansätzen. Somit fehlen vielen Wissenschaftlern Kenntnisse und Fertigkeiten in Bezug auf qualitative Methoden.

Die Integration beider Forschungsansätze birgt laut Rank (1992) jedoch verschiedene Vorteile. Ein wesentlicher Vorteil ist die Komplementarität der jeweiligen Forschungsrichtungen. So lassen sich durch quantitative Daten Informationen über Regelhaftigkeiten und Häufigkeiten bestimmter Variablen gewinnen sowie Aussagen über allgemeine Modelle treffen, während qualitative Ansätze die Prozesse, die die Variablen miteinander verbinden, erhellen können (von Kardorff, 1991). Die Stärke qualitativer Daten liegt in ihrer Reichhaltigkeit und Tiefe, da qualitative Erhebungsmethoden eine Fülle von Informationen produzieren. Darüber hinaus erlaubt ein qualitativer Ansatz den Teilnehmern, ihre Erfahrungswelt so zu strukturieren, wie sie sie sehen, und nicht, wie der Wissenschaftler sie sieht. Dem Forschenden wird damit ein tieferes Verständnis des Forschungsgegenstandes ermöglicht. Demgegenüber liegt die grundsätzliche Stärke quantitativer Daten in der Reliabilität und Generalisierbarkeit der Ergebnisse.

Diese Position wirkt zunächst überzeugend - lässt sie doch die Hoffnung aufkommen, durch die Kombination verschiedenartiger Forschungsmethoden deren jeweilige Vorteile voll nutzen und ihre Nachteile ausgleichen zu können. Dennoch ist diese Ansicht nicht ohne Kritik geblieben (vgl. Flick, 1990). So bezweifeln Kritiker des integrativen Ansatzes die Möglichkeit eines Ausgleichs der Limitationen eines Forschungsansatzes durch einen zweiten Forschungsansatz. Sowohl quantitativ wie 
qualitativ orientierte Puristen unterstellen, dass quantitative und qualitative Forschungsmethoden keine identischen Ergebnisse hervorbringen könnten, da die Betrachtung des Forschungsgegenstandes aus einem völlig unterschiedlichen Blickwinkel erfolge. Die „Sprachspiele“ seien nicht aufeinander rückführbar und es gäbe keine Kriterien, um das eine Paradigma in ein anderes zu überführen. Aufgrund eines fehlenden geteilten Weltbildes sei nicht einmal eine Abgleichung der Forschungsergebnisse, geschweige denn eine gegenseitige Validierung möglich (Garz \& Kraimer, 1991).

Ungeachtet dieser eher an erkenntnistheoretischen und philosophischen Standpunkten orientierten Debatte über qualitative versus quantitative Forschung halten pragmatisch ausgerichtete Wissenschaftler daran fest, dass eine Integration der Forschungsrichtungen möglich und ertragreich ist. Der eine Forschungsansatz soll dabei weniger als Mittel der Validierung für den anderen Forschungsansatz dienen, sondern es wird vielmehr eine ,additive Verbindung“ (Fromm, 1990, S. 471) quantitativer und qualitativer Verfahren angestrebt. Durch eine Kombination der Forschungsansätze lässt sich nach Ansicht der Vertreter eines integrativen Ansatzes eine komplexere Sicht des Forschungsgegenstandes herstellen, nicht unbedingt jedoch eine objektivere (vgl. Rank, 1992).

\subsection{Methodische Standortbestimmung und Implikationen für die vorliegende Studie}

Richtungsweisend für die Methodenwahl in der vorliegenden Arbeit waren die Überlegungen von Pope und Mays (1995) und Baum (1995), dass die Anwendung eines bestimmten methodischen Ansatzes nicht von grundsätzlichen Erwägungen sondern von den Merkmalen des Forschungsgegenstandes und der Fragestellung abhängig gemacht werden sollte. Für die Ziele dieser Studie erschien ein Vorgehen, das quantitative und qualitative Forschungsansätze vereint, am geeignetesten.

Zwei Hauptanliegen verfolgt diese Studie: Zum einen sollte die Effektivität des Angehörigenkurses anhand verschiedener Zielvariablen überprüft werden, die eine interindividuelle Vergleichbarkeit der Daten zulassen. Da diese Zielvariablen außerdem 
im Rahmen eines Kontrollgruppen-Längsschnittdesigns mit mehreren Messzeitpunkten erhoben werden sollten, wurden aus forschungsökonomischen Gründen quantitative Erhebungs- und Auswertungsverfahren angewandt. Zum anderen sollte beleuchtet werden, welche intrapsychischen und sozialen Bedingungen für das konkrete Bewältigungsverhalten der pflegenden Angehörigen eine Rolle spielen. Besonderes Augenmerk wurde dabei auf die Interaktionen innerhalb der Pflegedyade, die dynamische Entwicklung sowie auf den Kontext der Pflegebeziehung gelegt. Dazu wurde ein qualitativer Ansatz gewählt: Als geeignetste Vorgehensweise, um die Situationsdeutungen, Handlungsmotive und Selbstinterpretationen von pflegenden Angehörigen differenziert $\mathrm{zu}$ erheben, erschien das Interview. Quantitative Daten reduzieren soziale oder familiäre Prozesse stets auf Zahlen, sodass sich komplexe Aspekte unter Umständen nicht adäquat abbilden lassen. Außerdem werden gerade in Familien dysfunktionale und konfliktbehaftete Verhaltensweisen nach außen hin eher verborgen. Die streng bewachten Grenzen zu überschreiten, scheint im Rahmen qualitativer Forschung am ehesten durch den Aufbau einer vertrauensvollen, kommunikativen Beziehung mit dem Befragten erreichbar zu sein (Rank, 1992). Interviews erlauben den Teilnehmern ihre Erfahrungen in ihrer eigenen Sprache mitzuteilen. So lässt sich am ehesten ein Zugang zu den Lebenswelten der Teilnehmer realisieren. 


\section{Methode - Quantitative Untersuchung}

\subsection{Allgemeines Studiendesign}

Im quantitativen Untersuchungsteil erfolgte die Evaluation des Kurses anhand eines Längsschnitt-Kontrollgruppendesigns mit drei Messzeitpunkten. Dabei wurden Teilnehmer am Kurs mit einer Kontrollgruppe verglichen, die das Interventionsangebot nicht in Anspruch nahm. Mitglieder der Kontrollgruppe konnten jedoch die sonstigen Angebote im Rahmen der teilstationären Rehabilitation ihres erkrankten Angehörigen nutzen, wie Beratungsgespräche bei den Sozialarbeitern, Therapeuten, Ärzten und Neuropsychologen. Der Erhebungszeitraum umfasste insgesamt 14 Monate. Die quantitative Untersuchung wurde anhand eines standardisierten Fragebogens durchgeführt, Mitglieder der Treatmentgruppe füllten zusätzlich einen Fragebogen zur Prozess-Evaluierung des Kurses aus.

\subsection{Stichprobe}

Angehörige aller Schlaganfallpatienten im Zeitraum von Mai 1996 bis Mai 1999 wurden schriftlich zum Angehörigenkurs eingeladen und telefonisch oder persönlich kontaktiert, um die Bereitschaft zur Teilnahme festzustellen. Angehörige, die sich zum Kurs anmeldeten, bildeten die Treatmentgruppe $(\mathrm{N}=42)$. Angehörige von Schlaganfallpatienten, die eine Teilnahme ablehnten, konstituierten die Kontrollgruppe $(\mathrm{N}=43)$. Das Design entspricht somit einem quasiexperimentellen Vorgehen. Aus praktischen und ethischen Überlegungen wurde auf eine Randomisierung der Stichprobe verzichtet. Durch dieses Vorgehen konnte außerdem eine hohe ökologische Validität gewährleistet werden. Als Gründe für die Nicht-Teilnahme wurden unter anderem genannt: das erkrankte Familienmitglied nicht allein lassen zu wollen, zu große berufliche Einbindung, Terminschwierigkeiten sowie ein bereits ausreichender Informationsgrad. Die hier untersuchte Treatmentgruppe basiert auf Daten aus 6 Angehörigenkursen. Ein Vergleich der deskriptiven Baseline-Daten der Treatmentgruppe und der Kontrollgruppe (siehe Tabelle 1) zeigte, dass sich beide Gruppen systematisch in Bezug auf eine wichtige Variable unterschieden. 
Tabelle 1: Charakteristika der Gesamtstichprobe zum Messzeitpunkt 1

\begin{tabular}{lcccccc}
\hline Variable & \multicolumn{2}{c}{ Treatmentgruppe } & \multicolumn{3}{c}{ Kontrollgruppe } \\
& \multicolumn{2}{c}{ N $=42$} & & & & \\
& M & SD & Range & M & SD & Range \\
& & & & & & \\
& $\mathbf{5 9 . 9}$ & $\mathbf{7 . 6}$ & $\mathbf{4 0 - 7 2}$ & $\mathbf{5 8 . 1}$ & $\mathbf{1 0 . 6}$ & $\mathbf{4 0 - 7 8}$ \\
Alter des Angehörigen & $\mathbf{6 4 . 8}$ & $\mathbf{9 . 5}$ & $\mathbf{4 5 - 8 6}$ & $\mathbf{6 2 . 9}$ & $\mathbf{1 0 . 3}$ & $\mathbf{4 2 - 9 5}$ \\
Alter des Patienten & $\mathbf{3 2 . 1}$ & $\mathbf{5 7 . 2}$ & $\mathbf{2 - 2 7 7}$ & $\mathbf{2 9 . 8}$ & $\mathbf{5 1 . 6}$ & $\mathbf{1 - 1 9 5}$ \\
Dauer der Pflege (in Monaten) & Md: 9.0 & & & Md: 5.0 & & \\
Funktionelle Einschränkung & & & & & & \\
des Patienten (Barthel-Index) & $\mathbf{4 9 . 1}$ & $\mathbf{2 7 . 7}$ & $\mathbf{0 - 1 0 0}$ & $\mathbf{6 5 . 8}$ & $\mathbf{2 9 . 3}$ & $\mathbf{0 - 1 0 0}$ \\
& Md: 55.0 & & & Md: 70.0 & & \\
\hline
\end{tabular}

Anmerkungen: Angabe von Mittelwert (M), Standardabweichung (SD), Median (Md). Barthel-Index:

Summenwert, Einschätzung durch Angehörige.

Das Ausmaß der Pflegebedürftigkeit, gemessen anhand des Barthel-Index, war in der Treatmentgruppe $($ Median $=55.0)$ signifikant höher als in der Kontrollgruppe $($ Median $=70.0)($ Mann-Whitney-Test, $U=574.0, p<0.01)$. Aus diesem Grund wurden Treatmentgruppe und Kontrollgruppe parallelisiert (Rossi \& Freeman, 1993, S. 297311). Die Variable „Ausmaß der Pflegebedürftigkeit” diente dabei als Hauptkriterium, „Dauer der Pflege” und „Alter der Pflegepersonen” als nachrangige Kriterien. Für jede Person in der Treatmentgruppe wurde versucht einen Probanden in der Kontrollgruppe zu finden, der in Bezug auf die drei genannten Variablen die größte Übereinstimmung zeigte. Das Verfahren entsprach somit einer ex post individuellen Parallelisierung. Die ursprüngliche Stichprobengrösse von $\mathrm{N}=42$ für die Treatmentgruppe und $\mathrm{N}=43$ für die Kontrollgruppe verkleinerte sich durch die Parallelisierung auf jeweils $\mathrm{N}=26$.

Die Treatmentgruppe setzte sich aus 22 Ehefrauen/Lebenspartnerinnen, 3 Ehemännern/ Lebenspartnern sowie einer pflegenden Tochter zusammen. Die Kontrollgruppe bestand aus 20 Ehefrauen/Lebenspartnerinnen, 4 Ehemännern/Lebenspartnern, einem Sohn und einer Tochter. In der Treatmentgruppe waren 6 Personen berufstätig, 20 bereits berentet oder hatten ihre Berufstätigkeit für die Pflege des Angehörigen aufgegeben. Die Kontrollgruppe umfasste 9 Berufstätige und 17 nicht Berufstätige. Aus Tabelle 2 sind die weiteren Charakteristika für die pflegenden Angehörigen der parallelisierten Stichproben zum Messzeitpunkt $1 \mathrm{zu}$ entnehmen. Tabelle 3 zeigt Merkmale der Pflegebedürftigen. 
Tabelle 2: Charakteristika der pflegenden Angehörigen (parallelisierte Teilstichproben) zum Messzeitpunkt 1

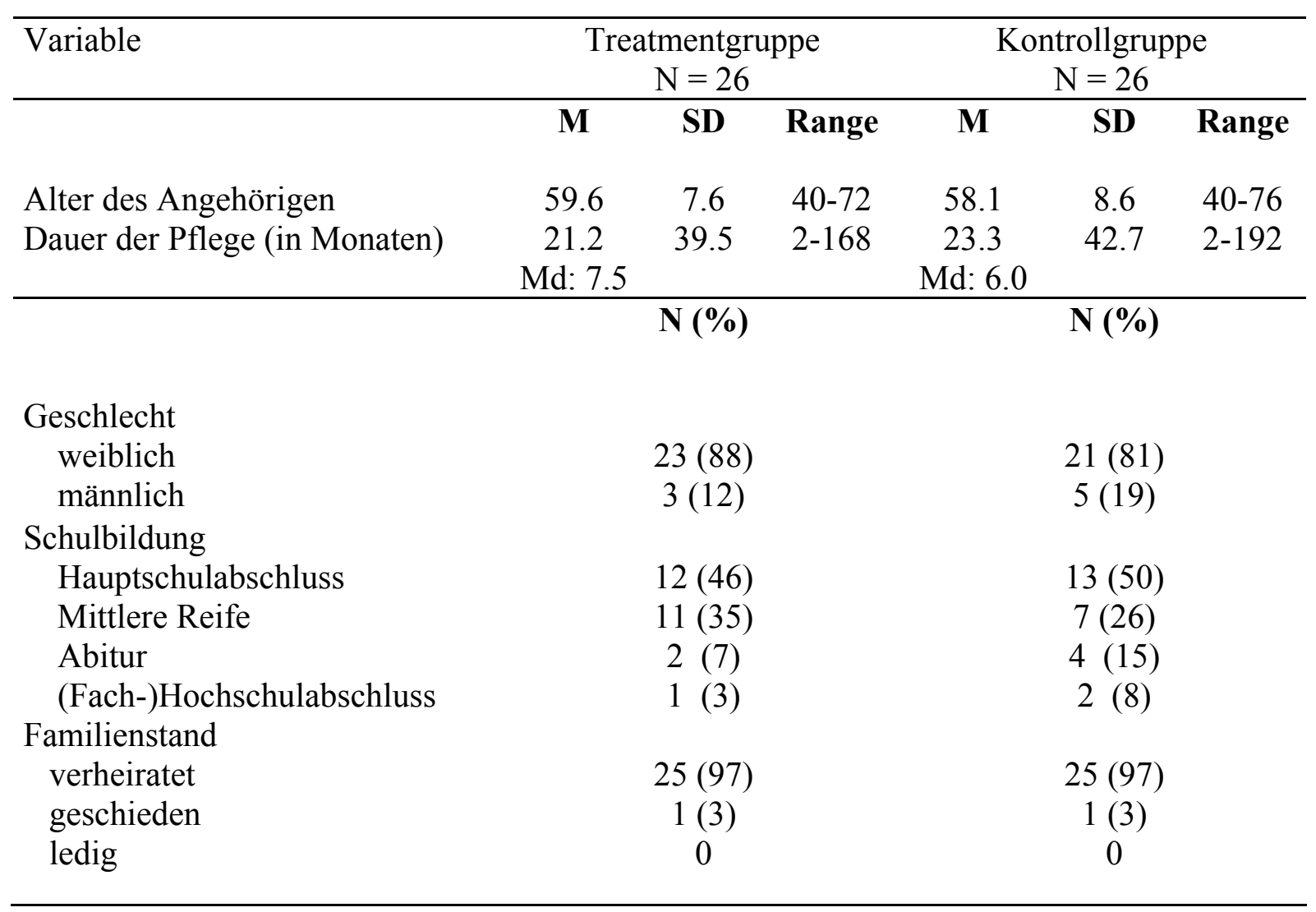

Anmerkungen: Angabe von Mittelwert (M), Standardabweichung (SD), Median (MD), absolute Häufigkeiten $(\mathrm{N})$. 
Tabelle 3: Charakteristika der Patienten (parallelisierte Teilstichproben) zum Messzeitpunkt 1

\begin{tabular}{|c|c|c|c|c|c|c|}
\hline \multirow[t]{2}{*}{ Variable } & \multicolumn{3}{|c|}{$\begin{array}{c}\text { Treatmentgruppe } \\
\mathrm{N}=26\end{array}$} & \multicolumn{3}{|c|}{$\begin{array}{l}\text { Kontrollgruppe } \\
\qquad \mathrm{N}=26\end{array}$} \\
\hline & $\mathbf{M}$ & SD & Range & $\mathbf{M}$ & SD & Range \\
\hline Alter des Patienten & 64.2 & 9.3 & $45-86$ & 62.1 & 10.5 & $42-95$ \\
\hline $\begin{array}{l}\text { Funktionelle Einschränkung } \\
\text { des Patienten (Barthel-Index) }\end{array}$ & $\begin{array}{c}51.2 \\
\text { Md: } 57.5\end{array}$ & 25.9 & $0-90$ & $\begin{array}{c}51.2 \\
\text { Md: } 55.0\end{array}$ & 26.5 & $0-100$ \\
\hline & & $\mathbf{N}(\%)$ & & & N (\%) & \\
\hline $\begin{array}{l}\text { Geschlecht } \\
\text { weiblich } \\
\text { männlich }\end{array}$ & & $\begin{array}{c}3(12) \\
23(88)\end{array}$ & & & $\begin{array}{l}4(16) \\
22(84)\end{array}$ & \\
\hline $\begin{array}{l}\text { Neuropsychologische } \\
\text { Beeinträchtigungen }\end{array}$ & & $18(69)$ & & & $16(61)$ & \\
\hline
\end{tabular}

Anmerkungen: Angabe von Mittelwert (M), Standardabweichung (SD), Median (MD), absolute Häufigkeiten (N). Barthel-Index: Summenwert, Einschätzung durch Angehörige. Neuropsychologische Defizite lagen vor, wenn in mindestens einem der 4 Bereiche Aufmerksamkeit, Gedächtnis, exekutive Funktionen und Abstraktionsvermögen zum Messzeitpunkt 1 Beeinträchtigungen nachweisbar waren. 


\subsection{Rücklaufquote}

Im Vergleich zu ähnlichen Studien konnte eine ausgesprochen hohe Rücklaufquote erzielt werden (siehe Tabelle 4), was durch verschiedene, in der Forschungsliteratur als wirksam beschriebene Maßnahmen, erreicht wurde (Friedrichs, 1990, S. 236-242). So wurden den Fragebögen z. B. jeweils eine handgeschriebene Karte mit individuell formuliertem Text und ein frankierter Rückumschlag beigefügt.

Tabelle 4: Rücklaufquote zu den Messzeitpunkten (MZP) 1, 2, 3

\begin{tabular}{lllll}
\hline & \multicolumn{2}{l}{ Treatmentgruppe } & \multicolumn{2}{l}{ Kontrollgruppe } \\
\hline & $\begin{array}{l}\text { ausgehändigt/ } \\
\text { verschickt }\end{array}$ & zurückgeschickt & $\begin{array}{l}\text { ausgehändigt/ } \\
\text { verschickt }\end{array}$ & zurückgeschickt \\
\hline MZP 1 & 54 & $50(92,6 \%)$ & 68 & $55(80,9 \%)$ \\
MZP 2 & 50 & $43(86,0 \%)$ & 55 & $48(87,3 \%)$ \\
\hline MZP 3 & 43 & $42(97,7 \%)$ & 48 & $43(89,6 \%)$ \\
\hline
\end{tabular}

\subsection{Messinstrumente}

Als Erhebungsinstrument diente im quantitativen Teil der Untersuchung ein standardisierter Fragebogen, der von den Studienteilnehmern vor Beginn des Kurses sowie 5 und 10 Monate nach dem ersten Messzeitpunkt ausgefüllt wurde (siehe Anhang A). Das Ausfüllen des Fragebogens nahm 30 bis 40 Minuten in Anspruch. Die Datenerhebung erfolgte anonym. Um eine Zuordnung der Fragebögen der verschiedenen Messzeitpunkte gewährleisten zu können, wurde von den Teilnehmern eine Codenummer auf der Titelseite des Fragebogens eingetragen.

Tabellen 5 und 6 geben einen Überblick über die verwendeten Messinstrumente. 
Tabelle 5: Verwendete Untersuchungsverfahren: Pflegende Angehörige

\begin{tabular}{|c|c|c|}
\hline Konstrukt & Teilaspekt & Messinstrument \\
\hline $\begin{array}{l}\text { Emotionale } \\
\text { Befindlichkeit }\end{array}$ & $\begin{array}{l}\text { - Depression } \\
\text { - Angst }\end{array}$ & $\begin{array}{l}\text { - Allgemeine Depressionsskala (ADS) } \\
\text { (Hautzinger \& Bailer, 1993) } \\
\text { - Self-Rating Anexiety Scale (SAS) } \\
\text { (Zung, 1971) }\end{array}$ \\
\hline $\begin{array}{l}\text { Gesundheitliche } \\
\text { Beeinträchtigung }\end{array}$ & $\begin{array}{l}\text { - Körperliche Beschwerden } \\
\text { - Medikamenteneinnahme } \\
\text { - Vorliegen einer chronischen } \\
\text { Erkrankung } \\
\text { - subjektive } \\
\text { Gesundheitseinschätzung }\end{array}$ & $\begin{array}{l}\text { - } \text { Beschwerdenliste (B-L) } \\
\text { (v. Zerssen, 1976) } \\
\text { - Mehrfachwahlskala } \\
\text { - } \text { offene Frage } \\
\text { - } \text { Ratingskala }\end{array}$ \\
\hline $\begin{array}{l}\text { Coping-Resourcen } \\
\text { interne Resourcen }\end{array}$ & $\begin{array}{l}\text { - subjektive Einschätzung des } \\
\text { Schlaganfallspezifischen Wissens }\end{array}$ & - Ratingskala (9 Items) \\
\hline externe Resourcen & $\begin{array}{ll}\text { - } & \text { soziale Unterstützung } \\
\text { - } & \text { finanzielle Ressourcen } \\
\text { - Hobbies, Aktivitäten }\end{array}$ & $\begin{array}{l}\text { - Ratingskala } \\
\text { - aus Patientenakte } \\
\text { - offene Fragen }\end{array}$ \\
\hline $\begin{array}{l}\text { Globale subjektive } \\
\text { Belastung }\end{array}$ & $\begin{array}{l}\text { - Einschätzung der subjektiven } \\
\text { Belastung }\end{array}$ & - Ratingskala (1 Item) \\
\hline $\begin{array}{l}\text { Soziodemo- } \\
\text { graphische Variablen }\end{array}$ & $\begin{array}{l}\text { - Alter, Geschlecht, Schulbildung, } \\
\text { Berufstätigkeit, sozioökonomischer } \\
\text { Status, Verwandtschaftsverhältnis, } \\
\text { Familienstand }\end{array}$ & $\begin{array}{l}\text { - Sozialanamnese aus dem } \\
\text { Geriatrischen Assessment }\end{array}$ \\
\hline
\end{tabular}


Tabelle 6: Verwendete Untersuchungsverfahren: Pflegerelevante Kennzeichen der Patienten

\begin{tabular}{|c|c|c|}
\hline Konstrukt & Teilaspekt & Messinstrument \\
\hline $\begin{array}{l}\text { Funktionaler Status } \\
\text { des Patienten }\end{array}$ & 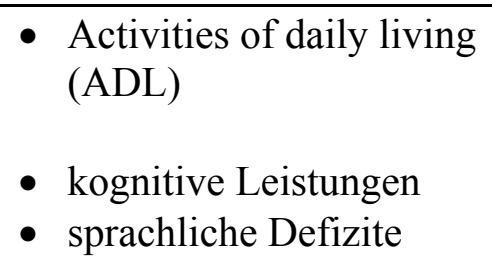 & 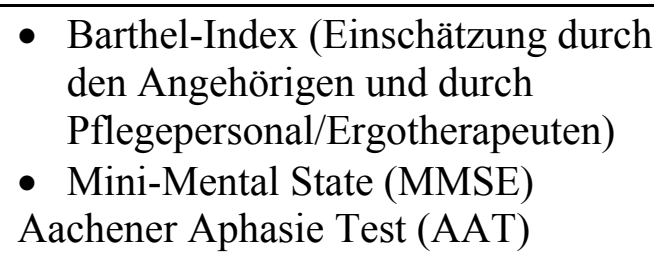 \\
\hline $\begin{array}{l}\text { Emotionale } \\
\text { Befindlichkeit des } \\
\text { Patienten }\end{array}$ & - Depression & - Geriatric Depression Scale (GDS) \\
\hline $\begin{array}{l}\text { Soziodemo- } \\
\text { graphische Variablen }\end{array}$ & $\begin{array}{l}\text { - Alter } \\
\text { - Geschlecht }\end{array}$ & - Angaben aus der Patientenakte \\
\hline
\end{tabular}

\section{Soziodemographische und pflegebezogene Daten}

Am Anfang des Fragebogens wurden demographische Daten erfragt (Alter, Geschlecht, Schulbildung, Berufstätigkeit etc.). Es folgten Angaben zu den Versorgungstätigkeiten und Hilfeleistungen, die der Angehörige ausführt. Des Weiteren wurde nach der Anzahl der Stunden, in denen konkrete Pflegetätigkeiten geleistet wurden, gefragt sowie nach der Anzahl der Stunden, die der Angehörige für den Pflegebedürftigen erreichbar bzw. anwesend sein muss.

\section{Gesundheitliche Beeinträchtigung}

\section{Körperliche Beschwerden}

Körperliche Beschwerden wurden anhand der Beschwerden-Liste (von Zerssen, 1976) erhoben. Dieses Messinstrument dient zur „quantitativen Abschätzung subjektiver Beeinträchtigung durch (überwiegend) körperliche und Allgemeinbeschwerden" (v. Zerssen, 1976, S. 6). Es umfasst insgesamt 24 Items, die sich inhaltlich in drei Bereiche unterteilen lassen: 
1. Allgemeinbeschwerden: z. B. Schwächegefühl, Müdigkeit, Schlafstörungen;

2. Lokalisierbare körpernahe Beschwerden: z. B. Sodbrennen, Gliederschmerzen;

3. Psychisch-körpernahe Beschwerden: z. B. innere Gespanntheit, Unruhe, Konzen-trationsmangel.

Anhand des Messinstrumentes kann eine Zustandsdiagnose, nicht aber eine nosologische Diagnose gestellt werden. Koloska, Rehm, \& Fichter (1989) zeigten in einer hypothesengeleiteten Analyse, dass der Beschwerdesymptomatik am ehesten eine Coping-relevante Dimension zugrunde liegt. Nach Aussage der Autoren kann somit die Höhe des Summenwertes der Beschwerden-Liste auch als Gradmesser für psychische Überforderung gewertet werden. Bei der Interpretation der Testergebnisse empfiehlt von Zerssen zwar die Trennung "normaler" von "abnormen" Testwerten, ein verbindlicher kritischer Wert wird jedoch nicht vorgegeben. In der vorliegenden Arbeit wurde als kritischer Grenzwert ein Rohwert von $>24$ festgelegt, dies entspricht einer Standardabweichung vom Mittelwert der Eichstichprobe.

Der Fragebogen weist gute Teststatistiken auf (interne Konsistenz: $r=0.90$, Paralleltest-Korrelation: $\mathrm{r}=0.85$ in der Eichstichprobe und $\mathrm{r}=0.88$ in einer psychiatrischen Vergleichsgruppe). Die kriterienbezogene Validität, die anhand eines Vergleichs einer klinischen Gruppe mit einer Kontrollgruppe erhoben wurde, beträgt laut Testmanual $r_{t c}=0.62$.

\section{Medikamenteneinnahme}

Als zusätzliches Maß für die Beeinträchtigung der Gesundheit wurde erhoben, welche Medikamente vorbeugend oder gegen bestimmte Beschwerden in den vergangenen vier Wochen eingenommen wurden. Als Antwortkategorien waren vorgegeben: Schmerzmittel, Herz-Kreislauf-Medikament, Beruhigungsmittel, Medikament gegen Infektionskrankheiten, Schlafmittel, Mittel zur Stärkung oder Aktivierung und Sonstiges.

\section{Vorliegen einer chronischen Erkrankung}

Um einen Hinweis darauf zu erhalten, inwieweit die Medikamenteneinnahme oder körperlichen Beschwerden durch das Bestehen einer chronischen Erkrankung 
beeinflusst sein könnte, folgte eine offene Frage nach dem Vorliegen einer derartigen Krankheit.

\section{Depression}

Die Variable Depression wurde anhand der Allgemeinen Depressions-Skala (ADS) (Hautzinger \& Bailer, 1993) operationalisiert. Die ADS, eine deutsche Version der Center for Epidemiologic Studies Depression Scale (CES-D) von Radloff (1977), eignet sich vor allem zur Erfassung depressiver Beschwerden im Vorfeld klinisch manifester depressiver Erkrankungen. Die Skala beinhaltet 20 (15 in der Kurzform) als Aussagen formulierte Items. Die Statements sind auf einer vierstufigen Skala von $0=$,,selten oder überhaupt nicht” bis $3=$,meistens, die ganze Zeit” einzuschätzen. Dabei werden folgende Dimensionen erhoben:

1. Gedrückte Stimmung;

2. Schuldgefühle;

3. Gefühl der Wertlosigkeit;

4. Gefühle von Hilf- und Hoffnungslosigkeit;

5. Antriebsmangel;

6. Appetitverlust;

7. Schlafstörungen.

Die Skala hat sich als reliables Selbstbeurteilungsinstrument erwiesen. In einer Bevölkerungsstichprobe mit 1298 Personen lag die innere Konsistenz bei $\alpha=0.89$ (Cronbach's Alpha). Die Testhalbierungs-Reliabilität betrug $r=0.81$. Der Mittelwert für den ADS-Summenwert lag in der Bevölkerungsstichprobe bei $\mathrm{M}=14.3$, die Standardabweichung war SD = 9.7 (Hautzinger \& Bailer, 1991, S. 11-14).

Die Autoren schlagen für die ADS-Langform, die in der vorliegenden Studie verwendet wurde, einen kritischen Grenzwert von $>23$ vor; dies entspricht einer Standardabweichung vom Mittelwert der Eichstichprobe. 


\section{Angst}

Als Messinstrument für das Vorliegen von Angst-Symptomen diente die Self-Rating Anxiety Scale (SAS) von Zung (1971). Die SAS enthält 20 Kriterien für Angst, von denen 5 affektiv und 15 somatisch sind. Die Items sind von den Studienteilnehmern nach ihrer Auftretenshäufigkeit in den letzten 7 Tagen einzuschätzen. Die Antwortalternativen umfassen ,nie oder selten”, „manchmal”, „öfters” und „meistens oder immer”. Beispielitems sind: „Ich fühle mich nervöser und ängstlicher als sonst”, „Ich schlafe leicht ein und finde erholsamen Schlaf” oder „Ich kann spüren, wie mein Herz ganz schnell pocht." Der Gesamtrohwert errechnet sich aus der Summe aller Items. Das Manual gibt einen Summenwert von $>35$ als kritischen Grenzwert an.

Die Skala weist eine hohe kriterienbezogene Validität auf. Der mittlere SASGesamtwert lag bei Patienten mit der Diagnose einer Angststörung signifikant höher (p $\leq 0.05)$ als bei Patienten mit anderen psychiatrischen Diagnosen. Die Korrelation zwischen der Selbstbeurteilung von Probanden und einer Fremdbeurteilung (Einschätzung durch einen Psychiater) anhand der Skala lag bei $r=0.74$. Auch die Zuverlässigkeit des Messinstrumentes kann als gut angesehen werden, die Testhalbierungs-Reliabilität betrug $r=0.71$ (Zung, 1971).

\section{Coping-Ressourcen}

Positive Ressourcen stellen nach Roskies und Lazarus (1980) wichtige Voraussetzungen für effiziente Copingprozesse im Sinne der Ausschaltung der Stressoren oder der Adaptation des Individuums an die Stressoren dar. Dabei lassen sich externe Ressourcen (z. B. soziale Unterstützung) von internen Ressourcen (z. B. Wissen, Problemlösefähigkeiten) unterscheiden.

\section{Externe Ressourcen}

\section{Soziale Unterstïtzung}

Es wurde nach verschiedenen Bereichen der sozialen Unterstützung durch Freunde, Bekannte oder Familienangehörige gefragt. Diese waren im einzelnen: Erledigen von Einkäufen, hauswirtschaftliche Tätigkeiten (z. B. Putzen, Kochen), Fahrdienste (z. B. zum Arzt), Besuche, Zuspruch und Trost. Antwortvorgaben waren „nein”, 
„gelegentlich” und „öfter”. Zusätzlich wurde erfasst, ob sich die pflegende Angehörige auf diese Unterstützung verlassen könne und ob sie sich mehr Unterstützung in den genannten Bereichen wünschte.

\section{Nutzung (psycho-)sozialer Entlastungsangebote}

Außerdem wurde erhoben, ob und welche (psycho-)sozialen Entlastungsangebote von den Angehörigen genutzt wurden. Dazu zählten: Pflegedienst, Mobilitätsdienst, private Haushaltshilfe, fahrbarer Mittagstisch, Zivildienstleistender, Tagespflegestätte, Kurzzeitpflege und der Besuch einer Gesprächsgruppe.

\section{Positiver Ausgleich}

Für die Erfassung eines positiven Ausgleichs zur Pflege wurden die Probanden gebeten, alle Hobbies und Interessen aufzuzählen, denen sie regelmäßig nachgingen. Des Weiteren wurde danach gefragt, wie sich die Interessen in letzter Zeit entwickelten und ob die Angehörigen damit zufrieden seien. Dies war anhand einer 4- bzw. 3-stufigen Skala einzuschätzen.

\section{Finanzielle Situation}

Als weitere externe Ressource wurde zusätzlich zum sozioökonomischen Status aus der Patientenakte die subjektive Einschätzung der finanziellen Situation durch den Angehörigen erhoben. Wortlaut der Frage war: „Machen Sie sich Sorgen über Ihre gemeinsame finanzielle Zukunft oder über die Ihres Angehörigen?" Die Antwortvorgabe bestand in einer 4-stufigen Skala, die von ,selten oder nie” bis „meistens oder immer” reichte.

\section{Interne Ressourcen}

\section{Schlaganfallspezifisches Wissen}

Schlaganfallspezifisches Wissen wurde als eine wichtige interne Ressource für ein effektives Coping angesehen und wurde als subjektive Einschätzung des Informiertheitsgrades operationalisiert. Es wurden 9 Bereiche aufgeführt, die für den Umgang mit einer Schlaganfallerkrankung relevant sind. Diese umfassten: 
1. Medizinische Ursachen der Erkrankung

2. Medikamentöse Behandlung

3. Hilfsmittel

4. Sprachstörungen

5. Hirnleistungsstörungen

6. Entlastungsmöglichkeiten durch soziale Dienste

7. Pflegeversicherung

8. Physiotherapeutische Behandlungsmethoden

9. Ergotherapeutische Behandlungsmethoden

Die Studienteilnehmer wurden gebeten anzugeben, wie gut sie sich in diesen Bereichen informiert fühlten. Als Antwortvorgabe diente eine 5-stufige Skala, die von ,sehr gut informiert” bis ,überhaupt nicht informiert” reichte.

\section{Subjektive Belastung durch die Pflege}

Das globale subjektive Belastungserleben wurde anhand der Frage „Wie stark fühlen Sie sich durch die Erkrankung Ihres Angehörigen belastet?" erhoben. Die 5-stufige Antwortskala hatte die Endpunkte ,sehr stark belastet” und „nicht belastet”.

\section{Pflegerelevante Kennzeichen der Patienten}

Um einzuschätzen zu können, inwieweit bestimmte Kennzeichen des Patienten Auswirkungen auf die Pflegesituation hatte, wurden zusätzlich diverse pflegerelevante Aspekte ermittelt. Dies geschah bei Aufnahme des Patienten im Rahmen des Geriatrischen Assessment (Arbeitsgruppe Geriatrisches Assessment - AGAST, 1995).

\section{Ausmaß der Pflegebedürftigkeit}

Der funktionelle Status des Patienten (ADL-Status) wurde anhand des Barthel-Index (Mahoney \& Barthel, 1965) erhoben. Dieser misst die Fähigkeit zur selbständigen Lebensführung in den Bereichen „Essen”, „Bett/(Roll-)Stuhltransfer”, „Waschen”, „Toilettenbenutzung”, „Baden”, „Gehen auf Flurebene/Rollstuhlfahren”, „Treppensteigen”, „An- und Auskleiden”, „Stuhlkontrolle” und „Urinkontrolle”. Zu jedem Bereich wird das Ausmaß der Selbständigkeit anhand eines Punktwertes von 0, 5, 
10 oder 15 eingeschätzt, wobei jede Stufe eindeutig definiert ist. Der Gesamtpunktwert kann von 0 bis 100 variieren, höhere Werte deuten auf größere Selbständigkeit hin. In einer Studie von Granger, Albrecht und Hamilton (1979) konnte eine Retest-Reliabilität von $r=0.89$ ermittelt werden, die Inter-Rater-Übereinstimmung lag bei $r=0.95$.

Die Einschätzung des Barthel-Index erfolgte in der vorliegenden Arbeit durch die pflegenden Angehörigen.

\section{Kognitiver Status}

Zur Erfassung des kognitiven Abbaus der Patienten wurde die Mini-Mental-StateExamination (MMSE) (Folstein, Folstein \& McMugh, 1975) eingesetzt, die im Rahmen des Geriatrischen Assessment als Screening Instrument von den Neuropsychologen verwendet wird. Die MMSE ist das weltweit am häufigsten angewandte Screeningverfahren für dementielle Erkrankungen. Überprüft werden die Bereiche Orientiertheit, Gedächtnis, Aufmerksamkeit, Benennen, Lesen, Schreiben sowie visuokonstruktive Fähigkeiten. Ein Wert von 24 oder weniger weist mit hoher Wahrscheinlichkeit auf eine kognitive Einschränkung hin.

\section{Sprachliche Funktionen}

Die sprachlichen Funktionen wurden im Rahmen des logopädischen Aufnahmebefundes überprüft. Dabei wurde unter anderem der Token Test, ein Auslesetest zur Differenzierung von Aphasikern und Nichtaphasikern, durchgeführt (Orgass, 1982).

\section{Depression}

Die Einschätzung einer depressiven Symptomatik der Patienten erfolgte durch die Neuropsychologen in der Tagesklinik im Rahmen des Geriatrischen Assessment, das bei Aufnahme durchgeführt wird. Dabei wird die Kurzversion der Geriatric Depression Scale (GDS von Yesavage, Brink, Rose et al., 1983) verwendet, die von der Arbeitsgruppe „Geriatrisches Assessment” (AGAST) als Screeningverfahren empfohlen wird (Hofmann, Nikolaus, Pientka \& Stuck, 1995). Mittlerweile wird die GDS standardmäßig in vielen geriatrischen Einrichtungen sowie im ambulanten Bereich eingesetzt. Die Skala besteht aus 15 Items, die vorrangig die psychologischen Aspekte der Depression erfassen. Ein Rohwert von $>6$ spricht für das Vorliegen einer 
depressiven Symptomatik. Die Skala ist in hohem Maße sensitiv und reliabel (Rapp, Parisi, Walsh \& Wallace, 1988) und korreliert mit dem Beck-Depressions-Inventar (Beck, Ward, Mendelson, Mock \& Erbaugh, 1961) mit $\mathrm{r}=0.74$ und mit der Allgemeinen Depressionsskala mit $r=0.70$ (Gauggel, Schmidt \& Didié, 1994).

\section{Subjektiv erlebter Nutzen des Kurses}

Ein weiterer Fragebogen wurde nur von den Probanden in der Treatmentgruppe zum Ende des Kurses ausgefüllt (siehe Anhang A). Dieser enthielt Fragen zum subjektiv erlebten Nutzen des Kurses und zur Bewertung der einzelnen Kursabende. Im ersten Teil des Fragebogens wurden die Teilnehmer gebeten, 15 Statements zum Kurs anhand einer 4-stufigen Skala (von „stimmt nicht” bis „stimmt genau”) zu bewerten.

Beispiel-Items sind:

„Ich konnte mich mit anderen austauschen.”

„Die Broschüren und Übersichtspapiere waren insgesamt gut verständlich.”

„Ich würde den Kurs anderen betroffenen Angehörigen weiterempfehlen.”

Im zweiten Teil des Fragebogens wurden die Angehörigen gebeten, jede Kurseinheit zu bewerten und anzugeben, welche Inhalte zusätzlich erwünscht gewesen wären. Die letzte Seite des Fragebogens gab den Teilnehmern die Möglichkeit, Verbesserungsvorschläge oder Anregungen für weitere Kurse, negative und positive Kritik zu äußern.

\subsection{Statistische Auswertung}

Die Auswertung der Daten erfolgte mit dem Statistikprogramm SPSS für WINDOWS (Version 10.0). Da die Daten der Outcome-Variablen in den meisten Fällen nicht normalverteilt waren, wurden durchgängig nonparametrische Verfahren zur Überprüfung von Unterschieden zwischen den Gruppen und innerhalb der Gruppen über die verschiedenen Messzeitpunkte hinweg angewandt. Die statistischen Prüfungen erfolgten stets zweiseitig. 


\section{Ergebnisse - Quantitative Untersuchung}

\subsection{Beeinträchtigungen des psychischen und körperlichen Wohlbefindens der Angehörigen}

In der Depressionsskala (ADS) weisen die untersuchten Angehörigen zu allen Messzeitpunkten im Mittel erhöhte Werte auf (siehe Tabelle 7). Insgesamt liegen die Mittelwerte in beiden Gruppen zwar noch unterhalb des pathologischen Grenzwertes (22 Punkte), sie sind aber deutlich höher als in der Normalbevölkerung ( $\mathrm{M}=14.3$, Hautzinger \& Bailer, 1993). Die Werte in der Kontrollgruppe liegen zu allen Messzeitpunkten niedriger als in der Treatmentgruppe, dieser Unterschied ist jedoch nicht statistisch signifikant (Mann-Whitney-Test). Im zeitlichen Verlauf zeigen sich weder in der Treatment- noch in der Kontrollgruppe signifikante Unterschiede (Friedman-Test).

Tabelle 7: Psychisches und körperliches Befinden der pflegenden Angehörigen für Messzeitpunkte 1, 2 und 3, $\mathrm{N}=26$ pro Gruppe

\begin{tabular}{lcccccc}
\hline & \multicolumn{5}{c}{ Messzeitpunkte } \\
& \multicolumn{1}{c}{$\mathbf{1}$} & \multicolumn{2}{c}{$\mathbf{2}$} & \multicolumn{3}{c}{} \\
& & & (+ 5 Monate) & (+ 10 Monate) \\
\hline & M & SD & M & SD & M & SD \\
\hline Depression (ADS) & & & & & & \\
Treatmentgruppe & $\mathbf{2 1 . 8}$ & 10.9 & $\mathbf{2 0 . 0}$ & 9.6 & $\mathbf{2 1 . 2}$ & 11.8 \\
Kontrollgruppe & $\mathbf{1 8 . 9}$ & 9.3 & $\mathbf{1 5 . 3}$ & 8.9 & $\mathbf{1 6 . 4}$ & 9.8 \\
Angst (SAS) & & & & & & \\
Treatmentgruppe & $\mathbf{3 9 . 1}$ & 10.0 & $\mathbf{3 8 . 2}$ & 9.0 & $\mathbf{3 8 . 2}$ & 10.9 \\
Kontrollgruppe & $\mathbf{3 5 . 0}$ & 9.0 & $\mathbf{3 4 . 4}$ & 9.1 & $\mathbf{3 6 . 2}$ & 8.9 \\
körperl. Beschwerden (B-L) & & & & & & \\
Treatmentgruppe & $\mathbf{2 9 . 2}$ & 13.5 & $\mathbf{2 9 . 2}$ & 11.7 & $\mathbf{3 0 . 0}$ & 14.5 \\
Kontrollgruppe & $\mathbf{2 5 . 9}$ & 12.5 & $\mathbf{2 7 . 5}$ & 12.9 & $\mathbf{2 7 . 5}$ & 13.4 \\
\hline
\end{tabular}

Anmerkung: ADS = Allgemeine Depressionsskala, Werte oberhalb 22 Punkte gelten als auffällig; SAS = Self-Rating Anxiety Scale, Cutoff-Wert: 35; B-L = Beschwerden-Liste, Cutoff-Wert: 24. 
In der Angstskala (SAS) liegen sowohl in der Treatmentgruppe als auch in der Kontrollgruppe die Werte zu allen Messzeitpunkten deutlich höher als in der Normalbevölkerung. In der Referenzgruppe der 20 bis 64-jährigen liegt der Mittelwert bei 27.2, in der Gruppe der über 64-jährigen bei 32.0 (Zung, 1971). Während in der Treatmentgruppe die Mittelwerte $\mathrm{zu}$ allen Messzeitpunkten oberhalb des kritischen Wertes (35 Punkte) liegen, überschreitet der Mittelwert in der Kontrollgruppe erst zum Messzeitpunkt 3 den Cutoff (siehe Tabelle 7). Allerdings erreicht dieser Anstieg keine statistische Signifikanz (Friedman-Test). Auch in der Treatmentgruppe konnte im zeitlichen Verlauf keine signifikante Veränderung beobachtet werden (Friedman-Test).

Die Gruppenmittelwerte für die körperliche Befindlichkeit, gemessen anhand der Beschwerden-Liste (B-L) (von Zerssen, 1976), liegen jeweils deutlich über dem kritischen Wert von 24 Punkten (siehe Tabelle 6), in beiden Gruppen besteht also ein erhöhtes Ausmaß an körperlichen Beschwerden. Die Mittelwerte in der Treatmentgruppe sind vergleichbar mit dem Testmittelwert einer psychiatrischen Vergleichsgruppe $(M=30.0$, von Zerssen, 1976). Eine statistisch signifikante Veränderung des Ausmaßes der körperlichen Beschwerden im zeitlichen Verlauf ist weder in der Treatment- noch in der Kontrollgruppe festzustellen (Friedman-Test).

Die globale subjektive Belastung wird in beiden Gruppen im Mittel als recht hoch angegeben, der Ausgangswert zum Messzeitpunkt 1 liegt jeweils über dem Skalenmittelpunkt von 3. Für die Treatmentgruppe ist im zeitlichen Verlauf eine signifikante Abnahme der subjektiven Belastung festzustellen (Friedman-Test, $\chi^{2}=9.7$, $\mathrm{p}=0.008$ ). In der Kontrollgruppe verändert sich die globale subjektive Belastung im zeitlichen Verlauf dagegen nicht signifikant. Liegt der Mittelwert für die Treatmentgruppe $(\mathrm{M}=4.1)$ zum Messzeitpunkt 1 noch deutlich höher als in der Kontrollgruppe $(\mathrm{M}=3.4$, Mann-Whitney-Test, $\mathrm{U}=213.5, \mathrm{p}=0.01)$, ist dieser Unterschied zum Messzeitpunkt 3 nicht mehr zu ermitteln (vgl. Abbildung 5). 


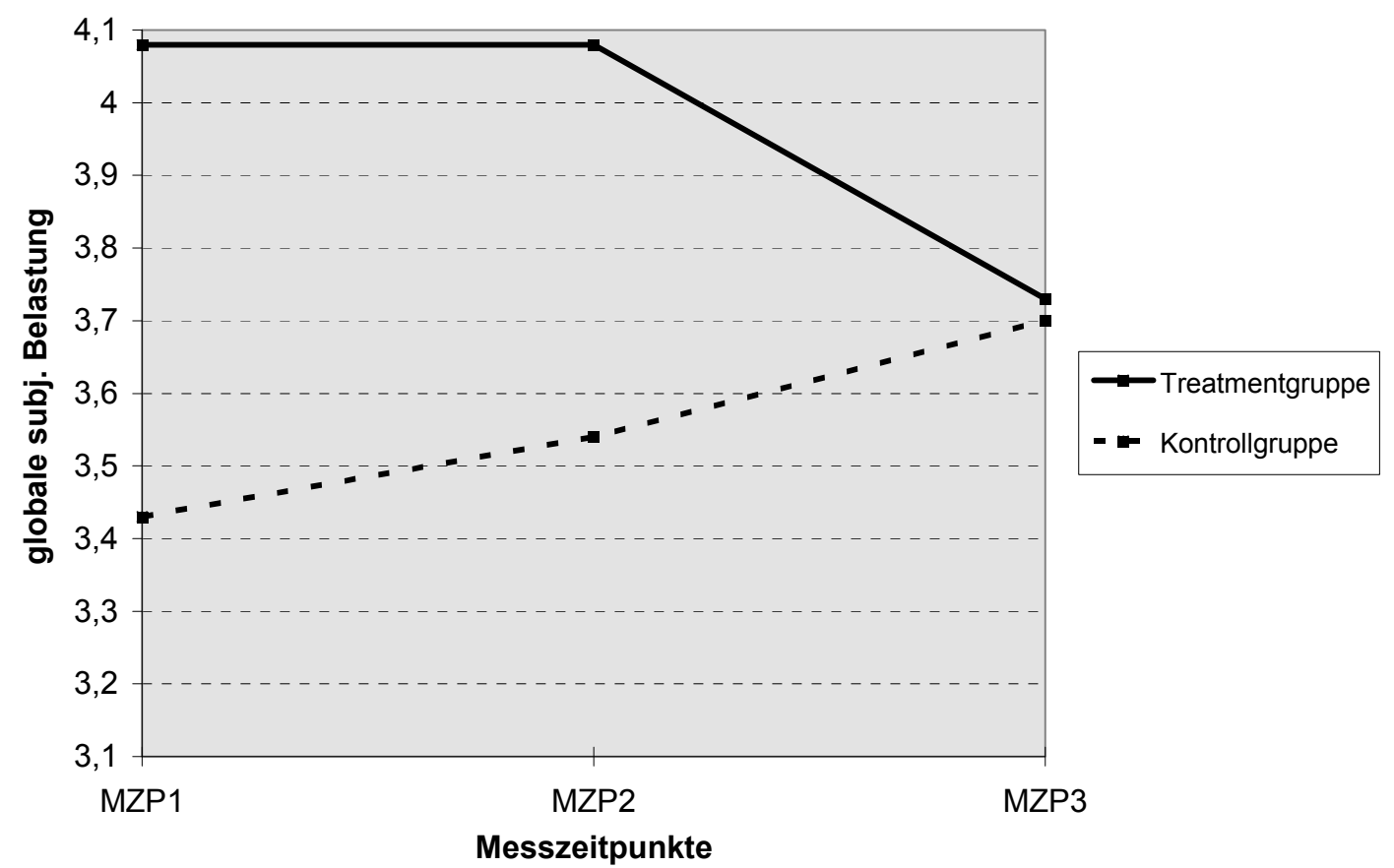

Abbildung 5: Globale subjektive Belastung zu den Messzeitpunkten 1, 2, 3 für parallelisierte Stichproben (pro Gruppe $\mathrm{N}=26$ )

Anmerkung: Die globale subjektive Belastung wurde anhand einer 5-stufigen Skala gemessen mit den Endpunkten $1=$ nicht belastet und $5=$ sehr stark belastet.

\subsection{Veränderung des Informiertheitsgrades in Bezug auf Schlaganfall- spezifisches Wissen}

Zum Messzeitpunkt 1 ist für die Treatmentgruppe im Mittel $(\mathrm{M}=2.85)$ ein deutlich geringerer Informiertheitsgrad $\mathrm{zu}$ beobachten als in der Kontrollgruppe $(\mathrm{M}=3.52$, Mann-Whitney-Test, $U=181, \mathrm{p}=0.01)$. Im zeitlichen Verlauf nimmt das Schlaganfallspezifische Wissen jedoch in der Treatmentgruppe signifikant zu (Friedman-Test, $\mathrm{p}<0.000$ ), während es in der Kontrollgruppe nahezu konstant bleibt (Tabelle 8). 
Tabelle 8: Ausmaß des Schlaganfallspezifischen Wissens der pflegenden Angehörigen für Messzeitpunkte 1, 2 und 3, $N=26$ pro Gruppe

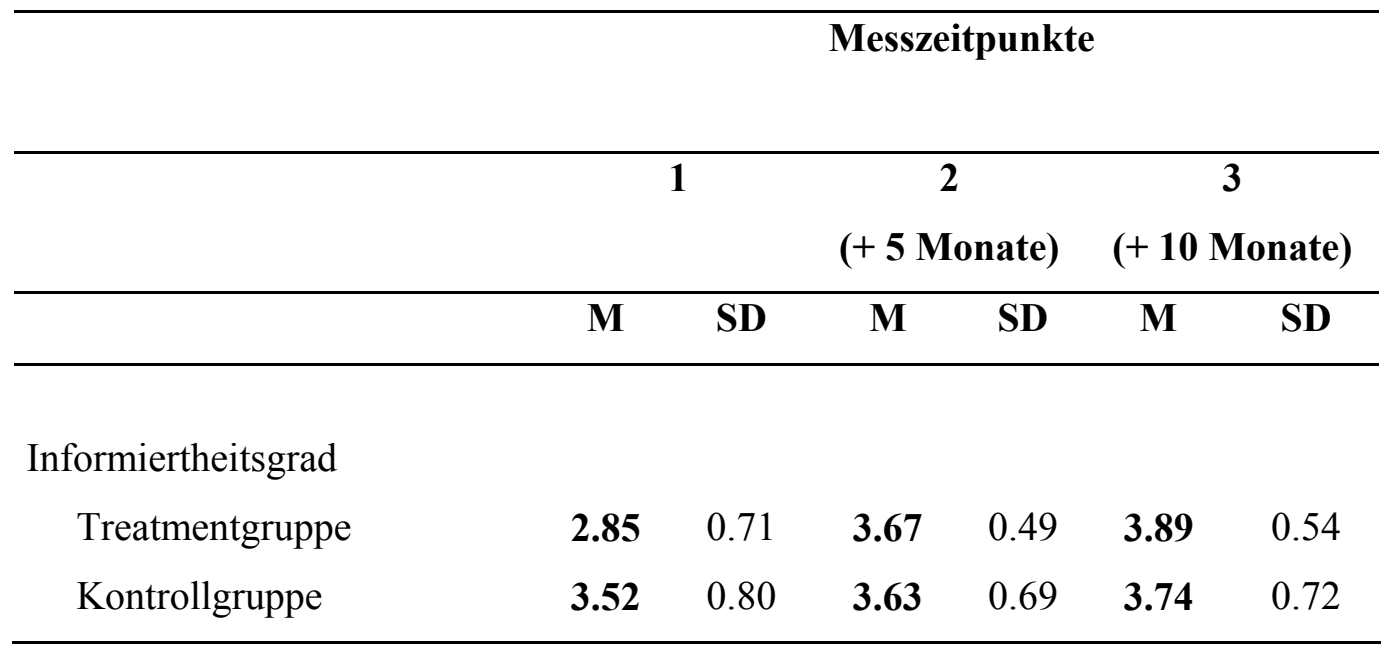

Anmerkungen: Der Informiertheitsgrad in Bezug auf Schlaganfall-spezifisches Wissen wurde anhand von 9 Themenbereichen ermittelt. Zur Einschätzung diente jeweils eine 5-stufige Skala mit den Endpunkten $1=$ überhaupt nicht informiert und $5=$ sehr gut informiert. Für die Datenauswertung wurde der Mittelwert über alle neun Aspekte hinweg gebildet.

\subsection{Nutzung (psycho-)sozialer Entlastungsangebote}

Nehmen zum ersten Messzeitpunkt nur 8 Angehörige in der Treatmentgruppe Entlastungsangebote in Anspruch, erhöht sich die Zahl nach Teilnahme am Kurs auf 24 bzw. 22 (Abbildung 6). Diese Zunahme in der Nutzung (psycho-)sozialer Entlastungsangebote im zeitlichen Verlauf erweist sich als signifikant (Cochran-Test, $\mathrm{p}<0.000$ ). In der Kontrollgruppe ist der Ausgangswert zum Messzeitpunkt 1 noch vergleichbar mit der Treatmentgruppe. Nur 6 Angehörige nehmen Entlastungsangebote in Anspruch. Im zeitlichen Verlauf ergibt sich im Gegensatz zur Treatmentgruppe jedoch keine signifikante Steigerung (vgl. Abbildung 6). 


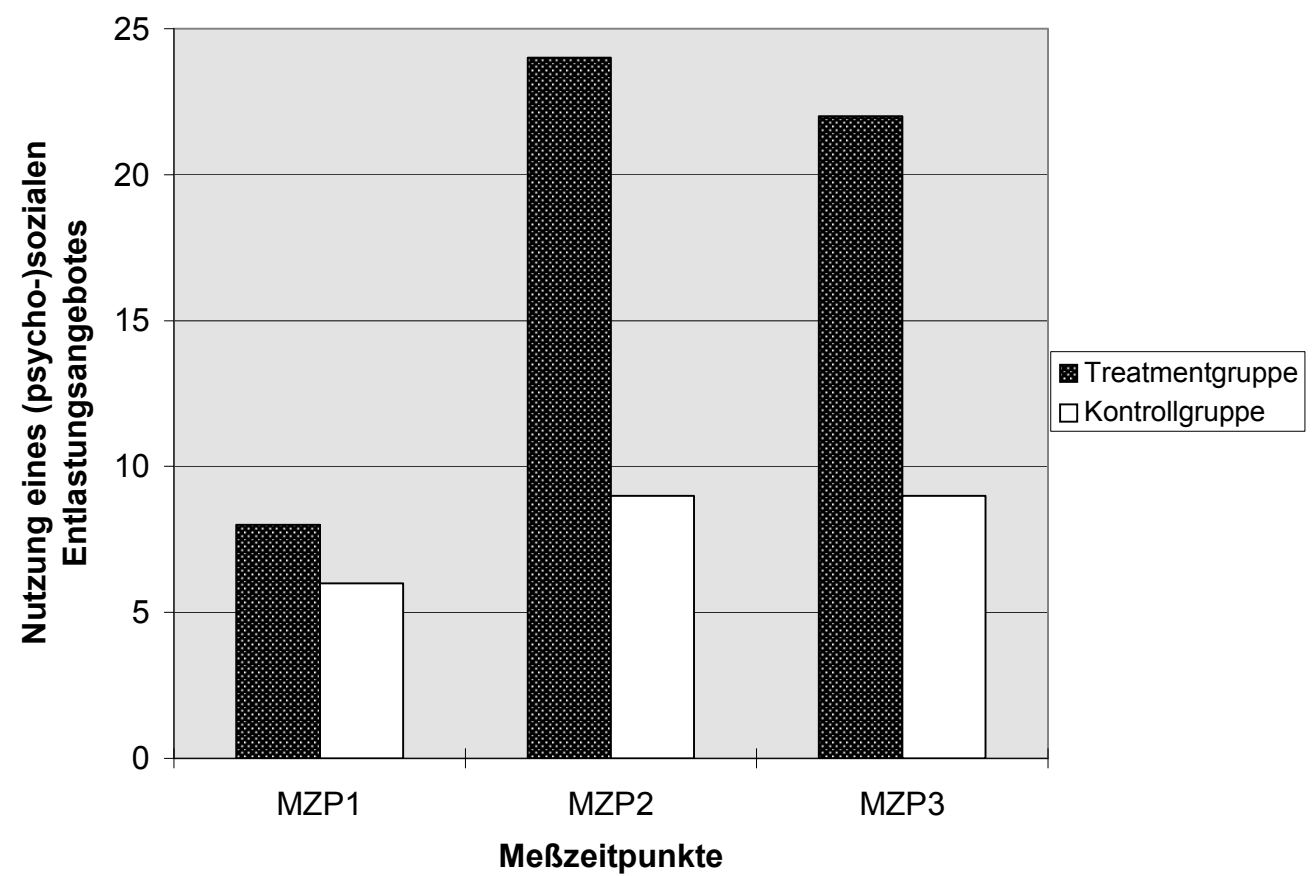

Abbildung 6: Nutzung psychosozialer Entlastungsangebote zu den Messzeitpunkten 1, 2 und 3 für parallelisierte Stichproben $(\mathrm{N}=26$ pro Gruppe)

Anmerkung: Angabe der Anzahl von Angehörigen, die mindestens ein (psycho-)soziales Entlastungsangebot nutzen.

\subsection{Subjektiv erlebter Nutzen des Kurses}

Zur subjektiven Bewertung des Kurses wurde ein weiterer Fragebogen nach Beendigung des Kurses eingesetzt (siehe Anhang A). Im ersten Teil des Fragebogens wurden die Teilnehmer gebeten, 14 Feststellungen zum Kurs anhand einer 4-stufigen Skala (von "stimmt nicht" bis "stimmt genau") zu bewerten. So sollten sie zum Beispiel angeben, ob sie sich mit anderen austauschen konnten, ob sie die Broschüren und Übersichtspapiere gut verständlich fanden und ob sie den Kurs anderen betroffenen Angehörigen weiterempfehlen würden. Die Auswertung ergab eine sehr positive Bewertung des Kurses, wie die Abbildung 7 zeigt. 


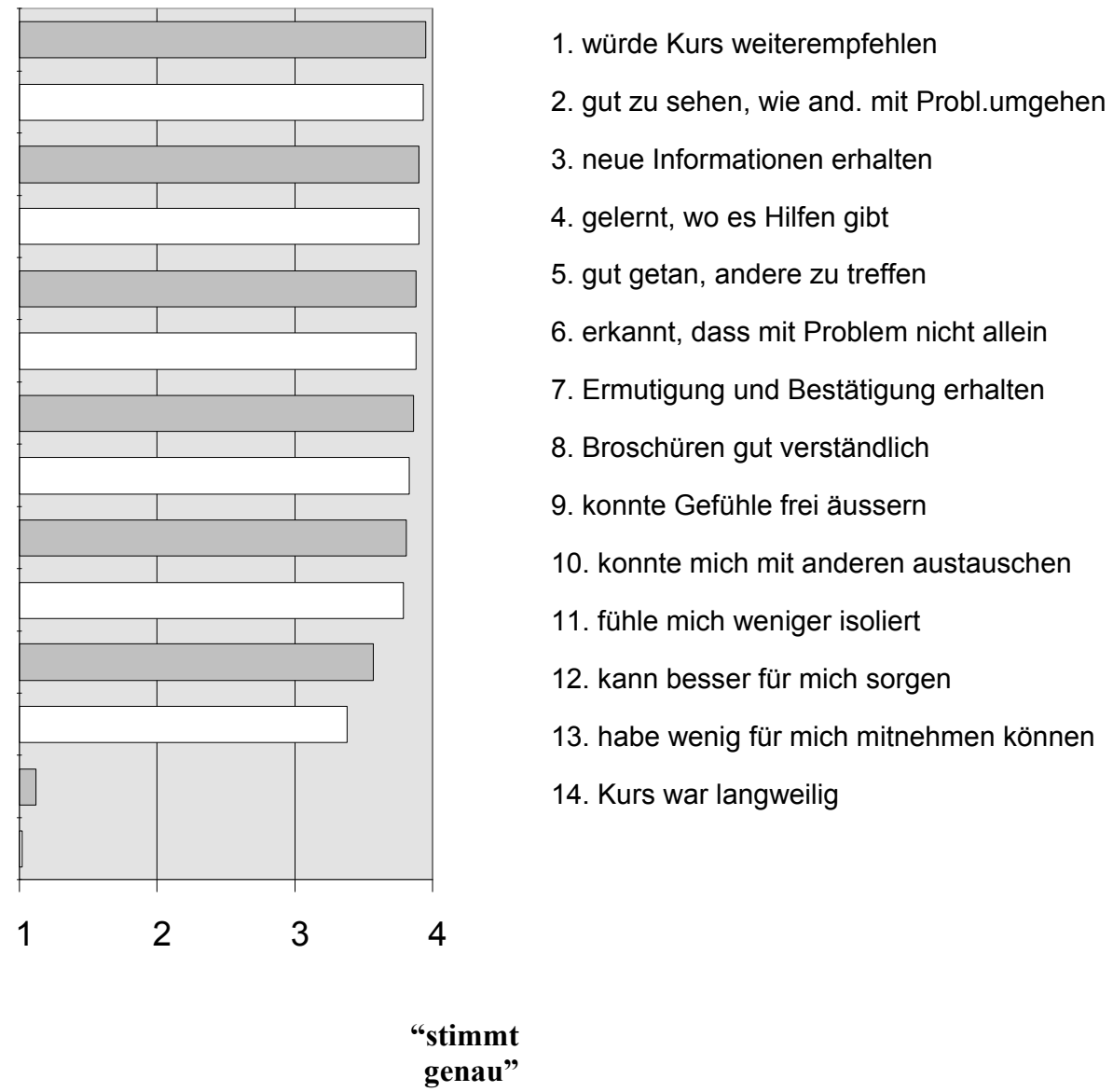

\footnotetext{
Abbildung 7: Subjektiv erlebter Nutzen des Angehörigenkurses für $\mathrm{N}=42$

Anmerkung: Abgebildet sind die jeweiligen Mittelwerte. Die Aussagen sollten jeweils anhand einer 4-stufigen Skala mit den Endpunkten $1=$ stimmt nicht und $5=$ stimmt genau bewertet werden.
}

Eine weitere offene Frage zielte darauf ab, welche Aspekte die Angehörigen aufgrund des Kurses in der Pflegesituation verändert hätten. Dazu machten 32 von 42 Teilnehmern des Kurses schriftliche Angaben. Die genannten Aspekte sind der folgenden Tabelle zu entnehmen. 
Tabelle 9: Von Angehörigen genannte Veränderungen in der Pflegesituation aufgrund der Teilnahme am Kurs

Aspekte der Veränderungen

$\mathrm{N}$

Schaffung größerer Freiräume für sich selbst, um eigenen Interessen 7 nachzugehen

stärkere Förderung der Selbständigkeit des Pflegebedürftigen

Befolgung der Hinweise bezüglich Lagerung nach dem Bobath-Konzept

stärkere Einbindung anderer Familienmitglieder in die Pflege

stärkere Einbeziehung des Pflegebedürftigen in den Alltag

Inanspruchnahme einer ausführlichen individuellen Beratung zu sozialen

Unterstützungsmöglichkeiten

Bessere Strukturierung des Tagesablaufs

Akzeptieren der Wesensveränderungen des Pflegebedürftigen als Folge der Krankheit

Beschaffung sinnvoller Hilfsmittel

Anmerkung: Zum Teil wurden mehrere Aspekte von einem Teilnehmer aufgeführt, sodass die Gesamtsumme der Nennungen $\mathrm{N}=32$ übersteigt.

Des Weiteren wurden Angehörige gebeten, Verbesserungsvorschläge und Anregungen für weitere Kurse $\mathrm{zu}$ nennen. Vier Angehörige wünschten sich Vertiefungen $\mathrm{zu}$ verschiedenen Themen (z. B. Ernährung, neuropsychologische Störungen, finanzielle und sozialrechtliche Fragen). Drei Angehörige forderten mehr Zeit für die praktischen Anleitungen, insbesondere bei der Demonstration von Transfer und Lagerung nach Bobath. Eine Angehörige schlug eine Verstärkung der Öffentlichkeitsarbeit vor, damit auch Angehörige von Patienten, die nicht in der Tagesklinik behandelt wurden, das Kursangebot wahrnehmen könnten. Von acht Angehörigen wurde die Fortführung der Gruppe als Gesprächsgruppe angeregt. Eine Angehörige wünschte sich einen gemeinsamen Kursabend mit dem erkrankten 
Angehörigen. Die Möglichkeit Dinge zu benennen, die ihnen nicht gefallen hatten, nutzen nur zwei Personen. Beide bemängelten zu große Redeanteile einzelner Kursteilnehmer.

Den Abschluss des Fragebogens bildete eine offene Frage nach guten und hilfreichen Aspekten des Kurses. Dazu machten 19 (von 42) Teilnehmern schriftliche Angaben. Die folgende Tabelle zeigt eine Übersicht.

Tabelle 10: Von Angehörigen genannte positive und hilfreiche Aspekte des Kurses

\begin{tabular}{lc}
\hline Positive und hilfreiche Aspekte des Kurses & $\mathrm{N}$ \\
\hline die Möglichkeit zum Erfahrungsaustausch & 6 \\
$\begin{array}{l}\text { Praktische Beispiele und Demonstrationen zu den Themen } \\
\text { Pflege/Ergotherapie/ Physiotherapie }\end{array}$ & 4 \\
umfassende und verständliche Informationen zu den verschiedenen & 4 \\
Kursthemen & 3 \\
Informationen/Gespräche über Umgang mit psychischer Belastung & 2 \\
gute schriftliche Materialien & 2 \\
Zeit und Raum für Aussprache & 1 \\
die positive, wertschätzende Atmosphäre & \\
\hline
\end{tabular}

Anmerkung: Zum Teil wurden mehrere Aspekte von einem Teilnehmer aufgeführt, sodass die Gesamtsumme der Nennungen $\mathrm{N}=19$ übersteigt.

Zusammenfassend lässt sich aus der Evaluation des subjektiven Nutzens des Kurses durch die Angehörigen ersehen, dass insgesamt eine hohe Nutzerzufriedenheit gegeben war. Dies spiegelte sich auch in der Teilnahmefrequenz der einzelnen Kursabende wider. $75 \%$ der Teilnehmer nahmen an allen Kursterminen teil, $15 \%$ verpassten einen Kursabend und 10\% nahmen an zwei Kursabenden nicht teil. 


\section{Methode - Qualitative Untersuchung}

Das folgende Kapitel widmet sich der Methodik des qualitativen Untersuchungsteils. Zunächst werden die Stichprobe und der Untersuchungsablauf beschrieben. Daran schließt sich eine Darstellung der inhaltlichen und formalen Aspekte des LeitfadenInterviews an sowie eine Beschreibung des Interviewer-Trainings. Abschließend erfolgt eine Darstellung der Erfassung und Auswertung der Interviewtranskripte.

\subsection{Stichprobe}

Alle Teilnehmerinnen der qualitativen Studie nahmen auch an der quantitativen Studie teil. Für Einzelheiten zur Gewinnung und Zusammensetzung der Stichprobe sei auf Kapitel 4.3.1 verwiesen. Aus der Gesamtstichprobe von 85 Studienteilnehmern wurden aus konzeptionellen wie pragmatischen Erwägungen 14 Angehörige für die qualitative Untersuchung ausgewählt. Dabei wurden die quantitativen Daten als Kriterium für die Gewinnung der Stichprobe für die qualitative Untersuchung herangezogen. Wird bei vielen quantitativen Studien statistische Repräsentativität angestrebt, geht es bei qualitativen Untersuchungen darum, dass die Stichprobe den Forschungsgegenstand inhaltlich repräsentiert. Beim Ziehen der Stichprobe sollte maximale Variation erreicht werden (Mayring \& Gläser-Zikuda, 2005). Bei der Auswahl der Interviewpartnerinnen war deshalb darauf geachtet worden, dass gleichmäßig viele Angehörige ausgesucht wurden, die zum ersten Messzeitpunkt ein eher geringes Belastungsniveau (operationalisiert anhand der Depressions-, Angst- und körperliche Befindlichkeitsskalen) und solche, die ein hohes Belastungsniveau aufwiesen. Außerdem sollten in etwa gleich viele Angehörige der Treatment- als auch der Kontrollgruppe einbezogen werden. Die Interviews fanden zwischen 12 und 25 Monate nach dem ersten Messzeitpunkt (an dem der standardisierte Fragebogen im Rahmen der quantitativen Untersuchung ausgefüllt wurde) statt. Die ausgewählten Angehörigen (15 Ehefrauen) wurden zunächst angeschrieben und über die Ziele der qualitativen Untersuchung informiert und dann telefonisch kontaktiert um die Bereitschaft zur Teilnahme festzustellen. Nur eine der kontaktierten Angehörigen war aus zeitlichen Gründen nicht bereit, am Interview teilzunehmen. Somit wurden insgesamt 14 Interviews durchgeführt, wovon 3 Probeinterviews waren. Tabelle 11 liefert eine 
Übersicht über ausgewählte Merkmale der qualitativen Stichprobe.

Tabelle 11: Beschreibung der qualitativen Stichprobe anhand ausgewählter quantitativer Variablen

\begin{tabular}{cccccccccc}
\hline Nr. & Frau & Gruppe & $\begin{array}{c}\text { Alter } \\
\text { Ang. }\end{array}$ & $\begin{array}{c}\text { Alter } \\
\text { Pat. }\end{array}$ & $\begin{array}{c}\text { Pflege- } \\
\text { dauer } \\
\text { (Monate) } \\
\text { MZP 1 }\end{array}$ & $\begin{array}{c}\text { Pflege- } \\
\text { dauer } \\
\text { (Monate) } \\
\text { MZP 4 }\end{array}$ & $\begin{array}{c}\text { ADL } \\
\text { MZP 1 }\end{array}$ & $\begin{array}{c}\text { Aphasie } \\
\text { MZP 1 }\end{array}$ & $\begin{array}{c}\text { NP- } \\
\text { Defizite } \\
\text { MZP 1 }\end{array}$ \\
\hline 1 & & & & & & & & & \\
2 & W. & T & 58 & 57 & 4 & 29 & 60 & Ja & Ja \\
3 & A. & T & 72 & 76 & 2 & 14 & 60 & Nein & Ja \\
4 & K. & T & 59 & 70 & 2 & 14 & 25 & Nein & Ja \\
5 & T. & T & 56 & 75 & 36 & 52 & 35 & Nein & Nein \\
6 & M. & T & 64 & 62 & 2 & 15 & 95 & Ja & Ja \\
7 & So. & K & 60 & 61 & 52 & 74 & 85 & Nein & Ja \\
8 & F. & K & 59 & 58 & 35 & 57 & 35 & Nein & Nein \\
9 & Be. & K & 63 & 64 & 2 & 20 & 55 & Nein & Ja \\
10 & Sn. & K & 62 & 71 & 1 & 20 & 95 & Nein & Ja \\
11 & Br. & K & 48 & 66 & 2 & 16 & 95 & Nein & Ja \\
\hline
\end{tabular}

Anmerkungen: Gruppe: $\mathrm{T}=$ Treatmentgruppe, $\mathrm{K}=$ Kontrollgruppe; Alter Ang.: Alter des Angehörigen; Alter Pat.: Alter des Patienten; Pflegedauer MZP1 und MZP4: Zeit seit Schlaganfall in Monaten zum Messzeitpunkt 1 und Messzeitpunkt 4 (Zeitpunkt des Interviews); ADL MZP 1: Barthel-Index zum Messzeitpunkt 1; Aphasie: Aphasie zum Messzeitpunkt 1; NP-Defizite: Neuropsychologische Defizite in mindestens einem der 4 Bereiche Aufmerksamkeit, Gedächtnis, exekutive Funktionen und Abstraktionsvermögen zum Messzeitpunkt 1.

\subsection{Untersuchungsablauf}

Die Durchführung der Interviews fand in einem ruhigen Raum in der Tagesklinik statt. Vor Beginn des Interviews wurden die Teilnehmerinnen über die Ziele der Studie aufgeklärt und gebeten, eine Einverständniserklärung für die Aufzeichnung des Gesprächs auf Tonband zu unterschreiben, was alle Angehörigen ohne Vorbehalt taten. 


\subsection{Das Leitfaden-Interview und die erhobenen Variablen}

Die im qualitativen Teil der Untersuchung angewandte Methode lässt sich als teilstandardisiertes Leitfaden-Interview bezeichnen. Es enthält zwar obligatorische Fragevorgaben, lässt dem Interviewer aber Freiheiten bezüglich der Gestaltung der Fragenformulierung und -abfolge. Die Interviewerin wurde instruiert, die vorgegebenen Fragen gegebenenfalls durch klärende Nachfragen $\mathrm{zu}$ ergänzen und auf für die Fragestellung relevante Punkte einzugehen, die von den Befragten unabhängig vom Leitfaden angesprochen wurden (siehe dazu auch Abschnitt 7.4, Training der Interviewerin). Die wesentliche Abgrenzung $\mathrm{zu}$ standardisierten Interviews besteht darin, dass es im Leitfaden-Interview keine Antwortvorgaben gibt und die Befragten ihre Einstellungen und Erfahrungen frei formulieren können.

Bei der Gestaltung des Leitfadens flossen klare theoretische Vorannahmen und spezifische Überlegungen ein, was mit Mayrings (1995) Postulat einhergeht, dass sich das Vorgehen bereits bei der Erhebung an Theorie und Fragestellung orientieren sollte. Das Leitfaden-Interview explorierte in erster Linie das Bewältigungsverhalten der pflegenden Angehörigen. Berücksichtigt wurden dabei auch die Interaktionen innerhalb der Pflegedyade, die dynamische Entwicklung sowie der Kontext der Pflegebeziehung. Außerdem wurden die möglichen positiven Effekte durch den Angehörigenkurs untersucht. Der Leitfaden enthält im Einzelnen folgende Aspekte:

- Objektive Belastung durch die konkreten Anforderungen und Aufgaben der Pflege

- Coping-Strategien

- Soziale Unterstützung

- Frustrationen (Einschränkungen durch die Pflege und Unterstützung des Angehörigen)

- Gratifikationen/positive Aspekte der Pflege

- Beziehung zum Pflegebedürftigen

- Motivation zur Pflege

- Motivation zur Teilnahme am Angehörigenkurs bzw. Hinderungsgründe

- Wahrgenommene Effekte des Angehörigenkurses (nur Treatmentgruppe) 
Die alltäglichen Anforderungen und Aufgaben der Pflege wurden als Erzählanstoß zu Beginn des Interviews gewählt, da die Frage nach objektiven konkreten Sachverhalten als „Eisbrecher“ besonders geeignet erschien. Um ein möglichst differenziertes Bild der subjektiven Beurteilung der eigenen Pflegesituation zu erhalten, sah der Leitfaden vor, die korrespondierenden emotional-kognitiven Bewertungen der beschriebenen Situationen oder Sachverhalte näher zu explorieren (zum Beispiel: „Was geht Ihnen dabei durch den Kopf?“ „Was empfinden Sie dabei?“). Die offen formulierten Fragen sollten gewährleisten, dass auch neue oder individuelle Gesichtspunkte, die nicht in der Gestaltung des Leitfadens berücksichtigt worden waren, aufgenommen werden konnten, um ein vollständiges Bild der Pflegesituation zu erhalten. Der ausführliche Interviewleitfaden findet sich im Anhang B.

\subsection{Fähigkeiten und Training der Interviewerin}

\section{Anforderungen an die Interviewerin}

Die Datenerhebung anhand qualitativer Interviews ist nach Hopf (1991) mit anderen und zum Teil höheren Anforderungen an den Interviewer verbunden als die Erhebung mittels vollstandardisierter Fragebögen oder Testverfahren. Es herrscht in der Forschungsgemeinschaft eine relativ breite Übereinstimmung dahingehend, dass ,diese Interviews nur von Befragenden durchgeführt werden sollten, die verantwortlich in den jeweiligen Forschungsprojekten mitarbeiten oder die zumindest mit dem theoretischen Ansatz, den Fragestellungen und den Vorarbeiten des Projekts so vertraut sind, dass sie in der Lage sind, Interviews autonom zu führen“ (Hopf, 2003, S.358). Begründet wird dieses Postulat damit, dass der Befragende in einem nichtstandardisierten Interview imstande sein muss, „einzuschätzen, wann es inhaltlich angemessen ist, vom Frageleitfaden abzuweichen, an welchen Stellen es erforderlich ist, intensiver nachzufragen und [...] dem Befragten breite Artikulationschancen einzuräumen“ (Hopf, 2003, S.358).

Zusätzlich zu diesen inhaltlich-theoretischen Kompetenzen sollte der Interviewer auch kommunikative Qualifikationen vorweisen können, die jedoch wesentlich schwerer zu definieren sind. Einerseits erfordert die Durchführung von Interviews vom Interviewer sowohl direktive Fertigkeiten, andererseits auch eine eher passiv-rezeptive 
Haltung. Eine gute Balance zwischen diesen beiden Polen zu erreichen, ist wesentlich für den Erfolg (vgl. Hopf, 1991). Ein dominanter Kommunikationsstil mit häufigen Unterbrechungen des Befragten oder suggestiven Fragen und Vorgaben kann zur Folge haben, dass sich der Interviewte nicht als Experte ernst genommen fühlt und dem Interviewer mit Rückzug oder Reaktanz begegnet. Andererseits kann ein zu passives Gesprächsverhalten, bei dem der Befragte die Struktur vorgeben darf, dazu führen, dass relevante Aspekte nicht angesprochen werden oder für die Untersuchung eher unwesentliche Themen zu ausführlich behandelt werden. Ein zu starres Festhalten am Interviewleitfaden wiederum kann zur Folge haben, dass interessante, nicht antizipierte Gesichtspunkte verloren gehen (vgl. Hopf, 2003). Der Interviewer sollte idealerweise also fähig sein, eine offene, angenehme und entspannte Gesprächsatmosphäre herzustellen. Dabei sollte sich der Befragende auch an das Sprachniveau und -vermögen des Interviewpartners anpassen können (vgl. Lamnek, 1995).

\section{Training der Interviewerin}

Sämtliche Interviews wurden von einer studentischen Mitarbeiterin im 10. Semester des Psychologiestudiums durchgeführt. Effekte der sozialen Erwünschtheit, insbesondere hinsichtlich der Bewertung des Angehörigenkurses, die sicherlich stärker zum Tragen gekommen wären, wenn die Projektleiterin selbst die Interviews ausgeführt hätte, sollten dadurch abgemildert werden. Den Interviews ging eine intensive Schulung voraus, die folgende Aspekte umfasste:

- Vermittlung des theoretischen Hintergrundes und der Fragestellung der Studie;

- vertraut machen mit dem Leitfaden;

- allgemeine Hinweise zum Gesprächsverhalten (Paraphrasieren, Vertiefen emotionaler und kognitiver Aspekte, Anpassen an das Sprachniveau des Befragten, Vermeiden von Wertungen und suggestiven Vorgaben);

- Hinweise zum Umgang mit schwierigen Gesprächssituationen (z. B. redselige oder schweigsame Interviewpartner, Konfrontation mit Tränen und Trauer im Gesprächsverlauf);

- Analyse und Diskussion von „Kunstfehlern“ in der Gesprächsführung anhand konkreter Interviewprotokolle;

- Rollenspiele (mit Tonbandaufnahme). 
Drei Gespräche mit Angehörigen wurden als Probeinterviews geführt und gemeinsam mit der Interviewerin ausführlich besprochen. Diese Interviews gingen später nicht in die Auswertung ein. Auch alle weiteren Interviews wurden engmaschig von der Autorin supervidiert und mit der Gesprächsleiterin nachbereitet, dabei diente ein zweiseitiger Fragebogen als Leitfaden für die Nachbereitung (siehe Anhang B).

\subsection{Erfassung und Auswertung der Interviewtranskripte}

Alle Interviews wurden mit Tonband aufgenommen und von einer professionellen Schreibkraft transkribiert. Die Transkriptionsregeln sind Anhang B zu entnehmen. Mittels „Korrekturhören“ durch die Autorin und einen studentischen Mitarbeiter wurden sie von Übertragungsfehlern bereinigt. Die Interviewdauer betrug durchschnittlich 80 Minuten (Range 45 bis 90), was etwa 23 Seiten Transkription entspricht (Range 17 bis 26). Ausgewertet wurden insgesamt 11 vollständige Interviewtranskripte. Die Transkriptionsregeln sind im Anhang B aufgeführt.

Zur Auswertung des Interviews wurden folgende Materialien zusätzlich herangezogen:

- Eine Beschreibung der Interviewsituation mit der Gesprächspartnerin, etwaige bedeutsame Äußerungen vor oder nach dem Interview;

- eine stichwortartige Kurzbiographie;

- eine zusammenfassende Einschätzung der Validität der Interviewäußerungen anhand von Rating-Skalen durch die Interviewerin;

- eine ausführliche Falldarstellung.

Ein kritischer Punkt im Auswertungsprozess ist die Herstellung von Intersubjektivität. Qualitative Forschung zeichnet sich unter anderem dadurch aus, dass sich der Forscher bewusst als Person in den Forschungsprozess einbringt. Dies hat jedoch die Notwendigkeit der kritischen Reflexion der eigenen Rolle zur Folge, um „blinde Flecken“ zu vermeiden. Dazu empfehlen Corbin und Strauss (1990) ausdrücklich den kollegialen Austausch in Forschungsprozess.

Im Rahmen des hier vorgestellten Projekts konnten zwei Personen dafür gewonnen werden: Ein Referendar im Lehramt (Geschichte und Religion), der eine eher 
alltagspsychologische Perspektive mitbrachte, las alle Transkripte und diskutierte die Falldarstellungen mit der Autorin. Zusätzlich lieferte ein Neuropsychologe, der selbst im Bereich der Angehörigenforschung wissenschaftlich gearbeitet hat, ergänzende fachspezifische Hinweise zu einem Teil der Interviews. Der Austausch mit den externen Intervisoren diente als kritisches Korrektiv mit dem Ziel, Hypothesen und Bewertungen zu hinterfragen (vgl. Lämmler, 1998).

\section{Inhaltsanalytische Auswertung der Interviewtranskripte}

Im Folgenden wird der Ablauf der inhaltsanalytischen Auswertung beschrieben. Das Vorgehen zur Festlegung des Kategorienschemas orientierte sich an der Strategie der inhaltlichen Strukturierung nach Mayring (1995; vgl. auch Schmidt, 2003). Dieses umfasste fünf Schritte (Schmidt, 2003):

1. Materialorientierte Bildung von Auswertungskategorien

2. Zusammenstellung der Auswertungskategorien zu einem Kodierleitfaden

3. Kodierung des Materials

4. Quantifizierende Materialübersichten

5. Vertiefende Fallinterpretationen

Materialorientierte Bildung von Auswertungskategorien. Zunächst galt es, eine materialorientierte Bildung von Auswertungskategorien vorzunehmen. In diesem ersten Schritt wurden die Interviews noch nicht vergleichend betrachtet. Ziel war es, für jedes einzelne Interviewtranskript die zugrundeliegenden Bewältigungsstrategien und weitere relevante Einzelaspekte herauszuarbeiten.

Erstellung eines Kodierleitfadens. Basierend auf den gefundenen Bewältigungsstrategien wurden dann in einem zweiten Schritt die Entwürfe der Auswertungskategorien zu einem Kodierleitfaden zusammengefasst. Den einzelnen Kategorien wurden ausführliche Beschreibungen zugeordnet.

Erster Materialdurchlauf. Mit Hilfe des Kodierleitfadens wurde in einem dritten Schritt das erhobene Material kodiert, d. h. es wurden entsprechende Textpassagen eines Interviews einer Kategorie zugeordnet. Die Brauchbarkeit des Kodierleitfadens wurde zunächst an fünf Interviews erprobt und bewertet. Einige Kategorien wurden daraufhin modifiziert bzw. ganz aus dem Leitfaden genommen. 
Kodierung des Materials. Unter Verwendung des modifizierten Kodierleitfadens wurden dann alle Interviews eingeschätzt und klassifiziert. Die Kategorien, die im vorangegangenen Auswertungsschritt aus dem Material heraus gebildet wurden, wurden nun auf das Material angewendet. Auf die Verwendung eines speziellen Computergestützten Auswertungsprogramms wurde verzichtet, es wurde lediglich zur Unterstützung ein Textverarbeitungsprogramm eingesetzt.

Quantifizierende Materialübersichten. Schließlich wurden die Ergebnisse der Kodierung quantifizierend zusammengestellt. Dabei wurden Häufigkeitsangaben zu den einzelnen Auswertungskategorien gemacht, die einen ersten Überblick über Verteilungen im Material gaben und Informationen zur „Datenbasis“ lieferten.

Vertiefende Fallinterpretationen. Auf dieser Grundlage konnten Hypothesen am Einzelfall überprüft werden und begriffliche Konzepte ausdifferenziert werden. Mit den durch die Kodierung ermittelten Konstellationen wurden dann Fälle für eine vertiefende Analyse ausgewählt, wobei auch die Daten aus dem quantitativen Untersuchungsteil herangezogen wurden.

\section{Festlegung der Auswertungskategorien}

Grundlage für die Erstellung des Kategoriensystems zur Auswertung der Interviews war das von Lämmler (1998) entwickelte Bewältigungsinventar (für eine zusammenfassende Darstellung der Studie von Lämmler siehe Kapitel 2.6.4). Tabelle 12 enthält das endgültige Kategoriensystem, das aus dem Interviewmaterial der vorliegenden Untersuchung gewonnen wurde. Die einzelnen Kategorien wurden dabei Lämmler (1998) folgend zwei grundsätzlichen Dimensionen zugeordnet: Bewältigungsstrategien, die entweder 1) in erster Linie der Verbesserung des Zustandes des Patienten (patientenzentriert) oder 2) der Verbesserung der eigenen Befindlichkeit (ichzentriert) dienen. 
Tabelle 12: Kategoriensystem der Bewältigungsstrategien

\begin{tabular}{|c|c|}
\hline $\begin{array}{l}\text { Patientenzentrierte } \\
\text { Bewältigungsstrategie }\end{array}$ & Definition \\
\hline Informationssuche & $\begin{array}{l}\text { Aktive Suche nach Informationen mit dem Ziel, besser mit der } \\
\text { Krankheit und deren Folgen umgehen zu können. Dies kann die } \\
\text { Suche nach entsprechender Literatur beinhalten, die Teilnahme am } \\
\text { Angehörigenkurs oder auch die Initiierung von Gesprächen mit } \\
\text { Ärzten und Therapeuten. }\end{array}$ \\
\hline $\begin{array}{l}\text { Fernhalten psychischer } \\
\text { Belastungen }\end{array}$ & $\begin{array}{l}\text { Zielt darauf ab, unangenehme oder beängstigende Sachverhalte und } \\
\text { Situationen vom Patienten fernzuhalten. Das kann bedeuten, dass } \\
\text { die realen Verhältnisse bewusst verschwiegen werden oder dem } \\
\text { Patienten eine (vermeintlich) belastende Situation, wie z. B. der } \\
\text { Besuch einer Kurzzeitpflegeeinrichtung, nicht zugemutet wird. }\end{array}$ \\
\hline $\begin{array}{l}\text { Anpassung der } \\
\text { Lebensumwelt }\end{array}$ & $\begin{array}{l}\text { Umfasst Veränderungen der Lebensumwelt mit dem Ziel der } \\
\text { leichteren Bewältigung des Alltags und einer größeren } \\
\text { Selbständigkeit des Patienten. Dazu gehören der Einsatz von } \\
\text { Hilfsmitteln (z. B. Badewannenlifter) im Haushalt oder } \\
\text { Umbaumaßnahmen, wie z. B. die Entfernung von Schwellen. }\end{array}$ \\
\hline Zuspruch & $\begin{array}{l}\text { Zielt darauf ab, die emotionale Situation und Motivation des } \\
\text { Pflegebedürftigen zu stabilisieren bzw. zu verbessern. Dies } \\
\text { geschieht durch die Verdeutlichung der bisherigen Fortschritte, } \\
\text { Vergleiche mit anderen oder den Einsatz von Belohnungen. }\end{array}$ \\
\hline $\begin{array}{l}\text { Sicherstellen } \\
\text { optimaler Behandlungs- } \\
\text { bedingungen }\end{array}$ & $\begin{array}{l}\text { Bezieht sich auf alle Bemühungen, für den Patienten die optimale } \\
\text { Therapie zu erreichen. Dies kann z. B. beinhalten: eine bestimmte } \\
\text { Therapie bei den Ärzten oder eine stationäre Rehabilitation bei der } \\
\text { Krankenkasse durchsetzen oder für eine ambulante Therapie einen } \\
\text { organisatorischen und finanziellen Mehraufwand in Kauf nehmen. }\end{array}$ \\
\hline $\begin{array}{l}\text { Förderung der } \\
\text { Selbständigkeit }\end{array}$ & $\begin{array}{l}\text { Umfasst alle Maßnahmen, selbständiges Verhalten des Patienten zu } \\
\text { unterstützen, z. B. den Patienten die Körperpflege selbständig } \\
\text { verrichten oder ihn alleine öffentliche Verkehrsmittel benutzen zu } \\
\text { lassen. }\end{array}$ \\
\hline Kontrolle & $\begin{array}{l}\text { Interventionen, mit denen der pflegende Angehörige versucht, } \\
\text { Kontrolle gegenüber dem Erkrankten auszuüben, um inadäquates } \\
\text { oder vermeintlich inadäquates Verhalten zu verhindern. Dies } \\
\text { können z. B. Bemühungen sein, das Autofahren zu verhindern oder } \\
\text { den Bewegungsradius des Patienten einzuschränken. }\end{array}$ \\
\hline $\begin{array}{l}\text { Co-therapeutisches } \\
\text { Engagement }\end{array}$ & $\begin{array}{l}\text { Aktivitäten, die nicht im engeren Sinne pflegerische Tätigkeiten } \\
\text { darstellen, sondern einen therapeutischen Charakter haben und } \\
\text { bewusst eine Verbesserung der Einschränkungen des Patienten } \\
\text { herbeiführen sollen, z. B.: Behindertenschwimmen, Lagerung } \\
\text { des Patienten nach Bobath, Ermunterung zum Besuch einer } \\
\text { Aphasikergruppe, Üben von Fertigkeiten wie Rechnen, Lesen } \\
\text { oder Sprechen, Gedächtnis- und Konzentrationsübungen. }\end{array}$ \\
\hline
\end{tabular}


Tabelle 12 Fortsetzung

\begin{tabular}{|c|c|}
\hline $\begin{array}{l}\text { Patientenzentrierte } \\
\text { Bewältigungsstrategie }\end{array}$ & Definition \\
\hline $\begin{array}{l}\text { Stärkung des } \\
\text { Kompetenzerlebens }\end{array}$ & $\begin{array}{l}\text { Zielt darauf ab, die emotionale Befindlichkeit des Patienten zu } \\
\text { verbessern. Dies geschieht über die bewusste Einbeziehung der } \\
\text { Pflegebedürftigen bei Alltagsangelegenheiten oder Problemen. } \\
\text { Dabei werden gezielt (frühere) Kompetenzen des Patienten } \\
\text { angesprochen. Damit soll gegenüber dem Pflegebedürftigen } \\
\text { verdeckt werden, dass die Erkrankung möglicherweise einen } \\
\text { Kompetenzverlust mit sich gebracht hat. }\end{array}$ \\
\hline $\begin{array}{l}\text { Ichzentrierte } \\
\text { Bewältigungsstrategie }\end{array}$ & Definition \\
\hline Sozialer Vergleich & $\begin{array}{l}\text { Dient dazu, das eigene Schicksal zu relativieren und somit eine } \\
\text { emotionale Entlastung herbeizuführen. Durch den sozialen } \\
\text { Vergleich wird versucht, eine positivere Bewertung der eigenen } \\
\text { Situation und eine Verifizierung des eigenen Umgangs mit der } \\
\text { Erkrankung des Angehörigen zu erreichen. }\end{array}$ \\
\hline Temporaler Vergleich & $\begin{array}{l}\text { Dient ebenfalls dazu, bestehende Einschränkungen der } \\
\text { Lebenssituation } \mathrm{zu} \text { relativieren und eine emotionale } \\
\text { Stabilisierung } \mathrm{zu} \text { erreichen. Durch temporale Vergleiche } \\
\text { versucht der Angehörige, zu einer positiveren Bewertung der } \\
\text { Lebenssituation zu kommen. }\end{array}$ \\
\hline Akzeptieren & $\begin{array}{l}\text { Umfasst alle Aussagen, die ein Akzeptieren der Krankheit und } \\
\text { ihrer Folgen erkennen lassen. Damit einher geht auch eine } \\
\text { grundsätzlich realistische Einschätzung der Erkrankung und der } \\
\text { noch zu erwartenden Verbesserungen. }\end{array}$ \\
\hline Gutes im Negativen sehen & $\begin{array}{l}\text { Kognitive Mechanismen, die eine positive Umbewertung } \\
\text { einzelner Aspekte der Lebenssituation herbeiführen oder auch } \\
\text { positive Bereiche hervorheben. }\end{array}$ \\
\hline Distanzierung & $\begin{array}{l}\text { Zielt darauf ab, Ängste in Bezug auf die Lebenssituation bzw. } \\
\text { die weitere Entwicklung der Erkrankung des Angehörigen } \\
\text { abzuwehren. Dies kann in unterschiedlichem Ausmaß reflektiert } \\
\text { sein. }\end{array}$ \\
\hline $\begin{array}{l}\text { Vergegenwärtigen } \\
\text { der eigenen Erfolge }\end{array}$ & $\begin{array}{l}\text { Beinhaltet kognitive Prozesse, bei denen sich die Pflegeperson } \\
\text { bisherige Erfolge im Umgang mit dem kritischen Lebensereignis } \\
\text { „Schlaganfall“ bewusst macht und damit zur Stärkung des } \\
\text { eigenen Selbstwertgefühls und Kompetenzerlebens beiträgt. }\end{array}$ \\
\hline $\begin{array}{l}\text { Inanspruchnahme } \\
\text { sozialer Unterstützung }\end{array}$ & $\begin{array}{l}\text { Aktive Bemühungen, soziale Unterstützung in Anspruch zu } \\
\text { nehmen, sei es in emotionaler oder in instrumenteller Form. }\end{array}$ \\
\hline
\end{tabular}


Tabelle 12 Fortsetzung

\begin{tabular}{|l|l|}
\hline $\begin{array}{l}\text { Ichzentrierte } \\
\text { Bewältigungsstrategie }\end{array}$ & Definition \\
\hline $\begin{array}{l}\text { Inanspruchnahme } \\
\text { professioneller } \\
\text { Unterstützung }\end{array}$ & $\begin{array}{l}\text { Aktive Bemühungen, professionelle Unterstützung zu nutzen, } \\
\text { z. B. Inanspruchnahme eines Pflegedienstes, einer } \\
\text { Kurzzeitpflegeeinrichtung oder eines Besuchsdienstes. }\end{array}$ \\
\hline Erholung & $\begin{array}{l}\text { Verhaltensweisen, die einen Ausgleich zur körperlichen und } \\
\text { seelischen Belastung der Pflege bilden. Dies kann eine passive } \\
\text { oder aktive Form annehmen (z. B. regelmäßiger Mittagsschlaf } \\
\text { vs. Sport treiben). }\end{array}$ \\
\hline $\begin{array}{l}\text { Pflege der } \\
\text { eigenen Bedürfnisse }\end{array}$ & $\begin{array}{l}\text { Über die Sorge für Erholung hinausgehende Bemühungen, sich } \\
\text { neben der Pflegetätigkeit Zeit für andere Interessen und Hobbies } \\
\text { zu nehmen und dies auch gegebenenfalls gegenüber dem } \\
\text { Pflegebedürftigen durchzusetzen. Damit einher geht ein } \\
\text { Selbstkonzept, in dem die Pflegetätigkeit nicht zum zentralen } \\
\text { Aspekt erhoben wird. }\end{array}$ \\
\hline Emotionsausdruck & $\begin{array}{l}\text { Verhaltensweisen, die zu einer emotionalen „Reinigung“ führen, } \\
\text { wie Schimpfen oder Weinen. }\end{array}$ \\
\hline Substanzgebrauch & $\begin{array}{l}\text { Die Einnahme von Substanzen wie Beruhigungsmittel, } \\
\text { Schlafmittel oder Alkohol, mit dem Ziel sich zeitweilig zu } \\
\text { betäuben. }\end{array}$ \\
\hline Religiöse Bewältigung & $\begin{array}{l}\text { Religiöse Überzeugungen, die Trost spenden bzw. den Umgang } \\
\text { mit der Erkrankung erleichtern. }\end{array}$ \\
\hline
\end{tabular}

\section{Anmerkungen zu den gefundenen Kategorien und zu den Klassifikationskriterien „patientenzentriert“ vs. „ichzentriert“}

Zum überwiegenden Teil stimmt das obige Bewältigungsinventar mit dem von Lämmler gefundenen überein, bei den ichzentrierten Strategien kamen jedoch einige Kategorien hinzu (i. e. temporaler Vergleich, Inanspruchnahme professioneller Unterstützung, Emotionsausdruck und Substanzgebrauch). Die Kategorie „Gutes im Negativen sehen“ beinhaltet die von Lämmler (1998) benannte Strategie „positive Umdeutung“, geht jedoch noch darüber hinaus. Es werden nicht nur kognitive Mechanismen darunter gefasst, die auf eine Umdeutung der Situation insgesamt oder in Teilaspekten abzielen, sondern auch Bemühungen, auf positive Aspekte der Lebenssituation zu fokussieren (z. B. „,immerhin kann ich mich noch mit meinem Mann unterhalten“). Die zu den 
patientenzentrierten Strategien zählende Kategorie „Co-therapeutisches Engagement“ wurde ebenfalls neu aufgenommen. Dafür wurde die von Lämmler gefundene Kategorie „pflegerisches Engagement“ nicht berücksichtigt, da pflegerische Tätigkeiten in der vorliegenden Stichprobe bereits umfassend im quantitativen Untersuchungsteil aufgenommen wurden. Dem aufmerksamen Leser mag die Strategie „Cotherapeutisches Engagement“ identisch mit der Strategie „Selbständigkeit des Patienten fördern" erscheinen. In der Tat dienten co-therapeutische Bemühungen zumeist langfristig dazu, die Unabhängigkeit des Patienten zu unterstützen. Da diese beiden Bewältigungsformen jedoch ein unterschiedliches Ausmaß an Aktivität von Seiten des pflegenden Angehörigen erforderte, wurden sie getrennt klassifiziert. Wurde der Patient dazu angehalten, eine Aufgabe oder Situation allein zu bewältigen, dann wurde dies als „Selbständigkeit des Patienten fördern“ kodiert. War jedoch die Angehörige bei der geschilderten Situation in irgendeiner Weise aktiv beteiligt (z. B. mit dem Patienten rechnen üben, Patienten zum Mittagsschlaf nach den Bobath-Prinzipien lagern), dann galt dies als „Co-therapeutisches Engagement“.

Abschließend sind noch einige Anmerkungen zu der Einordnung der jeweiligen Kategorien in die Dimensionen patientenzentriert und ichzentriert angezeigt. Der kritische Leser wird die Einteilung einiger Kategorien wie „Informationssuche“, „Anpassung der Lebensumwelt" und „Suche nach sozialer Unterstützung“ in die eine oder andere Dimension möglicherweise als willkürlich empfinden (siehe auch Lämmler, 1998). Eine Zuordnung dieser Bewältigungsstrategien zur jeweils anderen Dimension ist tatsächlich denkbar. Die in dieser Arbeit vorgenommene Einteilung folgte jedoch der vordergründigen Zielsetzung der gezeigten Bewältigungsform. Die Suche nach Informationen und die Anpassung der Lebensumwelt hatten in der untersuchten Stichprobe überwiegend eine Befindlichkeitsverbesserung des Patienten zum Ziel, während die Inanspruchnahme sozialer Unterstützung vor allem die Situation der Angehörigen selbst verbessern sollte. Nichtsdestoweniger sollten die von der vordergründigen Motivation möglicherweise verdeckten sekundären Motive nicht übersehen werden. So kann z. B. hinter der Förderung der Selbständigkeit des Patienten das sekundäre Motiv stehen, sich selbst zu entlasten und größere persönliche Freiheit zu gewinnen. Genauso kann sich hinter co-therapeutischem Engagement das Motiv 
verbergen, Schuldgefühle zu verringern. Hauptziel co-therapeutischer Bemühungen wäre also in diesem Fall die Verbesserung der eigenen Befindlichkeit (siehe auch Lämmler, 1998). 


\section{Ergebnisse - Qualitative Untersuchung}

Die nachfolgende Darstellung der Befunde der qualitativen Untersuchung umfasst drei Teile. Im ersten Abschnitt (8.1) erfolgt eine Darstellung der Häufigkeiten der verwendeten Strategien verbunden mit einer inhaltlichen Erläuterung der ermittelten Bewältigungsstrategien. Mit Hilfe ausgewählter Zitate aus den Interviewtranskripten werden die einzelnen Bewältigungsstrategien exemplarisch illustriert, sodass ein tiefergehendes Verständnis von den Bewältigungsversuchen pflegender Angehöriger möglich wird. Im zweiten Teil (8.2) wird eine Gesamtbeurteilung des Bewältigungsverhaltens jeder einzelnen pflegenden Angehörigen aus der qualitativen Stichprobe auf der Grundlage der verwendeten Bewältigungsstrategien vorgenommen. Der dritte Abschnitt (8.3) greift schließlich zwei Fälle heraus, die vertiefend unter Einbeziehung der quantitativen Daten analysiert werden.

\subsection{Die ermittelten Bewältigungsstrategien}

Wie schon weiter oben beschrieben dienen patientenzentrierte Bewältigungsstrategien primär dazu, eine Verbesserung des Zustandes des Patienten zu erreichen. In der hier befragten Stichprobe fanden sich neun verschiedene Untergruppen dieser Bewältigungsdimension. Ichzentrierte Bewältigungsstrategien zielen vor allem darauf $\mathrm{ab}$, eine Verbesserung der eigenen Befindlichkeit zu erreichen. Insgesamt konnten in der vorliegenden Stichprobe dreizehn verschiedene Kategorien gefunden werden, die dieser Dimension zuzuordnen waren. Tabelle 13 ist $\mathrm{zu}$ entnehmen wie sich die Häufigkeitsverteilung darstellt. 
Tabelle 13: Häufigkeit der Nennungen der verwendeten Bewältigungsstrategien

Einschätzungsdimension

\section{Trifft zu}

$\% \quad \mathrm{~N}$

Patientenzentrierte Bewältigungsstrategien

Informationssuche

$90.9 \quad 10$

Fernhalten psychischer Belastungen

81.8

72.7

9

Anpassung der Lebensumwelt

63.6

8

Zuspruch

Sicherstellen optimaler Behandlungsbedingungen

$63.6 \quad 7$

Förderung der Selbständigkeit

54.5

45.5

6

Kontrolle

Co-therapeutisches Engagement

27.3

5

Stärkung des Kompetenzerlebens

$27.3 \quad 3$

\section{Ichzentrierte Bewältigungsstrategien}

Sozialer Vergleich

$90.9 \quad 10$

Temporaler Vergleich

90.9

9

Akzeptieren

$81.8 \quad 9$

Gutes im Negativen sehen

Distanzierung

$72.7 \quad 8$

Vergegenwärtigen der eigenen Erfolge

$\begin{array}{ll}72.7 & 8\end{array}$

Inanspruchnahme sozialer Unterstützung

72.7

8

Erholung

63.6

54.5

Pflege der eigenen Bedürfnisse

Inanspruchnahme professioneller Unterstützung

Emotionsausdruck

$18.2 \quad 2$

\section{Patientenzentrierte Bewältigungsstrategien}

Informationssuche. Wie aus Tabelle 13 ersichtlich ist die Suche nach Informationen die am häufigsten verwendete patientenzentrierte Bewältigungsstrategie. Für die Teilnehmerinnen am Angehörigenkurs war das Bedürfnis nach Informationen ein wichtiger Motivationsgrund, den Kurs zu besuchen, so auch für Frau M.:

Interviewerin: Sie haben von sich selbst aus auch schon den Angehörigenkurs angesprochen. Wissen Sie noch, was Sie motiviert hatte, am Angehörigenkurs teilzunehmen? 
Frau M.: Ja. Ja also, des kann ich fast genau sagen: Hilfestellung ganz einfach. Aber gar nich jetzt mal im übertragenen Sinne, sondern bei den ganz praktischen Dingen, ja. Also, ich hab gedacht, des kann auf gar keinen Fall verkehrt sein: Du weißt gar nicht, wie's weiter geht, du musst damit umgehn können. Und ich hab ja denn auch gemerkt, es wurde alles angesprochen, wie setz ich meinen Angehörigen vom Bett in den Stuhl/in den Rollstuhl und umgekehrt. Wie kann ich ihm beim Waschen behilflich sein, wie kann ich behilflich sein, wenn er sich nicht anziehn kann. (...)(Z. 668-676)

Angehörige, die sich gegen eine Teilnahme entschieden hatten, führten als einen Hinderungsgrund häufig an, dass ihr Informationsbedürfnis schon ausreichend durch das Lesen entsprechender Literatur gedeckt sei:

Interviewerin: Sie erzählten vorhin, dass Sie sich selbst auch etwas angelesen haben.

Frau Sn.: Ja, erarbeitet so'n bisschen, nich. Die Sachen, die so draußen liegen, ja. Nich, in den Krankenhäusern, da gibt's denn ja meistens so kleine Broschüren. Und einmal hat die Apotheke was verteilt. Äh, dann gibt es dieses Gesundheitsheftchen von der Apotheke, dann gibt's das Seniorenheft von der Apotheke. Und da stehen ja des öfteren alles solche Sachen drin. Und da liest man dann drin, ja. Und dann hatten wa sogar irgendwo mal was zugeschickt gekriegt, ich weiß gar nicht, wo das herkam, das waren auch so'ne kleine Hand...schriften mit so zehn Seiten, ja - Schlaganfall: Wie ist das dann, in der Beziehung usw. Und ich muss sagen, das reicht auch dann, was ich // was man an // lesen kann - im Hausgebrauch. (...)(Z. 687-695)

Fernhalten psychischer Belastungen. Das Fernhalten belastender Sachverhalte stellt in dieser Stichprobe ebenfalls eine sehr beliebte Strategie dar, die vom überwiegenden Teil der Angehörigen verwendet wurde. Diese kann unterschiedliche Formen annehmen; so verschweigen viele Angehörige dem Pflegebedürftigen die eigene Belastung, um ihn nicht zu beunruhigen:

Frau M.: (...) Man nimmt sich ja so zusammen, man ist ja so konzentriert. Also, der Angehörige darf nich merken, wenn's einem mal nicht so richtig wohl zumute ist. Dann kann ich ihm nicht die Kraft geben, die er braucht. (...) (Z. 689-691)

In einigen Fällen gehen die Bemühungen, den Patienten zu schonen, sogar so weit, dass eigene gesundheitliche Probleme ignoriert werden, wie bei Frau F., die einen Operationstermin aufschiebt, um es ihrem Mann $\mathrm{zu}$ ersparen, in eine Kurzzeitpflegeeinrichtung zu gehen: 
Frau F.: (...) dann muss ich auch selber zum Doktor, da hab ich jetzt auch'n Problem, ich weiß nicht, ob die Möglichkeit besteht, dass ich ins Krankenhaus muss, ob es da 'ne Möglichkeit gibt, dass ich ihn mitnehmen kann, denn wenn er woanders hinkommt, das geht nicht gut, wenn er dann nur Fremde um sich hat oder wenn es Menschen sind, die vielleicht noch schlimmer dran sind oder die dann immer so'n bisschen bösartig sind, das packt er nicht mehr. (Z. 122-127)

Dass die Bewältigungsstrategie „Fernhalten psychischer Belastungen“ mitunter sehr zu Lasten der Angehörigen gehen und zusätzliche Einschränkungen wie soziale Isolation nach sich ziehen kann, veranschaulicht folgendes Beispiel besonders deutlich:

Frau A.: (...) Das ist also das, was mich am meisten bedrückt: Wir haben keine Gespräche mehr, ich erzähl ihm Zeug, ich erzähl ihm, die Nachbarin hat das und das gemacht oder der Junge hat sich das Bein gebrochen, von nebenan, oder irgendsolche Geschichten, aber wir können keine richtigen Gespräche führen. Ich vermeide auch alles, was ihn ... aufpulvern könnte. Also, wenn ich jetzt // also, wir haben zum Beispiel (LACHEN) politisch unterschiedliche Meinungen. Wenn ich also jetzt von meiner Partei was sage, und da würda lospulvern. Und weshalb soll ich ihm diese Aufregung verschaffen? Denn das bekommt ihm gar nicht gut, gar nicht. Er kann überhaupt keine Aufregung vertragen. Es darf eigentlich niemand zu uns kommen außer unseren Kindern und Enkelkindern, das geht noch, aber ist da gleich große Unruhe, er macht dann in die Hose und alle solche Geschichten. (...)(Z. 35-44)

Anpassung der Lebensumwelt. Auch Veränderungen der Lebensumwelt werden von vielen pflegenden Angehörigen der vorliegenden Stichprobe vorgenommen, um den Alltag für den Patienten und somit auch für den Angehörigen einfacher zu gestalten. Manche Maßnahmen erfordern eher weniger Aufwand wie im Beispiel von Frau So., deren Mann unter einem starken Gesichtsfeldausfall leidet:

Frau So.: (...) Er weiß nie, welcher Tag ist, ja. Mit der Uhr hat man sich ja helfen können, indem man 'ne sprechende Uhr kauft, und dann er selbst erfragen kann. (...)(Z. 45-46)

Die Anpassung der Lebensumwelt kann aber auch einen größeren Aufwand beinhalten, wie z. B. der Umzug in eine Wohnung, die den Erfordernissen der Pflegebedürftigkeit besser gerecht wird:

Frau A.: (...) Also ich möchte ihn solange wie möglich zu Hause behalten. Wir haben 'ne sehr bequeme, 'ne sehr angenehme Wohnung. Wir haben früher ein 
Haus bewohnt und vor fünf Jahren, als es meinem Mann anfing, schlechter zu gehen, ham wa das aufgegeben und es wurde in derselben Straße gegenüber eine sehr schöne große 3-Zimmer-Wohnung im Erdgeschoß frei. In einem Mehrfamilienhaus, und da dacht ich, das machen wa jetzt. (...) und da wohnen wir, sehr behaglich. Erdgeschoss, wie gesagt, nicht viel Treppen zu steigen, sind keine Schwellen, schöner Balkon, also es ist 'ne // man kann ruhig sagen, 'ne komfortable Wohnung. Und äh, das können wa meistern, also da komm wa zurecht. (Z. 138-146)

Zuspruch. Wenn Angehörige die Bewältigungsstrategie „Zuspruch“ anwenden, zielen sie darauf ab, die Stimmung des Patienten zu verbessern. Dass die Bemühungen, die Motivationslage des Patienten zu stärken, aber nicht immer sanfter Natur sein müssen, zeigt folgendes Beispiel:

Frau Be.: Dann setzt er sich hin und erzählt mir: Ja, bin ein Krüppel, bin zu nichts nutze. So diese schlimmen Sachen. Also, da fällt er ins Loch, fällt er ins Tief ... also das wa denn wirklich alle mit vereinten Kräften ihn da wieder rausholen müssen.

Interviewerin: Und wie ist das für Sie, wenn er sowas sagt?

Frau Be.: Schlimm, ja. Also ... es kommt immer drauf an. Manchmal, denn dann umhüll ich ihn und hüll ihn ein. Gut, wird nicht besser. Und dann werd ich energisch und brüll ihn manchmal an und sage: Du bist ungerecht. Guck mal an, was du alles kannst, wie's dir geht. Deine Ärztinnen sind mit dir zufrieden, deine Therapeutin und du machst jetzt hier diese Schau und dieses Theater. Und dann guckt er mich an und sagt er zu mir: So schlimm? „Noch schlimmer!“. Und denn kriegen wa ihn wieder raus aus seinem Loch. Gestern, wir sind im schönsten Sonnenschein auf'm Balkon: „Ja, und wenn es so bleibt und so weiter geht?“ Sag ich: „Was machste dann? Springste vom Balkon?“ Wissense, ich muss dann manchmal so, hört sich dann zwar hart an, aber ... „Nee“, sachta, ,eene Treppe lohnt ja nich.“(Z. 99-113)

Sicherstellen optimaler Behandlungsbedingungen. Angehörige nehmen häufig große Unannehmlichkeiten in Kauf, wenn sie die Therapiebedingungen als nicht optimal einschätzen. Um eine Verbesserung zu erreichen, scheuen sich manche Angehörige nicht, den Arzt zu wechseln oder große Wege zurückzulegen, um es dem Patienten zu ermöglichen ein spezielles Therapieangebot zu nutzen. Zum Teil schrecken sie auch nicht vor Auseinandersetzungen mit Klinikpersonal oder mit der Krankenkasse zurück, um die Behandlung zu optimieren, wie die folgenden Beispiele zeigen:

Frau K.: (...) und dann hat er im Krankenhaus am Po 'nen Pilz gekriegt an jeder Seite. Also des heißt, mangelnde Hygiene, das iss natürlich schlimm. Also, da 
hab ich mich so aufgeregt, dass die schon froh waren, als der raus ging. Ich hab SO EIN Theater gemacht und jedes Mal hab ich gesagt: Hamse die Salbe mit? Hamse die Salbe mit? (...)(Z. 525-529)

Frau Be.: (...) ick hab ja gekämpft denn wie 'ne Löwin, dass er hier in die Tagesreha kommt und nicht ins Haus Berlin. Da wär' mein Mann geflitzt, der hätt sich 'n Taxi bestellt und wär nach Hause gekommen. Der wäre nich noch mal da $2 \frac{1}{2}$ Monate geblieben, wo auch nicht mehr passiert als hier. (...)(Z. 699701)

Förderung der Selbständigkeit. Eine bewusste Förderung der Selbständigkeit der Patienten wird lediglich von der Hälfte der hier untersuchten Angehörigen als Bewältigungsstrategie eingesetzt. Frau G., deren Mann unter einer schweren Aphasie leidet, legt viel Wert darauf, dass ihr Mann Sprechen trainiert und setzt ihn immer wieder Situationen aus, in denen er gezwungen ist, selbständig zu kommunizieren:

Frau G.: Wir haben so 'ne Angewohnheit, wir bestellen immer von Freitag auf Sonnabend unsere Quiddeln [?] bei 'ner Freundin, die ist Bäckermeisterin, und wenn wir mal nicht da waren oder vergessen haben und nicht gleich bezahlt haben, dann bringense immer Schrippen oder rufen an. Wennse mal nich anruft, dann drängelt er: H., Brötchen. Dann sag ich: Dann ruf doch mal an, die Nummer weißte, wose steht, und nu ruf mal an. Und dann hat er's gemacht und hat eben die Brötchen bestellt. (Z. 393-397)

Dass die Berufstätigkeit der Angehörigen ein begünstigender Faktor für die Förderung der Selbständigkeit des Patienten ist, zeigt das Beispiel von Frau Br., die als einzige in der Stichprobe des qualitativen Untersuchungsteils noch einen Beruf ausübt.

Frau Br.: Diese Situation als mein Mann wieder zu Hause war, war die: Ich bin weiter Arbeiten gegangen, ich hab mir keinen Tag freigenommen und er musste sich behelfen, was er dann auch konnte. Ich hab ihm das zwar vorbereitet, dann hatte er mich jeden Tag um eine bestimmte Zeit anzurufen - praktisch wie man's mit 'nem Kind macht, was man erstmalig alleine zu Hause läßt -, ob auch alles jeklappt hat mit'm Spritzen und mit'm Essen entsprechend, ja. Als es dann auch geklappt hat, ham wa's dann auch gelassen, hab ich gesagt: Nun lassen wa's, brauchst nicht mehr anzurufen. Aber diese Situation war nich so gewesen, dass ich da Probleme mit hatte, da er immer pünktlich angerufen hat. Hätte er nicht angerufen, hätte ich'n Problem gehabt, dann hätt' ich nach Hause gemusst um zu gucken, was los ist. Aber das ergab sich nicht. (Z. 213-222) 
Zwar übt Frau Br. in der Anfangszeit noch Kontrolle aus, indem ihr Mann sie zu bestimmten Zeiten bei der Arbeit anrufen soll, mit zunehmender Selbständigkeit nimmt sie diese Kontrolle jedoch wieder zurück.

Kontrolle. Dagegen legen besonders Angehörige, die die Selbständigkeit der Patienten wenig oder überhaupt nicht fördern, in vielen Situationen kontrollierendes Verhalten an den Tag. Im Gegensatz zu Frau Br. im obigen Beispiel, deren Kontrolle ihres Mannes auch als Aufforderung zur Selbstkontrolle interpretiert werden kann, da der Patient die Anrufe selbst bestimmt, übt Frau F. im folgenden Beispiel aktive Kontrolle über den Kopf ihres Mannes hinweg aus:

Frau F.: (...) Oder unterwegs beim Autofahren, er war ein leidenschaftlicher Autofahrer, ja, dann schimpft er, also da ist er dann // da kann ich ihn nicht beruhigen, und als er's letzte Mal hier war, da hat seine Hand, die linke Hand angefangen $\mathrm{zu}$ funktionieren, die geht ja nicht, und dann musste er ja jetzt aussetzen und da hat sich ja alles wieder zurückgebildet, und da ist er dann wirklich schon 'nen Meter mit dem Auto gefahren, und das fand er toll, ich hab den Schlüssel stecken lassen, geh rein zur Post, komm raus und denke: Mein Gott, das Auto ist ja weg, hab natürlich'n Schreck gekriegt, und, jetzt nehm ich'n Schlüssel mit, also da hab ich dann // also ich denke mir, dass er das packen würde, er ist ja nicht so, also, bloß es ist ja vom Körperlichen, man braucht ja beide Hände, also wir haben Automatik, und mit dem rechten Fuß das klappt schon, aber ich denke mal doch, und ich hab ihm das auch gesagt: Versprich mir, dass Du das nicht machst, Du wirst dann aggressiv, Du hast die Vorfahrt und Du möchtest die haben und das geht heute nicht mehr, die fahren ja wirklich, ich sag mal, wie die Henker, und wenn Sie zum Prenzlauer Berg fahren, also RÜCKSICHT -. (Z. 52-64)

Co-therapeutisches Engagement. Über rein pflegerische Tätigkeiten hinaus versuchen Angehörige, mit „co-therapeutischen“ Aktivitäten die Fähigkeiten der Patienten zu verbessern und damit langfristig die Selbständigkeit der Betroffenen zu erhöhen. So werden z. B. Übungen, die in der regulären Therapie durchgeführt werden, zu Hause weitergeübt, seien es krankengymnastische Aufgaben, Schreiben oder Rechnen, wie im folgenden Beispiel:

Frau M.: (...) Und Abziehen ging gar nicht - bin fast dran verzweifelt. Und nun hamwa beide, wenn wa schriftlich abgezogen haben, ooch unterschiedliche Methoden immer verwandt. Also, wenn ich jetzt von 7535 abziehe, des steht hier geschrieben, dann zähl ich 5 und 5 ist 10, 4 und 3 ist 7. Und mein Mann hat 
anders abgezogen. Jetzt konnte er mit meinem Schriftlichen gar nichts anfangen. Dann hab ich unsern Sohn gebeten: Du ziehst doch so ab wie Papa, mach doch det mal mit ihm. Und irgendwann platzte dann der Knoten. Ich hab dann diese Bleistiftergebnisse denn immer mit Bleistift hingeschrieben, ich hab die immer wieder wegradiert und immer wieder diesen Bogen benutzt und plötzlich ging des wieder, urplötzlich ging des wieder. Ich finde des heute noch'n Wunder. (Z. 528-537)

Stärkung des Kompetenzerlebens. Ähnlich wie die Bewältigungsstrategie „Zuspruch“ dient die Stärkung des Kompetenzerlebens dazu, die emotionale Situation des Patienten zu verbessern. Im Gegensatz zu der Strategie „Zuspruch“ soll die Absicht für den Pflegebedürftigen jedoch nicht offensichtlich sein. Durch die Einbeziehung des Patienten in Entscheidungsprozesse oder Alltagsprobleme soll verdeckt werden, dass die Erkrankung möglicherweise einen Kompetenzverlust mit sich gebracht hat.

Frau G.: (...) Aber wenn ich zur Bank war, dann kommt er schon und fragt jetzt und möcht 'nen Kontoauszug. Hat er früher nicht gemacht. Aber er möcht jetzt den Kontoauszug sehen, was drauf ist oder irgendwie. Aber ich mach regelrecht ... ich sag ihm alles, was ich ausgebe oder wofür ich einkaufe. Ich möchte schon seine Zustimmung haben, denn ich möchte ihn nicht so übergehen. Ich hab so das Gefühl oder ich möchte ihm das Gefühl geben, er ist noch immer der Wirtschaftsvorstand, so in dem Sinne, ja, er ist immer noch der Mann im Haus, auch wenn ich innerlich schon längst beschlossen hab oder versuche, das so zu machen. Aber ich muss ihn da irgendwie so hinbringen, die weibliche - wie sagt man? - Intuition oder irgendwie ... dieses Heimliche von hinten und so, und trotzdem ihn lenken und sagen: Du bist der Größte. Aber ich bin es doch, aber so in dem Sinne muss ich es machen, dass er sich bestätigt fühlt, er ist noch'n Mann, er ist nicht bloß'n kranker Mann. Das Selbstwertgefühl muss ich ihm irgendwie / (...). (Z. 418-428)

\section{Ichzentrierte Bewältigungsstrategien}

Sozialer Vergleich. Bei den ichzentrierten Bewältigungsstrategien konnte die Kategorie „sozialer Vergleich“ bei nahezu allen Angehörigen in der Stichprobe (10 von 11) gefunden werden. Ziel des Vergleichs mit anderen Betroffenen ist eine Stimmungsstabilisierung und Verbesserung der Motivationslage durch die Relativierung der eigenen Situation. So verhilft Frau G. der Vergleich mit anderen, Hoffnung zu schöpfen und weiterhin Geduld zu haben: 
Frau G.: (...) Und diese andern beiden Ehepaare, die haben wir im Urlaub kennen gelernt, und der Mann hat auch keine Sprache gehabt, sachtse, und er kann auch nicht laufen, er wird immer nur im Stuhl gefahrn, und da sag ich// vor acht Jahren// da sag ich: Aber ihr Mann sitzt doch und macht Kreuzworträtsel. Sagt sie: Fragen Sie nicht, was wir geübt, gemacht haben, und seiense froh, sie werden mit ihrem Mann auch wieder Kreuzworträtsel machen. Aber das dauert seine Zeit und nicht bloß Monate, sondern Jahre. Und daran halt ich mich so'n bisschen, ich sag mir: Jetzt ham wir zwei Jahre rum und die hat acht Jahre gebraucht. Also, ich hab dann noch'n bisschen Land. (LACHEN) (Z. 340-347)

Temporaler Vergleich. Ähnlich wie die Strategie „sozialer Vergleich“ zielen temporale Vergleiche ebenfalls auf eine Stimmungsstabilisierung und Verbesserung der Motivationslage ab. Das Bewusstmachen der Fortschritte des Patienten soll zu einer positiveren Bewertung der gegenwärtigen Situation führen. So machen sich Frau G. und Frau K. aus den folgenden Beispielen jeweils selbst Mut, indem sie sich vergegenwärtigen, dass sich die Situation im Vergleich $\mathrm{zu}$ früher schon erheblich verbessert hat:

Frau G.: (...) Na ja, aber das iss ... das ham wa ja alles nun schon halbwegs, die SCHLIMMSTEN ZEITEN, die haben wa, glaube ich, vorbei. (...)(Z. 109-110)

Frau K.: (...) Also, ich kann mit ihm'n Gespräch führen oder jemand anders kann mit ihm'n Gespräch führen. Und des war ja vorm dreiviertel Jahr überhaupt noch nicht möglich, ne. Da hat er ja nur gelegen und zugehört, aber nichts gesagt. Wenigstens spricht er schon, leiser'n bisschen und langsamer'n bisschen, aber es funktioniert und das ist gut. (...) (Z.96-100)

Akzeptieren. Die Strategie „Akzeptieren“ stellt ebenso eine häufig verwendete Form der Bewältigung dar (9 von 11 Angehörigen). Dass sie vom überwiegenden Teil der Angehörigen in der vorliegenden Stichprobe angewendet wird, ist sicherlich auch im Zusammenhang mit dem zeitlichen Abstand zum Schlaganfallereignis zu sehen: Bei den hier untersuchten Angehörigen waren zum Zeitpunkt der Interviews zwischen 14 und 74 Monaten seit dem Akutereignis vergangen, sodass bei vielen Angehörigen inzwischen eine Akzeptanz der bestehenden Einschränkungen eingetreten war.

Interviewerin: Gibt es im Vergleich zu früher doch Bereiche, die Sie durch die Pflege ihres Angehörigen vermissen oder eingeschränkt fühlen?

Frau K.: Na ja, fühlt man sich eingeschränkt, das iss ja ganz klar. Wie soll ich sagen, man kann zum Beispiel keine Ausflüge mehr machen. Ja, man kann jetzt 
nich sagen: Ich geh mal'n Eis essen. Oder Essen gehen, das geht eigentlich nicht, das iss ja nich möglich, im Moment ja nich. Vielleicht wird's mal wieder, aber im Moment nich. Das geht ja gar nicht, man kann ja nich sagen, so wie früher: Ach, wir fahren mal schnell nach Hamburg, einfach mal so. Das sind Sachen, die gehen einfach nich. Aber wie gesagt, es iss ... ist Vergangenheit. Es war schön, wir haben ein sehr schönes und aufregendes Leben gehabt. Na ja, nun iss es mal'n bisschen anders. Es iss jetzt nich so, dass ich mich unglücklich fühle dadurch oder dass ich des sehr vermisse. Es iss nicht mehr da, natürlich, ich nehm's zur Kenntnis, aber akzeptier ich so, wie's ist, nich. Ich meine, es geht nie im Leben so glatt, wie man sich das vorstellt. (LACHEN) Denk ich mal so, nich. (Z. 165-177)

Gutes im Negativen sehen. Auch die Strategie „Gutes im Negativen sehen“ ist für einen überwiegenden Teil der Angehörigen eine hilfreiche kognitive Bewältigungsstrategie. Die untenstehenden Zitate illustrieren, dass sich für manche Angehörige durch die Erkrankung sogar eine positive Veränderung in bestimmten Bereichen ergeben hat:

Frau K.: (...) Mein Mann hat sich positiv verändert, erstaunlicherweise, nich. Der ist nicht mehr so hektisch, nicht mehr so zappelig, nicht mehr so aggressiv. Also, das hat sich alles zum Besseren gedreht, nachdem er krank ist, des wär ja besser gewesen vorher, nich. (Z. 756-758)

Frau Be.: Also ES IST mitunter noch intensiver wieder geworden, ja, nicht. Also, möcht ich schon sagen, ja. (PAUSE) Ich kann eigentlich noch mehr mich in ihn reinfühlen (...). Es ist insofern intensiver geworden, dass wa sehr gut miteinander umgehen und wir uns aber beide auch besser anbrüllen können. Verstehense, wir können's besser rauslassen jetzt. (...) es hat sich durch die Krankheit meines Mannes doch vertieft, würd ich denken, bei beiden, doch. (Z. 381-390)

Einige Angehörige vermögen zwar keine partielle positive Veränderung im Vergleich zu früher zu sehen, sind sich aber dennoch positiver Aspekte ihrer Lage bewusst. So ist Frau F. dankbar, dass ihr Mann den Schlaganfall überlebt hat und kognitiv relativ unbeeinträchtigt ist:

Frau F.: (...) ich bin auf der anderen Seite auch wieder dankbar, dass er noch da ist, dass ich mich mit ihm unterhalten kann, wenn's auch nicht mehr so ist, wie's mal war, aber irgendwie, es sind doch immer wieder Phasen drin, wo man doch sagen kann, man ist glücklich wieder (...). (Z. 573-576) 
Distanzierung. Angst auslösende oder beunruhigende Gedanken können bei Angehörigen kognitive Mechanismen zur Folge haben, diese Überlegungen abzuwehren. Dies kann ein reflektierter Prozess sein, wie bei Frau G., oder aber auch unreflektiert erfolgen.

Frau G.: (SEUFZER) Wie gesagt, den Gedanken schieb ich immer recht weit weg, weil ich ihn mir nicht so vorstellen möchte: als Kind. Für mich isses ebent mein Mann. Deswegen erklär ich mir auch gar nich so richtig, wie weit des da oben kaputt ist oder nicht kaputt ist. Ich belüge mich manchmal im Stillen selber - aber nur so kommt man durch. (Z. 746-749)

Vergegenwärtigen der eigenen Erfolge. Diese Strategie hat eine Stärkung des Selbstwertgefühls und des eigenen Kompetenzerlebens zum Ziel, indem sich die Angehörigen die eigenen Erfolge im Umgang mit der Erkrankung und deren Folgen bewusst machen.

Frau K.: (...) jetzt muss ich DAS machen, was in meinen Kräften steht, nich. Ich muss alles machen, was ich kann, Papiere und Gewerbe abmelden und Auto und allet ... Verschiedenes machen. Also, es war schwierig, aber's hat funktioniert. (...) iss ungewohnt, so Rechnungen ausschreiben, oder äh, 'nen Scheck ausschreiben, hab ich nie gemacht in meinem Leben. Bin nie zur Bank gegangen - was soll ich'n da? Hab immer gesagt: Mach du das mal. Und er hat es ja immer gemacht. Ging ja bei ihm alles viel schneller, er war ja Geschäftsmann, was sollt ich da auch noch mitmachen, ja. Und so musste ich von einem Tag zum andern alles erledigen, ne. Dann musst ick erst mal hinjehn und fragen: Wie schreibt man denn eine Überweisung aus? Als Beispiel, es war wirklich für mich ... ich kam mir zwar manchmal blöd vor, aber es musste sein. Na ja, nun klappt des alles, jetzt geht's. (LACHEN) (Z.128-139)

Erholung. Diese Strategie umfasst alle Bemühungen, die einen Ausgleich zu den körperlichen und seelischen Belastungen der Pflege schaffen sollen. Diese können eine aktive oder passive Form annehmen. Im folgenden Beispiel wählt Frau A. eine aktive Form der Regeneration:

Frau A.: Ich setz mich manchmal auf mein Fahrrad, und fahre durch die Gegend, wo so schöne Luft ist; so'n bisschen kühl und so'n bisschen Wind. Und dann kommen schon die Gedanken - es klingt'n bisschen spinnig - dann denk ich: Hach, ist das Leben schön. Also, ich // wir sind ja gar keine anspruchsvollen Menschen gewesen, nie, wir lieben Natur und wir lieben so'n bisschen // tja, weiß ich was, eigentlich das einfache Leben, so nett mit dem Rad fahren, das ist z. B. für mich schon Hochgenuss. (Z. 315-320) 
Frau W. erholt sich dagegen eher in passiver Form:

Frau W.: Ich mach eben da und hier und irgendwann, wenn ich mal 'ne ruhige Minute hab, dann lass ich mich richtig fallen, ja, sitz so // sitz so auf der Couch, mach dann Fernsehen an oder Musik, meistens Fernsehen, guck aber gar nich hin oder ab und zu, hör gar nicht so zu, und bin dann richtig so in mich gekehrt, ja. Das entspannt, ganz locker, ja. (Z. 108-111)

Pflege der eigenen Bedürfnisse. Diese Strategie geht über die bloße Sorge für Erholung hinaus. Sie umfasst alle Anstrengungen der Angehörigen, neben der Pflegetätigkeit auch eigenen Interessen nachzugehen. Dies beinhaltet sich nicht nur über die Pflege zu definieren und sich gegebenenfalls auch gegenüber den Ansprüchen des Pflegebedürftigen hinwegzusetzen. Für Frau A. ist ihre ehrenamtliche Tätigkeit eine willkommene Möglichkeit, Abstand vom Pflegealltag zu gewinnen:

Frau A.: Ich habe eine ehrenamtliche Tätigkeit - Gott sei Dank - ich arbeite in einer durch Eigeninitiative im Diakoniezentrum gegründeten Bücherei mit, einmal in der Woche. Aber sobald auch noch Vertretungen anfallen, übernehme ich die mit Freuden. Es ist also mit meinem Fahrrad auch wieder bequem zu erreichen und da hab ich also immer an einem Nachmittag wieder zwei Stunden, nich. Und das mach ich mit Begeisterung, dort kommen Leute, da hab ich Gespräche und da kann ich auch mal völlig abschalten. Bücher liegen mir, also das ist nicht mein Beruf gewesen, aber ich mach das sehr gerne. (Z. 65-71)

Inanspruchnahme sozialer Unterstützung. Emotionale und instrumentelle Hilfeleistungen vom sozialen Umfeld $\mathrm{zu}$ erbitten fällt vielen Angehörigen schwer; angebotene Unterstützung wird dagegen meist angenommen. Emotionale Unterstützung wird vielfach von anderen Familienmitgliedern geleistet:

Frau Be.: (...) Mein Sohn ist jetzt 35 geworden, der ist nun über 1,80, dann sagt er immer: „Mami, komm mal, hat er dich wieder geärgert, der alte Vater? Stauch ihn zusammen, stauch ihn zusammen, halte durch Mami“, sagt er, „du bist auch wer.“ Also, so können wir miteinander umgehn und dann sagt er: „Also weeste Horst (gemeint ist sein Vater, d.h. der Patient), jetzt ist aber mal Schluss hier"(...). (Z. 432-436)

Aber auch der Angehörigenkurs oder die Gesprächsgruppe für Angehörige können eine wichtige Quelle für emotionale Unterstützung darstellen: 
Interviewerin: Was ist für Sie das Wichtigste, was Sie aus der Gesprächsgruppe mitnehmen?

Frau G.: (ÜBERLEGT) Irgendwie, dass man sich das Herz mal so richtig erleichtern kann, dass man mal alles so ablassen kann. Und vor allen Dingen, in einem Kreis ablassen kann, die VERSTÄNDNIS dafür haben und die nicht einfach dumme Fragen stellen oder dumme Ratschläge geben, die auf nichts basieren, weil sie keine Ahnung haben, wie geht man mit solchen Menschen um. (...) Und das gibt einem irgendwie dann doch wieder KRAFT, die nächsten vier Wochen durchzuhalten oder so. Weiter zu machen und nich sagen: Ach lass doch, ist doch gut. So nicht, selber wieder auch die Initiative bei uns aufputschen. (...) Doch ja, das // man wird doch wieder irgendwie so'n bisschen stabilisiert, wenn man rausgeht, irgendwie hier ... ich kann's nicht in Worte fassen, es ist irgendwie'n Gefühl: Du bist verstanden, wenn du deine kleinen Wehwechen sagst oder deine Problemchen, wenn du einmal nich weiterkommst und wie dir grade mal so ist. Und dann kannste auch mal alles loslassen und kannste auch alles mal sagen, auch wenn die Tränen dabei fließen. Aber man wird dann wieder getröstet und dann: Hach, war schön. Wie wenn ein Kind zur Mutter geht und sich eben trösten lässt. Das iss irgendwie ... das muss auch mal sein. (PAUSE) (...) (Z. 820-840)

Instrumentelle Unterstützung beinhaltete in der vorliegenden Stichprobe Fahrdienste, Reparaturen, Einkaufen gehen im Krankheitsfall und „Pflegevertretung“, damit Angehörige Arzttermine wahrnehmen oder den Kurs besuchen konnten, wie im folgenden Fall:

Frau M.: (...) Aber da muss ich sagen, hab ich mich nicht getraut, meinen Mann alleine zu lassen. Da war dann immer unser Sohn mit vonner Partie, dass er sich dann mit ihm beschäftigt hat. (Z. 306-308)

Inanspruchnahme professioneller Unterstützung. Knapp die Hälfte der hier untersuchten Angehörigen nehmen professionelle Unterstützung in Anspruch, um den Pflegealltag zu erleichtern. Die genutzten Dienstleistungen umfassten Mobilitätsdienst, Kurzzeitpflege, Haushaltshilfe sowie Pflegedienst.

Frau M.: Zuerst hatt ich auch noch - nach der Lungenentzündung - morgens immer 'ne Hauskrankenpflege und diese Frau, die da kam, die hat mir des denn auch alles noch besser zeigen können, als wenn ich mich da selber versucht hätte. Sie hatte die ersten Male meinen Mann gebadet. Sie hatte dann zwar immer nur einmal pro Woche dafür Zeit, aber det war wunderbar, diese Hilfestellung. Und dann hab ich's zwischendurch noch an einem anderen Wochentag probiert. Und nachdem die nun nicht mehr kommen konnte, von der Kasse kriegt ick des nicht mehr, des hab ich nur bis Ende Dezember gekriegt. 
Dann hat ich mir des noch zwei Monate selbst bezahlt, Januar und Februar noch, und dann hamwa des auslaufen lassen. (Z. 209-217)

Emotionsausdruck. Einige Angehörige verschaffen sich durch Weinen oder lautes Schimpfen die nötige Erleichterung in emotional belastenden Situationen. Zum überwiegenden Teil verbergen die Angehörigen solche Ausbrüche vor den Pflegebedürftigen, in Einzelfällen kommt es jedoch auch in Anwesenheit der Patienten zu solchen Vorfällen. Bei Frau So. trifft beides zu:

Frau So.: Und da wird er dann, mein kleiner Mann, öfter mal ein Choleriker außerdem noch, ja. Dann wirds 'n bisschen laut bei uns, ja. Kann auch sein, dass die Nerven nu inzwischen bei mir auch ein bisschen angeschlagen sind, weil die Einsicht bei ihm überhaupt nicht vorhanden ist, wenn ich ihm dann sage: Ich kann das jetzt nicht machen, ich muss das noch machen und dies noch machen und so. Dann hält er mir vor, ich soll ihm nicht immer alles erzählen, was ich noch mache, wie fleißig ich bin und ja: Ja, du bist Bestarbeiter. Und so weiter. Ja, solche Sachen kommen dann. Dann wird er auch recht ungezogen.

Interviewerin: Und wie ist das für Sie?

Frau So: Na, da geh ick raus inne Küche, setz mich hin und fange an zu heulen, so wie jetzt ooch beinah (TRÄNENERSTICKTE STIMME), ja. Und das ist mehrmals in der Woche. Ich meine, man verlangt ja nicht, dass er nu für jede Handreichung, die man macht, danke sagt, aber bisschen Anerkennung möchte man auch ganz gerne haben, ne. (Z. 75-86)

Substanzgebrauch. Immerhin für knapp die Hälfte der hier befragten Angehörigen (5 von 11) war Substanzgebrauch in Form von Alkoholgenuss oder Einnahme von Beruhigungsmitteln oder Schlafmitteln eine probate Strategie, um eine kurzzeitige emotionale Entlastung herbeizuführen. Bemerkenswert war die recht große Offenheit mit der die Interviewpartnerinnen ihre Strategien sich selbst zu betäuben schilderten:

Frau A.: Also, mal jetzt ganz ehrlich zu Ihnen gesagt: Es gibt auch Situationen, wo ich mal 'ne Flasche Wein hochhole und denke: So, jetzt begieß ich mal meine Nase. Ich meine, ich besauf mich nich, dass ich unter den Tisch falle (LACHEN) oder zu torkeln anfange, sondern ich trink nur so meistens zwei Viertel. Es iss ja nich so furchtbar viel, aber'n halber Liter iss es halt und das hält mich auch'n bisschen weg. Aber das mach ich nur abends. Also, ich hab was gegen Betrunkene, aus Erfahrung mit meinem Mann von früher. Ich hab was gegen betrunken sein oder Leute, die sich nich im Griff haben, und deshalb würd ich nie BEI TAGE sowas machen, aber abends, wenn ich mich dann ins Bett legen kann: So, jetzt schlaf ich gut - aber selten, nicht immer öfter, nicht zur Gewohnheit. Aber es gibt ja auch so Schwächen. (Z. 389-398) 
Frau Be.: (...) Dann hab ich meine Schlaftablette genommen und weg war ich. Aber dann war ich ooch betäubt, völlig betäubt und NUR SO ging es dann. (...) (Z. 250-251)

Religiöse Bewältigung. Religiöse Überzeugungen können eine sinnstiftende Funktion in der Bewältigung der Pflegesituation einnehmen. Dass in der vorliegenden Stichprobe die Strategie „religiöse Bewältigung“ nicht häufiger zu Tage trat (lediglich bei 2 von 11 Angehörigen), hängt vermutlich mit regionalen Gegebenheiten zusammen. In Regionen mit traditionelleren Lebensformen als in einer säkularisierten Metropole wie Berlin wäre sicherlich eine häufigere Verwendung dieser Bewältigungsstrategie zu erwarten. Außerdem stammten 5 der 11 Interviewpartnerinnen aus dem ehemaligen Ost-Berlin.

Interviewerin: Gibt es in der derzeitigen Situation etwas, was Ihnen Trost gibt oder Ihnen hilft?

Frau M.: (ÜBERLEGT) Na ja, sagen wa mal so: Man hat ja vielleicht immer mal wieder den Hang anzunehmen, dass es'n lieben Gott gibt, ja. Und des iss natürlich auch'n Umstand, der einem weiterhilft, ja. (PAUSE) Ich bin kein fleißiger Kirchgänger, so ist des nicht, aber ick bin eigentlich so erzogen worden, dass ick mich immer wieder damit beschäftigt habe, und irgendwie ... doch, das hat mir geholfen. (Z. 650-654)

\subsection{Gesamtbeurteilung des Bewältigungsverhaltens}

Auf der Grundlage der detaillierten Analyse des Bewältigungsverhaltens anhand des oben dargestellten Kategoriensystems erfolgte eine Gesamtbeurteilung des Bewältigungsverhaltens für jede der befragten pflegenden Angehörigen durch zwei Rater (die Autorin und ein externer Intervisor, siehe Kapitel 7.5). Dabei sollten folgende Aspekte bei der Gesamtbeurteilung der Bewältigung berücksichtigt werden: Die Menge der verwendeten Bewältigungsstrategien, das Ausmaß der praktischen/pflegerischen Unterstützung des Pflegebedürftigen; das Vermögen der Angehörigen, eigenen Interessen nachzugehen; die Fixierung auf den Pflegebedürftigen; das Ausmaß der sozialen Unterstützung; sowie die Gesamtbelastung durch die Pflegesituation. Diese Aspekte dienten als Orientierungshilfe, um zu einer besseren Gesamteinschätzung der Bewältigung zu gelangen. Die Einschätzung der Bewältigung erfolgte anhand einer fünfstufigen Skala mit den Endpunkten 1 (funktional) und 5 (dysfunktional). Tabelle 14 zeigt die Ergebnisse der Gesamteinschätzungen durch die Rater mit dem aus den 
Beurteilungen gebildeten Mittelwert. Zur Bestimmung der Interrater-Reliabilität wurde die Rangkorrelation (Spearman's rho) berechnet, die mit $r_{\rho}=0.9$ sehr gut ausfiel.

Tabelle 14: Gesamtbeurteilung des Bewältigungsverhaltens der pflegenden Angehörigen

\begin{tabular}{cccccc}
\hline Nr. & Frau & Gruppe & $\begin{array}{c}\text { Gesamtbeurteilung } \\
\text { Bewältigung } \\
\text { Rater 1 }\end{array}$ & $\begin{array}{c}\text { Gesamtbeurteilung } \\
\text { Bewältigung } \\
\text { Rater 2 }\end{array}$ & $\begin{array}{c}\text { Mittelwert der } \\
\text { Gesamtbeurteilung }\end{array}$ \\
\hline 1 & G. & T & 2 & 1 & 1.5 \\
2 & W. & T & 4 & 4 & 4 \\
3 & A. & T & 2 & 2 & 2 \\
4 & K. & T & 3 & 2 & 2.5 \\
5 & T. & T & 5 & 5 & 5 \\
6 & M. & T & 1 & 1 & 1 \\
7 & So. & K & 5 & 5 & 5 \\
8 & F. & K & 5 & 5 & 2.5 \\
9 & Be. & K & 2 & 3 & 3 \\
10 & Sn. & K & 3 & 3 & 2 \\
11 & Br. & K & 2 & 2 & 5 \\
\hline
\end{tabular}

Anmerkungen: $\mathrm{T}=$ Treatmentgruppe, $\mathrm{K}=$ Kontrollgruppe. Die Einschätzung der Bewältigung erfolgte anhand einer fünfstufigen Skala mit den Endpunkten 1 (funktional) und 5 (dysfunktional).

\subsection{Vertiefende Fallanalysen}

Im dritten Schritt der Auswertung der qualitativen Daten wurden vertiefende Fallanalysen erstellt. Für die vertiefenden Fallanalysen wurde zunächst die durch die Rater vorgenommene Gesamtbeurteilung des Bewältigungsverhaltens der Angehörigen in Beziehung gesetzt zu den Ergebnissen aus dem quantitativen Teil der Untersuchung. Tabelle 15 liefert dazu die Übersicht. 
Tabelle 15: Belastungswerte der pflegenden Angehörigen zu den Messzeitpunkten 1 und 4 und Gesamtbeurteilung des Bewältigungsverhaltens

\begin{tabular}{|c|c|c|c|c|c|c|c|c|c|}
\hline Nr. & Frau & Gruppe & $\begin{array}{c}\text { ADS } \\
\text { (MZP 1) }\end{array}$ & $\begin{array}{c}\text { BL } \\
\text { (MZP 1) }\end{array}$ & $\begin{array}{c}\text { SAS } \\
\text { (MZP 1) }\end{array}$ & $\begin{array}{c}\text { ADS } \\
\text { (MZP 4) }\end{array}$ & $\begin{array}{c}\text { BL } \\
\text { (MZP 4) }\end{array}$ & $\begin{array}{c}\text { SAS } \\
\text { (MZP 4) }\end{array}$ & $\begin{array}{c}\text { Mittelwert } \\
\text { Gesamt- } \\
\text { beurteilung }\end{array}$ \\
\hline 1 & G. & $\mathrm{T}$ & 8 & 14 & 32 & 16 & 15 & 31 & 1.5 \\
\hline 2 & W. & $\mathrm{T}$ & 15 & 40 & 41 & 25 & 41 & 43 & 4 \\
\hline 3 & A. & $\mathrm{T}$ & 7 & 7 & 25 & 7 & 12 & 25 & 2 \\
\hline 4 & $\mathrm{~K}$. & $\mathrm{T}$ & 32 & 18 & 32 & 12 & 10 & 25 & 2.5 \\
\hline 5 & T. & $\mathrm{T}$ & 24 & 48 & 54 & 32 & 49 & 45 & 5 \\
\hline 6 & M. & $\mathrm{T}$ & 17 & 28 & 43 & 15 & 20 & 32 & 1 \\
\hline 7 & So. & K & 13 & 22 & 30 & 27 & 37 & 50 & 5 \\
\hline 8 & F. & $\mathrm{K}$ & 23 & 47 & 47 & 30 & 60 & 54 & 5 \\
\hline 9 & Be. & K & 29 & 44 & 52 & 19 & 38 & 43 & 2.5 \\
\hline 10 & Sn. & K & 10 & 34 & 25 & 14 & 27 & 31 & 3 \\
\hline 11 & Br. & K & 9 & 8 & 23 & 0 & 0 & 20 & 2 \\
\hline
\end{tabular}

Anmerkungen: $\mathrm{T}=$ Treatmentgruppe, $\mathrm{K}=$ Kontrollgruppe; MZP 1: Messzeitpunkt 1; MZP 4: Messzeitpunkt 4; ADS: Allgemeine Depressionsskala; SAS: Self-Rating Anxiety Scale; B-L: BeschwerdenListe; fettgedruckte Werte kennzeichnen solche, die oberhalb des Cutoffs für die entsprechende Variable liegen und deuten auf Auffälligkeiten im jeweiligen Bereich hin. Die Grenzwerte liegen bei: $\operatorname{ADS} \geq 23$; $\mathrm{SAS} \geq 36$; B-L $\geq 25$.

Da im Rahmen dieser Arbeit in erster Linie interessierte, inwiefern Interaktionen zwischen dem Bewältigungsstil und der Effektivität der Intervention vorliegen, wurden zwei Teilnehmerinnen am Angehörigenkurs ausgewählt, die in der Gesamtbeurteilung den höchsten und niedrigsten Wert in Bezug auf funktionales Bewältigungsverhalten erreichten. Sie unterscheiden sich nicht nur im Bewältigungsverhalten, sondern auch in der Entwicklung der Belastungswerte über den zeitlichen Verlauf. Es wird zunächst Frau T. vorgestellt. Zum Messzeitpunkt 1 wies sie hohe Belastungswerte auf, ihr Bewältigungsverhalten wurde von den Ratern insgesamt als patientenfixiert und dysfunktional eingeschätzt. Die Belastungswerte liegen im zeitlichen Verlauf durchgängig oberhalb des Grenzwertes im pathologischen Bereich, allerdings nimmt die Angst im Vergleich zu den anderen erhobenen Variablen kontinuierlich ab. Zwar ist das Bewältigungsverhalten insgesamt als patientenfixiert zu bewerten, es sind jedoch erste 
positive Veränderungen zu verzeichnen. Ihr gegenübergestellt wird Frau M., die ebenso wie Frau T. zum Messzeitpunkt 1 hohe Belastungswerte aufwies, jedoch ein insgesamt funktionales Bewältigungsverhalten zeigte. Im zeitlichen Verlauf nehmen alle Belastungswerte ab. Nach einer ausführlichen Analyse des Bewältigungsverhaltens der beiden Angehörigen wird diskutiert, inwiefern das Bewältigungsverhalten Einfluss auf die Teilnahme an der Intervention und auf die Entwicklung der Belastungswerte hatte.

\subsubsection{Frau T.: „Man will doch gar nichts Besonderes, man will ja nur mal eben frei sein."}

Das Interview mit Frau T. fand im September 1998 statt. Zu diesem Zeitpunkt war sie 58 Jahre alt, ihr Mann war 77 Jahre alt. Im April 1994 erlitt Herr T. einen linksseitigen zerebralen Insult mit armbetonter Hemiparese rechts. Neuropsychologisch waren bei Aufnahme in der Tagesklinik keine Defizite zu verzeichnen, allerdings litt er an einer Depression, die sich während des Aufenthaltes in der Klinik jedoch zurückbildete. An Risikofaktoren wies der Patient eine koronare Herzkrankheit, einen arteriellen Hypertonus sowie eine Hypercholesterinämie auf. Außerdem lag eine Kriegsverletzung der linken Hüfte vor. Der Schlaganfall trat während eines Urlaubs auf Mallorca ein, kurz nachdem Frau T. in Rente gegangen war. Sie hatte als Kontoristin gearbeitet. Ihr Mann arbeitete bis 1985 als Feinmechaniker und war mit 63 Jahren in Rente gegangen. Das Paar hat 11 Jahre lang unverheiratet zusammengelebt, war zum Zeitpunkt des Interviews seit 17 Jahren verheiratet und hatte keine Kinder. Sie lebten in einem Berliner Innenstadtbezirk in einer 3-Zimmer Wohnung.

Aus Tabelle 16 ist zu entnehmen, dass bei Frau T. zu allen Messzeitpunkten ein deutlich erhöhtes Ausmaß an Depression, Angstsymptomen und körperlichen Beschwerden vorlag. Während die Angstsymptome im zeitlichen Verlauf im Vergleich zum Ausgangsniveau abnahmen (jedoch immer noch pathologisch erhöht waren), stieg das Ausmaß der depressiven Symptome an. Die körperlichen Beschwerden bewegten sich $\mathrm{zu}$ allen Messzeitpunkten auf einem gleichbleibend hohen Niveau. 
Tabelle 16: Ausprägung von Depression, Angst und körperlichen Beschwerden zu den 4 Messzeitpunkten bei Frau T.

\begin{tabular}{lcccc}
\hline Variable & $\begin{array}{c}\text { Messzeit- } \\
\text { punkt 1 }\end{array}$ & $\begin{array}{c}\text { Messzeit- } \\
\text { punkt 2 } \\
(+5 \text { Monate })\end{array}$ & $\begin{array}{c}\text { Messzeit- } \\
\text { punkt 3 } \\
(+10 \text { Monate })\end{array}$ & $\begin{array}{c}\text { Messzeit- } \\
\text { punkt 4 } \\
(+16 \text { Monate })\end{array}$ \\
\hline Depression (ADS) & & & & \\
Angst (SAS) & 24 & 26 & 36 & 32 \\
körperliche Beschwerden (B-L) & 48 & 46 & 49 & 45 \\
& & & & 49 \\
\end{tabular}

Anmerkungen: ADS: Allgemeine Depressionsskala; SAS: Self-Rating Anxiety Scale; B-L: Beschwerden-Liste. Fettgedruckte Werte kennzeichnen solche, die oberhalb des Cutoffs für die entsprechende Variable liegen und deuten auf Auffälligkeiten im jeweiligen Bereich hin. Die Grenzwerte liegen bei: ADS: $\geq 23$; SAS: $\geq 36$; B-L: $\geq 25$.

\section{Pflegerische Aufgaben}

Die pflegerischen Tätigkeiten von Frau T. umfassen Unterstützung bei der Körperpflege, bei Toilettengängen und beim Anziehen. Bei längeren Wegstrecken muss sie ihren Mann im Rollstuhl schieben. Der Barthelindex (siehe Kap. 4.3.4 Unterpunkt „Ausmaß der Pflegebedürftigkeit“) liegt bei allen 4 Messzeitpunkten bei 35 Punkten. Den Haushalt führt sie ohne weitere Unterstützung. Sie schildert ihren Ehemann als sehr bequem, der Tagesablauf richte sich komplett nach seinen Bedürfnissen, er bestehe darauf, dass die Zeiten für diverse Aktivitäten eingehalten werden. Den Zeitaufwand für die Pflege beziffert sie zum Messzeitpunkt 1 mit 6 Stunden, zum Messzeitpunkt 4 (16 Monate später) mit 4 Stunden. 12 Stunden am Tag müsse sie für ihren Angehörigen erreichbar, also anwesend sein. Ihr Mann ist in die Pflegestufe 1 eingestuft worden. Einen Einblick in ihren normalen Tagesablauf geben folgende Ausschnitte:

Frau T.: Ja, na erst mal fängt das so an, dass ich sehr gebunden bin. Ich würde selbst mein Tag ganz anders gestalten. (VERHALTENES LACHEN) Gezwungenermaßen ist es so, dass wir um sieben aufstehn, das heißt: ich, kurz nach den Nachrichten. Dann wasch ich mich, dann geh ich in die Küche und mach Kaffee. Mittlerweile geht mein Mann ins Bad, dann versucht er // die Zähne macht er sich und soweit er alleine kommt und wenn's denn soweit ist, komm ich, mach ihm unter de Arme oder, ja. Und dann helf ich ihm soweit, bis 
er fertig ist, und dann trinken wa beide Kaffee. Dabei geh ich schon mal runter, hol 'ne Zeitung, dann zieht er sich noch mal den Schlafanzug an und liegt mit der Zeitung ins Bett. (Z. 4-11)

Frau T.: (...) wenn er fertig ist, so um zehn, dann mach ich ihm Augentropfen und um halb elf komm ich dann rein und sage: Jetzt musste aber uffstehn. Denn er kann ja nich so lange liegen. Na, und denn zieh ich ihn an. Jo und denn, was machta denn eigentlich? Meistens war ich vorher schon unten, von neun bis zehn, hab schon das Nötigste eingeholt, jo. Was machta denn? Setzt er sich vorne hin, guckt inne Illustrierte, hört wieder Radio und legt sich meist wieder lang auf de Couch, was mir ja eigentlich gar nicht so gut gefällt. Aber ich krieg ihn nicht dazu rauszukommen. (Z. 23-29)

Insgesamt scheint Frau T. vor seiner Bequemlichkeit kapituliert zu haben wie der folgende Interviewausschnitt verdeutlicht:

Interviewerin: Sie sagten auch vorhin (...), dass er so'n bisschen verwöhnt ist ... Frau T.: Ick hab ihn zum Anfang zu sehr verwöhnt, ick hab mir gedacht // fing schon an, bloß wenn er die inner Hand hielt: Brille muss geputzt werden. Jetzt kommt er schon an in der Küche, wenn ich noch beim Mittagkochen bin, damit er zum Mittag für seinen Fernseher die Brille geputzt hat, aber auch jeden Tag um die gleiche Zeit. Det nervt allet so, ick mein, det macht man ja im Vorbeigehen, aber ... des iss furchtbar. (...) Was soll man machen? Ich weiß et nich mehr, ich muss so weiter machen. (Z. 192-206)

Aus dem letzten Satz spricht Resignation, sie hat sich in die Rolle gefügt, ihrem Mann vieles abzunehmen. Bemerkenswerterweise gibt es jedoch einen Bereich, in dem sie die Selbständigkeit ihres Mannes fördert: Die nächtlichen Toilettengänge ihres Mannes begleitet sie nicht.

Frau T.: (...) alle zwei Stunden muss er auf Toilette pullern, aber das macht er jetzt alleine, det hab ich jetzt nich mehr jehört. Det nervt ja auch, wenn man jede Nacht raus muss. $\mathrm{Na}$ ja, 'n bisschen besser isses doch geworden gegen früher. Man kriegt det ja nich immer so mit, aber da war's noch schlimmer.

(Z. 373-376)

\section{Verwendete Bewältigungsstrategien}

Insgesamt verfügt Frau T. über relativ wenig Bewältigungsstrategien. Ihre Erzählung lässt die Verwendung von drei patientenzentrierten Bewältigungsstrategien erkennen, die primär eine Verbesserung des Zustandes des Patienten bewirken sollen, gegenüber fünf ichzentrierten Bewältigungsstrategien. Eine Analyse der patientenzentrierten 
Bewältigungsstrategien ergibt folgendes Bild: Wie bereits aus den obigen Ausschnitten deutlich wird, bemüht sie sich kaum, die Selbständigkeit ihres Mannes zu fördern. Der Schonraum, den sie ihrem Mann zugesteht, zeigt sich auch in ihrem Bemühen, jegliche psychische Belastungen von ihm fern zu halten (Fernhalten psychischer Belastungen). So nahm Frau T. ihren Mann zur Kur mit, weil keine der Kurzzeitpflegeeinrichtungen, die sie sich angeschaut hatte, ihren Ansprüchen genügte. Dass die Kur ihr dann nicht den gewünschten Effekt brachte, dürfte nicht verwundern:

Frau T.: (...) Wir waren jetzt zur Kur und das war leider auch nicht so, wie ich mir das dachte. (...) Ich mein, wenn einer alleine ist und behindert, ist det ganz toll. Aber für mich war det nu überhaupt nich. Det warn ja nur echt Kranke, wollte ja auch nach vier Jahren endlich mal Urlaub haben. Und ich hatte eigentlich für mich 'ne Kur alleine eingereicht. Und die wurde ooch genehmigt und nu ging's darum // bin ich rumgerannt, wo lass ich meinen Mann. Hab ich gesagt: Du musst drei Wochen inne Kurzzeitpflege. Ach, ick war überall, wie ich det gesehen hab, wie die Leute da sitzen, die sitzen echt nur und warten auf Essen oder um die Zeit tot zu kriegen. Also, mir hat keins gefallen und denn hab ich mir gesagt: Nach Hause kommen kann ooch nicht jeden Tag jemand. Meine Schwester, die wohnt in Hermsdorf, det geht ooch nicht. Dann hat er gesagt: Na, denn komm ich mit, du hast doch da so'n schönes Prospekt, da nach Bevensen. (Z. 93-104)

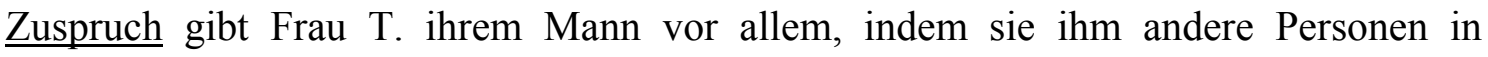
ähnlicher Lage vorhält, die im Vergleich zu ihrem Mann jedoch einen stärkeren Willen an den Tag legen ihr Rehabilitationspotential auszuschöpfen. Dies kumuliert in der Bemerkung:

Frau T.: (...) ich sag, ,wenn man muss, dann geht alles, man muss nur wollen“. (Z. 388)

Die Strategie Informationssuche ist ein wichtiges Motiv für die Teilnahme am Angehörigenkurs, wobei dieses eher zweitrangig erscheint neben dem Beweggrund, durch den Austausch mit anderen eine emotionale Entlastung $\mathrm{zu}$ erfahren. Dies illustriert der folgende Ausschnitt:

Frau T.: (...) Und det fand ich ganz gut, det es so viel Leute gibt ... und det gibt ja welche, denen's viel schlimmer geht, der Mann im Krankenhaus iss und schon ewig. Wie das alles so weitergeht mit den Behörden ... ochhh, det gibt ja so viel schlimme Sachen, det iss ja furchtbar. Interviewerin: Und was war so Ihre Motivation, am Kurs teilzunehmen?

Frau T.: Ja, ich hab gedacht: Na ja, man hört ja immer des, was es ooch so gibt, 
was ich nicht wusste, mit Freistellungsuffträje oder mit dem Fahrdienst und mit dem Sozial...// (...)

Interviewerin: Also es war dann schon so, dass Sie sich auch erhofft haben ...

Frau T.: Ja, wenn ... man hat ja doch immer wie gesagt ... es hilft einem ja auch immer, wenn's andern schlechter geht. Ich mein, so schlecht geht's mir doch gar nicht. (...) (Z. 549-564)

Neben der Komponente, durch Sammeln von Informationen die Situation ihres Mannes zu verbessern, erfüllt die Strategie Informationssuche bei Frau T. also auch die Funktion einer ichzentrierten Bewältigungsstrategie, indem soziale Vergleiche ermöglicht werden. Durch die Beobachtung, dass andere in einer noch schlimmeren Lage sind (ihrer subjektiven Wahrnehmung nach), verschafft sich Frau T. immer wieder die Motivation, weiter durchzuhalten.

Interviewerin: Sie haben sich ja dazu entschlossen, die Gesprächsgruppe weiterzumachen nach dem Kurs. Und was warn da die Gründe für Sie, da noch weiterzumachen?

Frau T.: Mal unter Leute zu sein denen's genauso geht wie mir. Det is schon mal schön. Und wie gesagt, denen geht's viel schlechter und da bin ich immer froh, dass mir da noch gut geht. Manche haben auch vielleicht finanzielle Sorgen oder so ... wie die damit umgehen, is immer interessant zu hören. Des is, gloobe ich, ooch noch schlimm, wenn jemand jetzt noch det Geld fehlt, das würd ooch nochmal anders sein, würd ich sagen. (Z. 589-595)

Im folgenden Gesprächsausschnitt berichtet Frau T. von einer Situation, in der ihr Mann ihr anhand eines sozialen Vergleiches vorhält, dass es ihr relativ gesehen gut gehe. Gemessen an äußeren Aspekten stimmt sie ihrem Mann zu, im letzten Satz wird jedoch eine gewisse Ambivalenz deutlich, dass sie dies in emotionaler Hinsicht nicht so empfindet:

Frau T.: Ja, wenn ... man hat ja doch immer wie gesagt ... es hilft einem ja auch immer, wenn's andern schlechter geht. Ich mein, so schlecht geht's mir doch gar nicht. Mein Mann sagt immer: Dir geht's doch gut, wat hast du denn. Ich weiß gar nich, wat du hast. Dir geht's gut, du hast doch alles, was zu essen, zu trinken, Geld haste genug, wat willste denn noch.

Interviewerin: Und was denken Sie dann?

Frau T.: Mensch, wenn du wüsstest: Meine Ruhe will ich haben. (PAUSE) (Z. 464-469)

Der letzte Satz kann doppeldeutig verstanden werden, es spricht daraus nicht nur das Bedürfnis nach mehr Ruhe und Erholung im Pflegealltag, sondern auch der insgeheime Wunsch nach einer Beendigung der gesamten Pflegesituation. Davon, tatsächlich die 
Pflege ihres Mannes ganz aufzugeben, ist Frau T. jedoch weit entfernt. Und auch für mehr Erholung und Entspannung für sich zu sorgen, gelingt Frau T. kaum. In den Zeiten, in denen ihr Mann sich auf dem Sofa ausruht, was mehrfach am Tag geschieht, gönnt sich Frau T. keinesfalls eine Ruhepause (Erholung):

Interviewerin: Und was machen Sie so in der Zeit (Anmerkung: in der der Mann auf dem Sofa liegt)?

Frau T.: Na ja, ich mach meist sauber. Man hat ja immer was zu tun, nur so zu Hause rumbruddeln, befriedigt mich natürlich auch nich. Ich würde gerne mal weggehen oder so. Aber das gefällt ihm ja auch nicht, der meint immer, ick hätte nur mein Vergnügen im Kopf. Wenn ich Einkaufen gehe, ist det schon Vergnügen. Is ja wirklich kein Vergnügen heutzutage, nich. Ja, mein Mann is auch sehr egoistisch, der meint, es ist eben so und ich müsste mich ebent danach richten. (...) (Z. 50-57)

Dabei gäbe es durchaus Ansatzpunkte für erholsame Unternehmungen, die auch ihr Mann billigen würde:

Interviewerin: Ja, tun Sie denn sonst im Moment etwas für sich?

Frau T.: Nee, nee ... da is gar nichts. Müsste mich mal wieder mit meine Kolleginnen treffen, aber jetzt nach'm Urlaub hatten wa so viel anderes, ne. Sonst eigentlich nichts. Müsste mal Schwimmen gehn ... müsste, müsste, müsste. Man sacht immer „müsste“, aber du machst nichts. Denn det würd er noch einsehn, wenn ich Schwimmen gehe, des hat er nämlich früher auch immer gemacht. (...) (Z. 234-239)

Allenfalls fährt sie gelegentlich mit dem Auto für zwei Stunden durch die Stadt, wenn sie Abstand braucht, was allerdings im Berliner Autoverkehr nicht gerade als entspannende Tätigkeit bezeichnet werden kann:

Frau T.: (...) Wie gesagt, manchmal, wenn's mir zuviel ist, dann nehm ich meine Tasche und haue mit dem Auto zwei Stunden ab: Musste mal sehn, was hier die Baustelle (Anmerkung: am Potsdamer Platz) macht. Und dann bin ich mal so 'ne Runde alleine gefahrn, und det war's denn. (PAUSE, SEUFZER) (Z. 454-455)

Wie sehr sie selber durch ihr Verhalten dazu beiträgt, dass ihr Leben so stark durch die Bedürfnisse ihres Mannes diktiert wird, zeigt folgendes Zitat: 
Interviewerin: Sie sagten am Anfang, Sie hätten sich den Tagesablauf ganz anders vorgestellt oder Sie würden sich den ganz anders gestalten.

Frau T.: Na ja, früher. Wir wollten ja jetzt eigentlich was unternehmen jetzt, wo wa Rentner sind. Wollten wa verreisen und eben alles, Umland fahren, Museen und sowas, was man ja, wenn man arbeitet, keine Zeit hat, ja. Das wollten wa nun machen und nu is det alles plötzlich vorbei. Und alleine, det lohnt immer nich. Ich muss zum Beispiel, wenn ich um eins losgehe, muss ich um drei wieder da sein, muss ich ihm Kaffee machen. Das ist dann zu kurz die Zeit. Mal macht er sich auch Kaffee, kann er schon alleine kochen. Obwohl Kaffee geht dann daneben, aber is ja nich so schlimm.

Interviewerin: Das kann er auch alleine.

Frau T.: Das kann er. Kaffee könnt er sich kochen, wenn ich ihm dann Kuchen hinstelle. Hab ich früher auch gemacht, hab mich mit meiner Kollegin getroffen, dann warn wa im Grunewald mal spazieren, 'n paar Stunden. Aber so, dass ich um fünf immer wieder da bin, ja, zum Abendbrot eben. Er is auch nicht gern alleine, er muss immer wissen, was ich mach. Na ja, was mach ich? Man will ja gar nichts machen, man möcht nur'n BISSCHEN FREIER sein, ja ... des Gebundene, des macht einen so krank. Und er will auch keine fremden Leute haben. (...) (Z. 77-92)

Erstaunlicherweise hat Frau T. es dennoch nicht völlig aufgegeben, für etwas mehr Freiraum zu kämpfen. So will sie gerne zu einem Familientreffen fahren, was ihr Mann jedoch nicht billigt. Nachdem sie schon von ihrer Ärztin Zuspruch bekommen hat, will sich Frau T. auch von der Interviewerin Bestätigung einholen, wie das folgende Zitat zeigt:

Frau T.: (...) Nun weiß ick noch nicht, ob ich det mache oder nich. Ich steh immer noch zwischen Baum und Borke. Mit der Ärztin hatt ich schon mal gesprochen, die bei uns zu Hause kommt, und die sagte // weil ich sach, ich möchte gerne mal raus, Urlaub machen, und wenn's nur 14 Tage sind. „Ja“, sagtse, „müssense mal anfangen. Ich würd nich gleich vier Wochen fahren, aber wennse mal zwei, drei Tage fahrn. Und fangense mit ein Tag an." $\mathrm{Na}$, nu isset so, dass wir drei Tage weg wollen, Freitag, Samstag, Sonntag. Die (Anmerkung: die Familie ihrer Schwester) wollen mich mitnehmen.

Interviewerin: Also eigentlich zwei volle Tage.

Frau T.: Ja, genau. Ruhe hab ick natürlich nicht. Aber ick will einfach mal probieren. Was meinen Sie? Ob ich det kann? Meinense?

Interviewerin: Ja.

Frau T.: Schlechtes Gewissen hab ich trotzdem, weil er sagt: Du bist egoistisch und denkst nur an Vergnügen. Sag ich: Ich will mich doch nicht vergnügen, ick will nur mal hier raus, weiter will ich doch gar nichts.

Interviewerin: Was denken Sie denn, wenn er so sagt: Sie sind egoistisch?

Frau T.: Umgekehrt ist det eigentlich, wissense. Er ist nämlich ... der gönnt eem keene Freude - nüscht. Das is furchtbar. (Z. 153-169) 
Auch in der Gesprächsgruppe eine Woche nach dem Interview erwähnt sie ihren Wunsch, zum Familientreffen zu fahren. Die anderen Frauen sprechen ihr Mut zu, sodass sie sich tatsächlich schließlich gegenüber ihrem Mann durchsetzt und die Fahrt organisiert. Da ihr Mann unter keinen Umständen in eine Kurzzeitpflegeeinrichtung will, bittet sie die Nachbarin, zweimal am Tag nach ihrem Mann zu sehen, was angesichts der folgenden Aussage ein großer Schritt für Frau T. ist (Inanspruchnahme sozialer Unterstützung):

Interviewerin: Aber würden Sie denn noch Leute kennen, die Sie um Hilfe bitten könnten - eigentlich?

Frau T.: Ich bin selber so, ich mag immer keinen belästigen, wissense. Ich möchte keinen belästigen, wenn's nich unbedingt sein muss. (Z. 349-351)

In Anbetracht der Tatsache, dass ihr soziales Netz sehr klein ist, wie aus der folgenden Aussage hervorgeht, sind der Angehörigenkurs und später die Gesprächsgruppe für Angehörige eine wichtige Quelle der emotionalen Unterstützung.

Interviewerin: Können Sie mir vielleicht Personen nennen, zu denen Sie im Moment die engste Beziehung haben?

Frau T.: Eigentlich nur zu meiner Schwester. Meine Mutter ist tot und man hat ja sonst keinen. Vielleicht noch 'ne Arbeitskollegin, wo man sich mal aussprechen kann. Aber sonst hab ich keinen. (Z. 488-492)

Allerdings fällt es ihr insgesamt schwer, emotionale Unterstützung anzunehmen, wie sich in folgender Aussage zeigt, da sie kaum Nutzen für sich darin erkennen mag:

Interviewerin: Gibt es denn in der derzeitigen Situation noch etwas, was Ihnen Trost gibt oder Hilfe?

Frau T.: Nee. Wie gesagt, ich hätte immer'n schlechtes Gewissen, wenn ich jetzt weg bin. Det muss man ja auch mit sich vereinbaren können.

Interviewerin: ... oder vielleicht bestimmte Menschen, die Ihnen Trost geben können oder ...?

Frau T.: Äh, det nützt ja alles nischt, det bleibt ja trotzdem wie gehabt - det nützt allet nischt. (Z. 483-487)

Immerhin nimmt Frau T. regelmäßig professionelle Unterstützung in Form eines Schiebedienstes in Anspruch, wobei sie aber bei den Spaziergängen mitgeht. Im 
Vordergrund steht nicht so sehr die zeitliche Entlastung, sondern vielmehr, dass ihr jemand die körperliche Anstrengung des Rollstuhlschiebens abnimmt, da sie häufig unter Rückenschmerzen leidet.

Frau T.: (...) Nun hamwa vom Sozial-Cop (Anmerkung: ABM-Projekt) einen Schiebedienst, die kommt einmal in de Woche so von zwei bis vier, (...) und da fahrn wa, da geh ich dann auch mit. Ich fahr immer im Park spazieren und anschließend gehn wa dann noch ins Zentrum, das geht dann nämlich den Berg hoch und ich kann nicht mehr so schieben. Und da ist das ganz gut, wenn wa schon da oben sind. Zurück geht's ja, weil's ja dann bergab geht. Es ist zwar nicht viel, wenn man gesund ist. Früher hab ich den Berg nie gemerkt, ne. Aber wenn man den schieben muss, nun is mein Mann ja nun auch 90 Kilo mittlerweile, weil er sich eben nicht bewegt und immer Appetit hat (...)(Z. 3240)

Dass Frau T. sich mit dem Schiebedienst Entlastung für ihren Rücken schafft, ist schon ein großer Fortschritt gegenüber früher, wie der folgende Interviewausschnitt zeigt:

Interviewerin: Sagen Sie dann auch Ihrem Mann ... also, dass sie jetzt Rückenschmerzen haben?

Frau T.: Ja, ja. „Ja dann musste mal wieder zum Arzt gehen.“ Det war früher so: Hab mir morgens 'ne Spritze geholt und nachmittags hab ich ihn im Park rumgeschoben. Det kann ich einfach nicht mehr. Ick hab jar nich mehr die Kraft und die Energie dazu, ich bin einfach kaputt. Det mürbt auch so, wenn man selber Schmerzen hat, wird man ooch giftig. Wenn Sie solche Schmerzen haben, denn is einem eigentlich allet zuviel. Und dann wird man auch giftig und böse. Det will ich eigentlich gar nicht, bin nich so'n Typ, aber det wird man automatisch. (Z. 410-416)

Frau T. sieht selber einen Zusammenhang zwischen ihrer gereizten Stimmung und den Forderungen ihres Mannes, möchte dies eigentlich verändern, weiß aber nicht wie. Dabei ist Frau T. durchaus in der Lage, zu formulieren, was ihr gut täte, das beinhaltet aber eine räumliche Trennung von ihrem Mann:

Interviewerin: Was könnten Sie sich denn für sich vorstellen, wo Sie wüssten, das würde Ihnen gut tun?

Frau T.: Ich möchte mal in Urlaub fahrn, mindestens 14 Tage inne Sonne und den janzen Tach nur mich. Morgens aufwachen und denken: Brauchst ja nur für dich. Wat machste heute? Und wenn ich vor Langeweile sterbe, aber ... Oder den ganzen Tag auf'm Stuhl sitze, aber nur mal ganz für mich alleine sein - det wär schön. (Z. 417-421) 
Die Vernachlässigung ihrer eigenen Bedürfnisse geht sogar soweit, dass sie in einer gesundheitlich kritischen Situation (sie bekommt eines Morgens plötzlich starkes Nasenbluten) versucht, sich allein zu behelfen. Als das Nasenbluten nicht nachlässt, wird sie schließlich nachmittags von der Feuerwehr ins Krankenhaus gebracht. Ihr Mann, der in der Zwischenzeit von der Nachbarin versorgt wird, dringt in einem Telefongespräch mit ihr ungeduldig auf ihre Rückkehr, sodass sie gleich nach Versorgung in der Notfallambulanz wieder nach Hause geht (Z. 308-339).

Ein weiteres Beispiel für die Negierung von Befindlichkeitsstörungen zeigt sich in dem folgenden Gesprächsausschnitt, in dem Frau T. körperliche Beeinträchtigungen beschreibt; zwar sieht sie einerseits einen Zusammenhang mit ihrer Belastungssituation, kommt andererseits aber doch zu dem Schluss, dass ihre Beschwerden auf ein Nahrungsmittel zurückzuführen sind, das sie nicht vertrage.

Frau T.: (...) Denn der Ärger macht einen ooch krank. Ich hab neuerdings immer Magenschmerzen, aber immer so ganz komisch, und ich krieg nicht raus, woran des liegt. Ich habs schon auf'n Kaffee geschoben oder auf gebratenen Speck. Ich hab jetzt so viel Pilze gemacht ... kommt nachmittags, geht urplötzlich los, geht bis hier hoch und denn piekt des bis hinten in die Seiten rein. Gestern Abend ... die Nieren, ich hab gedacht, die fliegen mir weg. Und morgens, wenn ich aufwache, ist das wieder gut. Aber det war so schlimm, ich denke: Du spinnst, det kann doch nich sein. Und morgens is det weg: Ob ich auch schon 'ne Macke habe? Manchmal fängt man an zu spinnen.

Interviewerin: Warn Sie denn schon mal beim Arzt?

Frau T.: Nee, nee. Ick muss erst mal rausbekommen, woher det kommt, was ich nich vertrage. Irgendwat muss det sein ... oder det Brot, det Schwarzbrot, det ess ick ja schon jahrelang. Aber irgendwas muss det sein und morgens // komisch, morgens hab ick det nie, fängt immer nachmittags an und abends is det so schlimm, geh ich meist schon um acht ins Bett. (Z. 513-525)

Dass ihre gesundheitlichen Probleme möglicherweise eine psychosomatische Ursache oder zumindest Komponente haben, scheint sie letztlich nicht wahrhaben zu wollen, da ein derartiges Eingeständnis zur Folge hätte, dass eine Veränderung ihrer Situation angezeigt wäre.

Führt man sich die starke Fixierung auf ihren Mann vor Augen, ist es umso erstaunlicher, dass Frau T. finanziell stets unabhängig von ihrem Mann war. Sie hat 
immer gearbeitet und ein eigenes Konto gehabt. Dies ermöglicht es ihr nun, in der Pflegesituation immerhin in einigen Bereichen ihre Interessen durchzusetzen und sich kleine Freuden zu gönnen (Friseurbesuch, neue Schuhe), ohne ein schlechtes Gewissen zu haben. Durch ihre finanzielle Unabhängigkeit sind somit bei grundsätzlicher emotionaler Abhängigkeit von ihrem Mann wenigstens in Teilbereichen Ansätze zur

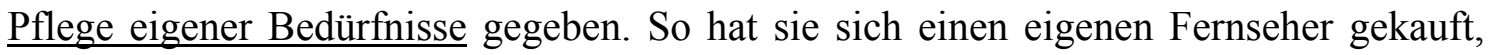
um damit unabhängig von den Fernsehgewohnheiten ihres Mannes zu sein und sich zuhause nicht mehr ,wie zu Besuch“ (Z. 537) zu fühlen:

Frau T.: (...) Und dann hab ich mir jetzt neuerdings 'nen Fernseher alleine gekauft, weil mein Mann, der hat das so furchtbar laut, ooch, der hat det so laut, det tut direkt weh, kann ick nich aushalten. Und wenn ich denn was sehen will, geh ich lieber in mein Bett, mach die Tür zu und denn kommt er um halb elf, kommt er denn, und denn zieh ich ihn aus, auf Toilette geht er vorher alleine, wäscht sich 'n bisschen und denn zieh ich ihn aus und denn ist er um halb elf im Bett, ja. Des hätt ich schon längst eher machen sollen mit dem Fernseher, da hab ich wenigstens bisschen Ruhe und habet für mich alleine. Ich guck ja ooch nich alles, manchmal les ich oder so. Aber schon det Gefühl, dat man seen Programm bestimmen kann. Wenn ich nämlich geguckt hab: Och, interessiert mich. BUMS, waret wieder weg mit der Fernbedienung, ne. Ja, hat mich meine Schwester druff gebracht, die hat gesagt: Bist du blöd, kauf dir doch einen alleine und dann kannste gucken, was du willst und brauchst da nich immer ... Ich war immer nur wie zu Besuch, is ja ooch nich der Sinn. (...) (Z. 526-537)

Auffällig ist, dass sie beim Durchsetzen eigener Interessen stets Anregung und Unterstützung von außen braucht, sei es durch ihre Schwester, wie in diesem Fall, oder im Fall des Familientreffens (siehe oben) durch die Ärztin und die Gesprächsgruppe. Wie wichtig ihr die Unterstützung durch die Gesprächsgruppe ist, zeigt sich auch darin, dass sie sich ihrem Mann gegenüber durchsetzen würde, wenn er ihr verböte, die Gruppe weiterhin zu besuchen:

Interviewerin: Ich frag jetzt mal so direkt nach, von dem, was Sie mir erzählt haben, wenn jetzt Ihr Mann sagen würde, dass er nicht mehr will, dass Sie hinkommen (Anmerkung: zur Gesprächsgruppe) oder so. Wie würden Sie darauf reagieren?

Frau T.: Det würd ick mir nich verbieten lassen (ENERGISCH), da wär ick denn bockig. Also, sowat gibt es nich, da wär's dann aus. Oder wenn er sagen würde: Brauchst nich zum Friseur gehn. Oder so. Oder: Gehst schon wieder? So was würd ich mir nie verbieten lassen, dazu bin ick ooch zu selbständig. Ich hab immer gearbeitet, wir hatten immer getrennte Konten gehabt. (...) Und dadurch 
bin ich finanziell auch unabhängig und hab mein eigenes Geld und da lass ich mir ooch nicht reinreden. Er sagt zwar: Hast schon wieder neue Schuhe, du hast doch den ganzen Schrank voll. Ich sag: Die ham mir aber gefallen, ob ichse brauch oder nich, die ham mir aber gefallen. (LACHEN) (...) (Z. 642-654)

\section{Biographische Aspekte, Beziehungsqualität und Pflegemotivation}

Eine Erklärung für die starke Identifikation mit ihrer Pflegerolle ist der biographische

Hintergrund von Frau T. Sie bezeichnet sich selbst als den Typ ,geborene Krankenschwester“ (Z. 446); sie musste sich bereits in jungen Jahren, von ihren Geschwistern in diese Rolle gedrängt, um ihre pflegebedürftige Mutter kümmern. Das Bedürfnis, für andere da zu sein und anderen zu helfen, ist so tief verwurzelt, dass sie sich nach dem Tod ihres Mannes sogar ehrenamtlich als „grüne Dame“ betätigen würde:

Frau T.: Ich hab mir des schon überlegt, wenn ich alleine wär, würd ich vielleicht ooch in'n Krankenhaus gehn oder Leute besuchen, die alleine sind. Wenn ich jetzt alleine wäre, Langeweile hätte oder irgendsowas. Wenn ich weiß, 'n andern, der sich dadrüber freut... manchmal sind's ja nur Kleinigkeiten oder Handlangersachen. Det würd ich gerne machen, ja. Aber jetzt im Moment nich, da hab ich mit meinem Mann genug zu tun. Wenn det vielleicht'n Mensch wär, der sich dolle darüber freut, man muss doch nichts dafür verlangen, ich möcht ooch nix dafür haben, sondern eben nur 'ne Anerkennung, dat det schön ist. Das würd ich gerne machen, aber im Moment nich. Det würde mein Mann auch nich//um Gottes Willen: „Kümmerst dich um andere, hast zu Hause genuch zu tun." Det würde der gar nich verstehn. (Z. 626-635)

Wenngleich sich Frau T. stark mit der Pflegerolle identifiziert, ist ihr Verhältnis zu ihrem Mann von Ambivalenz geprägt, wie die folgende Passage verdeutlicht:

Frau T.: (PAUSE, SEUFZER) Obwohl, alleine möcht ich auch nich sein, dann denk ich immer: Wenn's mal wirklich so kommt. Mein Mann is ja nun viel älter, wenn's mal wirklich so kommt, hätt man wirklich 'nen schlechtes Gewissen. Obwohl, wenn ich so richtig uff de Picke bin, manchmal hab ich schon jedacht: Bist du blöd, versaust dir deen janzet Leben, nimm dir doch einfach ne Wohnung alleine, kannst ja zu ihm kommen und ihm det machen, so wie er det will. Weil er zu Hause nichts machen lassen will, ja, darf keiner mal Fenster streichen: „Na jetzt doch nich.“ Nischt darf ick machen, mir sind einfach die Hände jebunden und det ... mir gefällt det so nich. Und denn wird man ooch unzufrieden, wenn einem det nich gefällt. Und det jeht ja nich um Geld, det könnten wa bezahlen und machen lassen. Ich mein, wenn man's nicht alleine kann, ich kann det nich alleine, muss man's machen lassen. Aber nee, det bringt Unruhe, nee, det muss nich sein. (PAUSE) Unruhe jehört nu mal zum Leben. 
Wenn man tot ist, brauch man nich mehr machen - so seh ick det, ne. (Z. 455467)

Die Vorstellung, dass ihr Mann irgendwann sterben wird, hat bei Frau T. ein schlechtes Gewissen zur Folge, was darin begründet sein könnte, dass dies durchaus einem uneingestandenen Wunsch entspringt. Der Gedanke, endlich von der Pflegesituation befreit $\mathrm{zu}$ werden und ihr Leben nach ihren eigenen Vorstellungen $\mathrm{zu}$ gestalten empfindet Frau T. als unzulässig, sodass er mit einem schlechten Gewissen abgewehrt wird. Das Gefühl, ihr Leben an ihren Mann zu „verschwenden“, formuliert sie indes nur indirekt und unterstreicht zuvor die Selbstverständlichkeit mit der sie die Versorgung ihres Mannes übernommen hat:

Interviewerin: War es für Sie selbstverständlich, die Pflege Ihres Mannes zu übernehmen?

Frau T.: (IM BRUSTTON DER ÜBERZEUGUNG) Ja. Hätt ich nie uff // wär ick NIE uff die Idee gekommen ... aber jetzt, wo die Jahre dahinschwinden ... Wie gesagt, ich habe Angst, ich bin selber alt und hilflos nachher, und denn war's des. Man hofft ja immer, dass man noch'n paar schöne Tage hat. (Z. 468472)

Bezeichnenderweise beantwortet sie die Frage nach positiven Aspekten der Pflege nicht direkt sondern mit einem pauschalen ,,ick mach det eigentlich gerne“.

Interviewerin: Die Situation ist ja auch für Sie sehr belastend und anstrengend: Finden Sie aber auch positive Dinge in der Pflege?

Frau T.: (ÜBERLEGT EINE WEILE:) Ick mach det eigentlich gerne. Wenn ich an meine Schwester denke, die sagt: Ich könnte meinem Mann nicht den Hintern abwischen. (...) Wenn ick andere höre ... 'ne Kollegin, die sagt: Nee, pflegen könnt ick keinen, da könnt ich hilfeschreiend wegrennen. Det kann auch nicht jeder. (Z. 442-449)

Aber die Freude an der Pflegerolle nimmt man ihr nicht wirklich ab, zu groß ist die Zwiespältigkeit gegenüber ihrem Mann. So ist während des Gesprächs immer wieder deutlich zu spüren, dass Pflichtgefühl das tragende Motiv für die Pflege ihres Mannes ist und nicht etwa tiefempfundene Zuneigung zu ihrem Mann. Dieses Pflichtgefühl ist gepaart mit einer Schicksalsergebenheit, die sich in ihrer Äußerung „Jetzt hab ich angefangen, nu sitz ich drin“ (Z. 399-400) wiederspiegelt. Der folgende Gesprächsausschnitt zeigt, wie sehr die Empfindungen ihrem Mann gegenüber 
abgestumpft sind, was sie als „wie abgestorben“ beschreibt. Die große gefühlsmäßige Distanz wird verschärft durch eine tiefe Kluft der Sprachlosigkeit zwischen beiden Partnern, was Frau T. mit einem resignativen Schulterzucken und der Bemerkung ,wat soll's" kommentiert:

Interviewerin: Gibt es denn auch Veränderungen in der Beziehung zu Ihrem Mann seit dem Schlaganfall?

Frau T.: (KOMMT UNMITTELBAR) Ja, so det letzte Jahr bin ich nich mehr so ... war sonst immer so lieber noch, ja. Kann det einfach nicht mehr, weeß nich, warum. Des is auf einmal da, wie abgestorben. (PAUSE) Is eben so, kann man nich erzwingen, muss von Innen kommen und ... Er merkt des wohl auch, aber er sagt ... spricht nich darüber. (SEUFZER) Ja, wat soll's. Wie gesagt, da kann einem keiner helfen. (Z. 473-478)

Ihre Hilflosigkeit dem Verhalten ihres Mannes gegenüber, äußert sich - außer in der so resigniert klingenden, zuweilen weinerlichen Stimme während des Gesprächs - auch in dem häufigen Nachsatz: „Weeß ooch nich, wie man das ändern könnte.“ (z. B. Z. 277278).

\section{Interaktionen zwischen Bewältigungsverhalten und Intervention}

Nachdem der vorige Abschnitt der ausführlichen Analyse des Bewältigungsverhaltens von Frau T. galt, folgt nun eine Beurteilung der Interventionsmöglichkeiten anhand ihrer Aussagen im Interview. Es wird außerdem diskutiert, inwiefern das Bewältigungsverhalten der Angehörigen die Effektivität der genutzten Entlastungsangebote beeinflusst.

Im Rahmen der Fallanalyse wurde deutlich, dass aufgrund der starken Patientenfixierung die Interventionsmöglichkeiten bei Frau T. begrenzt sind. Die mangelnde Reflektionsfähigkeit bzw. -willigkeit von Frau T. führt dazu, dass sie Widersprüchlichkeiten konsequent ausblendet und es somit vermeidet, ihre Situation in Frage zu stellen. Dies wäre aber eine notwendige Voraussetzung, um Entlastungsmöglichkeiten in Anspruch zu nehmen, die ihr einen Freiraum zur Pflege eigener Bedürfnisse verschaffen würden (z. B. Kurzzeitpflege oder Tagesstätte). Den Kursbesuch „erlaubt“ sich Frau T. nur, weil sie die Teilnahme ihrem Mann und wohl auch sich selbst gegenüber mit der Begründung legitimieren kann, dass sie dort 
Informationen erhielte, die ihm zugute kämen. Gleichwohl ist die tatsächliche Motivation für die Teilnahme am Angehörigenkurs eine andere als das Bedürfnis nach Information, nämlich vielmehr das Bedürfnis nach emotionaler Entlastung durch den Austausch mit anderen Betroffenen. Dies illustriert der folgende Gesprächsausschnitt, in dem Frau T. den vermittelten Sachinformationen einen eher geringen Stellenwert zubilligt:

Interviewerin: Welche speziellen Anregungen haben Sie (Anmerkung: im Kurs) dann für sich nutzen können?

Frau T.: Nee, eigentlich hiervon ... Sie meinen, ob ich was unternommen habe danach?

Interviewerin: Nee, ich mein jetzt von dem Angehörigenkurs: Ob sich dadurch auch die Pflegesituation bei Ihnen geändert hat, ob Sie bestimmte Sachen dann anwenden konnten, die Sie vorher nicht wussten?

Frau T.: Ja, wat war'n det? Nee, eigentlich wusst ich des vorher schon, so. Wie gesagt, sind immer wieder Kleinigkeiten noch, die man ja ooch lernt bei oder durch Zufall mal mitkriegt. Im Ganzen hat mir det sehr viel gebracht. Im Allgemeinen find ick det eben jut, na hauptsächlich auch, um mal unter Leuten zu sein, denen's genauso geht. Und ich geh gerne wieder hin, bin froh, dass es jetzt weitergeht (Anmerkung: mit der Gesprächsgruppe für Angehörige). (...) Is immer schön, man kennt die Frauen schon und kennt ooch ihre Sorgen. (Z. 578588)

Auch wenn sie den Angehörigenkurs positiv bewertet, wirken ihre Äußerungen zum Nutzen insgesamt eher dürftig und unreflektiert. Sie kommt über die oberflächliche Äußerung „Ick find det jut“ nicht hinaus. Das, was ihr gut tut, schöpft sie gewissermaßen 'ex negativo': anderen geht es noch schlechter.

Als sie nach Abschluss des Kurses noch weiterhin die Gesprächsgruppe besucht, ist ihrem Mann zunächst nicht klar, dass es in erster Linie um die emotionale Entlastung seiner Frau geht und daher erhebt er keine Einwände. Später hat sich ihre Teilnahme an der Gruppe so etabliert, dass er ihr die Teilnahme nicht ausredet:

Interviewerin: Wie war das dann mit der Gesprächsgruppe, als sie nach dem Kurs noch daran teilgenommen haben?

Frau T.: Ja, er hat das aber zuerst nich mitgekriegt. Er hat gedacht, des geht um IHN. Und jetzt hat er erstmal mitgekriegt, dass das ja für die Angehörigen ist, nich. Wie die damit umgehn. Um uns geht es ja eigentlich, nich um ihn als Patient, sondern um uns. Det hat er erst später mitgekriegt, det fand er wohl nich mehr so gut, hat aber nichts gesagt. (LACHEN) (Z. 607-611) 
Der Austausch mit anderen Betroffenen trägt entscheidend dazu bei, dass es ihr schließlich gelingt, in einigen - wenigen - Situationen ihre eigenen Bedürfnisse mehr wahrzunehmen. So engagiert sie einen Schiebedienst für ihren Mann und verbringt ein Wochenende allein mit ihren Geschwistern. Auch wenn sich in ihrem Verhalten erste Ansätze zeigen, eigene Bedürfnisse stärker wahrzunehmen, in den Belastungswerten zeigen sich im zeitlichen Verlauf kaum positive Veränderungen. Es ist lediglich eine Abnahme der Angstsymptome $\mathrm{zu}$ verzeichnen, wobei das Angstniveau zum vierten Messzeitpunkt immer noch ein pathologisches Ausmaß erreicht. Zöge man lediglich die Belastungswerte zur Beurteilung der Effektivität des Kurses bei Frau T. heran, fiele die Bewertung negativ aus. In Anbetracht der Befunde aus der vertiefenden Fallanalyse zeigt sich jedoch, dass der Kurs für Frau T. eine wichtige Weichenfunktion hatte. Zum einen trug der Kurs sicherlich zu einer emotionalen Stabilisierung bei, zum anderen führte die Teilnahme zu ersten Veränderungen auf der Verhaltensebene.

Ein Jahr nach Durchführung des Interviews verstarb Herr T. Seine Frau kam noch dreimal nach seinem Tod in die Angehörigengesprächsgruppe. Den Tod ihres Mannes erlebte sie wie eine Befreiung und machte sich mit Tatkraft daran, aufgeschobene Träume zu verwirklichen. Unter anderem kaufte sie sich eine neue Küche sowie neue Einrichtungsgegenstände und gestattete sich einen sechswöchigen Urlaub.

\subsubsection{Frau M.: „Man muss einfach mit dem Hier, Heute und Jetzt umgehen lernen"}

Das Interview mit Frau M. fand im September 1998 statt. Herr M. ist zu dem Zeitpunkt 62 Jahre alt, Frau M. 64. Sie sind seit 36 Jahren verheiratet. Sie haben einen Sohn und eine Tochter sowie eine Enkeltochter. Herr M. war beruflich als Verwaltungsangestellter im Bezirksamt in der Abteilung Bildung tätig, bevor er aufgrund eines Rückenleidens 1996 frühpensioniert wurde. Frau M. ist gelernte Verkäuferin und arbeitete zeitweilig in einem Modefachgeschäft. Die Eheleute leben in einer 4-Zimmer-Wohnung in einem Innenstadtbezirk. 
Herr M. erlitt im Juli 1997 einen linkseitigen zerebralen Insult. Bis September 1997 befand er sich in einem Akutkrankenhaus, danach kam er in die Tagesklinik. Im Winter 1997/98 kam es jedoch zu einer Lungenentzündung, sodass ein erneuter stationärer Aufenthalt notwendig wurde. Es folgte eine Schilddrüsenerkrankung, die im März 1998 eine Operation erforderlich machte. Im Anschluss konnte er weitere 7 Wochen in der Tagesklinik therapiert werden. Seit dem Schlaganfall leidet Herr M. an einer Halbseitenlähmung rechts, an einer Aphasie und leichten neuropsychologischen Störungen, vor allem in den Bereichen Aufmerksamkeit und Konzentration, Rechenleistungen sowie visuell-räumliche Fähigkeiten. Als Risikofaktoren liegen bei Herrn M. ein Hypertonus sowie eine Hypercholesterinämie vor.

Wie aus Tabelle $17 \mathrm{zu}$ ersehen, finden sich bei Frau M. zum ersten Messzeitpunkt der Fragebogenstudie ein deutlich erhöhtes Ausmaß an Angstsymptomen und an körperlichen Beschwerden. Das Ausgangsniveau ist damit etwa mit Frau T. vergleichbar, wenn auch die Werte in der Allgemeinen Depressionsskala bei Frau M. noch unterhalb des auffälligen Bereiches liegen. Beim letzten Messzeitpunkt liegen jedoch bei Frau M. alle Werte wieder im Normbereich, während bei Frau T. zum Teil ein Anstieg der Werte beobachtbar war. 
Tabelle 17: Ausprägung von Depression, Angst und körperliche Beschwerden zu den 4 Messzeitpunkten bei Frau M.

\begin{tabular}{lcccc}
\hline Variable & $\begin{array}{c}\text { Meßzeit- } \\
\text { punkt 1 }\end{array}$ & $\begin{array}{c}\text { Meßzeit- } \\
\text { punkt 2 } \\
(+5 \text { Monate })\end{array}$ & $\begin{array}{c}\text { Meßzeit- } \\
\text { punkt 3 } \\
(+\mathbf{1 0} \text { Monate })\end{array}$ & $\begin{array}{c}\text { Messzeit- } \\
\text { punkt 4 } \\
(+\mathbf{1 3} \text { Monate })\end{array}$ \\
\hline Depression (ADS) & 17 & 14 & 17 & 15 \\
Angst (SAS) & $\mathbf{4 3}$ & 34 & $\mathbf{3 7}$ & 32 \\
körperliche Beschwerden (B-L) & $\mathbf{2 8}$ & $\mathbf{2 7}$ & $\mathbf{2 8}$ & 20 \\
& & & & \\
\hline
\end{tabular}

Anmerkungen: ADS: Allgemeine Depressionsskala; SAS: Self-rating Anxiety Scale; B-L: BeschwerdenListe. Fettgedruckte Werte kennzeichnen solche, die oberhalb des Cutoffs für die entsprechende Variable liegen und deuten auf Auffälligkeiten im jeweiligen Bereich hin. Die Grenzwerte liegen bei: ADS: $\geq 23$; SAS: $\geq 36$; B-L: $\geq 25$.

\section{Pflegerische Aufgaben}

Im Gegensatz zu Frau T. ist Frau M. in der Lage, ihr pflegerisches Engagement an die Fähigkeiten ihres Mannes anzupassen. Zum ersten Messzeitpunkt (September 1997) gab sie noch im Fragebogen an, sechs Stunden pro Tag für alle Versorgungstätigkeiten zu benötigen. Der Barthel-Index lag bei 45 Punkten. Herr M. brauchte Hilfe beim An- und Ausziehen, bei Toilettengängen, bei der Körperpflege sowie beim Laufen. Der Umfang der Pflegetätigkeiten nimmt dann kontinuierlich ab: zum vierten Messzeitpunkt, fast eineinhalb Jahre nach dem Schlaganfallereignis, beträgt der Unterstützungsaufwand pro Tag nur noch zwei Stunden, der Barthel-Index liegt bei 90 Punkten. In der folgenden Textpassage finden sich ebenfalls Hinweise, dass Frau M. parallel zu den Fortschritten ihres Mannes ihre pflegerischen Tätigkeiten zurückgenommen hat:

Interviewerin: Lassen Sie uns beginnen mit Ihrer derzeitigen Alltagssituation und vielleicht können Sie einmal einen typischen Tagesablauf von sich schildern.

Frau M: Jetzt von diesem Zeitraum? Also, der sieht jetzt schon wieder viel glücklicher und nicht gar so strapaziös aus wie zu Beginn der Krankheit meines Mannes. Er kann sich nämlich inzwischen alleine waschen, was sehr wertvoll ist. Also, ich mach dann den Rücken, da kommt er schlecht ran, aber ansonsten geht alles wieder alleine. Und er muss auch nicht mehr SITZEN. Im Anfang 
musste ich ihn ja hinführen zum Waschbecken und dann hat er mit der einen Hand so'n kleines bisschen versucht, sich zu waschen, aber den Löwenanteil habe ich gemacht, weil // insofern sieht es jetzt sehr viel günstiger aus und ich brauche nicht mehr so viel Zeit und nicht mehr so viel Kraft, ja. Waschen, Rasieren, dann kommt Anziehen an die Reihe, da sucht er sich auch wieder seine Sachen, die er anziehen möchte, selber aus. (...) (Z. 1-11)

Im folgenden Gesprächsausschnitt bekennt Frau M. offen, dass sie die pflegerischen Tätigkeiten zu Beginn als große Belastung empfand. Vor allem störte sie der zeitliche Aufwand, aber auch das Gefühl der Unabkömmlichkeit:

Frau M.: (...) Und wie gesagt, ich muss ja auch nicht immer dabei sein und was ... also beim Baden schon, aber sonst beim Waschen Rücken, aber sonst kann ich rausgehen. Ich musste ihn ja zuerst ja immer rasieren und die Zähne putzen. Ich kann ihnen gar nicht sagen, wasses für 'ne Katastrophe ist, wenn man 'nem Menschen die Zähne putzen muss, der des'n Leben lang selbst gemacht hat. Ähm, und dann macht man des wahrscheinlich nicht richtig, man piekst dann da rum und dann tut's weh und dann hat er sich aufgeregt, so gut er konnte, dass ich ihn wieder irgendwo gepiekst habe mit der Zahnbürste. Des macht er ja alles alleine, er kann zwar nicht mit der rechten Hand die Zähne putzen, aber er kannse mit der linken so schön putzen, mit der rechten geht das Rasieren schon, aber ooch nicht hundertprozentig. Aber des sind alles so Sachen, die musst ich ja zuerst komplett machen.

Interviewerin: Und wie ging es Ihnen damit?

Frau M.: Das war 'ne ziemliche Belastung. Also, morgens eineinhalb Stunden bis er erst mal fertig war, abends weniger, weil man vielleicht nich ganz so viel an Wäscherei macht, aber Zähneputzen blieb. Und ja, man muss auch immer da sein, man kann sich im Grunde genommen nicht mal'n Schnupfen leisten, ja. Man muss also immer fit sein, auch wenn einem nach Fitsein manchmal nicht zumute ist. (Z. 230-246)

\section{Verwendete Bewältigungsstrategien}

Insgesamt verfügt Frau M. über eine große Anzahl von Bewältigungsstrategien, sie wendet 8 patientenzentrierte und 14 ichzentrierte Strategien an. Bei den patientenzentrierten Bewältigungsmechanismen fällt auf, dass Frau M. im Gegensatz zu Frau T. die Selbständigkeit ihres Mannes an vielen Stellen fördert, indem sie ihn z. B. in Haushaltstätigkeiten einbezieht oder ihn alleine zu den Therapien fahren lässt, nachdem sie den Weg gemeinsam mit ihrem Mann geübt hat (Förderung der Selbständigkeit): 
Frau M.: (...) Und dann kommen die Vormittage, sind meistens belegt mit Therapien, die er mittlerweile außer Haus bekommt, weil er eben wieder hergestellt ist, kommt niemand mehr ins Haus. Und äh, wir haben zu Anfang die Strecken zusammen geübt, einmal muss er von uns, wir wohnen am JakobKaiser-Platz, da muss er einmal nach Siemensstadt fahrn mit'm Bus und einmal mit der U-Bahn, Bus U-Bahn kombiniert, bis zum Theodor-Heuss-Platz. Das in Siemensstadt ist die Krankengymnastik, Theodor-Heuss-Platz ist die Ergotherapie. (Z.11-17)

Dabei fiel es ihr zu Beginn sehr schwer, ihren Mann allein zur Therapie gehen zu lassen, wie sie in der Gesprächsgruppe zugibt. $\mathrm{Zu}$ Beginn wendet Frau M. die Bewältigungsstrategie Kontrolle noch häufig an und kontrolliert ihren Mann durch ihre ständige Anwesenheit sehr stark, aus Angst, dass er beim Laufen fallen könnte:

Frau M: Ich muss dazu sagen, dass ich eigentlich im Grunde genommen ÜBERHAUPT KEINE Vorstellung hatte, was da auf mich zukommt, ja. Also sicher, zuerst, als mein Mann jetzt dann nach Hause kam im Oktober letzten Jahres, nachdem er ja noch 14 Tage wegen der Lungenentzündung im Krankenhaus war, das war 'ne ganz schlimme Zeit. Ich musste praktisch JEDEN SCHRITT beaufsichtigen, weil des SO SCHLECHT ging, dass ich immer dachte: Beim nächsten Schritt fällt er hin. (Z. 168-173)

Zwar gibt sie in einer weiteren Textstelle zu, nach wie vor Angst vor einem Sturz ihres Mannes zu haben. Dies hat jedoch keine aktive Einschränkung ihres Mannes mehr zur Folge. Stattdessen überträgt sie die Verantwortung ihrem Mann und instruiert ihn lediglich, aufzupassen und Hindernisse selbständig aus dem Weg zu räumen:

Frau M: (...) Und jetzt, wenn mein Mann jetzt stolpert ... also, ich da bin ich fast hysterisch, dass er hinfallen könnte. „Mach dir doch nicht so viele Gedanken!““ Ich sag dann: Ich kann des gar nich // räum dir bloß immer alles aus'm Weg hier in der Wohnung, steig nicht irgendwo drüber weg, pass an Treppen und Bordsteinkanten und so auf. Also, des is so'n bisschen // des Nervenkostüm noch'n bisschen ruiniert bei mir. (Z. 368-372)

Um das Anziehen zu trainieren, bezieht Frau M. ihren Sohn ein, der solange mit dem Vater übt, bis er sich selbständig wieder ankleiden kann (Co-therapeutisches Engagement):

Frau M.: (...) auch nachher mit dem Anziehen. (...) Also, des hat er an dem Tag überhaupt nicht hingekriegt, ja. Also, die Folge davon: Hab ich ihm an dem Tag 
das Hemd angezogen und denn kam unser Sohn an dem Tag noch. Und mich beschäftigt unablässig der Umstand, dass Papa das Unterhemd nicht angekriegt hat. Also, können wa des nich mal so machen: Du setzt dich daneben, dass er dieselben Bewegungen macht. Und des klappte, des war von Erfolg gekrönt, nich. Und denn saßense beide da, Oberkörper nackt, 'n Unterhemd vor sich. Und denn hat mein Mann immer geguckt, wie mein Sohn des macht: „Mach doch bitte nochmal." Sprechen ging jetzt noch nich so gut, wie ich Ihnen des erzählt habe. Aber er hat sich verständlich gemacht, dass er das nochmal machen soll und nach'm dritten Mal hat mein Mann das wirklich begriffen. Und denn ging des auch. (S. 553-569)

Frau M. äußert ihrem Mann gegenüber häufig Zuspruch, um ihn aufzumuntern und zu motivieren. Sie zeigt dabei sehr viel Verständnis und Geduld für seine psychische Verfassung:

Frau M.: (HOLT LUFT) Ja, manchmal fehlen die Kräfte, die körperlichen Kräfte noch irgendwas an den Tagen, wo Therapie ist, noch nebenher was zu unternehmen und manchmal ist er von der Gemütsverfassung irgendwie beeinträchtigt, ja. Dann issa jeknickt, dass nich mehr alles so is wie früher. Dann muss ich ihn immer ja so'n bisschen aufbaun, denn er hat ja bei dem Einbruch in seine Gesundheit oder in sein L..(Leben?), trotz alledem noch Glück gehabt, er konnte sich nich rühren, nich bewegen, nich sprechen. Er kann wieder laufen, wenn's auch langsam und bedächtigt geht, ohne Stock, ohne Hilfe. Zuerst mussta im Rollstuhl sitzen, da ging gar nichts, da hat er grade so die zwei Schritte zum Rollstuhl geschafft. Und dagegen is ja jetzt, dass er wieder laufen kann, det is doch'n WUNDER. Und irgendwie muss ich ihm det dann zu Bewusstsein bringen, denn irgendwo is ja // na ja, is 'ne Einschränkung da, wie gesagt beim Sprechen, beim Laufen, und auch das Schreiben klappt nicht so. Er hat Tage bei, das geht auch langsam, da schreibt er drei, vier Zeilen und an einem andern Tag kriegt er kaum seinen Namen hin und dann issa natürlich ein... // dann issa gemütsmäßig einfach so belastet, dass ich ihm dann immer wieder gut zureden muss, WIE GUT es ihm doch eigentlich geworden ist. Aber ich kann das schon verstehen, es überkommt ihn halt immer wieder. (Z. 35-49)

Es findet sich im Interview kein direkter Hinweis auf den Einsatz der Bewältigungsstrategie Stärkung des Kompetenzerlebens. Bei den Treffen der Gesprächsgruppe schilderte Frau M. jedoch wiederholt Situationen, in denen sie ihren Mann in Entscheidungen einbezieht und ihn um Rat fragt. Dies setzt sie bei einigen Gelegenheiten bewusst ein, um ihm das Gefühl des Gebrauchtwerdens zu vermitteln. Zum Beispiel nimmt sie ihren Mann beim Kauf einer neuen Couchgarnitur mit und lässt sich von ihm beraten, obwohl sie nach eigener Aussage ohne ihn schneller und unkomplizierter zu einer Entscheidung gelangt wäre. 
Anders als Frau T. bemüht sich Frau M. nicht übermäßig, psychische Belastungen fernzuhalten (Fernhalten psychischer Belastungen). Zwar hält sie sich in Auseinandersetzungen mit ihrem Mann mehr zurück als früher, äußert aber dennoch durchaus ihren Unmut:

Frau M.: Na, wir gehen vielleicht - im übertragenen Sinne - behutsamer miteinander um, ja. Des gibt ja auch Situationen, wo man sich zankt, nich. Man ist ja jetzt anner Auseinandersetzung überhaupt nicht mehr interessiert. Also beißt man sich lieber mal auf die Lippen oder sagt: Also, nu' hör mal, so geht // hat mir det jetzt nich gefallen, aber Schwamm drüber. (Z. 502-505)

Indem Frau M. intensiven Kontakt mit den behandelnden Therapeuten ihres Mannes pflegt und das Angebot der Hospitationen in der Tagesklinik nutzt, sorgt sie für optimale Therapiebedingungen (Sicherstellen optimaler Behandlungsbedingungen). Auch die Verschreibung ambulanter Therapien nach dem Tagesklinikaufenthalt ist ihr ein wichtiges Anliegen.

Die Anpassung der Lebensumwelt stellt bei Frau M. ebenfalls eine sehr wichtige Bewältigungsstrategie dar. Sie bemüht sich sehr, die Wohnung behindertengerecht zu gestalten, und nimmt dafür manche Unannehmlichkeit wie Verzögerung der Installation und Auseinandersetzungen mit der Wohnungsbaugesellschaft in Kauf.

Frau M.: (...) Und dann konnte er sich ohne Hilfe nicht aufs/auf die Klobrille setzen. Und nun ist des Klo in so 'ner kleinen Nische, an der einen Seite ist 'ne Rohrverkleidung, da sind die Versorgungsrohre hinter, an der andern Seite ist die Wand. Und die Krankenkasse, die BKK, hatte denn gesagt, sie wird'n Griff installieren lassen. Aber mein Mann war denn schon längst zu Hause, ja. Er sollte auch zu Hause sein, so war des damit die richtige Richtung. Aber er war dann schon zu Hause und drei Wochen später kam da erst jemand. (...) Des warn so Schwierigkeiten, die VÖLLIG auf die Nerven gingen, weil man einerseits // und denn dauerte des auch noch so lange. Ich war jeden Tag böse, warum des noch nicht installiert ist, nich. Nachdem des denn da war, war des alles kein Problem mehr. (...) Genau mit dem Badelifter: Es dauerte und dauerte und dann musste ich die Gesellschaft noch miteinbeziehen, (...) $\mathrm{Na}$ ja und dann funktionierte des und des is 'ne feine Sache mit dem Lifter. Also, in die Badewanne steigen, das könnte mein Mann ohne Schwierigkeiten noch immer nicht. Also, insofern ist des mit dem Badelifter 'ne riesige Hilfe. (Z. 175-209) 
Hilfsmittel werden als große Erleichterung im Alltag angesehen und als ein wichtiger Beitrag für den Patienten zur Wiedererlangung der Selbständigkeit:

Frau M.: (...) Vielleicht wird's noch besser - nach den guten Erfolgen bin ich da ja guter Dinge und denke: Also, er kriegt da ja auch noch'n bisschen mehr Sicherheit, ja. Und insofern sind diese Sachen // sie ham ja auch hier in der Tagesklinik auch so'n Lifter, denn hatten wa uns // also, ehe unser Lifter installiert war, hab ich den angeguckt. Also, diese Sachen sind ja unbezahlbar im Grunde genommen für den Patienten, ja. (Z. 222-226)

Frau M. nutzt anders als Frau T. vielfältige Quellen zur Suche nach Hinweisen und Ideen (Informationssuche), die das Leben mit der Erkrankung ihres Mannes erleichtern. Hospitationen in der Tagesklinik bei den verschiedenen Therapien haben den Zweck, mehr Informationen über die Erkrankung und über Bewältigungsmöglichkeiten zu gewinnen. Frau M. gelangt dadurch $\mathrm{zu}$ einer realistischeren Einschätzung der Einschränkungen ihres Mannes und auch zu mehr Sicherheit im Umgang mit ihrem Mann, wie sie in der folgenden Textpassage angibt:

Frau M.: (...) da hat der Logopäde dann mit ihm // da kriegte er dann so'n Kästchen mit Buchstaben, dann sollte er immer so Buchstaben einordnen. Da war denn ein Wort bei, „Strunk“", und des konnte er nicht in Griff kriegen, da hat er immer statt Strunk hinten vorm K noch'n $\mathrm{R}$ gelegt. Ich hab den Mann bewundert, ich hab jedacht: Des kann nich wahr sein. Der macht des immer wieder so lange, bis mein Mann des ungefähr drauf hatte. (...) Aber des waren dann solche Ausfallerscheinungen, es ging dann einfach nicht und urplötzlich war des wieder da. Wahrscheinlich nur durch dieses ständige daran arbeiten, denk ich mal. (...) Der Herr B. hatte dann bald zu mir gesagt: Kommense doch, hörense doch mal zu, wie des geht. Und dann hatte er mir gleich zu Anfang'n Merkzettel mitgegeben, dass man nicht etwa mit dem Aphasiker nun rumschreit und denkt, der ist schwerhörig und kann des deshalb nicht. Ja, unwillkürlich wird man immer lauter, wenn keine Reaktion kommt. Ja, diese Dinge waren SO hilfreich, ja, was man da von den Therapeuten an die Hand bekommen hat, auch nachher mit dem Anziehen. Also, immer von der betroffenen Seite zuerst anziehen, nich. (Z.538-554)

Auch durch die Teilnahme am Angehörigenkurs erfährt sie viele hilfreiche Informationen, die ihr bei der Bewältigung des Alltags dienen:

Frau M.: Aus dem Grunde hab ich den Kurs gemacht und hab alles nach Möglichkeit in mich aufgesaugt, dass ich im Zweifelsfalle immer darauf 
zurückkommen kann, ja. Also, ich könnte nur jedem empfehlen, so'n Kurs zu besuchen. Es is nich alles für einen zutreffend, is auch richtig. Man hört ja immer wieder: Ach, da kann ich ja nich alles gebrauchen. Und was die da noch alles erzählen ... is Quatsch, ist in jeder Stunde irgendetwas bei gewesen, was ich für unsere Situation habe entweder praktisch oder gedanklich verwenden können, nich. (Z. 681-687)

Interviewerin: Sie konnten auch vom Angehörigenkurs Dinge mit in die Pflegesituation nehmen, \#dass sich da was verändert hat\#?

Frau M.: \#Ja.\# Ja, also speziell jetzt nun, als mein Mann // diese Waschsituation zu Hause: Des gibt so kleine Tricks und Kniffe, die einem hier vermittelt wurden, damit kann man besser umgehen. Und beim Anziehn, dass man den Betroffenen von der betroffenen Seite anzieht und nicht andersrum, weil man ihm vielleicht sonst noch den letzten Rest hieroben anner Schulter verschiebt. Also wenn die rechte Seite betroffen ist und man zieht von links an, muss der Arm ja ziemlich weit nach hinten ... geht gar nicht. Oder wie man den Kranken nach Bobath lagert. Des hat mein Mann alles SEHR WOHLWOLLEND empfunden, ja, sehr hilfreich, nich (...). (Z. 701-710)

Des Weiteren dient die zeitweilige Unterstützung durch eine Hauskrankenpflege nicht nur zur Entlastung, sondern Frau M. eignet sich quasi durch Lernen am Modell (Informationssuche) mehr Sicherheit bei der Pflege an:

Frau M.: Zuerst hatt ich auch noch - nach der Lungenentzündung - morgens immer 'ne Hauskrankenpflege und diese Frau, die da kam, die hat mir des denn auch alles noch besser zeigen können, als wenn ich mich da selber versucht hätte. Sie hatte die ersten Male meinen Mann gebadet. (...) (Z. 209-212)

Frau M. verfügt über ein großes Repertoire an ichzentrierten Bewältigungsstrategien, die primär der Verbesserung der eigenen Befindlichkeit dienen. Dazu zählt unter anderem Akzeptieren; es gelingt Frau M. gut, das Unabänderliche anzunehmen, wenngleich sie angibt, zunächst mit dem Schicksal gehadert zu haben. Aber sie hält sich nicht lange damit auf, über das „Warum und Weshalb“ zu grübeln, sondern nimmt die Situation wie sie ist und versucht diese bestmöglichst zu bewältigen. So spricht aus der folgenden Textpassage eine große Gelassenheit:

Frau M.: Aber im Grunde genommen zuerst hab ich - natürlich - gehadert mit dem Schicksal, dass nun sowas passiert. Dann fragt man sich: Wie konnte sowas passieren? Gewiss, vielleicht gab's des eine oder andere Vorzeichen, hat man aber bestimmt nicht registriert. Und dann hab ich festgestellt, also dieses Grübeln über die Ursachen „Wieso, warum und nun grade ich?“, das bringt 
einem ÜBERHAUPT NICHTS. Man muss dann einfach die Gegebenheiten so nehmen, wie sie sind und versuchen, des Beste draus zu machen - kostet viel Kraft, kostet körperliche Kräfte, kostet nervliche Kräfte, aber was anderes bringt einen nicht weiter. Man kann jetzt nicht hadern, was vielleicht vor 10 Jahren nicht beachtet wurde oder was - geht nicht. Man muss einfach mit dem Hier, Heute und Jetzt umgehen lernen. Is ooch nicht einfach, ja. Aber ist die einzige Möglichkeit, um damit klarzukommen. Ich kann jetzt nicht darüber nachgrübeln, was ist gewesen und was wäre, wenn dies und jenes nich jewesen wäre - geht nicht, haut nich hin. (Z. 433-442)

Grundsätzlich hat Frau M. eine sehr positive Einstellung zur Erkrankung ihres Mannes. So vermag sie sogar eine positive Veränderung seit dem Schlaganfall zu benennen (Gutes im Negativen sehen):

Interviewerin: Gibt es denn Veränderungen in Ihrer Beziehung im Vergleich zu früher?

Frau M.: Jetzt zwischen meinem Mann und mir?

Interviewerin: Genau - also seit dem Schlaganfall sag ich jetzt mal.

Frau M.: Na, wir gehen vielleicht - im übertragenen Sinne - behutsamer miteinander um, ja. (Z. 499-503)

Auch an anderer Stelle wird ihre grundsätzlich positive Einstellung gegenüber ihrer Situation deutlich. Im folgenden Gesprächsausschnitt betont Frau M., dass das Schicksal es insgesamt gut mit ihnen meinte und sie den Krankheitsverlauf als sehr positiv bewertet. Diese Einstellung steht im großen Kontrast zu den Äußerungen von Frau T., die insgesamt vom Gefühl der Resignation geprägt sind.

Frau M.: (...) Aber irgendwie hatten wir ja Glück, Riesenglück. Ich seh det einfach als riesigen Glücksumstand, DASSER wieder soweit hergestellt ist, DASSER laufen kann, DASSER schreiben kann, DASSER sprechen kann. (Z. 627-629)

Frau M. geben außerdem ihre religiösen Überzeugungen einen gewissen Halt und Trost (Religiöse Bewältigung), wie sich in der folgenden Äußerung zeigt:

Interviewerin: Gibt es in der derzeitigen Situation etwas, was Ihnen Trost gibt oder Ihnen hilft?

Frau M.: (ÜBERLEGT) Na ja, sagen wa mal so: Man hat ja vielleicht immer mal wieder den Hang anzunehmen, dass es'n lieben Gott gibt, ja. Und des is natürlich auch'n Umstand, der einem weiterhilft, ja. (PAUSE) Ich bin kein 
fleißiger Kirchgänger, so ist des nicht, aber ick bin eigentlich so erzogen worden, dass ick mich immer wieder damit beschäftigt habe, und irgendwie ... doch, das hat mir geholfen. Na ja, und dann immer wieder meine Familie, ja ... und selbst mein Mann, trotz seiner Krankheit, ja. (Z. 650-654)

Frau M. führt die Fortschritte ihres Mannes ganz selbstverständlich auch auf ihr eigenes aktives Bemühen zurück, woraus sie eine gewisse Befriedigung und Stärke zieht (Vergegenwärtigen der eigenen Erfolge). Diese Einschätzung wird von den Therapeuten und dem behandelnden Arzt geteilt:

Frau M.: (...) Aber ich hätte da gar nicht anders gekonnt, als mich um ihn zu kümmern, und ich muss - vielleicht riecht das jetzt so'n bisschen nach Eigenlob - aber ick bin auch jeden Tag im Krankenhaus gewesen, von Anfang an. Ich glaube, da fehlt nich ein Tag. Und äh, die Therapeuten hier und auch der Doktor H., die ham jesagt, dass hat meinem Mann sehr viel gebracht, sehr viel weiter geholfen. Und als mein Mann dann mit dieser schrecklichen Lungenentzündung im Virchow lag, da bin ich auch jeden Tag hingegangen. (Z. 354-359)

Durch temporale Vergleiche macht sich Frau M. die Fortschritte, die ihr Mann erreicht hat, immer wieder bewusst. Gleichzeitig zeigen ihre Äußerungen, dass sie sehr realistische Erwartungen an den Krankheitsverlauf stellt, da sie davon ausgeht, dass besonders im Bereich des Sprechens Defizite bleiben werden. Diese beurteilt sie jedoch als nicht sehr gravierend.

Frau M.: Er hatte zu Anfang auch Logopädie, da issa auch wieder gut herjestellt worden, aber mehr // ich meine, er spricht ganz gut, außer wenna abgespannt ist, dann fängt er an zu nuscheln, dann geht manches so'n bisschen unter, das is wohl nicht mehr hinzukriegen. Aber es ist'n Riesen-Fortschritt erzielt worden und wenn nur das bisschen Nuscheln bleibt, sind wa ja glücklich und zufrieden, ja. (Z. 17-21)

Neben temporalen Vergleichen zieht Frau M. auch soziale Vergleiche und relativiert somit ihr eigenes Schicksal. Ähnlich wie bei Frau T. bietet vor allem der Angehörigenkurs gute Möglichkeiten, soziale Vergleiche herzustellen:

Frau M.: (...) Also, manche Frauen (aus dem Angehörigenkurs) haben mir wahnsinnig leid jetan. Ich hab immer gedacht, da is deine Situation noch/noch gut gegen. Eine Frau war hier, ich glaub', da is der Mann schon über zehn Jahre behindert, die haben da in der Nähe inner Behindertenwohnung // ooch so, wo man sich dann nachts durch Knopfdruck Hilfe holen kann und sowas. Also, da 
hab ich gedacht: Des is ja schlimm. Da fängt man dann nämlich an zu rechnen und rechnet, wann dem Mann das wiederfahren ist. Und die Frau, die muss nun so viele Jahre schon dafür grade stehn, dass alles läuft. Die kann sich // ja, ick denk immer, man kann sich dann gar keine eigene Einbuße leisten. Und die Frauen waren auch erstaunlicherweise alle gut drauf, ja. (Z. 727-735)

Die Bewältigungsstrategie Distanzierung verhilft Frau M. zu einer relativ stabilen psychischen Situation. Die Möglichkeit, dass sie selbst erkranken könnte, schiebt Frau M. bewusst von sich. Dieses Ausblenden irrationaler Ängste verhelfe ihr zu einem größeren Durchhaltevermögen, wie sie in folgender Äußerung sagt:

Frau M.: (...) Ganz davon abgesehen, dass ich bei all den Dingen immer dran gedacht habe, was ihm passieren kann, und nie dran gedacht habe, dass mir ja auch was hätte über den Weg laufen können, oder noch kann. Das ist 'ne merkwürdige Einstellung, nich? Aber wahrscheinlich hat mir // gibt diese Einstellung mehr Durchhaltevermögen, dass man immer denkt, einem selbst kann überhaupt nichts passieren, nich. (Z. 390-395)

Anders als Frau T. nimmt sich Frau M. im Alltag stets bewusst Zeit für Regenerationsphasen (Erholung). Sehr erholsam sind für sie kurze Schlafpausen, auch monotonen Haushaltstätigkeiten gewinnt sie ein gewisses Entspannungsgefühl ab.

Interviewerin: Was tun Sie für sich, wenn es Ihnen schlecht geht? (PAUSE) Fällt Ihnen da vielleicht ein konkretes Beispiel ein? Was Sie so erlebt haben, was sie in so einer Situation getan haben?

Frau M.: Also, ich suche einfach dann immer automatisch immer Ruhe - wenn's möglich ist - und 'ne Stunde Schlaf. Komischerweise hat mir des immer weiter geholfen. Ich bin dann manchmal aus'm Krankenhaus gekommen und war kaum noch in der Lage 'nen Schritt zu tun. Ich war gar nich mal müde (...) Oder wenn ich so kaputt war, einfach // also, ich kann auch auf'm Teppich schlafen, ich lieg auf'n Teppich, roll mich zusammen und schlafe. Det is vielleicht'n Glücksumstand für mich, ja. Ich muss mich nicht mal zudecken, Hauptsache ich liege erst mal. Ich werde dann zwar wach, wenn mir kalt ist, aber ich habe des erstmal wieder erreicht, dass ich zu mir gekommen bin.(...) Also einfach versuchen, 'nen Zipfel Ruhe zu kriegen. Das war auch, als mein Mann dann zu Hause war und ich dann so häufig nachts aufstehen musste. Da hab ich dann manchen Morgen gedacht: Also, wenn ich jetzt doch noch 'ne Stunde liegen könnte. Aber da ging des nich. Aber dann hab ich die Zeit ausgenutzt, wenn mein Mann mittags geschlafen hat, dann hab ich mich auch einfach hingelegt, des hat mir kolossal geholfen. Oder wie gesagt, irgendwie 'ne ganz merkwürdige Beschäftigung vielleicht ... Knöpfe sortieren, Sachen plätten, die eigentlich gar nicht geplättet werden müssen, ja. Lenkt ab, bringt Bewegung. (Z. 582-611) 
Regelmäßige Entspannungsübungen helfen Frau M. zudem bei Rückenbeschwerden. Sie trifft dabei Vorsorge, dass sie nicht von ihrem Mann gestört wird, sodass sie ganz abschalten kann.

Frau M.: Also, wenn mein Rücken da mal gestreikt hat, da hab ich immer so'n paar Übungen gemacht: Lang auf den Boden legen, die Knie so anziehn und hin und her gaukeln. Das massiert so'n bisschen den Rücken, wenn ich mal wirklich nicht mehr konnte. Und dann die Arme nach hinten und so durchdehnen den ganzen Körper. Doch, des hab ich schon gemacht. Also, des kostet nicht mehr als fünf Minuten, die hatt ich denn auch. Also, sagen wa mal, die hab ich mir genommen, nich. Und wenn's zu der Zeit war, wo mein Mann abends schon mal geschlafen hat, nich. Nich, dass ich denn in den fünf Minunten durch „Ach, komm doch mal, jetzt ist hier irgendwas“, dann plötzlich wieder hoch musste dann is die Übung ja wieder hin. (Z. 295-303)

Auch das gelegentliche Hüten des Enkelkindes, damit die Tochter zum Sport gehen kann, sieht Frau M. nicht so sehr als Verpflichtung sondern eher als Möglichkeit ,aufzutanken“.

Frau M.: (...) Und also, des war so'ne Art Lebenselixier, diese Kleine, und isses noch. (...) da fahr ich dann hin und hüte die Kleine da, ja. Das HILFT einem, nich. (Z. 100-109)

Frau M. passt die Auslebung eigener Bedürfnisse (Pflege der eigenen Bedürfnisse) flexibel an die Anforderungen der Situation an. Zu Beginn der Erkrankung ihres Mannes hatte sie kaum Energie für andere Interessen. So beschränkt sie sich auf Freizeitaktivitäten, die wenig Aufwand bedeuten. Mit den Fortschritten ihres Mannes ist jedoch ihr Interesse an Vorträgen und Ausstellungen wieder geweckt, die sie vor der Erkrankung regelmäßig mit ihrem Sohn besucht hat.

Interviewerin: Und in der Zeit der Pflege, wo so die Aktivitäten 'n bisschen ausgefallen sind, die Sie normalerweise gemacht haben: Hat Ihnen das was ausgemacht?

Frau M.: (KLEINE PAUSE) Eigentlich wenig, eigentlich wenig. Ich war so konzentriert drauf, jeden - und wenn's noch so'n geringer Fortschritt war, den mein Mann machte - zu registrieren und mich damit zu befassen. Und denn da vielleicht auch drüber nachzudenken, was man vielleicht noch tun könnte. Nee, des hab ich eigentlich // eigentlich hab ich nüscht vermisst. Wenn wirklich Zeit war, na dann hab ich 'ne Kleinigkeit gelesen oder'n Kreuzworträtsel gemacht oder mir im Fernsehn 'ne Sendung angeguckt, die mich interessierte, nich. Ich 
bin früher öfter mal mit meinem Sohn $\mathrm{zu}$ Vorträgen gegangen und $\mathrm{zu}$ Ausstellungen. Des ist mir vielleicht'n bisschen schwer gefallen, des nun gänzlich fallen zu lassen. Jetzt sind wa so weit, dass wa sagen, wenn mal 'ne schöne Ausstellung ist, ähm, gucken wa uns die wieder an. (...) Das interessierte meinen Mann aber nie, der hat für Altertümer und sowat, naja, da hat er keinen Draht, der ist immer lieber gewandert in früheren Jahren. Und mein Sohn und ich haben uns dann sowas immer ausgesucht und sind da zur Ausstellung gegangen. (...) $\mathrm{Na}$, und da wollen wa wieder anschließen, wenn jetzt eine attraktive Ausstellung hier ist in Berlin, dann gehn wa hin. Mein Mann wird wohl nicht mitkommen, eben weil seine Interessen nich in Altertümern liegen (...) (Z. 396-423)

Auch zum Yoga-Kurs geht sie wieder, seitdem es ihrem Mann besser geht:

Frau M.: (...) Ich muss dazu sagen, ich habe auch immer Yoga jemacht, schon/schon 13 Jahre lang. Das war natürlich, als mein Mann jetzt so sehr krank war, weggefallen, aber das hab ich im Frühjahr wieder aufgegriffen und vielleicht hat mir des sehr geholfen. (Z. 279-282)

Sicherlich erleichtert ihr die Tatsache, dass sie früher schon Dinge ohne ihren Mann unternommen hat, die Pflege eigener Bedürfnisse. Sogar leichter Unmut ihres Mannes hält Frau M. nicht davon ab, Aktivitäten, die ihr wichtig sind, nachzugehen:

Interviewerin: Wie steht Ihr Angehöriger, Ihr Mann dazu, dass Sie zu dem Angehörigenkurs gegangen sind und jetzt auch noch zu der Gesprächsgruppe?

Frau M.: Na ja, ich hab den Kurs angefangen, da war er noch im Krankenhaus, und nachher tauchte des dann schon mal: Na musste denn dahin gehen? Und na ja, ich hab ihm das erklärt, wieso und warum und was ich dabei finde, wie ich des sehe: Na, denn is gut, dann geh mal. Er hat sich dann genau die Uhrzeiten sagen lassen und war dann zufrieden: Gott sei Dank, da biste ja wieder. (Z. 800806)

Die soziale Einbindung des Ehepaares M. ist gut, sodass Unterstützung durch das soziale Netzwerk gegeben ist. Frau M. nimmt dankbar Fahrdienste und Einladungen durch Freunde an (Inanspruchnahme sozialer Unterstützung):

Interviewerin: Gibt es noch andere Personen, die Sie auch um Hilfe bitten könnten?

Frau M.: Ja (ZÖGERLICH). Wir haben im Freundeskreis ... ich sage mal so, so in der Kegelgruppe so Freundschaften, doch. Also, und die Leute sind auch bemüht jewesen, meinen Mann im Krankenhaus zu besuchen. Zuerst war er ja nicht ansprechbar, also da mussten erst einige Wochen drüber hingehen, eh dann 
diese Freundschaften im Krankenhaus mal nach'm Rechten sehen konnten, nich. Ja und langjährige Bekanntschaften noch aus den Kindertagen unserer Kinder. Da sind so Freundschaften erhalten geblieben, die haben sich auch bemüht und die sind auch jetzt sehr hilfreich. Mein Mann kann ja nun keine großen Strecken laufen und die Leute, von denen ich jetzt spreche, die haben in der Nähe vom Flughafen Tegel 'n Garten. Wenn des Wetter einigermaßen is, war ja in diesem Sommer nich so doll, dann hamse uns schon abgeholt und wieder nach Haus jebracht, is natürlich wunderbar. (Z. 123-133)

Besonders in der Anfangszeit bat sie ihren Sohn häufiger um Fahrdienste ins Krankenhaus und, als ihr Mann wieder zuhause war, um „Pflegevertretung“, wenn sie selbst länger aus dem Haus war (z. B. den Angehörigenkurs besuchte).

Nicht nur instrumentelle Hilfe durch das soziale Umfeld, auch die emotionale Unterstützung haben für Frau M. eine entscheidende Bedeutung für die Bewältigung ihrer Situation. Neben ihrer unmittelbaren Familie gibt ihr auch eine Jugendfreundin, die zwar weiter weg lebt aber mit der sie regelmäßig telefoniert, emotionalen Beistand (Z. 468-476). Darüber hinaus erlebt Frau M. die Unterstützungsangebote im Rahmen des Angehörigenprojekts als sehr hilfreich:

Frau M.: (...) GANZ HILFREICH, muss ich dazu sagen, war hier die Frau H., ja. Also, des war so richtig, also wenn man so ein Anlehnungsbedürfnis hatte, konnte man ihr des bei der nächsten Stunde entweder, zuerst war des ja'n Kurs und dann die Gesprächsrunde, oder ich hab auch zwischendurch einfach mal bei ihr angerufen. Irgendwie, hach, irgendwie konnte man da was loswerden, was mit dieser Erkrankung meines Mannes zu tun hatte, und sie wusste 'ne Antwort drauf, man hatte nicht das Gefühl, man STÖRT irgendjemanden bei seiner eigenen Arbeit, bei seinen eigenen Gedankengängen, das war eigentlich UNWAHRSCHEINLICH hilfreich. Das hört sich vielleicht irgendwo ganz lächerlich an, isses aber nich. Das hat unwahrscheinlich geholfen. Ich war zuerst, muss ich sagen, skeptisch, als mir angeboten wurde: Wollnse da den Kurs machen? (...) und des war wunderbar, dass sich anschließend an diesem Kurs, diese Gesprächsrunde anschloss und jetzt nochmal is.(...) Das hat mir persönlich sehr geholfen, meinem Mann vielleicht im übertragenen Sinne auch. Aber diese Stunden hier bei Frau H. und auch mancher Anruf dann zwischendurch bei ihr hat mir persönlich sehr über die Runden geholfen, ja. (Z. 68-85)

Zeitweilig ließ sich Frau M., wie oben bereits erwähnt, auch durch einen häuslichen Pflegedienst unterstützen (Inanspruchnahme professioneller Unterstützung). Diese Hilfe war ihr so viel Wert, dass sie bereit war, die Leistungen selbst zu finanzieren, nachdem sie nicht mehr durch die Krankenkasse übernommen wurden: 
Frau M.:(...) Zuerst hatt ich auch noch - nach der Lungenentzündung - morgens immer 'ne Hauskrankenpflege und diese Frau, die da kam, die hat mir des denn auch alles noch besser zeigen können, als wenn ich mich da selber versucht hätte. Sie hatte die ersten Male meinen Mann gebadet. Sie hatte dann zwar immer nur einmal pro Woche dafür Zeit, aber det war wunderbar, diese Hilfestellung. Und dann hab ich's zwischendurch noch an einem anderen Wochentag probiert. Und nachdem die nun nicht mehr kommen konnte, von der Kasse kriegt ick des nicht mehr, des hab ich nur bis Ende Dezember gekriegt. Dann hat ich mir des noch zwei Monate selbst bezahlt, Januar und Februar noch, und dann hamwa des auslaufen lassen. Und dann hat es mein Mann auch selbst bewältigt. (Z. 209-218)

\section{Beziehungsqualität und Pflegemotivation}

Während sich bei Frau T. in der ersten Fallbeschreibung aus vielen ihrer Äußerungen ambivalente Gefühle gegenüber ihrem Mann und Spannungen innerhalb ihrer Beziehung herauslesen ließen, ist die Beziehung der Eheleute M. relativ spannungsfrei und geprägt von Offenheit. Frau T. benennt ihre Belastung nur indirekt, gleichwohl sie, gemessen an den quantitativen Daten, recht hoch ist. Kennzeichnend für Frau M. ist dagegen, dass sie sich sehr reflektiert mit ihren eigenen Emotionen auseinandersetzt und Gefühle der Überforderung oder des Angebundenseins nicht negiert. So spricht sie in der folgenden Textpassage offen das Gefühl der Einschränkung durch die unterbrochene Nachtruhe an:

Frau M.: (...) erst hatte er 'ne Ente, aber des ist so 'ne Sache, die war ihm dann nich aktiv genug. Also geht man dann doch zur Toilette, nich. Auch gar nich mal der Weg jetzt, vom Bett zur Toilette, war so schlimm. Dieses ständige aus dem Schlaf gerissen $\mathrm{zu}$ werden. Man hatte ja denn die Ohren schon so eingestellt, wenn sich da was muckst // er schlief ja zu der Zeit im anderen Zimmer, im anderen Bett (...) wo er einfacher reinkam und besser aufstehen konnte. Also, das hab ich als schlimm empfunden, dass ich dauernd gestört werde in meiner eigenen Ruhe, die ich so DRINGEND gebraucht hätte. Er musste - muss ich vielleicht noch dazu sagen - vor der Schilddrüsenoperation bis zu drei Liter trinken pro Tag und dann könnense sich vorstellen, wie oft so'n Mensch dann Wasser lassen muss. Des kam dann natürlich hinzu, deshalb musste er ja auch drei-, vier-, manchmal, wenn ich Pech hatte, auch fünfmal inner Nacht aufstehn. Und des is natürlich etwas, diese Kraft kann man kaum wieder hinkriegen, nich. Ich meine, jetzt - denk ich mal - bin ich eigentlich wieder so hergestellt, dass ich des überwunden habe. Aber in der ersten Zeit, als es MEINEM MANN besser ging, da fing ich plötzlich an, Probleme zu entwickeln: Mir tat alles weh, vom Kopf bis zu den Zehen. Bin dann zur Ärztin gegangen (...) Sagte sie zu mir: Das ist der Nachlauf, des sind ihre Nerven, die ihnen da jetzt Streiche spielen. (Z. 250-271) 
Anders als Frau T. identifiziert sich Frau M. nicht übermäßig mit der Pflegerolle und behandelt ihren Mann nach wie vor als gleichwertigen Partner. Dies zeigt sich auch darin, dass sich Frau M. nicht ausschließlich als psychische Stütze und Pflegende ihres Mannes empfindet, sondern auch durch ihn emotionalen Halt erfährt, wie sie in der folgenden Äußerung beschreibt:

Interviewerin: Gibt es in der derzeitigen Situation etwas, was Ihnen Trost gibt oder Ihnen hilft?

Frau M.: (...) Na ja, und dann immer wieder meine Familie, ja ... und selbst mein Mann, trotz seiner Krankheit, ja. (Z. 649-655)

Indem sie ihren Mann die eigene Belastung und Traurigkeit sehen lässt und ihm erlaubt, ihr emotionale Unterstützung zu bieten, wenn sie sie braucht, reduziert sie ihn nicht ausschließlich auf die Rolle des Kranken und Hilfsbedürftigen.

Die eigenen Interessen, denen Frau M. schon immer in ihrer Beziehung nachgegangen ist, pflegt sie noch trotz der Erkrankung ihres Mannes, wenn auch in verminderter Form. So geht sie weiterhin ihrem Yoga-Sport nach und besucht hin und wieder Ausstellungen. Die Beziehung war vor dem Schlaganfall von Gleichberechtigung und gegenseitiger Akzeptanz von Freiräumen geprägt und hat sich in dieser Hinsicht nicht verändert.

Die Unterstützung ihres Mannes geht für Frau M. mit keinerlei Zweifeln einher, sie empfindet regelrecht Dankbarkeit dafür, dass ihr die Kraft und die Fähigkeiten gegeben sind, diese Aufgabe leisten zu können.

Interviewerin: Aber für Sie war es selbstverständlich, dass Sie die Pflege ihres Angehörigen übernehmen?

Frau M.: Ja - ohne Wenn und Aber.

Interviewerin: Und gab es jetzt auch von außen die Erwartungshaltung, dass Sie die Pflege übernehmen sollen?

Frau M.: (PAUSE) Ja, ich weiß das jetzt nicht ganz einzuordnen, wie Sie meinen „von außen“...

Interviewerin: Also, ja zum Beispiel auch von den Kindern oder von anderen Bekannten ...

Frau M.: Ja so, schön, wahrscheinlich haben alle vorausgesetzt, dass ich des 
mache. (...) Und ich bin DANKBAR, dass ich's konnte. Wenn ich jetzt ausgefallen wäre ... na schön, dann hätte noch der Pflegedienst vielleicht erweitert werden können, wenn ich ausgefallen wäre. Aber ein Mensch in der Situation kann sich einfach selbst nicht behelfen und ich bedaure jeden Menschen, der vielleicht auf ein oder zwei Stunden am Tag auf so'n Pflegedienst angewiesen ist. Ich stelle mir det ganz, ganz schrecklich und katastrophal vor. Jetzt vielleicht gar nicht mal hier von der körperlichen Pflege, aber so von der Ansprache. Es ist dann niemand, den man mal eben rufen kann, ja. Also des muss schrecklich sein. (...) Also, ich hab da auch keinen Gedanken verschwendet, als mein Mann an dem Morgen da im Bad zusammenklappte. Also, ich war natürlich so entsetzt, dass ich zuerst gar nicht begriffen hatte, was das eigentlich bedeutet. Aber ich hätte da gar nicht anders gekonnt, als mich um ihn zu kümmern, und ich muss - vielleicht riecht das jetzt so'n bisschen nach Eigenlob - aber ick bin auch jeden Tag im Krankenhaus gewesen, von Anfang an. (Z. 333-356)

\section{Interaktionen zwischen Bewältigungsverhalten und Intervention}

Frau M. verfügt bereits vor der Kursteilnahme über ein breites Repertoire an Bewältigungsstrategien. Dabei stehen die patientenzentrierten und ichzentrierten Strategien in einem ausgewogenen Verhältnis zueinander. Frau M. ist außerdem in der Lage, ihr Verhalten an die Erfordernisse der Situation anzupassen. Mit zunehmenden Fortschritten ihres Mannes in der Rehabilitation nimmt sie ihre Hilfeleistungen zurück und fördert seine Selbständigkeit. Ist die Pflege eigener Bedürfnisse zu Beginn der Erkrankung etwas in den Hintergrund getreten - gleichwohl nie ganz aufgegeben worden - räumt Frau M. ihnen im Verlauf des Rehabilitationsprozesses zunehmend mehr Platz ein.

Die offensive und aktive Form der Auseinandersetzung mit der Erkrankung ihres Mannes ist beste Voraussetzung dafür, Frau M. für die Teilnahme am Angehörigenkurs zu gewinnen. Zentrale Motivation für den Kursbesuch ist es, weitere Kenntnisse und Sicherheit im Umgang mit der Situation zu erlangen, wie Frau M. im folgenden Gesprächsausschnitt schildert:

Interviewerin: Sie haben von sich selbst aus auch schon den Angehörigenkurs angesprochen. Wissen Sie noch, was Sie motiviert hatte, am Angehörigenkurs teilzunehmen?

Frau M.: Ja. Ja also, des kann ich fast genau sagen: Hilfestellung ganz einfach. Aber gar nich jetzt mal im übertragenen Sinne, sondern bei den ganz praktischen 
Dingen, ja. Also, ich hab gedacht, des kann auf gar keinen Fall verkehrt sein: Du weißt gar nicht, wie's weiter geht, du musst damit umgehn können. Und ich hab ja denn auch gemerkt, es wurde alles angesprochen, wie setz ich meinen Angehörigen vom Bett in den Stuhl/in den Rollstuhl und umgekehrt. Wie kann ich ihm beim Waschen behilflich sein, wie kann ich behilflich sein, wenn er sich nicht anziehn kann. Ganz abgesehen davon, dass ja auch andere Dinge zur Sprache kamen, wie zum Beispiel mit dieser Aphasie, wie des // und dass diese Menschen ja sehr empfindlich sind durch ihre Krankheit. Des is bei meinem Mann wahrscheinlich immer noch der Fall, dass er dann anfängt zu weinen, dass er doch eben merkt, dass er doch 'ne Einschränkung hat. Es ist ja richtig, die ist ja da, aber mit dem, was jetzt an Einschränkungen ist, kann man leben. Aus dem Grunde hab ich den Kurs gemacht und hab alles nach Möglichkeit in mich aufgesaugt, dass ich im Zweifelsfalle immer darauf zurückkommen kann, ja. (...) (Z. 668-682)

In den Textbeispielen im Rahmen der vertiefenden Fallanalyse wurde deutlich, dass Frau M. vieles von dem im Kurs und durch Hospitationen erworbenen Wissen im Pflegealltag umzusetzen vermochte. Dazu gehören beispielsweise die ständige und korrekte Einbeziehung der vom Schlaganfall betroffenen Körperhälfte, die Anschaffung von Hilfsmitteln sowie der adäquate Umgang mit den neuropsychologischen Störungen und mit der Sprachstörung ihres Mannes.

Neben dem Erwerb rein praktischer Kenntnisse im Kurs ist Frau M. der Aspekt der emotionalen Unterstützung durch die Kurteilnehmer jedoch ebenso wichtig:

Frau M.: (...) Und nachher diese Stunden, die dann anschließend waren, des sind noch Stunden für die Angehörigen so zum Sich-hängenlassen-können, ja. Man nimmt sich ja so zusammen, man ist ja so konzentriert. Also, der Angehörige darf nich merken, wenn's einem mal nicht so richtig wohl zumute ist. Dann kann ich ihm nicht die Kraft geben, die er braucht. Aber wenn man hier dann drüber spricht: Also ich bin heute völlig k.o., das war so anstrengend oder so. Ist vielleicht alles auch ganz einfach, aber es sind hier alles Leute in ähnlichen oder gleichen Situationen. Sie können ja jetzt nich einem, der völlig fit ist, nu erzählen: Mir ist heute so, ich bin so kaputt und schlapp, weil ich das und das gemacht habe. „Ach ja?“, sagt der dann. Hier sagen die Leute: Kann ich Ihnen nachempfinden, ich hatte gestern det Problem. Ich weiß jetzt nicht, ob ich mich verständlich ausdrücken kann, was da so für Unterschiede bestehen $\mathrm{zu}$ jemandem, der nicht mit so'ner Situation konfrontiert ist, nich. Also, ich kann's nur jedem empfehlen und es wäre wünschenswert, wenn solche Kurse weiter fortgeführt werden. (Z. 687-699) 
Die emotionale Entlastung, die sie durch die Kursteilnahme erfährt, empfindet sie als so hilfreich, dass sie noch weiter an der Gesprächsgruppe teilnimmt.

Interviewerin: Was hat Sie bewogen, anschließend an der Gesprächsgruppe noch teilzunehmen?

Frau M.: (...) und auch der Werdegang, wie des woanders weiter gegangen ist // irgendwann verliert man sich ja sonst auch aus den Augen (...)// also mir hat es jedenfalls auch irgendwo 'nen Halt gegeben - dass ick mit der Misere nich alleine dastehe. (Z. 762-765)

Vergleicht man die beiden dargestellten Fälle miteinander, so fällt auf, dass bei Frau M. schon im Vorfeld günstige Voraussetzungen für eine funktionale Bewältigung der Erkrankung ihres Mannes bestehen: Die Kursteilnahme beginnt 2 Monate nach dem Akutereignis, während bei Frau T. der Schlaganfall ihres Mannes bereits 40 Monate zurückliegt. Die Teilnahme am Kurs kann bei Frau M. somit eher einer dysfunktionalen Bewältigung vorbeugen. Zudem ist das soziale Netzwerk des Ehepaars M. größer und tragfähiger als das der Familie T. Durch ihren biographischen Hintergrund und die Beziehungsqualität ist bei Frau T. außerdem eher als bei Frau M. eine Prädisposition gegeben, patientenzentrierte Bewältigungsstrategien an den Tag zu legen. Diese Faktoren tragen sicherlich dazu bei, dass bei Frau M. im Gegensatz zu Frau T. eine Abnahme der Belastungswerte im zeitlichen Verlauf zu verzeichnen ist, während bei Frau T. lediglich erste Veränderungen auf der Verhaltensebene zu beobachten sind. 


\section{Diskussion}

Die zentrale Frage der vorliegenden Arbeit betraf die Wirksamkeit eines Kurses für Angehörige von Schlaganfallpatienten. Eine Zielsetzung war es zu ermitteln, ob die Teilnahme am Kurs Auswirkungen auf die emotionale und körperliche Befindlichkeit der pflegenden Angehörigen hatte. Es wurde dabei sowohl eine quantitative als auch qualitative Methodik verwendet. Im Rahmen der quantitativen Untersuchung wurden die pflegebedingte Belastung, die emotionale und körperliche Befindlichkeit, der Informiertheitsgrad in Bezug auf Schlaganfallbezogenes Wissen sowie andere Parameter untersucht. Im qualitativen Untersuchungsteil wurden anhand von zwei vertiefenden Fallstudien Bewältigungsmechanismen differenziert untersucht. Des Weiteren wurde analysiert, inwiefern die Coping-Mechanismen Auswirkungen hatten auf den Nutzen, den die jeweiligen Angehörigen aus dem Kursbesuch ziehen konnten.

Im Folgenden werden zunächst die wesentlichen Ergebnisse aus dem quantitativen und dem qualitativen Untersuchungsteil diskutiert. Es folgen eine kritische Würdigung der Untersuchungsmethodik sowie eine Reflexion der methodischen Grenzen der Untersuchung. Anschließend werden Implikationen für die Caregivingund Interventionsforschung erörtert. Des Weiteren werden Folgerungen, die sich aus der vorliegenden Arbeit für die angewandte Angehörigenarbeit ergeben, diskutiert.

\subsection{Quantitative Befunde}

Ein zentrales Ergebnis der quantitativen Untersuchung war, dass die Kursteilnehmer eine signifikante Zunahme des Schlaganfall-spezifischen Wissens im Vergleich zur Kontrollgruppe zeigten. Weiterhin war der Anteil der pflegenden Angehörigen, die Entlastungsangebote über den gesamten Untersuchungszeitraum hinweg nutzten, in der Interventionsgruppe signifikant höher als in der Kontrollgruppe. Dieses Ergebnis steht im Gegensatz zu den Befunden von Toseland und Smith (1990). Die Autoren konnten bei pflegenden Angehörigen keinen positiven Effekt individueller Beratung auf die Nutzung (psycho-)sozialer Entlastungsangebote nachweisen. Hier zeigt sich der Vorteil eines Kurses im Vergleich zu Einzelberatungen: Berichte positiver Erfahrungen von einzelnen Kursteilnehmern können andere Pflegepersonen motivieren, 
Entlastungsangebote zu nutzen. Offensichtlich war der Kurs somit erfolgreich, die Einstellungen der Angehörigen gegenüber der Nutzung psychosozialer Entlastungsangebote positiv zu beeinflussen. Dies ist ein ermutigendes Ergebnis, wenn man bedenkt, dass Angehörige von Pflegebedürftigen mit kognitiven Beeinträchtigungen Unterstützungsangebote seltener und kürzer nutzen, als pflegende Angehörige von Menschen anderer Erkrankungsgruppen (Pedlar \& Biegel, 1999).

Ein weiteres positives Ergebnis war, dass die subjektiv wahrgenommene Belastung bei den Kursteilnehmern in dieser Studie abnahm, während sie für die Mitglieder der Kontrollgruppe konstant blieb. In die gleiche Richtung weisen die Ergebnisse aus dem Fragebogen zum subjektiv erlebten Nutzen des Kurses, in dem durchgängig die Mehrheit der Angehörigen angab, dass der Kurs hinsichtlich diverser Aspekte eine positive Wirkung hatte.

Betrachtet man jedoch die Ergebnisse für die Variablen Depression, Angst und körperliche Beschwerden, könnte man $\mathrm{zu}$ dem Schluss gelangen, dass Angehörigenkurse - in der hier angebotenen Form - nicht effektiv sind. Dieses Dilemma zeigt sich auch in anderen Studien (Cooke, McNally, Mulligan, Harrison \& Newman, 2001; Pusey \& Richards, 2001; Thompson \& Briggs, 2000; Zarit, Gaugler \& Jarrott, 1999): In den meisten Untersuchungen äußern sich die Teilnehmer der Angehörigenkurse sehr positiv und berichten von verschiedenen gewinnbringenden Aspekten, die aus der Teilnahme resultieren. Betrachtet man jedoch allein die standardisierten Instrumente, die Gruppenunterschiede nachweisen sollen, zeigen sich kaum Unterschiede. In nur einem Drittel der insgesamt 40 Studien, die in dem Überblicksartikel von Cooke et al. (2001) analysiert wurden, ließ sich nachweisen, dass Interventionen das psychische Wohlbefinden der Pflegenden fördern, soziale Faktoren verbessern oder das Belastungserleben verringern. Die Verbesserung des Krankheitsbezogenen Wissens als outcome-Variable wurde erst in den neunziger Jahren häufiger in Interventionsstudien verwendet. Von den 16 Studien, die im Überblicksartikel von Cooke et al. (2001) untersucht wurden, zeigten 11 (69\%) Verbesserungen in dieser Variable. Von diesen 11 Studien konnten in nur 3 Arbeiten außerdem Verbesserungen des psychischen Wohlbefindens nachgewiesen werden. 
Daraus könnte man die recht fatalistische Schlussfolgerung ziehen, dass es wenig Möglichkeiten gibt um die Belastung Pflegender zu reduzieren. Dieses Dilemma könnte jedoch auch zu einer Neubewertung führen, ob die bisherigen Methoden Interventionserfolg $\mathrm{zu}$ messen, tatsächlich angemessen sind (Bond, 2000; Moriarty, 1999). Möglicherweise bildet die Auswahl der Messinstrumente in bisherigen quantitativen Studien die Nuancen der Pflege nicht adäquat ab und es empfiehlt sich eine Orientierung an Modellen, die die sehr komplexe Natur der Pflege besser einfangen. In diesem Zusammenhang argumentieren Lawton und Mitarbeiter, dass die Auswahl der Messinstrumente dem multifaktoriellen Charakter von Belastung in Pflegebeziehungen Rechnung tragen sollte (Lawton, Kleban, Moss, Rovine \& Glicksman, 1989). Unter Umständen wäre es sinnvoller, spezifische Verhaltensänderungen zu messen als globale psychologische Veränderungen. In der vorliegenden Studie waren Veränderungen vor allem auf der Verhaltensebene festzustellen. Dies war im Rahmen des quantitativen Erhebungsteils vor allem durch eine verstärkte Nutzung von Entlastungsangeboten sowie bei den Angaben der Angehörigen zum subjektiven Nutzen (Kapitel 6.5) deutlich geworden. In der qualitativen Untersuchung waren bei der funktional bewältigenden Frau M. viele positive Verhaltensänderungen nach Teilnahme am Kurs zu verzeichnen. Auch bei der eher dysfunktional bewältigenden Frau T. (siehe Kapitel 8.3.1) waren erste Ansätze einer Verhaltensänderung ersichtlich. Für künftige Interventionsstudien wäre daher zu empfehlen, Indikatoren auszuwählen, die stärker auf eine Verhaltenskomponente, möglicherweise auch auf die Interaktion zwischen Pflegebedürftigem und Pflegenden abzielen.

Diese Überlegung wird durch einen Befund von Haley, Brown und Levine (1987) gestützt. Die Autoren stellten fest, dass für viele Pflegende eher im Vordergrund steht, ihre Effizienz als Pflegeperson zu verbessern, als das Ausmaß ihrer Belastung zu verringern. Außerdem darf nicht vergessen werden, dass es sich in der vorliegenden Studie überwiegend um chronische Pflegesituationen handelte, die zum Teil schon über Jahre hinweg bestanden. Emotionale Verarbeitungsmuster haben sich in dieser Zeit möglicherweise verfestigt und sind relativ resistent gegenüber Veränderungen (Jang, Clay, Roth, Haley \& Mittelman, 2004; Zarit, Todd \& Zarit, 1986). Denkbar wäre, dass 
eine Abnahme psychischer und psychosomatischer Symptome, wie sie durch standardisierte psychopathologische Skalen erfasst werden, erst festzustellen ist, wenn über einen längeren Zeitraum hinweg Entlastungsangebote genutzt wurden.

Ein weiterer kritischer Punkt der vorliegenden Studie ist in der Auswahl der Gruppen zu sehen. Aus ethischen und praktischen Überlegungen konnte keine Randomisierung der Teilnehmer stattfinden, was zur Folge hatte, dass sich die Gruppen a priori in systematischer Weise unterschieden. Das Ausmaß der Pflegebedürftigkeit, gemessen anhand des Barthel-Index, war in der Treatmentgruppe signifikant höher als in der Kontrollgruppe. Dies konnte allerdings durch die Parallelisierung der Gruppen ausgeglichen werden (siehe Kapitel 5.2), was jedoch eine Reduktion der Stichprobengröße zur Folge hatte. Dem kann jedoch positiv gegenüber gestellt werden, dass bei diesem methodischen Vorgehen der Stichprobenauswahl eine hohe ökologische Validität zuzusprechen ist, da in der Realität auch nur eine begrenzte Anzahl an Angehörigen derartige Angebote in Anspruch nehmen (Fredman et al., 2004).

Es wäre sicher hilfreich gewesen, Faktoren systematisch zu erheben, die zur Nicht-Teilnahme am Angehörigenkurs führten. So hätte ein besseres Bild darüber gewonnen werden können, welche Personengruppe aus welchen Gründen nicht mit dem Interventionsangebot erreicht wird. Dies war jedoch aus forschungsökonomischen Gesichtspunkten nicht möglich.

Auch der Zeitpunkt der Intervention ist ein wichtiger Faktor. Der effektivste Zeitpunkt, eine Intervention anzubieten, ist ein Zeitpunkt, der besonders sensitiv für Veränderungen ist. Der optimale Zeitpunkt für eine Intervention variiert nach Stadium, Art und Schwere der Erkrankung (Zarit \& Leitsch, 2001). Dadurch bedingt, dass sich die Pflegebedürftigen der teilnehmenden Angehörigen in einer Tagesklinik befanden und zuvor meist mehrere Wochen in einer stationären Rehabilitationseinrichtung zugebracht hatten, waren im Median seit Beginn der Erkrankung 9 Monate vergangen. Da Patienten zum Teil aufgrund eines ungenügenden ambulanten Therapieangebots wiederholt teilstationär aufgenommen werden, lag das Akutereignis in einigen Fällen (so wie bei dem Ehemann von Frau T., deren Fall in Kapitel 8.3.1 ausführlich analysiert wird) sogar Jahre zurück. Es ist also davon auszugehen, dass die Bewältigungsmuster schon relativ chronifiziert waren und sich Belastungen über die Zeit aufsummiert hatten. 
Idealerweise sollten Angehörige noch während der stationären Rehabilitation, also relativ früh im Krankheitsprozess, die Möglichkeit erhalten, an einem Kurs teilzunehmen. Möglicherweise ließe sich damit verhindern, dass sich überhaupt dysfunktionale Bewältigungsstrategien herausbilden.

Ein weiterer Aspekt betrifft den Zeitrahmen der Intervention. Die Dauer der hier untersuchten Gruppenintervention betrug 10 Sitzungen. Es ist zu hinterfragen, ob dieser Zeitrahmen ausreichend bemessen war. Möglicherweise war der Zeitraum zu kurz, um deutliche Effekte zu produzieren. In Bezug auf die Effektivität von Tagespflege zeigte sich zum Beispiel, dass ihre Nutzung erst dann einen Entlastungseffekt bringt, wenn sie mindestens 8 Stunden pro Woche umfasst (Knight et al., 1993). In ähnlicher Weise wäre es im Falle einer psychoedukativen Intervention denkbar, dass ein größerer Zeitrahmen notwendig ist, um Veränderungen im Wohlbefinden Angehöriger zu erzielen.

Ebenso war möglicherweise der Zeitrahmen der Datenerhebung zu eng gewählt. Bei den Teilnehmern, die durch den Kurs motiviert wurden, psychosoziale Dienste in Anspruch zu nehmen oder andere Aspekte des Pflegealltags zu verändern, verringert sich möglicherweise erst nach einem größeren Zeitraum die psychische Belastung. Eine Langzeitstudie über einen Zeitraum von etwa 2 bis 3 Jahren ist naturgemäß aufwändig und konnte im Rahmen der vorliegenden Studie nicht verwirklicht werden. Aber da Krankheitsverarbeitung und -bewältigung langwierige Prozesse sind, entspräche eine solche eher den Anforderungen des Untersuchungsgegenstandes.

Die Ergebnisse der quantitativen Studie implizieren, dass vor allem Angehörige, die sich relativ wenig informiert fühlen, das Angebot eines Angehörigenkurses nutzen. Tendenziell sind außerdem Angehörige, die insgesamt ein höheres Belastungsniveau aufweisen, eher an einer Kursteilnahme interessiert. Dies spiegelt sich in dem recht hohen Belastungsniveau bei den Angehörigen in der Interventionsgruppe wieder. Somit konnten die Befunde einer Studie von Evans und Baldwin (1989) nicht bestätigt werden. Diese Autoren fanden, dass Angehörige, die selbst ein hohes Ausmaß an depressiven Symptomen vorwiesen oder die einen kognitiv und motorisch schwer beeinträchtigten Patienten versorgten, Angebote für Angehörige eher nicht 
wahrnehmen.

Ein weiterer Diskussionspunkt betrifft die Frage, ob die inhaltliche Konzeption der Intervention mit den Bedürfnissen der Teilnehmer übereinstimmte. Differenzierte Bedarfsanalysen für eine angemessene Unterstützung speziell von Angehörigen von Schlaganfallpatienten liegen bisher nur in Ansätzen vor (Baskett, Broad, Reekie, Hocking \& Green, 1999; Quine, David \& Pierce, 1993; Rosenthal, Pituch, Greninger \& Metress, 1993). Übereinstimmend wird für die Phase der stationären Rehabilitation der Patienten der hohe Informationsbedarf von Angehörigen betont und inhaltlich unterlegt (z. B. Aufklärung über die Erkrankung, Ernährungsberatung, Inkontinenzberatung, Aufklärung über spezielle Symptome wie Sprachstörungen oder neuropsychologische Störungen). Es besteht jedoch Forschungsbedarf $\mathrm{zu}$ der Frage, welche dieser Informationen zu welchem Zeitpunkt den individuellen Bedürfnissen der Angehörigen gerecht werden und in welcher Form sie vermittelt werden sollten (Gründel, Lehmann, von Cramon \& Wilz, 2001).

Ein häufiges Problem von Interventionsstudien im Bereich der familiären Pflege sind Boden- und Deckeneffekte von Tests oder Fragebögen. So zeigt in CaregivingStudien eine substanzielle Anzahl von Angehörigen, oft ein Drittel oder mehr, typischerweise nur eine geringe Ausprägung depressiver Symptome (Cooke et al., 2001; Zarit \& Leitsch, 2001). Diese Individuen können also kaum eine Verbesserung zeigen, da ihre Skalenwerte bereits am unteren Skalenende liegen. Somit ist die Wahrscheinlichkeit, Veränderungen zu entdecken, reduziert.

Dieses Problem hängt damit zusammen, wie Stichproben typischerweise in der Angehörigenforschung gewonnen werden. Personen werden in eine Interventionsstudie aufgenommen, weil sie bestimmte Kriterien in Bezug auf familiäre Pflege erfüllen. Es wird vorausgesetzt, dass diese Angehörigen ein erhöhtes Stresserleben haben, was jedoch nicht der Fall sein muss. Immerhin zeigten in der vorliegenden Stichprobe 20 Prozent der Angehörigen niedrige Werte (mehr als eine Standardabweichung unterhalb des Mittelwertes) sowohl in der Depressionsskala als auch im Angstfragebogen und 21 Prozent wiesen bei körperlichen Beschwerden niedrige Werte auf. Im Vergleich zu Interventionsstudien im Bereich der Psychotherapieforschung, wo Teilnehmer nur ausgewählt werden, wenn sie eine spezifische Ausprägung an psychischen Symptomen zeigen, ist das Auswahlkriterium in der Angehörigenforschung meist, wie auch in der 
vorliegenden Studie, die Pflegetätigkeit. Dieses Problem könnte umgangen werden, indem als zusätzliches Auswahlkriterium ein gewisses Maß an Belastung oder an Psychopathologie, erhoben anhand von standardisierten Messinstrumenten, festgelegt würde.

\subsection{Qualitative Befunde}

Bereits in der Diskussion der quantitativen Befunde wurde darauf eingegangen, dass für eine sinnvolle Interpretation der Interventionsergebnisse die Bewältigungsmechanismen der Angehörigen stärker einbezogen werden müssen. Die im Rahmen der qualitativen Studie erstellten Fallanalysen leisteten einen entscheidenden Beitrag zum besseren Verständnis der Prozesse, die in der „Blackbox“ zwischen Interventionsmaßnahme und erhobenen Outcome-Maßen wirksam waren.

\section{Repertoire an Bewältigungsmechanismen}

Bei der Analyse der Bewältigungsmechanismen wurde die Einteilung nach Lämmler (1998) in patientenzentrierte und ichzentrierte Strategien zugrunde gelegt. Die Klassifikation erwies sich auch für das Datenmaterial in dieser Untersuchung als sinnvoll. Diese Taxonomie diente Lämmler als Hintergrundfolie, um pflegende Angehörige als funktionale oder dysfunktionale Bewältigerinnen zu typisieren. Auch in der vorliegenden Studie konnte dieser Typisierung gefolgt werden. Erfolgreiche Bewältigerinnen zeichnen sich dadurch aus, dass sie eigene Bedürfnisse zeitweilig zurückstellen können, wenn es die Bedingungen verlangen, sich aber nicht ausschließlich mit der Pflegerolle identifizieren. Sie sind in der Lage, das eigene Verhalten an die Fähigkeiten des Patienten anzupassen. Das bedeutet, dass sie soviel Hilfe wie nötig und so wenig wie möglich geben. Nicht-erfolgreiche Bewältigerinnen vermindern im Gegensatz dazu nicht ihr pflegerisches Engagement, auch wenn Fortschritte in der Rehabilitation zu verzeichnen sind. Die Selbständigkeit des Patienten wird nur unzureichend gefördert und Kompetenzen des Patienten werden nicht entsprechend gewürdigt. Bei diesen Angehörigen wird die Pflegetätigkeit zum zentralen Lebensinhalt. Eigene Bedürfnisse werden negiert. Dennoch ziehen dysfunktionale Bewältigerinnen einen psychischen Gewinn aus ihrem Verhalten. Durch die Überidentifikation mit der Pflege gelingt es ihnen, Ängste, Unsicherheiten und 
Schuldgefühle zu reduzieren.

Auch Lämmler (1998) fand in seiner Studie, dass Schuldgefühle in besonderem Maße zu einem dysfunktionalen Coping prädisponieren. Er argumentierte, dass diese durch eine übertriebene Pflege bis hin zur Selbstaufgabe kontrolliert werden, dies erfüllt in einigen Fällen gleichzeitig die Funktion einer Selbstbestrafung. Oft mündet das Bemühen, vorhandene Ängste und Schuldgefühle mit einer aufopfernden Pflege zu reduzieren, jedoch in einem Teufelskreis: Verausgaben sich Angehörige bei der Pflege völlig, führt dies zwangsläufig zu ambivalenten Gefühlen gegenüber dem Pflegebedürftigen. Diese Ambivalenz verstärkt wiederum die Schuldgefühle, so dass der pflegerische Einsatz möglicherweise noch weiter gesteigert werden muss, um die Schuldgefühle wieder zu reduzieren. Lämmler betont, dass dysfunktionale Bewältigerinnen besonders anfällig sind für Selbstverleugnungen und die Ausblendung der eigenen Bedürfnisse.

Betrachtet man genauer das Repertoire an Bewältigungsstrategien, das die Angehörigen in der vorliegenden Untersuchung an den Tag legten, zeigt sich ein Unterschied in der Bandbreite. Von den elf Angehörigen, mit denen ausführliche Interviews durchgeführt wurden, wurden sieben nach der Klassifikation von Lämmler (1998) als funktionale Bewältigerinnen und vier als dysfunktionale Bewältigerinnen eingestuft. Insgesamt zeigten die sieben funktional bewältigenden Angehörigen eine größere Bandbreite an unterschiedlichen Copingstrategien (minimal 13, maximal 22 Strategien insgesamt). Dagegen lag die Bandbreite der Strategien bei den vier als dysfunktional bewältigend klassifizierten Angehörigen zwischen 9 und 11. Ein ähnliches Muster fanden auch Nolan, Ingram und Watson (2002) in ihrer qualitativen Studie mit Angehörigen von Demenzerkrankten.

Es gibt Hinweise in der Literatur (vgl. Lämmler 1998), dass Angehörigen mit dysfunktionalem Bewältigungsverhalten von vornherein weniger Coping-Ressourcen zur Verfügung stehen. Daraus ließe sich also ein höheres Vulnerabilitätsrisiko ableiten. Bewältigungsversuche mit ungünstigem Ergebnis dürften das Bewältigungsvermögen noch weiter herabsetzen. Dass damit aber die Effektivität zukünftigen Copingverhaltens gewissermaßen schon vorhergesagt werden kann, kann in dieser deterministischen Form nicht bestätigt werden. Auch bei Angehörigen mit insgesamt dysfunktionalem 
Bewältigungsverhalten sind Ansätze von Verhaltensänderung zu beobachten, die längerfristig durchaus in ein funktionales Coping übergehen könnten. Die Klassifikation einer pflegenden Angehörigen als Person mit funktionalem oder dysfunktionalem Bewältigungsverhalten bleibt daher immer eine vorläufige, die sich in der weiteren Auseinandersetzung mit dem Schlaganfall ändern kann (siehe Lämmler, 1998).

Wilz (2002) verwendet in ihrer qualitativen Studie mit Angehörigen Demenzkranker eine andere Begrifflichkeit, sie arbeitet mit den Begriffen emotionale Abhängigkeit versus emotionale Autonomie, inhaltlich stimmt die Taxonomie mit der Lämmlers jedoch im Großen und Ganzen überein. Wilz fand, dass sich eine starke emotionale Abhängigkeit des Pflegenden von der erkrankten Person negativ auf die Anpassung an den Krankheitsprozess und den Umgang des Angehörigen mit den krankheitsbedingten Einschränkungen auswirkt. Eine erfolgreiche Bewältigung geht hingegen mit einer hohen emotionalen Autonomie einher. Aus theoretischer Sicht wird argumentiert, dass die erfolgreiche Bewältigung der Pflegesituation ein hohes Ausmaß an emotionaler Unabhängigkeit von den Angehörigen erfordert, um etwa mit schwierigen Verhaltensweisen der Kranken (z. B. Aggressivität) adäquat umgehen zu können, ohne Schuldgefühle zu erleben. Als Merkmale für „emotional abhängige Beziehungen“" hebt Wilz in diesem Kontext folgende Aspekte hervor:

- dem Pflegebedürftigen keine Grenzen setzen können;

- ein ständiges Zurückstellen eigener Bedürfnisse;

- das Erleben der eigenen Unentbehrlichkeit in der Pflege;

- Schuldgefühle bezüglich eigener Aktivitäten, wenn man sich zeitweise nicht um den Kranken kümmert;

- Unfähigkeit zur Annahme von Hilfe;

- Verknüpfen des eigenen Wohlbefindens mit dem des Kranken;

- Verleugnen oder unrealistische Interpretation objektiv vorhandener Krankheitssymptome;

- das Fehlen von eigenen Zukunftsperspektiven außerhalb der Pflegesituation.

Wilz betont, dass im zeitlichen Verlauf erhebliche Veränderungen im Bewältigungsverhalten und in der Belastungsverarbeitung auftreten können, in der 
Regel stellen sich diese allerdings nur über einen langen Zeitraum ein. Als zentrale Faktoren, die eine Veränderung des Copingverhaltens und des Belastungserlebens sowohl in positive als auch in negative Richtung bewirken, führt sie folgende Aspekte an: Veränderungen der Krankheitssymptomatik (z. B. Zunahme von Verhaltensauffälligkeiten), Teilnahme an einer therapeutisch angeleiteten Angehörigengruppe, Neubewertung der Symptomatik und der Pflegesituation, Veränderungen der sozialen Unterstützung, und Veränderungen der Qualität der Beziehung zum Demenzkranken. Wilz folgert daraus, dass die in der Literatur postulierte herausragende Bedeutung der Bewältigungskompetenz und der sozialen Unterstützung zugunsten weiterer Faktoren relativiert werden muss. Der Aspekt der Beziehungsbiografie zwischen Pflegendem und Pflegebedürftigen spielt dabei eine besondere Rolle, finden sich doch häufig in der Beziehungsgeschichte Anhaltspunkte für die Entstehung von Schuldgefühlen beim Pflegenden.

\section{Bedeutung der Beziehungsqualität und des bisherigen Bewältigungsverhaltens für die Effektivität des Angehörigenkurses}

Die zentrale Botschaft der Fallanalysen deckt sich mit den Befunden von Lämmler (1998) und Wilz (2002): Die psychischen Mechanismen, die in Pflegebeziehungen wirksam sind, sind so tiefgreifend und komplex, dass sie sich als relativ veränderungsresistent erweisen. Es liegt nahe, dass sich diese Mechanismen modulierend auf die Effektivität einer Kursteilnahme auswirken.

In den Fallanalysen zeigte sich, dass zum einen die Beziehungsqualität zum Pflegebedürftigen, zum anderen das bisherige Bewältigungsverhalten (funktional oder dysfunktional) mitbestimmten, inwiefern Angehörige von einer Kursteilnahme profitierten (vgl. Kapitel 2.6.4). So zeigte sich in der vertiefenden Fallanalyse anhand des Beispiels von Frau M. (Kapitel 8.3.2), dass bereits vorhandene positive Bewältigungsansätze der Angehörigen durch den Kurs verstärkt werden konnten. Findet die Intervention außerdem relativ zeitnah zum Akutereignis statt, sind, wie der Fall von Frau M. zeigt, gute Einflussmöglichkeiten gegeben. Im zeitlichen Verlauf ließ sich dementsprechend eine Abnahme der Belastungswerte (Depression, Angst und körperliche Beschwerden) beobachten. 
Dagegen sind Angehörige, die ein eher dysfunktionales Bewältigungsmuster zeigen, schwer zur Teilnahme an Kursen und Gesprächsgruppen zu motivieren. Das überprotektive Beziehungsverhalten hat zur Folge, dass sich Pflegende unabkömmlich und in der Betreuung des Kranken unersetzbar fühlen. Gelingt es dennoch, Angehörige mit dysfunktionalem Bewältigungsverhalten (siehe Frau T., Kapitel 8.3.1) zu erreichen, dann muss das Veränderungspotential auf niedrigerer Stufe angesiedelt werden als bei Angehörigen mit funktionalem Bewältigungsverhalten. Die Teilnahme an einem Kurs kann dann ein erster Einstieg sein, um die Reflexionsfähigkeit der Angehörigen zu erhöhen und ihre Ressourcen zu stärken. Dies stellt eine erste notwendige Voraussetzung für die Angehörigen dar, um ihren eigenen Bedürfnissen mehr Raum zu geben und somit langfristig Veränderungen im Belastungsempfinden herbeizuführen (vgl. Jang, Clay, Roth, Haley \& Mittelman, 2004).

\section{Repertoire der Bewältigungsreaktionen bei Kursteilnehmern und Nicht- Teilnehmern}

Die Häufigkeit der Verwendung ichzentrierter bzw. patientenzentrierter Strategien unterscheidet sich grundsätzlich nicht bei Kursteilnehmern und Nicht-Teilnehmern. Insbesondere in Hinblick auf Strategien, die auf eine funktionale Bewältigung schließen lassen, sind die verwendeten Strategien jedoch sehr unterschiedlich. So ist es auffällig, dass zum Beispiel alle sechs Angehörige, die an dem Kurs teilgenommen hatten, in den Interviews angaben, professionelle Unterstützung (z. B. in Form einer Tagesstätte oder Kurzzeitpflege) in Anspruch zu nehmen. In der Kontrollgruppe gab dies nur eine der fünf Angehörigen an. In der Treatmentgruppe berichteten fünf Angehörige von Verhaltensweisen, die einen Ausgleich zur körperlichen und seelischen Belastung der

Pflege bilden, z. B. regelmäßiger Mittagsschlaf oder Spaziergang (Bewältigungsstrategie „Erholung“), in der Kontrollgruppe waren dies nur zwei. Genauso äußerten sich sechs von sechs Angehörigen der Treatmentgruppe dahingehend, dass sie die Erkrankung und die Folgen akzeptiert hätten, und zeigten damit eine realistische Einschätzung der Situation. Dagegen berichteten nur drei von fünf Angehörigen aus der Kontrollgruppe von Verhaltensweisen oder Einstellungen, die der Bewältigungsstrategie „Akzeptieren“ zuzuordnen waren. Diese Beobachtungen sind nur 
als Tendenzen aufzufassen, die Stichprobengröße der qualitativen Studie bietet keine Grundlage für eine inferenzstatistische Signifikanzprüfung. Gleichwohl bestätigen diese Tendenzen die von Wilz (2002) beschriebenen Befunde und implizieren, dass sich das Bewältigungsverhalten mit Hilfe eines Kurses positiv beeinflussen lässt.

\section{3. Überlegungen zur Forschungsmethodik: Plädoyer für eine Kombination quantitativer und qualitativer Methoden}

Für die vorliegende Untersuchung lässt sich konstatieren, dass die Kombination aus Fragebogenerhebung und Fallstudien eine differenziertere Abbildung von Prozessen des Belastungserlebens und der Belastungsverarbeitung ermöglichte, als es eine quantitative oder eine qualitative Methodik allein vermocht hätte.

Ein rein quantitativer Ansatz hätte in der vorliegenden Untersuchung einige Fragen offen gelassen, so unter anderem: Warum zeigen einige Angehörige trotz Teilnahme am Kurs keine Veränderungen im psychischen und körperlichen Wohlbefinden? Welche Prädispositionen bringen Angehörige mit, die von einer Teilnahme profitieren? Welche interaktionellen Zusammenhänge zwischen Bewältigungsverhalten und Beziehung zwischen Pflegebedürftigem und Pflegendem spielen für die Effektivität eines Angehörigenkurses eine Rolle?

Anhand der Fallstudien wurde verstehbar, mit welch unterschiedlichen Strategien der Bewältigungsarbeit die pflegenden Angehörigen versuchen, mit ihrer belastenden Situation fertig zu werden. Die zugrunde liegenden Mechanismen, die darauf Einfluss nehmen, ob und in welcher Form Angehörige Unterstützungsangebote annehmen und sich eigenen Bedürfnissen widmen, konnten erhellt werden. Durch die Einbeziehung einer qualitativen Forschungsmethodik konnten also differenziertere Erkenntnisse über die Moderatoren des Bewältigungsverhaltens, wie z. B. die Beziehungsqualität zum Pflegebedürftigen und relevante biografische Erfahrungen der Pflegeperson, gewonnen werden. Außerdem wurde die qualitative Herangehensweise an den Themenkomplex Bewältigungsstrategien eher der Komplexität des Forschungsgegenstandes und den Forderungen nach Subjektnähe und Prozessorientierung gerecht. Der Anspruch an eine Forschungsmethodik, die „eine dem persönlichen Schicksal und dem einzelnen zugewandte Methode“ (Weber, 1990) 
darstellt und die den einzelnen als „Experten seiner selbst“ würdigt (Thomae, 1968, zit. nach Weber, 1990), wurde damit erfüllt.

Gleichwohl war die Erhebung quantitativer Daten im Rahmen der Fragebogenstudie notwendig und sinnvoll. Erst die im Rahmen der quantitativen Untersuchung gewonnenen Daten ermöglichten eine gezielte und begründete Auswahl von Fällen, die ein spezifisches Belastungsprofil für eine vertiefende Analyse aufwiesen. Das Belastungsprofil (Ausmaß der Depression, Angst, körperliche Beschwerden) anhand qualitativer Verfahren $\mathrm{zu}$ ermitteln, wäre aus forschungsökonomischen Gründen nicht zu leisten gewesen.

Auch für die zukünftige Forschung im Bereich pflegende Angehörige wäre eine stärkere Hinwendung zum kombinierten Einsatz qualitativer und quantitativer Forschungsmethodik wünschenswert (siehe auch Lämmler, 1998). Gerade für Studien im Themenbereich der kritischen und chronisch belastenden Lebensereignisse empfiehlt es sich, quantitative und qualitative Methoden ergänzend und jeweils auf einander abgestimmt zu verwenden (Hearn, Lawler \& Dowswell, 2003).

Somit ist diese Arbeit nicht zuletzt als ein Plädoyer für eine stärkere Berücksichtigung pluralistischer Ansätze in der Evaluationsforschung, in der Forschungsförderung und in den einschlägigen Publikationsorganen zu verstehen. Die im Rahmen der vorliegenden Untersuchung gewonnenen Erkenntnisse ermöglichen einige erste Antworten auf die eingangs formulierten Forschungsfragen. Sie werfen jedoch auch ein Schlaglicht auf die Aspekte, in denen zukünftig noch Forschungsbedarf besteht. Diese sollen im nächsten Kapitel aufgegriffen und diskutiert werden.

\subsection{Konsequenzen für die Forschung}

„Do we need another stress and caregiving study?" fragte Zarit bereits 1989 in einem Artikel in The Gerontologist. Die Konsequenzen der Pflege können mittlerweile als recht gut erforscht angesehen werden. Und dennoch werden jedes Jahr weitere Studien veröffentlicht, die Aspekte der Konsequenzen der Pflege beleuchten (z. B. Vitaliano, Zhang \& Scanlan, 2003, im Überblick). Zukünftige Forschungsbestrebungen sollten 
sich meines Erachtens auf die folgenden zwei Themenbereichen konzentrieren: Zum einen besteht Forschungsbedarf zu der Frage, welche Bewältigungsprozesse bei der Pflege eines Angehörigen wirksam und hilfreich sind, zum anderen ist das Problem zu untersuchen, wie man adäquate Bewältigungsprozesse mit Interventionsangeboten unterstützen kann. Eine ausführliche Betrachtung der Implikationen für die zukünftige Forschung in diesen Bereichen erfolgt in den nächsten beiden Abschnitten.

\subsubsection{Implikationen für zukünftige Studien zum Thema Caregiving}

Es existieren bislang noch wenig einheitliche Ergebnisse zu Bewältigungsprozessen und deren Effektivität bei der Pflege von Schlaganfallbetroffenen. Insbesondere stellt sich die Frage, welche Copingstrategien im längeren zeitlichen Verlauf verwendet werden. Außerdem ist noch wenig darüber bekannt, inwiefern bestimmte Strategien in verschiedenen Phasen des Krankheitsprozesses vorrangig zum Einsatz kommen bzw. hilfreich sind. Dazu wäre eine Verlaufserhebung zu mehreren Zeitpunkten erforderlich. Aufgrund der Komplexität des Untersuchungsgegenstandes wäre es empfehlenswert, dabei qualitative Verfahren einzubeziehen.

Von Interesse ist außerdem die Frage, welche Verarbeitungsstrategien belastungsmindernd, welche belastungsfördernd sind. Ein zusätzlicher Erkenntnisgewinn wäre möglicherweise zu erwarten, wenn man beleuchtete, welche Copingmechanismen und äußeren Faktoren bei Personen wirksam sind, die die Pflege eines Angehörigen gut bewältigen, d. h. eine geringe Ausprägung bestimmter Belastungsmaße zeigen. Wie schon an anderer Stelle ausgeführt, kritisieren einige Autoren (z. B. Kramer, 1997a; Leipold, 2004), dass in einem Großteil der CaregivingStudien Indikatoren von adäquater psychischer Funktion vernachlässigt werden. Es überwiegen dagegen Maße für psychologische Dysfunktion (Depression, Beeinträchtigung der Gesundheit, Angst etc.). Psychische Gesundheit umfasst jedoch mehr als die reine Abwesenheit von pathologischen Elementen. Es wäre wünschenswert, wenn Dimensionen einer positiven Auffassung von psychischer Gesundheit stärkere Beachtung in der Caregiving Forschung fänden. Diese Herangehensweise, der ein salutogenetisches Modell zugrunde liegt (Antonovsky, 1997; Bengel, Strittmater, \& Willmann, 1998), könnte außerdem wichtige Impulse für die 
Weiterentwicklung von Interventionsprogrammen bieten. Wenn mehr darüber bekannt wäre, welche Faktoren zu einer gesunden und adäquaten Bewältigung der Pflege eines Angehörigen führen, könnten diese gezielter in Interventionen berücksichtigt werden, die dazu beitragen, dass Selbstüberforderung und Überfürsorglichkeit vermieden, Selbstsorge dagegen gefördert wird.

Aus der vorliegenden Untersuchung lassen sich darüber hinaus auch Themen für die Weiterentwicklung von theoretischen Modellen in der Caregiving-Forschung ableiten. Als mögliche Forschungsfrage wäre etwa die folgende denkbar: Lassen sich auf die Situation der Angehörigenpflege eher persönlichkeitsorientierte Konzepte (stabile und konsistente Bewältigungsstile oder Bewältigungsdispositionen) oder prozesshaft-dynamische Bewältigungsmodelle anwenden? Auch stellt sich die Frage nach der Generalisierbarkeit der Ergebnisse auf Angehörige von Menschen mit anderen chronischen Erkrankungen wie Krebs, Demenz oder psychischen Erkrankungen (Dinkel \& Balck, 2003; Schönberger \& von Kardorff, 2004).

Zusammenfassend lassen sich mögliche zukünftige Forschungsfragen wie folgt benennen:

- Welche Copingstrategien kommen im längeren zeitlichen Verlauf des Bewältigungsprozesses zum Einsatz?

- Welche Verarbeitungsstrategien sind belastungsmindernd, welche belastungsfördernd?

- Liegen dem Bewältigungsverhalten von pflegenden Angehörigen eher stabile und konsistente Bewältigungsstile oder eher dynamische Bewältigungsmodelle zugrunde?

- Lassen sich bestimmte Bewältigungsmuster pflegender Angehöriger einer spezifischen Patientengruppe für andere chronische Erkrankungen generalisieren?

\subsubsection{Implikationen für zukünftige Interventionsstudien}

Neben dem genannten Forschungsbedarf im Bereich der Caregiving-Forschung allgemein sind eine Verbesserung und Verfeinerung der methodischen Ansätze von 
Interventionsstudien erforderlich. Die entscheidende Kernfrage ist dabei: Wie lässt sich messen, ob eine Intervention effektiv ist?

Die Vermutung liegt nahe, dass uns dabei nicht der Ruf nach statistisch noch ausgefeilteren Studien mit Kontrollgruppen-Design und größeren Stichproben weiterbringt (Beck, 2001; Cooke et al., 2001; Pusey \& Richards, 2001), sondern eher eine Neuorientierung, was die erhobenen Outcome Variablen angeht. In Bezug auf die bislang verwendeten Messinstrumente in der Interventionsforschung lässt sich feststellen, dass der Focus eher auf den Konsequenzen des Belastungserlebens lag. Erhobene Outcome Variablen sind fast immer Angst, Depressivität etc. Es wäre wünschenswert, in zukünftigen Studien die moderierenden Faktoren stärker in den Blick zu nehmen, nämlich die Copingressourcen und die Copingstrategien. In der vorliegenden Untersuchung basieren die qualitativen Daten zu diesen Faktoren lediglich auf einmaligen Interviews, in denen Angehörige retrospektiv von ihren Bewältigungsmechanismen im Pflegeprozess berichteten. Um gezielter herausarbeiten zu können, ob und inwiefern sich Copingstrategien durch eine Intervention wie einen Angehörigenkurs verändern, wäre ein verlaufsorientiertes Forschungsdesign zu empfehlen, in dem zu mehreren Messzeitpunkten qualitative Daten zum Bewältigungsprozess erhoben werden. Als beispielhafte Studie, die einen verlaufsorientierten qualitativen Ansatz verwendet, sei hier eine Tagebuchstudie mit pflegenden Angehörigen von Demenzkranken genannt (Wilz, 2002). Weitere positive Beispiele für qualitative Forschungsansätze bei der Untersuchung von Bewältigungsstrategien lassen sich außerdem in einem verwandten Gebiet, der Psychotherapieforschung, finden (Grawe, 1999; Lambert, 2004; Lutz, 2003, 2007).

Einige Autoren fordern gar einen Paradigmenwechsel in der Angehörigeninterventionsforschung (Nolan, Ingram \& Watson, 2002; Nolan, Ryan, Enderby \& Reid, 2002): Das derzeit vorherrschende Stress- und Coping-Paradigma zielt vor allem darauf $\mathrm{ab}$, mit Hilfe von Interventionsmaßnahmen die Belastung für Pflegende zu reduzieren (Zarit, Gaugler \& Jarott, 1999). Während im Allgemeinen daran festgehalten wird, dass dies das vorrangige Ziel von Interventionen sein sollte (Clyburn, Stones, Hadjistquropoulos \& Tuckko, 2000), gibt es wachsende Bedenken, dass die Dominanz solcher Ansätze die Angehörigenpflege pathologisiert (Nolan, Ryan, Enderby \& Reid, 2002). Stattdessen sollte in Betracht gezogen werden, andere Zielparameter wie 
Lebensqualität, das Gefühl von Zufriedenheit oder Expertise in der Pflege zu verwenden, die für die Empfänger der Interventionsmaßnahme relevant und angemessen sind (Qureshi, Bamford, Nicholas, Patmore \& Harris, 2000). Qureshi und Mitarbeiter (2000) postulieren darüber hinaus, dass die Pflegeperson aktiv als Experte in den Prozess der Identifizierung und Bewertung der Outcomes einbezogen werden sollte. So soll verhindert werden, dass die Definition eines erfolgreichen Interventionsergebnisses lediglich die Sichtweise der Forscher und nicht die der Pflegenden selbst widerspiegelt (Day \& Jankey, 1996).

Außerdem sind, wie schon an anderer Stelle ausgeführt (siehe Kapitel 2.8.3 Nutzung von Unterstützungsangeboten versus Entlastungsbedarf), weitere Untersuchungen wünschenswert, die systematisch erheben, welche Hemmnisse Angehörige davon abhalten, Unterstützungsangebote wie einen Kurs überhaupt in Anspruch zu nehmen bzw. einzufordern. Neben Aspekten wie Defiziten im Informationsfluss zwischen Professionellen und Angehörigen spielen sicherlich emotionale Barrieren wie internalisierte Normen und Einstellungen oder das Leugnen von Beeinträchtigungen beim Pflegebedürftigen eine wichtige Rolle. Eine vor kurzem publizierte groß angelegte Studie in sechs europäischen Ländern untersuchte die Nutzung von Diensten und Unterstützungsangeboten und die Verfügbarkeit von finanziellen Unterstützungsmöglichkeiten in den verschiedenen Ländern. Außerdem wurden die Erfahrungen der pflegenden Angehörigen hinsichtlich der größten Hilfen bzw. Hindernisse bei der Inanspruchnahme dokumentiert. Insbesondere in den nordeuropäischen Ländern zeigt sich zwar eine hohe Verfügbarkeit von Diensten, die zum Teil aber aufgrund der geringen Anpassung an die Bedürfnisse der Pflegenden und Pflegebedürftigen nicht ausreichend genutzt werden (Lamura, Mnich, Wojszel, Nolan, Krevers, Mestheneos, \& Döhner, 2006). Eine Übertragung dieser wissenschaftlichen Erkenntnisse in die Praxis könnte helfen, Interventionsangebote besser an den Adressaten zu bringen.

Eine Frage, die in der vorliegenden Studie vernachlässigt wurde, aber möglicherweise für zukünftige Studien ebenfalls von Interesse wäre, ist folgende: Haben Interventionsmaßnahmen wie ein psychoedukativer Kurs oder Gesprächsgruppen 
für Angehörige Auswirkungen auf das Befinden und die funktionalen Fähigkeiten des Pflegebedürftigen? Diese Fragestellung ist bislang nur in wenigen Interventionsstudien zum Tragen gekommen (Haupt, Karger, Baumgärtner, Kuminoti, Jänner \& Schneider, 2000).

Die obigen Ausführungen zeigen, dass die noch zu bearbeitenden Fragen im Bereich der Caregiving- und Interventionsforschung vielfältig und komplex sind. Da die Frage nach der Pflege und Versorgung chronisch Kranker zunehmend ein Thema mit gesellschaftlicher Relevanz und Brisanz sein wird, liegt die Dringlichkeit weiterer Forschungsbemühungen auf diesem Gebiet auf der Hand.

\subsection{Implikationen für die Praxis der Angehörigenarbeit: systematische und zugehende Beratung der pflegenden Angehörigen}

Jenseits aller Vorschläge für zukünftige methodische und konzeptionelle Verbesserungen in der Forschung im Bereich der Angehörigenpflege lassen sich bereits aus den vorliegenden Untersuchungsergebnissen viele Erkenntnisse für die tägliche praktische Angehörigenarbeit in den Rehabilitationskliniken ableiten. Diese sollen im Folgenden reflektiert werden.

\section{Angehörigenarbeit zum festen Bestandteil in der Rehabilitation machen}

Bezüglich des Angebots und der Zugänglichkeit der Informationen in den Kliniken ist festzustellen, dass eine ausführliche und systematisch zugehende Beratung der Angehörigen in der Mehrzahl nicht angeboten wird (Rodgers, Bond \& Curless, 2001). Eine Kontaktaufnahme geht nach wie vor in der Regel auf die Initiative der Angehörigen zurück (Gründel, Lehmann, von Cramon \& Wilz, 2001) bzw. ergibt sich beiläufig durch die Anwesenheit des Angehörigen in der Einrichtung. Dass Mitarbeiter gezielt Kontakt zum Angehörigen suchen, geschieht oft nur, wenn Störungen im Therapieprozess auftreten und diese die Rehabilitationsziele gefährden. In den meisten Fällen haben die Professionellen eine entsprechend funktionalistische Sichtweise in Bezug auf Angehörige und sehen sie in der Rolle eines Ko-Therapeuten. Es wäre wünschenswert, wenn Fachkräfte stärker beachteten, dass dem Angehörigen eine 
Doppelrolle von Helfendem und Hilfebedürftigem zukommt. Dies erfordert auf Seiten des therapeutischen Teams einen Balanceakt: Angehörige benötigen Unterstützung, ohne dabei klientifiziert zu werden; gleichzeitig möchten sie mit ihrem Expertenwissen als Partner akzeptiert und wertgeschätzt werden. Dabei gilt es, gerade den eher dysfunktional bewältigenden Angehörigen, die ein überhöhtes pflegerisches Engagement an den Tag legen, Angebote $\mathrm{zu}$ machen. Damit Angehörigenarbeit gelingen kann, brauchen Professionelle mehr Erkenntnisse über die Situation und die Problemkonstellationen, in denen sich Angehörige befinden. Im Trialog mit Patient und Angehörigem benötigen die Fachkräfte außerdem mehr Handlungswissen. Die Dynamik zwischen allen Beteiligten des „Behandlungsdreiecks“ erfordert hohe Gesprächs- und Interventionskompetenzen, die die Fachkräfte oft nicht in ausreichendem Maße erworben haben. Die Interaktion zwischen Angehörigen und Personal ist vielfach dadurch geprägt, dass Angehörige Ärzten, Therapeuten und Pflegekräften mit hohen Erwartungen begegnen, ihnen gegenüber aber auch ein großes Ausmaß an Skepsis entgegen bringen. Gleichzeitig nehmen Fachkräfte Angehörige aufgrund ihrer hohen emotionalen Belastung und ihrer Erwartungen zum Teil als Störgrößen wahr (Schönberger \& von Kardorff, 2004). Wenn noch hinzukommt, dass die Beziehung zwischen Patient und Angehörigen konfliktbehaftet oder ambivalent ist, übertragen sich diese Störungen oft negativ auf den Kommunikationsprozess zwischen allen Beteiligten. Die Erfordernisse, die das Behandlungsdreieck Patient, Angehöriger und Fachkraft mit sich bringt, sind also sehr komplex. Die Situation und Bedürfnisse der Angehörigen und der Umgang mit ihnen sollten daher sowohl im Rehabilitationskonzept als auch in der Fort- und Weiterbildung aller in der Rehabilitation professionell Tätigen besonders berücksichtigt werden.

\section{Frühzeitige Intervention}

Interventionen - z. B. psychoedukative Kurse, individuelle Beratungsgespräche oder Unterweisungen in spezielle Pflegetechniken - sollten möglichst frühzeitig erfolgen, idealerweise während des Aufenthaltes in einer Rehabilitationseinrichtung. Dabei sollte die Form der Unterstützungsangebote angepasst sein an das Stadium des Krankheitsprozesses. In der Rehabilitation äußern Angehörige zu Beginn vor allem Informationswünsche, die sich auf allgemeine medizinische Fragen (etwa zum 
Krankheitsbild und seiner Prognose), die Vorbereitung auf die Entlassung und die Hilfsmittelversorgung beziehen (Glennon \& Smith, 1990, Rusch, Frommelt \& Thun, 1992). Die Auseinandersetzung mit den Auswirkungen der zerebralen Schädigung auf das Alltagsleben steht $\mathrm{zu}$ diesem Zeitpunkt noch im Hintergrund. Eine effektive Angehörigenarbeit muss aber sowohl die unmittelbar bedrängenden Informationsfragen beantworten als auch behutsam auf die zu erwartende Belastung nach der Entlassung vorbereiten. Einer solchen Erfordernis entsprechen Ansätze wie die von Rosenthal und Young (1988), die während der Rehabilitation allmählich den Akzent von einem psychoedukativen Vorgehen in Richtung Familienberatung oder Familientherapie verschieben. Vor dem Hintergrund der Erkenntnisse dieser Arbeit zu den Auswirkungen von Schuldgefühlen und ambivalenten Einstellungen auf das Bewältigungsverhalten erscheint eine frühzeitige Beeinflussung durch differenzierte Informationen besonders wichtig. So kann am ehesten dysfunktionalen Verarbeitungsmustern vorgebeugt werden.

\section{Zugehend und mehrstufig Kenntnisse über die Erkrankung vermitteln}

Kenntnisse über die Erkrankung bilden die notwendige Grundlage dafür, krankheitsbedingte Veränderungen des Schlaganfallbetroffenen einordnen zu können und akzeptieren zu lernen. Eine Informationsvermittlung über die Erkrankung, ihre Symptome und die zu erwartenden Belastungen schafft außerdem die Voraussetzung dafür, dass sich die Angehörigen auf den weiteren Pflegeverlauf einstellen und unterstützende Maßnahmen organisieren können. Ein psychoedukativer Kurs, so wie er in der vorliegenden Studie angeboten wurde, hat sich zur Vermittlung von Basiswissen über den Schlaganfall bewährt und sollte, trotz aller geltenden Einschränkungen, zu einem festen Bestandteil des Angebotes in Rehabilitationskliniken werden.

Der informationsvermittelnde Charakter ermuntert auch Angehörige, die einen eher patientenfixierten Bewältigungsstil an den Tag legen, zur Teilnahme, soll er doch dazu befähigen, „,dem Patienten noch besser helfen zu können“. Bewährt hat sich auch der themenzentrierte feste Veranstaltungsplan. Die Leitung sollte von einer Fachkraft mit Erfahrung in Gesprächsführung und in der Moderation von Gruppen übernommen werden, idealerweise ein Psychologe oder eine Psychologin. Das Themenspektrum der Kurseinheiten sollte wie in dem hier erprobten Kurs die Bereiche Medizin, 
Neuropsychologie, Pflege, Physiotherapie, Ergotherapie, Sprachtherapie und Sozialarbeit abdecken. Mitglieder aus den verschiedenen Berufsgruppen des Rehabilitationsteams übernehmen jeweils die Gestaltung des themenbezogenen Teils. Dabei sollte die Vermittlung der Inhalte möglichst anschaulich unter Einbeziehung praktischer Elemente erfolgen. Außerdem hat es sich bewährt, mindestens zwei Kurseinheiten dem Thema Belastung durch die Pflege und Umgang mit der Belastung zu widmen. Der besondere Vorteil bei der Durchführung von Kursen für pflegende Angehörige gegenüber der Einzelberatung liegt in der Förderung des „Lernens am Modell““. So ließ sich zum Beispiel in den hier durchgeführten Kursen beobachten, dass jüngere Teilnehmer häufig leichter professionelle Hilfen in Anspruch nehmen konnten. Ältere Teilnehmer wurden dann durch die positiven Erfahrungen ermuntert, ebenfalls psychosoziale Unterstützungsangebote anzunehmen.

Ein Kursangebot sollte immer eingebettet sein in ein mehrstufiges Interventionskonzept. Es sollte ergänzt werden durch Einzelanleitungen durch die behandelnden Therapeuten, individuelle Beratung in sozialrechtlichen Fragen, Gesprächsgruppen sowie durch ein psychotherapeutisches Angebot (Bruder, 1990; Evans, Hendricks, Haselkorn, Bishop \& Baldwin, 1992). Idealerweise sollte eine Überleitung der Angehörigen nach der Entlassung des Patienten zu einer Weiterbetreuung durch psychosoziale Dienste oder Selbsthilfevereinigungen stattfinden.

Alle Beratungs- und Informationsangebote sollten zugehender Natur sein. Gerade bei Personen mit begrenzten eigenen Unterstützungsressourcen ist oft eine aufsuchende Beratung unabdingbar. Ein Angehörigenkonzept, das aus mehreren Modulen besteht lässt sich gut an die Bedürfnisse der Angehörigen adaptieren sowie an das Ausmaß der bereits vorhandenen Bewältigungskompetenz. Leitgedanke aller Unterstützungsmaßnahmen sollte die Orientierung an vorhandenen Ressourcen sein (Rolland, 2006).

\section{Intervention an die Erwartungen und Bedürfnisse der Angehörigen anpassen}

Der Erfolg einer Intervention ist stark abhängig davon, ob die Ziele mit den Erwartungen des Angehörigen konsistent sind. Es kommt häufig vor, dass die Erwartungen von Familienangehörigen von Schlaganfallbetroffenen nicht mit der Sichtweise der behandelnden Professionellen (Ärzte, Neuropsychologen, Therapeuten, 
Sozialarbeiter) übereinstimmen. In vielen Fällen hoffen Angehörige zunächst auf eine starke Beschwerdenbesserung, wenn nicht auf Heilung (Gründel, Cramon \& Wilz, 2003). Möglicherweise hindert dies Angehörige daran, frühzeitig entsprechende Schritte zu unternehmen, die ihre Situation langfristig verbessern könnten. Bevor Angehörige bereit sind anzuerkennen, dass die Beeinträchtigungen ihres Familienmitgliedes bleibend sind, werden sie zum Beispiel nicht an einem Kurs teilnehmen, der vermittelt, dass sie sich an die veränderten Umstände ihrer Lebenssituation anpassen müssen. Dies zeigt, wie unabdingbar eine klare Kommunikation und Aufklärung ist, um Angehörigen auf adäquate Weise verständlich $\mathrm{zu}$ machen, welche Fortschritte prognostisch zu erwarten sind. Nur dann verfügen sie über eine gute Grundlage, ein entsprechendes Angebot wie ein Kursprogramm oder eine Einzelberatung in Anspruch zu nehmen. Wird dies nicht berücksichtigt, kann es geschehen, dass Angehörige Interventionsangebote gar nicht erst annehmen.

Hat man erst einmal erreicht, dass ein Angehöriger ein Kursangebot in Anspruch nimmt, sollte die Informationsvermittlung und Anleitung zur gesundheitsfördernden Lebensweise zielgruppengerecht angepasst werden. Die Bedürfnislage der jeweiligen Angehörigengruppe - ob es sich um jüngere, ältere, pflegende Ehepartner oder erwachsene Kinder, Ehemänner oder Ehefrauen handelt - sollte bei der Themenwahl und Art der Vermittlung also berücksichtigt werden.

\section{Ressourcenorientierung und Förderung eigener Lebensbereiche und Bedürfnisse}

In der Therapie von depressiven Patienten wird besonders die Förderung des „Genussverhaltens“ und der Beachtung eigener Bedürfnisse herausgestellt. Diese Aspekte sollten in der Beratung pflegender Angehöriger ebenfalls betont werden. Es ist daher außerordentlich wichtig, den Angehörigen nahe $\mathrm{zu}$ bringen, dass die Pflege eigener Bedürfnisse nicht nur legitim, sondern zwingend notwendig ist, da die langfristige Versorgung des Patienten maßgeblich von der eigenen Gesundheit und psychischen Stabilität abhängt. Daher muss auch in einem informationsvermittelnden Kurs das Thema Selbstsorge und Pflege eigener Bedürfnisse der Angehörigen ein fester Bestandteil sein.

Es empfiehlt sich außerdem ein lebensweltorientierter Ansatz für eine realistische und effektive Angehörigenarbeit. Die Probleme der Lebensführung mit der 
Krankheit müssen immer einerseits im Kontext der Bewältigung alltäglicher familiärer und beruflicher Anforderungen und andererseits in den häuslichen und milieuspezifischen Rahmenbedingungen gesehen werden (v. Kardorff \& Schönberger, 2005). Im Regelfall haben Angehörige am Ende der Rehabilitationsmaßnahme kaum Hinweise, wie sie das Leben mit der Erkrankung zu Hause bewältigen können, ohne eigene Lebensbereiche völlig aufzugeben. Hier ist es wichtig, bereits während der Rehabilitationsmaßnahme die richtigen Weichen zu stellen.

\section{Hilfestellung bei der Organisation sozialer Unterstützung}

Dieser Punkt ist eng verbunden mit dem vorgenannten Aspekt der Förderung eigener Lebensbereiche, da er eine wichtige Voraussetzung dafür darstellt. Pflegende Angehörige mit einem dysfunktionalem Bewältigungsverhalten empfinden es häufig als schwierig, Familienmitglieder oder andere Personen im Umfeld über die Pflegesituation zu informieren und um Hilfe zu bitten. Gelingt es den Angehörigen, adäquate soziale Unterstützung einzufordern, ist es auch leichter, eigene Lebensbereiche zu gestalten und damit langfristig die Belastung zu mindern. Somit stellt die Hilfestellung bei der Organisation von sozialer Unterstützung ein wesentliches Ziel der Angehörigenberatung dar. Hilfe annehmen zu lernen ist für dieses Ziel eine wesentliche Voraussetzung.

\section{Berücksichtigung individueller Wertvorstellungen, Leitsätze und der Beziehungs- biografie der Angehörigen}

$\mathrm{Ob}$ Angehörige hilfreiche Bewältigungsstrategien wie die Gestaltung eigener Lebensbereiche oder die Annahme sozialer Unterstützung anwenden, hängt eng mit ihren individuellen Wertvorstellungen, Leitsätzen und auch mit ihrer Beziehungsbiografie mit dem Pflegebedürftigen zusammen. Häufig erschweren irrationale Vorstellungen der Angehörigen die Anwendung förderlicher Bewältigungsstrategien. So sind viele pflegende Angehörige von dem inneren Leitsatz geprägt, alles, und somit auch die Pflege, alleine bewältigen zu müssen. Eine Abgabe von Pflegeaufgaben wird als Versagen erlebt und auch als ungünstig für das Befinden des erkrankten Angehörigen. Diese dysfunktionalen Kognitionen zu erkennen und zu verändern sind bedeutsame Ziele der Angehörigenarbeit. Dadurch wird die Voraussetzung dafür geschaffen, dass die Förderung eigener Lebensbereiche als 
legitimes Bedürfnis anerkannt und soziale Unterstützung als Entlastung erlebt werden kann.

Entsprechend ist eine Herangehensweise, die mögliche ungünstige und hemmende Überzeugungen thematisiert, sinnvoll. Idealerweise sollte es zum Standard einer Rehabilitationsklinik gehören, ein Anamnesegespräch mit den Angehörigen zu führen, um Risikofaktoren wie das Fehlen eines sozialen Netzwerkes, eine belastete Beziehung zum Pflegebedürftigen oder ungünstige Wertvorstellungen und innere Leitsätze zu identifizieren.

\section{Vernetzung der relevanten Institutionen im Versorgungssystem}

Einschränkend muss eingeräumt werden, dass einige der oben genannten Vorschläge zur Verbesserung der Angehörigenarbeit einen Idealzustand voraussetzen, der die personellen Möglichkeiten der meisten Einrichtungen übersteigen dürfte. So kann z. B. in den wenigsten Einrichtungen eine Weiterbetreuung der pflegenden Angehörigen nach der Entlassung geleistet werden. Umso wichtiger ist es, Vernetzungen an den Schnittstellen der medizinischen und therapeutischen Versorgung $\mathrm{zu}$ schaffen. Wünschenswert wäre, Bündnisse zwischen Selbsthilfevereinigungen, Hausärzten, Fachberatungsstellen, ambulanten Therapeuten (Physio-, Ergo- und Sprachtherapeuten, Neuropsychologen) und Rehabilitationseinrichtungen $\mathrm{zu}$ schließen. Angesichts des hohen psychosozialen Versorgungsbedarfs für Schlaganfallbetroffene und ihre Angehörigen ist eine institutionsübergreifende Koordination und Kooperation unerlässlich und sollte stärker gefördert werden. In der praktischen Umsetzung könnte dies durch Modellprojekte erprobt und weiterverbreitet werden. Projekte, die sich dieser Schnittstellenproblematik zuwenden sind jedoch bislang noch rar und nach Wissen der Autorin hauptsächlich im Bereich der Versorgung Demenzkranker zu finden.

Die vorliegende Arbeit ist als Plädoyer für eine intensivierte und systematische Angehörigenarbeit in der Rehabilitation Schlaganfallbetroffener zu verstehen. Wie die Ausgestaltung der Angehörigenarbeit idealerweise aussehen könnte, ist in den vorangegangenen Abschnitten skizziert worden. Allerdings darf bei allen Idealvorstellungen nicht der Kontext der aktuellen Entwicklungen in der Gesundheitspolitik und ihre Auswirkungen auf die Rehabilitationslandschaft außer Acht 
gelassen werden. Als besonders problematisch anzusehen ist, dass das Rehabilitationspotential bei älteren Patienten, und das sind Schlaganfallpatienten in der Regel, noch unzureichend ausgeschöpft wird. Entsprechend erreicht man in Rehabilitationseinrichtungen nur einen Bruchteil von Angehörigen, die zuhause einen Schlaganfallbetroffenen pflegen.

Das letzte Kapitel wirft ein Streiflicht auf die Rahmenbedingungen, die sich gegenwärtig hemmend auf eine adäquate Versorgung älterer Schlaganfallpatienten in Deutschland auswirken und damit auch eine flächendeckende effektive Angehörigenarbeit erschweren.

\subsection{Ausblick}

Gegenwärtig stellt der Schlaganfall in Deutschland die häufigste Ursache für eine lebenslange Behinderung im Erwachsenenalter dar, sodass diesem Krankheitsbild auch in den nächsten Jahrzehnten eine herausragende sozialmedizinische und gesundheitsökonomische Bedeutung zukommen wird. Grundsätzlich sind in den vergangenen Jahren große Fortschritte in der Verbesserung der Akutversorgung, der Frührehabilitation und auch der langfristigen Rehabilitation nach Schlaganfall erzielt worden. Vor dem Hintergrund der demografischen Entwicklung in Verbindung mit sozialepidemiologischen Analysen von (Multi-)Morbidität wird aber der Bedarf an qualifizierter Akutversorgung und Rehabilitation insbesondere von älteren Schlaganfallpatienten weiter zunehmen (Steinhagen-Thiessen \& Borchelt, 1996). Zwar mangelt es nicht an fundierten Konzepten zur geriatrischen Rehabilitation; neben den Empfehlungen der Bundesarbeitsgemeinschaft der Klinisch-Geriatrischen Einrichtungen bestehen von einzelnen Bundesländern ausgearbeitete Geriatriekonzepte (siehe Wagner, 2004). Auch ist schon seit 2001 mit der Neuregelung des Sozialgesetzbuches (SGB) IX auf politischer Ebene festgeschrieben worden, dass medizinische Rehabilitationsmaßnahmen vorrangig vor Pflegeleistungen $\mathrm{zu}$ ergreifen sind. Der oft zitierte Leitsatz „Rehabilitation vor Pflege“ wird in der Realität jedoch keineswegs flächendeckend umgesetzt (v. Kardorff, 1999; Sachverständigenrat zur Begutachtung der Entwicklung im Gesundheitswesen, 2005). Schwerpunkt der Rehabilitationsbemühungen der Leistungsträger ist immer noch die 
Wiedereingliederung jüngerer Schlaganfallpatienten in das Erwerbsleben. Dies ist in erster Linie volkswirtschaftlichen Gesichtspunkten geschuldet. Die rechtzeitige und fachgerechte Rehabilitation älterer Schlaganfallpatienten wird durch vielfältige systembedingte Zuständigkeits- und Finanzierungsprobleme erschwert (Bundesministerium für Familie, Senioren, Frauen und Jugend [BFSFJ], 2002): Zum einen verursacht die sektorale Trennung zwischen Krankenhausbehandlung nach $§ 39$ Abs. I SGB V und der medizinischen Rehabilitation nach $\S 40$ SGB V in der Praxis oft Auslegungs- und Zuordnungsprobleme. Dies hängt mit der Komplexität der geriatrischen Behandlung zusammen, die durch das multidisziplinäre Zusammenwirken u. a. von Pflegekräften, Therapeutinnen und Therapeuten, Sozialarbeit und ständiger verantwortlicher ärztlicher Präsenz gekennzeichnet ist. Zum anderen sind die Krankenkassen als Rehabilitationsträger nicht für die Pflegeleistungen zuständig, womit sich eine „Ersparnis“ bei erfolgreich durchgeführter Rehabilitation also nicht bei ihnen niederschlägt, sondern bei den finanziell getrennt verwalteten Pflegekassen. Damit fehlt bei den Krankenkassen ein entsprechender finanzieller Anreiz, Rehabilitation zu bewilligen. Dies lässt berechtigterweise die Frage aufkommen, ob die Trennung der Krankenversicherung von der Pflegeversicherung die sinnvolle Umsetzung des Grundsatzes „Rehabilitation vor Pflege“ erschwert oder sogar verhindert (Sachverständigenrat für die Konzertierte Aktion im Gesundheitswesen, 2001).

Was bedeuten diese Ausführungen für das Anliegen der vorliegenden Arbeit, Angehörige von Schlaganfallpatienten mit Hilfe eines psychoedukativen Programms zu einer effektiveren Bewältigung der Pflege zu befähigen? Will man an dem sowohl unter ökonomischen wie auch ethischen Gesichtspunkten wünschenswerten Grundsatz festhalten, dass die Pflege chronisch kranker Menschen vorrangig im familiären Umfeld stattfinden sollte, bedarf es mehr, als eine optimale Angehörigenarbeit in einigen wenigen Modelleinrichtungen $\mathrm{zu}$ betreiben. Dies geht an den tatsächlichen Gegebenheiten vorbei, da in Rehabilitationseinrichtungen nur ein Bruchteil der Angehörigen erreicht werden, die ältere Schlaganfallpatienten zu Hause versorgen (Sachverständigenrat zur Begutachtung der Entwicklung im Gesundheitswesen, 2005). Denkbar wäre eine grundsätzliche Angliederung von Angehörigenarbeit an andere Institutionen (z. B. Selbsthilfeorganisationen oder Beratungsstellen). 
Rehabilitationseinrichtungen sind jedoch meines Erachtens besser geeignet, eine effektive Angehörigenarbeit zu leisten. Zum einen bestehen die notwendigen fachlichen, strukturellen und organisatorischen Voraussetzungen, zum anderen lässt sich eine bessere Verzahnung der Versorgung des Patienten mit der Einbindung der Angehörigen erreichen. Zudem ist ein zugehendes Konzept der Angehörigenarbeit leichter im Rahmen einer Rehabilitationseinrichtung zu verwirklichen. Wünschenswert wäre also eine weitere Verbesserung der Rehabilitationsangebote für ältere Schlaganfallpatienten. Abhilfe könnte zum einen der flächendeckende Ausbau eines abgestuften (geriatrischen) Versorgungskonzeptes schaffen, das ambulante, teilstationäre und stationäre Versorgungsinstitutionen integrativ miteinander verbindet. Die Übergänge zwischen diesen Bereichen müssen mit möglichst geringem Verlust an Informationen über die Patientinnen und Patienten und unter Vermeidung von doppeltem diagnostischem Aufwand ermöglicht werden. Insbesondere sollte eine stärkere Förderung des ambulanten Bereichs angestrebt werden, da dieser kosteneffektiver organisiert werden kann (Borchelt \& Steinhagen-Thiessen, 2001). Hinzu kommt, dass mit der höheren Alltagsnähe in der ambulanten rehabilitativen Versorgung eine höhere Lebensqualität für den Patienten erreicht werden kann.

In kommenden Jahren bedarf es weiterer wissenschaftlich fundierter Studien, um die Erfolge zu untermauern, die geriatrische Rehabilitation zeitigt (Meier-Baumgartner et al., 2002). Nur mit Hilfe evidenzbasierter Argumente wird es gelingen, der geriatrischen Rehabilitation im Wettbewerb um die begrenzten Ressourcen im Gesundheitswesen eine gute Ausgangsposition zu sichern. Kostenträger verlangen immer häufiger Qualitätskontrollen. Die Wiedererlangung der größtmöglichen Selbständigkeit im Alltag und Verbesserung von Lebensqualität und Wohlbefinden für den Patienten soll mit wirtschaftlichem Einsatz von Mitteln erreicht werden. Dies ist ein legitimes, wenn auch schwieriges Postulat bei einem hochkomplexen Gebiet wie der geriatrischen Rehabilitation.

Mit dem Ausbau eines abgestuften integrierten Versorgungskonzeptes in der Schlaganfallrehabilitation, das mit einem ganzheitlichen Ansatz auch die Angehörigen einbezieht, kann es meines Erachtens am ehesten gelingen, längerfristig Kosten einzusparen bei verbesserter Lebensqualität für Betroffene wie Angehörige. 


\section{Literatur}

Adler, C., Gunzelmann, T., Machold, C., Schumacher, J., Wilz, G. (1996). Belastungserleben pflegender Angehöriger von Demenzpatienten. Zeitschrift für Gerontologie und Geriatrie, 29, 143-149.

Aneshensel, C.S., Mullan, J.T., Pearlin, L.I., Zarit, S.H. \& Whitlatch, C.J. (1995). Profiles in caregiving: the unexpected career. New York: Academic Press.

Anthony-Bergstone, C.R., Zarit, S.H. \& Gatz, M. (1988). Symptoms of psychological distress among caregivers of dementia patients, Psychology and Aging, 3, 245248.

Antonovsky, A. (1997). Salutogenese: Zur Entmystifizierung der Gesundheit. Tübingen: DGVT-Verlag.

Antonucci, T.C. \& Jackson, J.S. (1987). Social support, interpersonal efficacy, and health: A life course perspective. In L. Carstensen \& B.A. Edelstein (Eds.), Handbook of clinical gerontology (pp. 291-311). Elmsford, NY: Pergamon Press.

Arbeitsgruppe Geriatrisches Assessment (AGAST) (Hrsg.) (1995). Geriatrisches Basisassessment: Handlungsanleitung für die Praxis. München: MMV, MedizinVerlag.

Auer, S., Donabauer, Y., Zehetner, F. \& Span, E. (2007). Entlastung pflegender Angehöriger. Ein Programm der M.A.S. Alzheimerhilfe. Zeitschrift für Gerontopsychologie und -psychiatrie, 20, 169-174.

Bamford, J., Sandercock, P., Dennis, M., Warlow, C., Jones, L., McPherson, K., Vessey, M., Fowler, G., Molyneux, A., Hughes, T., Burn, J. \& Wade, D. (1988). A prospective study of acute cerebrovascular disease in the community: The Oxfordshire Stroke Project 1981-1986. 1. Methodology, demography and incident cases of first year after stroke. Journal of Neurology, Neurosurgery and Psychiatry, 51, 1373-1380.

Barber, C.E. (1988). Correlates of subjective burden among adult sons and daughters caring for aged parents. Journal of Aging Studies, 2, 133-144.

Barusch, A.S. \& Spaid, W.M. (1989). Gender differences in caregiving: Why do wives report greater burden? The Gerontologist, 29, 667-676.

Beach, D.L. (1997). Family caregiving: The positive impact on adolescent relationships. The Gerontologist, 37, 233-238.

Beck, C. (2001). Identification and assessment of effective services and interventions: The nursing home perspective. Aging and Mental Health, 5, 99-111.

Beck, A.T., Ward, C.H., Mendelson, M., Mock, J. \& Erbaugh, J. (1961). An inventory for measuring depression. Archives of General Psychiatry, 4, 561-571.

Bédard, M., Kuzik, R., Chambers, L., Molloy, W., Dubois, S. \& Lever, J. (2005). Understanding burden differences between men and women caregivers: the contribution of care-recipient problem behaviors. International Psychogeriatrics, 17, 99-118.

Bédard, M., Molloy, D.W., Pedlar, D., Lever, J. \& Stones, M. (1997). Associations between dysfunctional behaviours, gender, and burden in spousal caregivers of cognitively impaired older adults. International Psychogeriatrics, 9, 277-290. 
Bengel, J., Strittmater, R. \& Willmann, H. (1998). Was erhält Menschen gesund? Antonovskys Modell der Salutogenese - Diskussionsstand und Stellenwert. Köln: BZgA, Reihe Forschung und Praxis der Gesundheitsförderung, Band 6.

Berger, K., Kolominsky-Rabas, P., Heuschmann, P. \& Keil, K. (2000). Die Häufigkeit des Schlaganfalls in Deutschland. Prävalenzen, Inzidenzen und ihre Datenquellen. Deutsche Medizinische Wochenschrift, 125, 21-25.

Berliner Service- und InformationsZentrum für Angehörigenarbeit (BeSIZ) (1998). Sachbericht des BeSIZ. Berlin.

Berry, G.L., Zarit, S.H. \& Rabatin, V.X. (1991). Caregiver activity on respite and nonrespite days: A comparison of two service approaches. The Gerontologist, 31, $830-835$.

Besche, A. (2000). Die Pflegeversicherung: Textausgabe mit einer ausführlichen Einführung. Köln: Bundesanzeiger.

Biegel, D.E. \& Blum, A. (1990). Aging and caregiving. Theory, research, and policy. Newbury Park: Sage.

Biegel, D.E., Sales, E. \& Schulz, (1991). Family caregiving in chronic illness: Alzheimer's disease, cancer, heart disease, mental illness, and stroke. Newbury Park, CA: Sage.

Binder, L.M. (1983). Emotional problems after stroke. Current Concepts of Cerebrovascular Disease. Stroke, 18, 17-21.

Bishop, D.S. \& Evans, R.L. (1995). Families and stroke: The clinical implications of research findings.Topics in Stroke Rehabiltiation, 2, 20-31.

Blieszner, R. \& Hamon, R.R. (1992). Filial responsibility. In: J.W. Dwyer \& R.T. Coward (Eds.), Gender, families, and elder care (pp. 105-119). Newbury Park, CA: Sage Publications, Inc.

Blinkert, B. \& Klie, T. (2004). Gesellschaftlicher Wandel und demographische Veränderungen als Herausforderungen für die Sicherstellung der Versorgung von pflegebedürftigen Menschen. Sozialer Fortschritt, 43, 319-325.

Bodnar, J.C. \& Kiecolt-Glaser, J.K. (1994). Caregiver depression after bereavement: Chronic stress isn't over when it's over. Psychology and Aging, 9, 372-380.

Boeger, A. \& Pickartz, A. (2001). Bewältigungsstrategien bei pflegenden Angehörigen. Report Psychologie, 26, 234-239.

Bolger, N., DeLongis, A., Kessler, R. \& Schilling, E. (1989). Effects of daily stress on negative mood. Journal of Personality and Social Psychology, 46, 877-891.

Bond, J. (2000). The impact of staff factors in nursing home residents. Aging and Mental Health, 4, 5-8.

Boniface, S. \& Ziemann, U. (Eds.) (2003). Plasticity in the human nervous system: scientific and clinical studies with TMS. Cambridge: University Press.

Bookwala, J., Zdaniuk, B., Burton, L., Lind, B., Jackson, S. \& Schulz, R. (2004). Concurrent and long-term predictors of older adults' use of community-based longterm care services: The caregiver health effects study. Journal of Aging and Health, $16,88-115$.

Borchelt, M. \& Steinhagen-Thiessen, E. (2001). Ambulante geriatrische Rehabilitation - Standortbestimmung und Perspektiven. Zeitschrift für Gerontologie und Geriatrie, 34, 24-29.

Borden, W. \& Berlin, S. (1990). Gender, coping and psychological well-being in spouses of older adults with chronic dementia. American Journal of Orthopsychiatry, 60, 603-610. 
Brashares, H. \& Catanzaro, S.J. (1994). Mood regulation expectancies, coping responses, depression and sense of burden in female caregivers of Alzheimer's patients. Journal of Nervous and Mental Disease, 182, 437-442.

Bridges, L. (2003). Coping as an element of developmental well-being. In M.H. Bornstein, L. Davidson, C. Keyes, K. Moore, (Eds.), Well-being: Positive development across the life course. London: Lawrence Erlbaum.

Brodaty, H. (1992). Carers: training informal carers. In T. Arie (Ed.), Recent Advances in Psychogeriatrics, (pp. 163-171). Churchill Livingstone.

Brodaty, H. (2005). Psychogeriatric service delivery: An international perspective. Oxford: University Press.

Brodaty, H. \& Gresham, M. (1992). Prescribing residential respite care for dementia: Effects, side effects, indications and dosage. International Journal of Geriatric Psychiatry, 7, 357-362.

Brody, E.M. (1981). Women in the middle and family help to older people. The Gerontologist, 21, 471-480.

Bruder, J. (1988). Filiale Reife - ein wichtiges Konzept für die familiäre Versorgung kranker, insbesondere dementer alter Menschen. Zeitschrift für Gerontopsychologie und -psychiatrie, 1, 95-101.

Bruder, J. (1990). Alterspsychotherapie und Angehörigenarbeit. Zeitschrift für Gerontopsychologie und - psychiatrie, 3, 130-137.

Bruder, J. (1998). Beratung und Unterstützung von pflegenden Angehörigen demenzerkrankter Menschen. In: A. Kruse (Hrsg.), Psychosoziale Gerontologie, Bd. 2: Intervention, (S. 275-295). Göttingen: Hogrefe.

Bruhn, J. \& Phillips, B. (1984). Measuring social support: A synthesis of current approaches. Journal of Behavioral Medicine, 7, 151-169.

Bundesarbeitsgemeinschaft der Beratungsstellen für alte Menschen und ihre Angehörigen (BAGA) (Hrsg.) (1999). Handbuch Demenz. www.baga.de

Bundesarbeitsgemeinschaft für Rehabilitation BAR (Hrsg.) (2005). Rahmenempfehlungen zur ambulanten neurologischen Rehabilitation. www.barfrankfurt.de

Bundesministerium für Familie und Senioren (Hrsg.) (1993). Hilfe- und Pflegebedürftige in privaten Haushalten. Schriftenreihe des Bundesministeriums für Familie und Senioren, Band 20.2. Stuttgart: Kohlhammer.

Bundesministerium für Familie, Senioren, Frauen und Jugend (Hrsg.) (2002). Vierter Altenbericht zur Lage der älteren Generation in der Bundesrepublik Deutschland: Risiken, Lebensqualität und Versorgung Hochaltriger - unter besonderer Berücksichtigung demenzieller Erkrankungen. Berlin.

Burgio, L., Stevens, A., Guy, Roth, D. \& Haley, W. (2003). Impact of two psychosocial interventions on white and African American family caregivers of individuals with dementia. The Gerontologist, 43, 568-579.

Burdz, M.P., Eaton, W.O. \& Bond, J.B. (1988). Effect of respite care on dementia and nondementia patients and their caregivers. Psychology and Aging, 3, 38-42.

Busch, E. \& Diener, H.C. (2003). Schlaganfallversorgung - mit Netzwerken auf innovativen Wegen. Deutsches Ärzteblatt, 100, 2567-2569.

Cantor, M.H. (1983). Strain among caregivers: A study of experience in the United States. The Gerontologist, 23, 597-604. 
Carver, C.S. \& Scheier, M.S. (2003). Three human strengths. In L.G. Aspinwall \& U. M. Staudinger (Eds.), A psychology of human strengths: Fundamental questions and future directions for a positive psychology, (pp. 87-102). Washington: APA.

Caserta, M.S., Lund, D.A., Wright, S.D. \& Redburn, D.E. (1987). Caregivers to dementia patients: The utilization of community services. The Gerontologist, 27, 209-214.

Cattanach, L. \& Kraemer Tebes, J. (1991). The nature of elder impairment and its impact on family caregivers' health and psychosocial functioning. The Gerontologist, 31, 246-255.

Chenoweth, B. \& Spencer, B. (1986). Dementia: The experience of family caregivers. The Gerontologist, 26, 267-272.

Choi, T., Lavohn, J., Christensen, M. (1983). Health specific family coping index for noninstitutional care. American Journal of Public Health, 73, 1275-1277.

Chwalisz, K. (1992). Perceived stress and caregiver burden after brain injury: a theoretical integration. Rehabilitation Psychology, 37, 189-203.

Cicirelli, V.G. (1991). Adult children's help to aging parents: Attachment and altruism. In: L. Montada \& H.W. Bierhoff (Eds.), Altruism in Social Systems, (pp. 42-57), Göttingen: Hogrefe.

Cicirelli, V.G. (1993). Attachment and obligation as daughters' motives for caregiving behavior and subsequent effect on subjective burden. Psychology and Aging, 8, 144-155.

Clipp, E.C. \& George, L.K. (1990). Psychotropic drug use among caregivers of patients with dementia. Journal of the American Geriatric Society, 38, 227-235.

Clipp, E.C. \& George, L.K. (1993). Dementia and cancer: A comparison of spouse caregivers. The Gerontologist, 33, 534-541.

Clyburn, L.D., Stones, M.J., Hadjistquropoulos, T. \& Tuccko, H. (2000). Predicting caregiver burden and depression in Alzheimer's disease. Journal of Gerontology, Social Sciences, 5SB, S2-S13.

Coates, D., Renzaglia, G.J. \& Embree, M.C. (1983). When helping backfires: Help and helplessness. In J.D. Fisher, A. Nadler \& B.M. DePaulo (Eds.), New directions in helping: Voll. Recipient reaction to aid (pp. 251-279). New York: Academic.

Coen, R., Swanwick, G., O'Boyle, C. \& Coakley, D. (1997). Behavior disturbance and other predictors of carer burden in Alzheimer's disease. International Journal of Geriatric Psychiatry, 12, 331-336.

Cohen, D. (2000). Caregivers for persons with Alzheimer's disease. Current Psychiatry Reports, 2, 32-39.

Cohen, D. \& Eisdorfer, C. (1988). Depression in family members caring for a relative with Alzheimer's disease. Journal of the American Geriatric Society, 36, 885-889.

Cohen, C.A., Gold, D.P., Shulman, K.I. \& Zucchero, C.A. (1994). Positive aspects in caregiving: An overlooked variable in research. Canadian Journal on Aging, 13, 378-391.

Cooke, D.D., McNally, L., Mulligan, K.T., Harrison, M.J.G. \& Newman, S.P. (2001). Psychosocial interventions for caregivers of people with dementia: a systematic review. Aging \& Mental Health, 5, 120-135.

Coon, D.W., Gallagher-Thompson, D., Thompson, L. (2003). Innovative interventions to reduce dementia caregiver distress: A clinical guide. New York: Springer. 
Coon, D.W., Thompson, L., Steffen, A., Sorocco, K. \& Gallagher-Thompson, D. (2003). Anger and depression management: Psychoeducational skill training interventions for women caregivers of a relative with dementia. The Gerontologist, 43, 678-689.

Corbin, J. \& Strauss, A. (1990). Grounded theory research: Procedures, canons, and evaluative criteria. Qualitative Sociology, 13, 3-21.

Davis, L. (1992). Building a science of caring for family caregivers. Family and Community Health, 15, 1-9.

Day, A. \& Jankey, S.G. (1996). Lessons form the literatures: Towards a holistic model of quality for life. In R. Renwick, I. Brown \& M. Nagler (Eds.) Quality of life in health promotion and rehabilitation: Conceptual approaches, issues and applications. Thousand Oaks, CA: Sage.

Deimling, G.J. \& Bass, D.M. (1986). Symptoms of mental impairment among elderly adults and their effects on family caregivers. Journal of Gerontology, 41, 778-784.

Deimling, G.T., Bass, D.M., Townsend, A.L. \& Noelker, L.S. (1989). Care-related stress: A comparison of spouse and adult-child caregivers in shared and separate households. Journal of Aging and Health, 1, 67-82.

Denman, A. (1998). Determining the needs of spouses caring for aphasic partners. Disability Rehabilitation, 20, 411-423.

Dennis, M., O’Rourke, S., Lewis, S., Sharpe, M. \& Warlow, C. (1998). A quantitative study of the emotional outcome of people caring for stroke survivors. Stroke, 29, $1867-1872$.

Denzin, N.K. (1989). The research act (3rd ed.). New York: McGraw-Hill.

Denzin, N.K. \& Lincoln, Y.S. (1994). Introduction: Entering the field of qualitative research. In N.K. Denzin \& Y.S. Lincoln (Eds.), Handbook of qualitative research, (pp. 1-17). Thousand Oaks: Sage.

Denzler, P. (1994a). Kommunikationsstörungen nach Schlaganfall und ihre Behandlung. In: K.-H. Mauritz (Hrsg.), Rehabilitation nach Schlaganfall (S. 175199). Stuttgart: Kohlhammer.

Denzler, P. (1994b). Neuropsychologische Ausfälle nach Schlaganfall. In: K.-H. Mauritz (Hrsg.), Rehabilitation nach Schlaganfall (S.142-174). Stuttgart: Kohlhammer.

Diener, H.C. \& Hacke, W. (Hrsg.) (2002). Leitlinien für Diagnose und Therapie in der Neurologie. Stuttgart: Thieme.

Diener, H.C., Hacke, W. \& Forsting, M. (Hrsg.) (2004). Schlaganfall. Stuttgart: Thieme.

Dilworth-Anderson, P., Goodwin, P. \& Williams, S. (2004). Can culture help explain the physical health effexts of caregiving over time among African American caregivers? Journal of Gerontology, 59, S138-S145.

Dinkel, A. \& Balck, F. (2003). Krankheit, Rehabilitation und Familie. Praxis Klinische Verhaltensmedizin und Rehabilitation, 62, 116-121.

Döhnert, M., Wilz, G., Adler, C., Gunzelmann, T. \& Brähler, E. (2001). Zusammenhänge von Salivacortisol und Befindlichkeit - Eine quantitative Einzelfallanalyse an zwei pflegenden Angehörigen von Demenzkranken. Zeitschrift für Klinische Psychologie, Psychiatrie und Psychotherapie, 49, 140-165.

Donaldson, C., Tarrier, N. \& Burns, A. (1997). The impact of symptoms of dementia on caregivers. British Journal of Psychiatry, 170, 62-68. 
Donaldson, C., Tarrier, N. \& Burns, A. (1998). Determinants of carer stress in Alzheimer's disease. International Journal of Geriatric Psychiatry, 13, 248-256.

Dowswell, G., Lawler, J. \& Young, J. (2000). Unpacking the "black box" of a nurse-led stroke support service. Clinical Rehabilitation, 14, 160-171.

Draper, B., Bowring, G., Thompson, C., Van Heyst, J., Conroy, P. \& Thompson, J. (2007). Stress in caregivers of aphasic stroke patients: a randomized controlled trial. Clinical Rehabiltiation, 21, 122-130.

Draper, B.M., Poulos, C.J., Cole, A.M., Poulos, R.G. \& Ehrlich, F. (1992). A comparison of caregivers for elderly stroke and dementia victims. Annual National Conference of the Australian Association of Gerontology. Journal of the American Geriatrics Society, 40, 896-901.

Dunkel-Schetter, C. \& Bennett, T.L. (1990). Differentiating the cognitive and behavioral aspects of social support. In: B.R. Sarason, I.G. Sarason \& G.R. Pierce, Social support: an interactional view (pp. 267-296), New York: Wiley.

Dura, J.R., Stukenberg, K.W. \& Kiecolt-Glaser, J.K. (1991). Anxiety and depressive disorders in adult children caring for demented parents. Psychology and Aging, 6, 467-473.

Dwyer, J.W., Lee, G.R. \& Jankowski, T.B. (1994). Reciprocity, elder satisfaction, and caregiver stress and burden: The exchange of aid in the family caregiving relationship. Journal of Marriage and the Family, 56, 34-43.

Elliott, G.R. \& Eisdorfer, C. (1982). Stress and human health. New York: Springer.

Enright, R.B. (1991). Time spent caregiving and help received by spouses and adultchildren of brain-impaired adults. The Gerontologist, 31, 375-383.

Evans, R.L. \& Baldwin, D. (1989). Factors affecting decision to participate in caregiver support groups. Archives of Physical Medicine and Rehabilitation, 70, 87-93.

Evans, R.L. \& Held, S. (1984). Evaluation of family stroke education. International Journal of Rehabilitation Res., 7, 47-51.

Evans, R.L., Hendricks, R.D., Haselkorn, J.K., Bishop, D.S. \& Baldwin, D. (1992). The family's role in stroke rehabilitation. American Journal of Physical Medicine and Rehabilitation, 71, 135-139.

Evans, R. L., Matlock, A.L., Bishop, D. S., Stranahan, S. \& Pederson, C. (1988). Family intervention after stroke: does counselling or education help? Stroke, 19, 12431249.

Farran, C. (1997). Theoretical perspectives concerning positive aspects of caring for elderly persons with dementia: Stress/adaptation and existentialism. The Gerontologist, 37, 250-256.

Farran, C., Keane-Hagerty, E., Salloway, S., Kupferer, S. \& Wilken, C.S. (1991). Finding meaning: An alternative paradigm for Alzheimer's disease family caregivers. The Gerontologist, 31, 483-489.

Fitting, M., Rabins, P., Lucas, M.J. \& Eastham, J. (1986). Caregivers for dementia patients: A comparison of husbands and wives. The Gerontologist, 26, 248-252.

Flick, U. (1990). Fallanalysen: Geltungsbegründung durch systematische PerspektivenTriangulation. In G. Jüttemann (Hrsg.), Komparative Kasuistik (S. 184-203). Heidelberg: Asanger.

Folkman S. \& Lazarus, R.S. (1980). An analysis of coping in a middle aged community sample. Journal of Health and Social Behavior, 21, 219-239. 
Folstein, M.F., Folstein, S.E. \& McMugh, P.R. (1975). „Mini-Mental State“: A practical method for grading the cognitive state of patients for the clinician. Journal of Psychiatry Research, 12, 189-198.

Forster, L.E. \& Stoller, E.P. (1992). Impact of social support on mortality: A seven-year follow-up of older men and woman. Journal of Applied Gerontology, 11, 173-186.

Franke, L. (2000). Psychosoziale Beratung für Angehörige Demenzkranker. Erste Konturen der unterschiedlichen Nutzerprofile von Ehegatten und Kindern. Band 24, Kasseler Gerontologische Schriften.

Franks, M.M. \& Stephens, M.A.P. (1992). Multiple roles of middle-generation caregivers: Contextual effects and psychological mechanisms. Journal of Gerontology: Social Sciences, 47, 123-129.

Fredman, L., Tennstedt, S., Smyth, K., Kasper, J., Miller, B., Fritsch, Watson, M. \& Harris, E. (2004). Pragmatic and internal validity issues in sampling in caregiver studies. Journal of Aging and Health, 16, 175-203.

Friedrichs, J. (1990). Methoden empirischer Sozialforschung. Opladen: Westdeutscher Verlag.

Fromm, M. (1990). Zur Verbindung quantitativer und qualitativer Methoden. Pädagogische Rundschau, 44, 469-481.

Gallant, M. \& Connell, C. (1997). Predictors of decreased self-care among spouse caregivers of older adults with dementing illness. Journal of Aging and Health, 9, 373-395.

Garz, D. \& Kraimer, K. (1991). Qualitativ-empirische Sozialforschung im Aufbruch. In D. Garz \& K. Kraimer (Hrsg.) Qualitativ-empirische Sozialforschung: Konzepte, Methoden, Analysen (S. 1-33). Opladen: Westdeutscher Verlag.

Gatz, M., Bengtson, V.L. \& Blum, M.J. (1990). Caregiving families. In J.E. Birren \& K.W. Schaie (Eds.), Handbook of the Psychology of Aging (S. 404-426). New York: Van Nostrand.

Gaugler, J., Kane, R. \& Newcomer, R. (2007). Resilience and transitions from dementia caregiving. Journals of Gerontology, Psychological Sciences, 62, 38-44.

Gauggel, S., Schmidt, A. \& Didié, M. (1994). Körperliche Beschwerden und deren Einfluß auf die Erfassung depressiver Störungen bei jüngeren und älteren Menschen. Zeitschrift für Gerontopsychologie und-psychiatrie, 7, 203-210.

George, L.K. (1994). Caregiver burden and well-being: An elusive distinction. The Gerontologist, 34, 6-7.

George, L.K. \& Gwyther, L.P. (1986). Caregiver well-being: A multidimensional examination of family caregivers of demented adults. The Gerontologist, 26, 253259.

Gilhooly, M.L.M. (1984). The impact of caregiving on caregivers: Factors associated with psychological well-being of people supporting a dementing relative in the community. British Journal of Medical Psychology, 57, 35-44.

Given, C., Given, B., Stommel, M. \& Azzouz, F. (1999). The impact of new demands for assistance on cargiver depression: Tests using an inception cohort. The Gerontologist, 39, 76-85.

Glass, T., Berkman, L., Hiltunen, E., Furie, K., Glymour, M., Fay, M. \& Ware, J. (2004). The families in recovery from stroke trial (FIRST): primary study results. Psychosomatic Medicine, 66, 889-897.

Gleixner, C., Müller, M. \& Wirth, S. (1999). Neurologie und Psychiatrie für Studium und Praxis. 2. Auflage. Breisach: Med. Verlags- und Informationsdienste. 
Glennon, T. \& Smith, B.S. (1990). Questions asked by patients and their support groups during family conferences on inpatient rehabilitation units. Archives of Physical Medicine and Rehabilitation, 71, 699-702.

Glosser, G. \& Wexler, D. (1985). Participants' evaluation of educational/support groups for families of patients with Alzheimer's diesease and other dementias. The Gerontologist, 25, 232-238.

Gold, D.P., Cohen, C., Shulman, K., Zucchero, C., Andres, D. \& Etzezadi, J. (1995). Caregiving and dementia: Predicting negative and positive outcomes for caregivers. International Journal of Aging and Human Development, 41, 183-201.

Gottlieb, B.H. \& Wolfe, J. (2002). Coping with family caregiving to persons with dementia: a critical review. Aging and Mental Health, 6, 325-342.

Gräßel, E. (1997). Angehörigenberatung wichtige hausärztliche Funktion. Der Allgemeinarzt, 16, 1506-1510.

Gräßel, E. (1998). Belastung und gesundheitliche Situation der Pflegenden. Querschnittuntersuchung zur häuslichen Pflege bei chronischem Hilfs- oder Pflegebedarf im Alter (2. Aufl.). Egelsbach: Hänsel-Hohenhausen.

Grafstrom, M., Fratiglioni, L., Sandman, P.O. \& Winblad, B. (1992).Health and social consequences for relatives of demented and non-demented elderly. A populationbased study. Journal of Clinical Epidemiology, 45, 861-870.

Granger, C.V., Albrecht, G.L., Hamilton, B.B. (1979). Outcome of comprehensive medical rehabilitation: measurement by PULSES profile and the Barthel Index. Archives of Physical Medicine and Rehabilitation, 61, 355-358.

Grant, J.S., Elliott, T.R., Weaver, M., Bartolucci, A.A. \& Giger, J.N. (2002). Telephone intervention with family caregivers of stroke survivors after rehabilitation. Stroke, 33, 2060-2065.

Grant, J.S., Weaver, M., Elliott, T.R., Bartolucci, A.A. \& Newman-Giger, J.N. (2004). Family caregivers of stroke survivors: Characteristics of caregivers at risk for depression. Rehabilitation Psychology, 49, 172-179.

Grawe, K. (1999). Wie kann Psychotherapie noch wirksamer warden? Verhaltenstherapie und psychosoziale Praxis, 31, 185-199.

Greene, V.L. \& Monahan, D.J. (1989). The effect of a support and education program on stress and burden among family caregivers to frail elderly persons. The Gerontologist, 29, 472-477.

Gründel, A., Cramon, D.Y. v. \& Wilz (2003). „Ich hoffe, dass es wieder wird wie vorher...": Prospektive Annahmen von Paitenten und Angehörigen über die Folgen des Schlaganfalls zu Beginn der Rehabilitation. Praxis Klinische Verhaltensmedizin und Rehabilitation, 62, 157-170.

Gründel, I., Lehmann, A., Cramon, D.Y. von \& Wilz, G. (2001). Der Unterstützungsbedarf von Angehörigen während der stationören Rehabilitation von Schlaganfallpatienten. Praxis Klinische Verhaltensmedizin und Rehabilitation, 14, 288-294.

Gunzelmann, T. (1991). Problemstiuation und Beratung von Angehörigen dementiell erkrankter älterer Menschen: Stand von Forschung und Praxis. Zeitschrift für Gerontopsychologie und -psychiatrie, 4, 41-56.

Gutenbrunner, C. (2005). Rehabilitation in Deutschland. In: V. Fialka-Moser (Hrsg.), Kompendium der Physikalischen Medizin und Rehabilitation, S. 9-17, Wien: Springer. 
Gwyther (1989). Overcoming barriers: Homecare for dementia patients. Caring, 8, 1216.

Haenselt, R., Danielzik, A. \& Waack, K. (2004). Zur Evaluation von angeleiteten Gesprächsgruppen für pflegende Angehörige von Demenzkranken. Zeitschrift für Gerontologie und Geriatrie, 37, 402-410.

Häussler, B. (1996). Epidemiologie des Schlaganfalls. In: H.C. Mäurer \& C.H. Diener (Hrsg.): Der Schlaganfall, (S. 26-34). Stuttgart: Thieme.

Haley, W.E., Brown, S.L. \& Levine, E.G. (1987). Experimental evaluation of the effectiveness of group intervention for dementia caregivers. The Gerontologist, 27, 376-382.

Haley, W.E., Levine, E.G., Brown, S.L. \& Bartolucci, A.A. (1987). Stress, appraisal, coping, and social support as predictors of adaptional outcome among dementia caregivers, Psychology and Aging, 2, 323-330.

Haley, W.E., Levine, E.G., Brown, S.L., Berry, J.W. \& Hughes, G.H. (1987). Psychological, social, and health consequences of caring for a relative with senile dementia. Journal of the American Geriatrics Society, 35, 405-411.

Haley, W.E. \& Pardo, K.M. (1989). Relationship of severity of dementia to caregiving stressors, Psychology and Aging, 4, 389-392.

Halsig, N. (1998). Die psychische und soziale Situation pflegender Angehöriger: Möglichkeiten der Intervention. In: A. Kruse (Hrsg.), Psychosoziale Gerontologie, Bd. 2: Intervention, (S. 211-231). Göttingen: Hogrefe.

Hamann, G., Siebler, M. \& von Scheidt, W. (Hrsg.) (2002). Schlaganfall. Klinik, Diagnostik, Therapie. Interdisziplinäres Handbuch. München: Ecomed.

Hamilton, E.M., Braun, J.W., Kerber, P., Thurlow, C. \& Schwieterman, I. (1996). Factors associated with family caregivers' choice not to use services. American Journal of Alzheimer's Disease, July/August, 29-38.

Han, B. \& Haley, W. (1999). Family caregiving for patients with stroke. Review and analysis. Stroke, 30, 1478-1485.

Hannappel, M., Calsyn, R.J. \& Allen, G. (1993). Doees social support alleviate the depression of caregivers of dementia patients? Journal of Gerontological Social Work, 20, 35-51.

Harper, S. \& Lund, D.A. (1990). Wives, husbands, and daughters caring for institutionalized and noninstitutionalized dementia patients: Toward a model of caregiver burden. International Journal of Aging and Human Development, 30, 241-261.

Haupt, M., Karger, A., Baumgärtner, D., Kuminoti, D., Jänner, M., Schneider, F. (2000). Verbesserung von Unruhezuständen und Angst bei Demenzkranken nach psychoedukativer Gruppenarbeit mit pflegenden Angehörigen. Fortschritte in Neurologie und Psychiatrie, 68, 216-223.

Haupt, M., Siebel, U., Palm, B., Kretschmar, J.H., Jänner, M. (2000). Behandlungseffekte einer paartherapeutischen psychoedukativen Gruppenarbeit mit Demenzkranken und ihren pflegenden Angehörigen. Fortschritte in Neurologie und Psychiatrie, 68, 503-515.

Hautzinger, M. \& Bailer, M. (1993). ADS: Allgemeine Depressionsskala. Weinheim: Beltz Test Verlag.

Hearn, J., Lawler, J. \& Dowswell, G. (2003). Qualitative evaluations, combined methods and key challenges. Evaluation, 9, 30-54. 
Heier, H. (2000). Abschlussbericht für das Projekt "Aktive Beteiligung von Angehörigen geriatrischer Patienten am Rehabilitationsprozess". Unveröffentlichter Forschungsbericht. Stuttgart: Robert Bosch Stiftung.

Heinze, T. (1987). Qualitative Sozialforschung: Erfahrungen, Probleme und Perspektiven. Opladen: Westdeutscher Verlag.

Henße, S. \& Ruhnau-Wüllenweber, M. (1999). Counseling services - imperative measures in prevention. Posterbeitrag, 8. Jahrestagung der Deutschen Gesellschaft für Geriatrie, 6.-9.10., Hannover.

Hesse, S. (1994). Epidemiologie und sozialmedizinische Bedeutung des Schlaganfalls. In: K.-H. Mauritz (Hrsg.), Rehabilitation nach Schlaganfall (S. 9-14). Stuttgart: Kohlhammer.

Heuvel van den, E. Witte, L., Stewart, R., Schurre, L. Sanderman, R. \& Jong, B. (2002). Long-term effects of a group support program and an individual support program for informal caregivers of stroke patients: which caregviers benefit the most. Patient Education and Counseling, 47, 291-299.

Hofmann, W., Nikolaus, T., Pientka, L. \& Stuck, A.E. (1995). Arbeitsgruppe „Geriatrisches Assessment" (AGAST): Empfehlungen für den Einsatz von Assessment-Verfahren. Zeitschrift für Gerontologie und Geriatrie, 28, 29-34.

Holz, P. (1998). Wann nutzen pflegende Angehörige Hilfsangebote - Barrieren bei der Inanspruchnahme von Hilfen. Fachtagung Bayerisches Netzwerk PflegeAngehörigenarbeit: Handwerkszeug für die Praxis.

Hooker, K., Monahan, D., Bowman, S. R., Frazier, J.D. \& Shifren, K. (1998). Personality counts for a lot: Predictors of mental and physical health of spouse caregivers in two disease groups. Journal of Gerontology: Psychological Sciences, 53B, 73-85.

Hooker, K., Monahan, D., Shifren, K. \& Hutchinson, C. (1992). Mental and physical health of spouse caregivers: The role of personality. Psychology and Aging, 7, 367-375.

Hopf, C. (1991). Befragungsverfahren. In U. Flick (Hrsg.), Handbuch Qualitative Sozialforschung (S. 177-208). München: Psychologie Verlags Union.

Hopf, C. (2003). Qualitative Interviews - ein Überblick. In U. Flick, E. von Kardorff, I. Steinke (Hrsg.), Qualitative Forschung. Ein Handbuch (S. 349-360). Reinbek: Rowohlt.

Horowitz, A. (1985b). Sons and daughters as caregivers to older parents: Differrences in role performance and consequences. The Gerontologist, 25, 612-617.

Houlihan, J.P. (1987). Families caring for frail and demented elderly. A review of selected findings. Family Systems Medicine, 5, 344-356.

Intrieri, R.C. \& Rapp, S.R. (1994). Self-control skillfulness and caregiver burden among help-seeking elders. Journal of Gerontology, 49, 19-23.

Jackson, J.S. \& Antonucci, T.C. (1992). Social support processes in health and effective functioning of the elderly. In M.L. Wykle, E. Kahana \& J. Kowal (Eds.), Stress and health among the elderly (pp. 72-95). New York: Springer.

Jang, Y., Clay, O., Roth, D. Haley, W. \& Mittelman, M. (2004). Neuroticism and longitudinal change in caregiver depression: Impact of a spouse-caregiver intervention program. The Gerontologist, 44, 311-317.

Jansen, B. (1999). Informelle Pflege durch Angehörige, In: B. Jansen, F. Karl, H. Radebold, R. Schmitz-Scherzer (Hrsg.), Soziale Gerontologie, (S. 604-628). Weinheim: Beltz Verlag. 
Jansen, S. (2007). Fünf Jahre Alzheimer-Telefon der Deutschen Alzheimer Gesellschaft, Nervenheilkunde, 26, 685-689.

Johannsen, J. (1994). Beobachtungen und Interventionen bei Dementen und ihrem Bezugssystem aus systemischer Sicht. In: R.D. Hirsch (Hrsg.) Psychotherapie bei Demenzen. Darmstadt.

Jost, E., Voigt-Radloff, S., Hüll, M., Dykierek, P. \& Schmidtke, K. (2006). Fördergruppe für Demenzpatienten und Beratungsgruppe für Angehörige. Praktikabilität, Akzeptanz und Nutzen eines kombinierten interdisziplinären Behandlungsprogramms. Zeitschrift für Gerontopsychologie und -psychiatrie, 19, 139-150.

Kahana, E., Kahana, B. \& Kinney, J. (1990). Coping among vulnerable elders. In Z. Harel, P. Ehrlich \& R. Hubbard (Eds.). The vulnerable aged: People, services and policies (pp. 64-85). New York: Springer.

Kapur, N. (1995). External memory aids. In A.D. Baddeley, B.A. Wilson and F.N. Watts (Eds.), Memory Disorders Handbook. Chichester: Wiley.

Kardorff, E. von (1991). Qualitative Sozialforschung - Versuch einer Standortbestimmung. In U. Flick (Hrsg.), Handbuch Qualitative Sozialforschung (S. 3-10). München: Psychologie Verlags Union.

Kardorff, E. von (1999). Rehabilitation im Alter. In B. Jansen, F. Karl, H. Radebold, R. Schmitz-Scherzer (Hrsg.), Soziale Gerontologie (S. 579-603).Weinheim: Beltz.

Kardorff, E. von (2003). Qualitative Evaluationsforschung. In U. Flick, E. von Kardorff, I. Steinke (Hrsg.), Qualitative Forschung. Ein Handbuch (S. 238-250). Reinbek: Rowohlt.

Kardorff, E. von \& Schönberger, C. (2005). Angehörige in der Rehabilitation: Voraussetzungen für eine professionelle Begleitung in eine neue Realität. In C. Wendel, S. Heel, G. Lucius-Hoene \& W. Fries (Hrsg.), Zukunftswerkstatt Klinische Neuropsychologie. Therapeutische Verortungen und Visionen. Regensburg: Roderer.

Kardorff, E. von \& Stark, W. (1987). Zur Verknüpfung professioneller und alltäglicher Hilfenetze. In: Keupp, H., Röhrle, B. (Hrsg.), Soziale Netzwerke, (S. 219-244). Frankfurt/Main: Campus.

KDA - Kuratorium Deutsche Altenhilfe (Hrsg.) (1992). Verwirrte alte Menschen. Empirische Studien zur Versorgungslage und zur alltäglichen Problembewältigung durch Betroffene, Angehörige und Versorger (Forum 19). Köln.

Kelle, U. \& Erzberger, C. (2003). Qualitative Evaluationsforschung. In U. Flick, E. von Kardorff, I. Steinke (Hrsg.), Qualitative Forschung. Ein Handbuch (S. 299-309). Reinbek: Rowohlt.

Kiecolt-Glaser, J.K., Glaser, R., Shuttleworth, E.C., Dryer, C.S. Ogrocki, P. \& Speicher, C.E. (1987). Chronic stress and immunity in family caregivers of Alzheimer's Disease victims. Psychosomatic Medicine, 49, 523-535.

Killeen, M. (1990). The influence of stress and coping on family caregivers' perception of health. International Journal of Aging and Human Development, 30, 197-211.

Kim, Y., Schulz, R., \& Carver, C. S. (2007). Benefit finding in the cancer caregiving experience. Psychosomatic Medicine, 69, 283-291.

Kinney, J.M. \& Stephens, M.A. (1989). Hassles and uplifts of giving care to a family member with dementia. Psychology and Aging, 4, 402-408. 
Kinney, J.M., Stephens, M.A., Franks, M.M. \& Norris, V.K. (1995). Stresses and satisfactions of family caregivers to older stroke patients. Journal of Applied Gerontology, 14, 3-21.

Kitze, K., Gründel, I., Lehmann, A., von Cramon, D. Y. \& Wilz, G. (2002). Psychische Belastungen bei Angehörigen von Schlaganfallpatienten. Die Rehabilitation, 6, 401-407.

Kleining, G. (1982). Umriss zu einer Methodologie qualitativer Sozialforschung. Kölner Zeitschrift für Soziologie und Sozialpsychologie, 34, 224-253.

Knight, B., Lutzky, S. \& Macofsky-Urban, F. (1993). A meta-analytic review of interventions for caregiver distress. Recommendations for future research. The Gerontologist, 33, 240-248.

Knoll, J. (1993). Kurs- und Seminarmethoden: ein Trainingsbuch zur Gestaltung von Kursen und Seminaren, Arbeits- und Gesprächskreisen. Weinheim: Beltz.

Kohli, M. (1989). Das Altern der Gesellschaft: Demographische Grundlagen. Zeitschrift für Gerontopsychologie und-psychiatrie, 2, 36-41.

Kolominsky-Rabas, P., Heuschmann, P., Marschall, D., Emmert, M., Baltzer, N., Neundörfer, B., Schöffski, O. \& Krobot, K. (2006). Lifetime cost of ischemic stroke in Germany: results and national projections from a population-based stroke registry - The Erlangen Stroke Project, Stroke, 37, 1179-1183.

Kolominsky-Rabas, P., Sarti, C., Heuschmann, P., Graf, C., Siemonsen, S., Neundörfer, B., Katalinic, A., Lang, E., Gassmann, K. \& Ritter von Stockert, T. (1998). A prospective community-based study of stroke in Germany - The Erlangen Stroke Project (ESPro), Incidence and case fatality at 1, 3 and 12 months, Stroke, 29, 25012506.

Koloska, R., Rehm, J., Fichter, M. (1989). Ist die Beschwerden-Liste valide? Diagnostica, 35, 248-259.

Kosberg, J.I., Cairl, R.E. \& Keller, D.M. (1990). Components of burden: Interventive implications. The Gerontologist, 30, 236-242.

Kosloski, K. \& Montgomery, R. (1994). Investigating patterns of service use by families providing care for dependent elders. Journal of Aging \& Health, 6, 17-37.

Kotila, M., Numminen, H., Waltimo, O. \& Kaste, M. (1998). Depression after stroke: results of the FINNSTROKE study. Stroke, 29, 368-372.

Kramer, B.J. (1993a). Marital history and the prior relationship as predictors of positive and negative outcomes among wife caregivers. Family Relations, 42, 367-375.

Kramer, B.J. (1993b). Expanding the conceptualization of caregiver coping: The importance of relationship-focused coping strategies. Family Relations, 42, 383391.

Kramer, B.J. (1997a). Gain in the caregiving experience: Where are we? What next? The Gerontologist, 37, 218-232.

Kramer, B.J. (1997b). Differential predictors of strain and gain among husbands caring for wives with dementia. The Gerontologist, 37, 239-249.

Kramer, B.J. \& Kipnis, S. (1995). Eldercare and work-role conflict: Towards an understanding of gender differences in caregiver burden. The Gerontologist, 35, 340-348.

Kremer-Preiß, U. \& Zervas, E. (1994). Tagespflege in Europa: Berichte aus zehn europäischen Ländern. Köln: Kuratorium Deutsche Altershilfe.

Kruse, A. (1984). Der Schlaganfallpatient und seine Familie. Zeitschrift für Gerontologie, 17, 359-366. 
Kruse, A. (1994). Die psychische und soziale Situation pflegender Frauen - Beiträge aus empirischen Untersuchungen. Zeitschrift für Gerontologie, 27, 42-51.

Lämmler, G. (1998). Leben mit dem Schlaganfall: Belastungserleben und Bewältigungsverhalten der Frauen älterer Schlaganfallpatienten. Berlin: Köster.

Lämmler, G. (2000a). „Manchmal möcht ich streiken.“ Interviews mit Frauen älterer Schlaganfallpatienten. Psychomed, 12, 171-177.

Lämmler, G. (2000b). Zur subjektiven Belastung der Frauen älterer Schlaganfallpatienten. Zeitschrift für Neuropsychologie, 11, 107-116.

Laireiter, A. \& Lettner, K. (1993). Belastende Aspekte sozialer Netzwerke und sozialer Unterstützung: Ein Überblick über den Phänomenbereich und die Methodik. In A. Laireiter (Hrsg.), Soziales Netzwerk und soziale Unterstützung: Konzepte, Methoden und Befunde (S. 101-111). Bern: Huber.

Lambert, M.J. (2004). Bergin and Garfield's handbook of psychotherapy and behavior change $\left(5^{\text {th }}\right.$ ed.), N.Y., Wiley \& Sons.

Lamnek, S. (1995). Qualitative Sozialforschung. Band 1. Methodologie. 3. korrigierte Auflage. Weinheim: Psychologie Verlags Union.

Lamura, G., Mnich, E., Wojszel, B., Nolan, M., Krevers, B., Mestheneos, L. \& Döhner, H. (2006). Erfahrungen von pflegenden Angehörigen älterer Menschen in Europa bei der Inanspruchnahme von Unterstützungsleistungen. Zeitschrift für Gerontologie und Geriatrie, 39, 429-442.

Langhammer, B. (2007). Stroke patients and long-term training: is it worthwhile? A randomized comparison of two different training strategies after rehabilitation. Stroke, 21, 495-510.

Laux, L. (1981). Psychologische Streßkonzeptionen. In: H. Thomae (Hrsg.), Allgemeine Psychologie: Motivation. Handbuch der Psychologie, Bd. 2, 2. Auflage. Göttingen: Hogrefe.

Lawton, M.P., Brody, E.M. \& Saperstein, A.R. (1989). A controlled study of respite service for caregivers of Alzheimer's patients. The Gerontologist, 29, 8-16.

Lawton, M.P., Kleban, M.H., Moss, M., Rovine, M. \& Glicksman, A. (1989). Measuring caregiver appraisal. Journal of Gerontology, 44, 61-71.

Lazarus, R.S. (1991). Emotion and adaption. London: Oxford University Press.

Lazarus, R.S. (1993a). Coping theory and research: Past, present, and future. Psychosomatic Medicine, 55, 234-247.

Lazarus, R.S. (1993b). Why we should think of stress as a subset of emotion. In L. Goldberger \& S. Brenitz (Eds.), Handbook of Stress - Theoretical and Clinical Aspects (pp. 21-39). New York: The Free Press.

Lazarus, R.S. \& DeLongis, A. (1983). Psychological stress and coping in aging. American Psychologist, 38, 245-254.

Lazarus, R.S. \& Folkman, S. (1984). Stress, appraisal and coping. New York: Springer.

Lazarus, R.S. \& Folkman, S. (1987). Trasactional theory and research on emotions and coping. European Journal of Personality, 1, 141-170.

Lehr, U. (1991). Psychologie des Alterns. Heidelberg: Quelle und Meyer.

Leipold, B. (2004). Bewältigungsverhalten und Persönlichkeitswachstum pflegender Angehöriger. Berlin: Dissertation, Fachbereich Erziehungswissenschaft und Psychologie, Freie Universität.

Leipold, B. Schacke, C. \& Zank, S. (2005). Prädiktoren der Veränderung von Belastungen pflegender Abgehöriger: Längsschnittliche Befunde der LEANDER-

Studie. In T. Klie, A. Buhl, H. Entzian, A. Hedtke-Becker \& H. Wallrafen-Dreisow 
(Hrsg.), Die Zukunft der gesundheitlichen, sozialen und pflegerischen Versorgung älterer Menschen (S. 72-81). Frankfurt a. M.: Mabuse-Verlag.

Leipold, B., Schacke, C. \& Zank, S. (2005). Zur Veränderung der Depressivität pflegender Angehöriger: Der Beitrag von Persönlichkeitswachstum und Akzeptanz der Demenzerkrankung. In: T. Klie, A. Buhl, H. Entzian, A. Hedtke-Becker, H. Wallrafen-Dreisow (Hrsg.), Die Zukunft der gesundheitlichen, sozialen und pflegerischen Versorgung älterer Menschen (S. 34-42). Frankfurt/M.: MabuseVerlag.

Leipold, B. Schacke, C. \& Zank, S. (2006). Prädiktoren von Persönlichkeitswachstum bei pflegenden Angehörigen demenziell Erkrankter. Zeitschrift für Gerontologie und Geriatrie, 39, 227-232.

Lenzen-Großimmlinghaus, R. \& Steinhagen-Thiessen, E. (2000). Geriatrie und geriatrische Rehabilitation. In: H.-W. Wahl \& C. Tesch-Römer (Hrsg.) Angewandte Gerontologie in Schlüsselbegriffen (S. 290-295). Stuttgart: Kohlhammer.

Leys, D., Ringelstein, B., Kaste, M. \& Hacke, W. (2007). Facilities available in European hospitals treating stroke patients. Stroke, 38, 2985-2993.

Lieberman, M.A. \& Kramer, J.H. (1991). Factors affecting decisions to institutionalize demented elderly. The Gerontologist, 31, 371-374.

Livingston, M.G., Brooks, D.N. \& Bond, M.R. (1985a). Three months after severe head injury: Psychiatric and social impact on relatives. Journal of Neurology, Neurosurgery and Psychiatry, 48, 870-875.

Lomer, M. \& McLellan, D.L. (1987). Informing hospital patients and their relatives about stroke. Clinical Rehabilitation, 1, 33-37.

Low, J.T.S., Payne, S. \& Roderick, P. (1999). The impact of stroke on informal carers: a literature review. Social Science \& Medicine, 49, 711-725.

Lutz, W. (2003). Die Wiederentdeckung des Individuums in der Psychotherapieforschung. Ein Beitrag zur patientenorientierten Psychotherapieforschung und Qualitätssicherung. Tübingen: DGVT-Verlag.

Lutz, W. (2007). Evidenzbasierung in der Psychotherapie. A bridge over troubled water. Zeitschrift für Neuropsychologie, 18, 9-21.

Lutzky, S.M. \& Knight, B.G. (1994). Explaining gender differences in caregiver distress: The roles of emotional attentiveness and coping styles. Psychology and Aging, 9, 513-519.

MacNamara, S.E., Gummow, L.J., Goka, R. \& Gregg, C.H. (1990). Caregiver strain: Need for late poststroke intervention. Rehabilitation Psychology, 35, 71-78.

Magai, C., Hartung, R. \& Cohen, C.I. (1995). Caregiver distress and behavioral symptoms. In: B.A. Lawlor (Ed.): Behavioral complications in Alzheimer's disease. Washington, D.C.

Mant, J., Carter, J., Wade, D.T. \& Winner, S. (2000). Family support for stroke: a randomised controlled trial. Lancet, 256, 808-813.

Marriott, A., Donaldson, C., Tarrier, N. \& Burns, A. (2000). The effectiveness of a cognitive-behavioral intervention for reducing the burden of care in carers of patients with Alzheimer's Disease. The British Journal of Psychiatry, 176, 557562.

Mauritz, K.-H. (2004). Schlaganfall. In: G. Nelles (Hrsg.), Neurologische Rehabilitation, (S. 204-214). Stuttgart: Thieme.

Mayring, P. (1995). Qualitative Inhaltsanalyse: Grundlagen und Techniken. Weinheim: Deutscher Studien Verlag. 
Mayring, P. \& Gläser-Zikuda (2005). Die Praxis der qualitativen Inhaltsanalyse. Weinheim: Utb Verlag.

McKevitt, C., Redfern, J., Mold, F. \& Wolfe, C. (2004). Qualitative studies of stroke. A systematic review. Stroke, 35, 1499-1505.

Meier-Baumgartner, H.-P. (1998). Das Modell einer geriatrischen Fachabteilung in der Rehabilitation. In: A. Kruse (Hrsg.), Psychosoziale Gerontologie, Bd. 2: Intervention, (S. 140-152). Göttingen: Hogrefe.

Meier-Baumgartner, H.-P. et al. (1996). Empfehlungen für die geriatrische Rehabilitation. Hrsg. Bundesarbeitsgemeinschaft der geriatrischen Rehabilitationseinrichtungen e.V. Stuttgart: Fischer.

Meier-Baumgartner, H.-P. (2001). Geriatrie. Einbettung in die Versorgungslandschaft der Bundesrepublik Deutschland. Zeitschrift für Gerontologie und Geriatrie, 34, $1-9$.

Meier-Baumgartner, H.-P., Blum, H., Haas, R. \& Meins, W. (2000). Determinanten der Schlaganfall-Rehabilitation. Ergebnisse der Prospektiven Albertinen-Haus Schlaganfall-Studie (PASS). Stuttgart: Thieme.

Meier-Baumgartner, H.-P., Hain, G., Oster, P., Steinhagen-Thiessen, E. \& Vogel, W. (1998). Empfehlungen für die klinisch-geriatrische Behandlung. 2. Aufl. Jena: Gustav Fischer.

Meier-Baumgartner, H.-P., Pientka, L., Anders, J., Heer, J. \& Friedrich, C. (2002). Die Effektivität der postakuten Behandlung und Rehabilitation älterer Menschen nach einem Schlaganfall oder einer hüftgelenksnahen Fraktur. Eine evidenzbasierte Literaturübersicht des Zeitraums 1992 bis 1998. Stuttgart: Kohlhammer.

Meinders, F. (2001). Sind Angehörige von chronisch kranken älteren Menschen nur belastet? Positives Erleben und Lebenszufriedenheit bei Angehörigen demenziell erkrankter Menschen. Inaugural-Dissertation: Albert-Ludwigs-Universität Freiburg.

Meinck, M., Freigang, K., John, B., Keitel, C., Puls, E. \& Robra, B. (2002). Wohnortnahe geriatrische Rehabilitation - Zur Struktur- und Prozessqualität eines mobilen und eines ambulanten Rehabilitationsmodells. Zeitschrift für Gerontologie und Geriatrie, 35, 463-473.

Meins, W., Meier-Baumgartner, H., Neetz, D. \& Renteln-Kruse, R. von (2002). Predictors of favorable outcome in elderly stroke patients two years after discharge from geriatric rehabilitation. Zeitschrift für Gerontologie und Geriatrie, 34, 395400.

Meister, S. \& Zehle, H. (2003). Intervention bei Angehörigen demenziell Erkrankter: Wirkung der gerontopsychiatrischen Tagespflege auf die pflegebedingte Belastung. Unveröffentlichte Diplomarbeit: Freie Universität Berlin.

Miller, B.C. (1986). Family research methods. Beverly Hills, CA: Sage.

Miller, B. (1987). Gender and control among spouses of the cognitively impaired: A research note. The Gerontologist, 27, 447-453.

Miller, B. (1990). Gender differences in spouse caregiver strain: Socialization and role explanations. Journal of Marriage and the Family, 52, 311-321.

Miller, B. \& Cafasso, L. (1992). Gender differences in caregiving: Fact or artefact. The Gerontologist, 32, 498-507.

Milne, D, Pitt, I. \& Sabin, N. (1993). Evaluation of a carer support scheme for elderly people: The importance of coping. British Journal of Social Work, 23, 157-168.

Mittelman, M., Ferris, S., Shulman, E., Steinberg, G. \& Levin, B.A. (1996). A family intervention to delay nursing home placement of patients with Alzheimer disease: A 
randomized controlled trial. Journal of the American Medical Association, 276, $1725-1731$.

Mittelman, M., Roth, D., Clay, O. \& Haley, W. (2007). Preserving health of Alzheimer caregivers: Impact of a spouse caregiver intervention, American Journal of Geriatric Psychiatry, 15, 780-789.

Mittelman, M., Roth, D., Haley, W. \& Zarit, S. (2004). Effects of a caregiver intervention on negative caregiver appraisals of behavior problems in patients with Alzheimer's disease: results of a randomized trial. Journals of Gerontology, Psychological Sciences, 59, 27-34.

Mohide, E.A., Pringle, D.M., Streiner, D.L., Gilbert, J.R., Muir, G. \& Tew, M. (1990). A randomized trial of family caregiver support in the home management of dementia. Journal of the American Geriatrics Society, 38, 446-454.

Montgomery, R.J. \& Borgatta, E.F. (1989). The effects of alternative support strategies on family caregiving. The Gerontologist, 29, 457-464.

Montgomery, R.J.V. \& Kosloski, K. (1995). Respite revisited: Re-assessing the impact. In P.R. Katz, R.L. Kang \& M.D. Mezey (Eds.), Quality care in geriatric settings (pp. 47-67). New York: Springer.

Moriarty, J. (1999). Use of community and long-term care by people with dementia in the UK: A review of some issues in service provision and care and user preferences. Aging and Mental Health, 3, 311-319.

Moritz, D.J., Kasl, S.V. \& Berkman, L.F. (1989). The health impact of living with a cognitively impaired elderly spouse: Depressive symptoms and social functioning. Journal of Gerontology: Social Sciences , 44, 517-527.

Moritz, D.J., Kasl, S.V. \& Ostfeld, A.M. (1992). The health impact of living with a cognitively impaired elderly spouse: Blood pressure, self-rated health, and health behaviors. Journal of Aging and Health, 4, 244-267.

Motenko, A. (1989). The frustrations, gratifications, and well-being of dementia caregivers. The Gerontologist, 29, 166-172.

Müller, K.R. (Hrsg.) (1989). Kurs- und Seminargestaltung. Ein Handbuch für Dozentinnen und Kursleiter. München: Hueber.

Nehen, H. G. (1998). Rehabilitation in der Geriatrie aus Sicht verschiedener Disziplinen. In: A. Kruse (Hrsg.), Psychosoziale Gerontologie, Bd. 2: Intervention, (S. 127-139). Göttingen: Hogrefe.

Nelles, G. (2004). Funktionsrückbildung und neuronale Plastizität. In: G. Nelles (Hrsg.), Neurologische Rehabilitation, S. 1-13. Stuttgart: Thieme.

Nolan, M., Ingram, P. \& Watson, R. (2002). Working with family carers of people with dementia: „negotiated“ coping as an essential outcome. Dementia, 1, 75-93.

Nolan, M., Ryan, T., Enderby, P. \& Reid, D. (2002). Towards a more inclusive vision of dementia care practice and research. Dementia, 1, 193-211.

Orgass, B. (1982). Token Test (TT). Deutsche Bearbeitung des Token Tests von E. De Renzi \& L.A. Vignolo. Weinheim: Beltz.

Ory, M.G., Hoffman, R.R., Yee, J.L., Tennstedt, S. \& Schulz, R. (1999). Prevalence and impact of caregiving: A detailed comparison between dementia and non-dementia caregivers. The Gerontologist, 39, 177-185.

Ostwald, S.K., Hepburn, K.W., Caron, W., Burns, T. \& Mantell, R. (1999). Reducing caregiver burden: A randomized psycho-educational intervention for caregivers of persons with dementia. The Gerontologist, 39, 299-309. 
Pagel, M.D., Erdly, W.W. \& Becker, J. (1987). Social networks: We get by with (and in spite of) a little help from our friends. Journal of Personality and Social Psychology, 4, 793-804.

Pain, H. \& McLellan, D.L. (1990). The use of individualised booklets after stroke. Clinical Rehabilitation, 4, 265-272.

Parker, G. (1997). Coping with caring for a person with dementia. In S. Hunter (Ed.). Dementia - Challenges and new directions. London: Jessica Kingsley.

Pearlin, L.I., Aneshensel, C.S., Mullan, J.T. \& Whitlatch, C.J. (1996). Caregiving and its social support. In R.H. Binstock \& L.K.George (Eds.), Handbook of Aging and the Social Sciences (pp. 283-302). California: Academic Press.

Pearlin, L.I., Lieberman, M.A., Menaghan, E.G. \& Mullan, J.T. (1981). The stress process. Journal of Health and Social Behavior, 22, 337-356.

Pearlin, L.I., Mullan, J.T., Semple, S.J. \& Skaff, M.M. (1990). Caregiving and the stress process: An overview of concepts and their measures. The Gerontologist, 30, 583591.

Pearlin, L.I., Turner, H. \& Semple, S. (1989). Coping and the mediation of caregiver stress. In: E. Light \& B.D. Leibowitz (Eds.), Alzheimer's disease treatment and stress: directions in research (pp. 198-217). Maryland: National Institute of Mental Health.

Pedlar, D. \& Biegel, D. (1999). The impact of family caregiver attitudes on the use of community services for dementia care. The Journal of Applied Gerontology, 18, 201-221.

Peters-Davis, N.D., Moss, M.S. \& Pruchno, R.A. (1999). Children-in-law in caregiving families. The Gerontologist, 39, 66-75.

Pickett, T., Altmaier, E. \& Paulsen, J. (2007). Caregiver burden in Huntington's disease. Rehabilitation Psychology, 52, 311-318.

Pillemer, K. \& Suitor, J.J. (1996). "It takes one to help one": Effects of similar others on the well-being of caregivers. Journal of Gerontology: Social Sciences, 51, S250S257.

Pinquart, M. \& Sörensen, S. (2003a). Differences between caregivers and noncaregivers in psychological health and physical health: A meta-analysis. Psychology and Aging, 18, 250-267.

Pinquart, M. \& Sörensen, S. (2005). Ethnic differences in stressors, resources, and psychological outcomes of family caregiving: A meta-analysis. The Gerontologist, 45, 90-106.

Pope, C. \& Mays, N. (1995). Reaching the parts other methods cannot reach: an introduction to qualitative methods in health and health services research. British Medical Journal, 311, 42-45.

Powers, D., Gallagher-Thompson, D. \& Kraemer, H. (2002). Coping and depression in Alzheimer's caregivers. Longitudinal evidence of stability. Journal of Gerontology, Psychological Sciences, 57, 205-211.

Pratt, C.C., Schmall, V.L., Wright, S. \& Cleland, M. (1985). Family caregivers and dementia. Social Casework, 67, 119-124.

Pruchno, R. (2003). Enmeshed lives: Adult children with developmental disabilities and their aging mothers. Psychology and Aging, 18, 851-857.

Pruchno, R. \& Resch, N.L. (1989). Husbands and wives as caregivers: Antecedents of depression and burden. The Gerontologist, 29, 159-165. 
Pusey, H. \& Richards, D. (2001). A systematic review of the effectiveness of psychosocial interventions for carers of people with dementia. Aging \& Mental Health, 5, 107-119.

Quayhagen, M.P. \& Quayhagen, M. (1988). Alzheimer's stress: Coping with the caregiving role. The Gerontologist, 28, 391-396.

Quayhagen, M.P. \& Quayhagen, M. (1989). Differential effects of family-based strategies on Alzheimer'sdisease. The Gerontologist, 29, 150-155.

Qureshi, H., Bamford, C., Nicholas, E., Patmore, C. \& Harris, J. C. (2000). Outcomes in social care practice: Developing an outcome focus in care mangagement and use surveys. York: Social Policy Research Unit, University of York.

Rabins, P.V., Mace, N. \& Lucas, M.J. (1982). The impact of dementia on the family. Journal of the American Medical Association, 248, 333-335.

Radloff, L.S. (1977). The CES-D scale: A self-report depression scale for research in the general population. Applied Psychological Measurement, 3, 385-401.

Rakowski, W. \& Clark, N.M. (1985). Future outlook, caregiving, and care-receiving in the family context. The Gerontologist, 25, 628-633.

Rank, M. (1992). The blending of qualitative and quantitative methods in understanding childbearing among welfare recipients. In J.F. Gilgun, K. Daly \& G. Handel (Hrsg.), Qualitative methods in family research, (S. 281-300). Newbury Park, CA: Sage.

Rapp, S.R., Parisi, S.A., Walsh, D.A. \& Wallace, C.E. (1988). Detecting depression in elderly medical inpatients. Journal of Consulting and Clinical Psychology, 56, 509513.

Rieckmann, N. (2003). Adaptation and resilience in middle-aged, young old, and old cataract patients. Dissertationsschrift. Berlin: Freie Universität.

Rivera, P.A., Rose, J.M., Futterman, A., Lovett, S.B. \& Gallagher-Thompson, D. (1991). Dimensions of perceived social support in clinically depressed and nondepressed female caregivers. Psychology and Aging, 6, 232-237.

Robinson, L., Francis, J., James, P., Tindle, N., Greenwell, K. \& Rodgers, H. (2005). Caring for carers of people with stroke: developing a complex intervention following the Medical Research Council framework. Clinical Rehabilitation, 19, 560-571.

Robinson, R.G., Starr, L.B., Kubos, K.L. \& Price, T.R. (1983). A two-year longitudinal study of post-stroke mood disorders: Findings during the initial evaluation. Stroke, 14, 736-741.

Rodgers, H., Bond, S. \& Curless, R. (2001). Inadequacies in the provision of information to stroke patients and their families. Age and Ageing, 30, 129-133.

Rößler, D. (1995). Psychische Korrelate bei der Pflege von Angehörigen. Unveröffentlichte Diplomarbeit der Phillips-Universität Marburg.

Rolland, J. (2006). Resilienz von Familien mit kranken und behinderten Angehörigen: Ein integratives Modell. In: R. Welter-Enderlin \& B. Hildenbrand, Resilienz Gedeihen trotz widriger Umstände, (S. 158-191). Heidelberg: Carl-Auer-Verlag.

Rose-Rego, S.K., Strauss, M.E. \& Smyth, K.A. (1998). Differences in the perceived well-being of wives and husbands caring for persons with Alzheimer's disease. The Gerontologist, 38, 224-230.

Rosenthal, M. \& Young, T. (1988). Effective family intervention after brain injury: theory and practice. Journal of Head Trauma Rehabilitation, 3, 42-50. 
Roskies, E. \& Lazarus, R.S. (1980). Coping theory and teaching of coping skills. In: P. Davidson (Ed.). Behavioral medicine: changing health life styles. New York: Brunner/Mazel.

Rossi, P.H. \& Freeman, H.E. (1993). Evaluation: A systematic approach. Newbury Park, CA: Sage.

Rückert, W. (1999). Demographie. In: B. Jansen, F. Karl, H. Radebold \& R. SchmitzScherzer (Hrsg.), Soziale Gerontologie, (S. 142-154). Weinheim: Beltz Verlag.

Rusch, K., Frommelt, P. \& Thun, Th. (1992). Modell der Angehörigenbetreuung in der neurologischen Rehabilitation. In: K.-H. Mauritz \& V. Hömberg (Hrsg.), Neurologische Rehabilitation, (S. 224-227). Bern: Huber.

Rutter, M. (1999). Resilience concepts and findings: implications for family therapy. Journal of Family Therapy, 21, 119-144.

Sachverständigenrat für die Konzertierte Aktion im Gesundheitswesen (2001). Über-, Unter- und Fehlversorgung, Bd. III. Baden-Baden: Nomos.

Sachverständigenrat zur Begutachtung der Entwicklung im Gesundheitswesen (2005). Koordination und Qualität im Gesundheitswesen. Baden-Baden: Nomos.

Sarason, I.G. \& Sarason, B.R. (Eds.) (1985). Social support: Theory, research and applications. Dordrecht, The Netherlands: Martinus Nijhoff.

Schacke, C. (2001). Die Entlastungsfunktion gerontopsychiatrischer Tagesstätten für die pflegenden Angehörigen der Besucher. Berlin: Mensch-und-Buch-Verlag.

Schacke, C. (2006). Gewalt in engen persönlichen Beziehungen älterer Pflegebedürftiger. In: T.Görgen \& B. Nägele (Hrsg.), Wehrlos im Alter - Strategien gegen Gewalt in engen persönlichen Beziehungen älterer Menschen, (S. 34-42).

KFN Materialien für die Praxis

Schacke, C. \& Zank, S. (2004). Interventionskonzepte. In: A. Kruse \& M. Martin (Hrsg.). Enzyklopädie der Gerontologie: Alternsprozesse in multidisziplinärer Sicht, (S. 419-436). Bern: Huber.

Schacke, C. \& Zank, S. (2006). Measuring the Effectiveness of Adult Day Care as a Facility to Support Family Caregivers of Dementia Patients. Journal of Applied Gerontology, 25, 65-81.

Scharlach, A.E. (1987). Relieving feelings of strain among women with elderly mothers. Psychology and Aging, 2, 9-13.

Schmall, V.L. (1995). Family Caregiver Education and Training: Enhancing SelfEfficiacy. Journal of Case Management, 4, 156-162.

Schmidt, C. (2003). Analyse von Leitfadeninterviews. In U. Flick, E. von Kardorff, I. Steinke (Hrsg.), Qualitative Forschung. Ein Handbuch (S. 447-456). Reinbek: Rowohlt.

Schneekloth, U. (2006). Entwicklungstrends und Perspektiven in der häuslichen Pflege. Zeitschrift für Gerontologie und Geriatrie, 39, 405-412.

Schneekloth, U. \& Leven, I. (2002). Hilfe- und Pflegebedürftige in Privathaushaltne in Deutschland: Schnellbereicht. München: Infratest Sozialforschung.

Schneekloth, U., Potthoff, P., Piekara, R. \& V. Rosenbladt, B. (1996). Hilfe- und Pflegebedürftige in privaten Haushalten. Band 111.2, Schriftenreihe des Bundesministeriums für Familie, Senioren, Frauen und Jugend. Stuttgart: Kohlhammer. 
Schönberger, C. \& Kardorff, E.v. (2003). Angehörige Krebskranker in der Rehabilitation - ihre Leistungen und ihre Entlastungsbedarf. Ausgewählte Ergebnisse einer Pilotstudie. Praxis Klinische Verhaltensmedizin und Rehabilitation, 62, 130-137.

Schönberger, C. \& Kardorff, E.v. (2004). Mit dem kranken Partner leben. Anforderungen, Belastungen und Leistungen von Angehörigen Krebskranker. Soziologische Fallstudien. Opladen: Leske und Budrich.

Schreiner, A. S., Morimoto, T., Kakuma, T., Arai, Y. \& Zarit, S. H. (2006). Assessing family caregivers' mental health using a statistically derived cutoff score for the Zarit Burden Interview. Aging and Mental Health, 10, 107-111.

Schütz, R.-M. (1994). Der Schlaganfall. In R.-M. Schütz \& H.P. Meier-Baumgartner (Hrsg.), Der Schlaganfallpatient (S. 173-191). Bern: Huber.

Schulz, R. (Ed.) (2000). Handbook on dementia caregiving: Evidence-based interventions for family caregivers. New York: Springer.

Schulz, R., O’Brien, A.T., Bookwala, J. \& Fleissner, K. (1995). Psychiatric and physical morbidity effects of dementia caregiving: Prevalence, correlates, and causes. The Gerontologist, 35, 771-791.

Schulz, R., O’Brien, A., Czaja, S.J., Ory, M., Norris, R., Martire, L.M., Belle, S.H., Burgio, L., Gitlin, L., Coon, D., Burns, R., Gallagher-Thompson, D. \& Stevens, A. (2002). Dementia caregiver intervention research: In search of clinical significance. The Gerontologist, 42, 589-602.

Schulz, R., Tompkins, C. \& Rau, M.T. (1988). A longitudinal study of the psychosocial impact of stroke on primary support persons. Psychology and Aging, 3, 131-141.

Schulz, R. \& Williamson, G.M. (1991). A 2-year longitudinal study of depression among Alzheimer's caregivers. Psychology and Aging, 6, 569-578.

Schwarzer, R. (1993). Streß, Angst und Handlungsregulation. Stuttgart: Kohlhammer.

Seitz, R.J. \& Bütefisch, C.M. (2006). Recovery from ischemic stroke: a translational research perspective for neurology. Future Neurology, 1, 571-586.

Serrano, S., Domingo, J., Rodriguez-Garcia, E., Castro, M. \& del Ser, T. (2007). Frequency of cognitive impairment without dementia in patients with stroke. A twoyear follow-up study. Stroke, 38, 105-110.

Shurgot, G. \& Knight, B. (2005). Influence of neuroticism, ethnicity, familism, and social support on perceived burden in dementia caregivers: pilot test of the transactional stress and social support model. Journal of Gerontology, Psychological Sciences, 60, 331-334.

Sistler, A. (1989). Adaptive coping of older caregiving spouses. Social Work, 415-420.

Sloan, M.A., Kittner, S.J., Rigamont, D. \& Price, T.R. (1991). Occurence of stroke associated with use/abuse of drugs. Neurology, 41, 1358-1364.

Smith, J., Forster, A. \& Young, J. (2004). A randomized trial to evaluate an education programme for patients and carers after stroke. Clinical Rehabilitation, 18, 726-736.

Socialdata (1980). Anzahl und Situation zu Hause lebender Pflegebedürftiger. Stuttgart: Kohlhammer.

Sörensen, S., Pinquardt, M. \& Duberstein, P. (2002). How effective are interventions with caregivers? An updated meta-analysis. The Gerontologist, 42, 356-372.

Spaid, W.M. \& Barusch, A.S. (1994). Emotional closeness and caregiver burden in the marital relationship. Journal of Gerontological Social Work, 21, 197-211. 
Stein, P.N., Gordon, W.A., Hibbard, M.R. \& Sliwinski, M.J. (1992). An examination of depression in the spouses of stroke patients. Rehabilitation Psychology, 37, 121129.

Steinhagen-Thiessen, E. \& Borchelt, M. (1996). Morbidität, Medikation und Funktionalität im Alter. In: K.U. Mayer \& P.B. Baltes (Hrsg.) Die Berliner Altersstudie, (S. 151-183). Berlin: Akademie Verlag.

Steinhagen-Thiessen, E., Gerok, W. \& Borchelt, M. (1992). Innere Medizin und Geriatrie. In P.B. Baltes \& J. Mittelstraß (Hrsg.), Zukunft des Alterns und gesellschaftliche Entwicklung, (S. 124-150). Berlin: De Gruyter.

Steinhagen-Thiessen, E., Hamel, G., Lüttje, D., Oster, P., Plate, A. \& Vogel, W. (2003). Geriatrie - quo vadis? Zeitschrift für Gerontologie und Geriatrie, 366-377.

Sterker, M. (1998). Das Schlaganfall-Informationstelefon - Ein Beitrag zur Senkung des Informationsdefizits in Prävention, Therapie und Rehabilitation des Schlaganfalls. Neurologische Rehabilitation, 4, 48-51.

Stone, R., Cafferata, G.L. \& Sangl, J. (1987). Caregivers of the frail elderly: a national profile. The Gerontologist, 27, 616-626.

Stroke Unit Triallists' Collaboration (1997). How do stroke units improve patients outcomes? A collaborative systematic review of the randomized trials. Stroke, 28, 2139-2144.

Stull, D.E., Kosloski, K. \& Kercher, K. (1994). Caregiver burden and generic wellbeing: Opposite sides of the same coin? The Gerontologist, 34, 88-94.

Suitor, J. \& Pillemer, K. (1996). Sources of support and interpersonal stress in the networks of married caregiving daughters: Findings from a 2-year longitudinal study. Journal of Gerontology, 51, S297-306.

Thiede Call, C., Finch, M., Huck, S. \& Kane, R. (1999). Caregiver burden from social exchange perspective: Caring for older people after hospital discharge. Journal of Marriage and the Family, 61, 668-699.

Thoma, J., Schacke, C. \& Zank, S. (2004). Gewalt gegen demenziell Erkrankte in der Familie: Datenerhebung in einem schwer zugänglichen Forschungsgebiet. Zeitschrift für Gerontologie und Geriatrie, 37, 349-350.

Thommessen, B., Aarsland, D., Brackhus, A., Oksengaard, A., Engedal, K. \& Laake, K. (2002). The psychosocial burden on spouses of the elderly with stroke, dementia, and Parkinson's disease. International Journal of Geriatric Psychiatry, 17, 78-84.

Thompson, C. \& Briggs, M. (2000). Support for carers of people with Alzheimer's type dementia (Issue 4). Oxford: Cochrane Review.

Thomson, E.H., Futterman, A.M., Gallagher-Thomson, D., Rose, J.M. \& Lovett, S.B. (1993). Social support and caregiving burden in family caregivers of frail elders. Journal of Gerontology, 48, 245-254.

Toseland, R. \& Rossiter, C.M. (1989). Group interventions to support family caregivers: A review and analysis. The Gerontologist, 29, 438-448.

Toseland, R., Rossiter, C.M. \& Labrecque, M. (1989a). The effectiveness of peer-led and professionally-led groups to support family caregivers, The Gerontologist, 29, 465-471.

Toseland, R. \& Smith, G. (1990). Effectiveness of individual counseling by professional and peer helpers for family caregiving of the elderly. Psychology and Aging, 5, 256263. 
Townsend, A., Noelker, L., Deimling, G. \& Bass, D. (1989). Longitudinal impact of interhousehold caregiving on adult children's mental health. Psychology and Aging, 4, 393-401.

Tyman, R.V. (1994). The stress experienced by caregivers of stroke survivors: Is it all in the mind, or is it also in the body? Clinical Rehabilitation, 8, 341-345.

Upton, A.R.M. \& Finlayson, M.A.J. (1987). Behavioral and organic mental changes in strike. In: M.E. Brandstater, J.V. Basmajian (eds.): Stroke rehabilitation, (pp. 318329). Baltimore: Williams Wilkins Co.

Vitaliano, P., Russo, J., Young, H.M. \& Teri, L. \& Maiuro, R.D. (1991). Predictors of burden in spouse caregivers of individuals with Alzheimer's Disease. Psychology and Aging, 6, 392-402.

Vitaliano, P., Zhang, J. \& Scanlan, J. (2003). Is caregiving hazardous to one's physical health? A meta analysis. Psychological Bulletin, 129, 946-972.

Wade, D. (1993). Stroke. In R. Greenwood, M.P. Barnes, T.M. McMillan \& C.D. Ward (Eds.), Neurological Rehabilitation (pp. 451-458). Edinburgh: Churchill Livingstone.

Wade, D.T. \& Hewer, L.R. (1987b). Functional abilities after stroke: measurement, natural history and prognosis. Journal of Neurology, Neurosurgery and Psychiatry, 50, 177-182.

Wagner, B. (2004). Geriatrische Versorgung in Deutschland: Versorgungsketten zwischen Krankenhaus, Rehabilitation und Pflege? Hamburg: Kovac.

Walker, R.J., Pomeroy, E.C., McNeil, J.S. \& Franklin, C. (1994). A psychoeducational model for caregivers of patients with Alzheimer's disease. Journal of Gerontological Social Work, 22, 75-91.

Wallhagen, M. (1992). Caregiving demands: Their difficulty and effects on the wellbeing of elderly caregivers. Scholarly Inquiry for Nursing Practice, 6, 111-127.

Walz, T.H. \& Brown, P. (1991). Family based long-term care for the elderly. Stress considerations. In: M. Wyhle (Ed.), Stress and health among the elderly, (pp. 223239). New York: Springer.

Weber, H. (1990). Das Tagebuch in der Belastung- und Bewältigungsforschung. Memorandum Nr. 8, Lehrstuhl Psychologie IV, Universität Bamberg.

Weiller, C. (1996). Ätiologie und Pathogenese des Schlaganfalls. In: H.C. Mäurer \& C.H. Diener (Hrsg.): Der Schlaganfall, (S. 1-25). Stuttgart: Thieme.

Welter-Enderlin, R. \& Hildenbrand, B. (2006). Resilienz - Gedeihen trotz widriger Umstände. Heidelberg: Carl-Auer-Verlag.

Wendel, C. (2005). Kontextualisierung und Rückversicherung. Über die Arbeit mit Angehörigen in de Neuropsychologie. In C. Wendel, S. Heel, G. Lucius-Hoene \& W. Fries (Hrsg.), Zukunftswerkstatt Klinische Neuropsychologie. Therapeutische Verortungen und Visionen. Regensburg: Roderer.

Wentura, D., Greve, W. \& Klauer, T. (2002). Theorien der Bewältigung. In D. Frey \& M. Irle (Hrsg.), Theorien der Sozialpsychologie: Band III, Motivations-, Selbst- und Informationsverarbeitsungstheorien, (S. 101-125). Bern: Huber.

WHO (2001). International Classification of Functioning, Disability and Health (ICF). www.who.int/classifications/icf/site/icftemplate/cfm.

Williamson, G.M. \& Schulz, R. (1990). Relationship orientation, quality of prior relationship, and distress among caregivers of Alzheimer's patients. Psychology and Aging, 5, 502-509. 
Wilson, B.A., Emslie, H.C., Quirk, K. \& Evans, J.J. (2001). Reducing everyday memory and planning problems by means of a paging system: a randomised control crossover study. Journal of Neurology, Neurosurgery and Psychiatry, 70, 477-482.

Wilson, T.P. (1982). Qualitative „oder“ quantitative Methoden in der Sozialforschung. Kölner Zeitschrift für Soziologie und Sozialpsychologie, 34, 487-508.

Wilz, G. (2002). Belastungsverarbeitung bei pflegenden Angehörigen von Demenzkranken. Eine Tagebuchstudie. Göttingen: Hogrefe.

Wilz, G., Gunzelmann, T., Adler, C., Brähler, E. (1998). Gruppenprogramm für pflegende Angehörige von Demenzkranken. Zeitschrift für Gerontopsychologie und -psychiatrie, 11, 97-106.

Wilz, G., Adler, C. \& Gunzelmann, T. (2001). Gruppenarbeit bei pflegenden Angehörigen von Demenzkranken. Ein therapeutischer Leitfaden. Göttingen: Hogrefe.

Wilz, G. , Kalytta, T. \& Küssner, C. (2005). Quantitative und qualitative Diagnostik von Belastungen und Belastungsverarbeitung bei pflegenden Angehörigen. Zeitschrift für Gerontologie und -psychiatrie, 18, 259-277.

Wright, S.D., Lund, D.A., Caserta, M.S. \& Pratt, C. (1991). Coping and caregiver wellbeing: The impact of maladaptive strategies. Journal of Gerontological Social Work, 17, 75-91.

Yesavage, J.A., Brink, T.L., Rose, T.L., Lum, O., Huang, V., Adey, M. \& Leirer, O. von (1983). Development and validation of a geriatric depression screening scale: A preliminary report. Journal of Psychiatric Research, 17, 37-49.

Young, R.F. \& Kahana, E. (1989). Specifiying caregiver outcomes: Gender and relationship aspects of caregiving strain. The Gerontologist, 29, 660-666.

Zank, S. \& Schacke, C. (2007). Abschlußbericht der Phase 2 der „Längsschnittstudie zur Angehörigenbelastung durch die Pflege demenziell Erkrankter" (LEANDER). Bundesministerium für Familie, Senioren, Frauen und Jugend.

Zank, S., Schacke, C. \& Leipold, B. (2006). Berliner Inventar zur Angehörigenbelastung - Demenz (BIZA-D). Zeitschrift für Klinische Psychologie und Psychotherapie, 35, 296-305.

Zarit, S.H. (1989). Do we need another "stress and caregiving" study? The Gerontologist, 29, 147-148.

Zarit, S.H. (1992). Measures in family caregiving research. In B. Bauer (Ed.), Conceptual and methodological issues in family caregiving research. Proceedings of the invitational conference on family caregiving research (S. 1-19). Toronto: University of Toronto.

Zarit, S.H., Anthony, C.R. \& Boutselis, M. (1987). Interventions with caregivers of dementia patients: Comparison of two approaches. Psychology and Aging, 2, 225232.

Zarit, S.H., Gaugler, J.E. \& Jarrott, S.E. (1999). Useful services for families: Research findings and directions. International Journal of Geriatric Psychiatry, 14, 165-177.

Zarit, S.H. \& Leitsch, S.A. (2001). Developing and evaluating community based intervention programs for Alzheimer's patients and their caregivers. Aging \& Mental Health, 5, S84-S98.

Zarit, S.H., Reever, K.E. \& Bach-Peterson, J. (1980). Relatives of the impaired elderly: Correlates of feelings of burden. The Gerontologist, 20, 649-655. 
Zarit, S.H., Stephens, M.A., Townsend, A. \& Greene, R. (1998). Stress reduction for family caregivers: effects of adult day care use. Journals of Gerontology, 53B, S267-S277.

Zarit, S.H., Stephens, M.A. Townsend, A., Greene, R. \& Femia, E. (2003). Commentary on "Evaluation of geriatric day care units: effexts on patients and caregivers" and authors' reply. Journal of Gerontology, Psychological Sciences, 58B, 195-199.

Zarit, S.H., Todd, P.A. \& Zarit, J.M. (1986). Subjective burden of husbands and wives as caregivers. A longitudinal study. The Gerontologist, 26, 260-266.

Zarit, S.H. \& Zarit, J.M. (1983). The memory and behaviour problems checklist and the burden interview. Pennstate University: Gerontology Center.

Zerssen, D.v. (1976). Beschwerdeliste. Belz Test Gesellschaft mbH, Weinheim.

Zung, W. (1965). A self rating depression scale. Archives of General Psychiatry, 12, 6370.

Zung, W. (1971a). A rating instrument for anxiety disorders. Psychosomatics, 12, 371379.

Zung, W. (1971b). The differentiation of anxiety and depressive disorders. Psychosomatics, 12, 380-384.

Zung, W. (1976). Self-Rating Anxiety Scale. In: W. Guy (Ed.), ECDEU Assessment Manual for Psychopharmacology, (pp. 337-340). Rockville, Maryland: Rev. Ed. 


\title{
Anhang A: Materialien für die quantitative Untersuchung
}

\author{
Forschungsgruppe Geriatrie am Ev. Geriatriezentrum Berlin \\ Medizinische Fakultät der Humboldt Universität zu Berlin
}

Ärztliche Leitung: Prof. Dr. med. E. Steinhagen-Thiessen

Reinickendorfer Str. 61, 13347 Berlin

Tel. 030/450-53127

Liebe Angehörige,

unser Angehörigenkurs findet im Rahmen eines Modellprojekts statt und wird wissenschaftlich begleitet (Leitung: Frau Prof. Dr. med. E. Steinhagen-Thiessen).

Dabei interessiert uns, wie Angehörige von Schlaganfall-Patienten ihre Situation bewältigen.

Insgesamt werden Sie 3 Fragebögen zum Ausfüllen erhalten: Der erste liegt Ihnen heute vor. Den zweiten und dritten schicken wir Ihnen 2-3 Monate, bzw. 8-9 Monate nach Ende des Angehörigenkurses mit einem frankierten Rückumschlag zu.

Mit Ihrer Teilnahme an der wissenschaftlichen Begleitstudie helfen Sie uns, wichtige Erkenntnisse über Ihre Situation zu gewinnen. Damit möchten wir unser Angebot für die Patienten und ihre Angehörigen weiter verbessern.

Die Teilnahme am Angehörigenkurs verpflichtet Sie aber nicht zur Teilnahme an der wissenschaftlichen Begleitstudie. Sie können selbstverständlich auch am Kurs teilnehmen, ohne die Fragebögen auszufüllen.

Bei der Auswertung der Fragebögen wird der Datenschutz sorgfältig beachtet. Da Sie den Fragebogen ohne Angabe Ihres Namens ausfüllen, können Ihre Daten nicht Ihrer Person zugeordnet werden.

Falls Sie Fragen zum Ausfüllen des Fragebogens oder zur Begleitstudie im allgemeinen haben, wenden Sie sich bitte persönlich oder telefonisch an mich (Frau Heier, Tel. 45053 127). Ich beantworte Ihnen gerne alle Fragen.

Damit Ihre Antworten anonym bleiben und wir die 3 Fragebögen, die Sie insgesamt ausfüllen, einander zuordnen können, bitten wir Sie, eine Codenummer einzutragen:

\section{Code-Nummer}

Monat Ihrer Geburt in Ziffern (z.B. April $=04$ )

1. Buchstabe des Vornamens Ihrer Mutter (z.B. Anneliese = A)

1. Buchstabe des Vornamens Ihres Vaters (z.B. Gerd = G) 


\section{Hinweise zum Ausfüllen des Fragebogens}

Der Fragebogen besteht aus mehreren Abschnitten. Alle Fragebogenabschnitte werden zu Beginn kurz erläutert. Für die Auswertung ist es wichtig, daß Sie den Fragebogen in der vorgegebenen Reihenfolge ausfüllen.

In der Regel sind den einzelnen Aussagen oder Fragen Antwortkästchen zugeordnet. Bitte kreuzen Sie das jeweils für Sie zutreffende Kästchen an. Es gibt dabei keine "richtigen" oder "falschen" Antworten. Es kommt allein auf Ihre persönliche Einschätzung an.

\section{Beispiel}

\begin{tabular}{|lcccc|}
\hline Während der letzten Woche konnte ich & $\begin{array}{c}\text { stimmt } \\
\text { nicht }\end{array}$ & $\begin{array}{c}\text { stimmt } \\
\text { kaum }\end{array}$ & $\begin{array}{c}\text { stimmt } \\
\text { eher }\end{array}$ & $\begin{array}{c}\text { stimmt } \\
\text { genau }\end{array}$ \\
schlecht schlafen...................................... & $\square$ & $\square$ & $\square$ & $\square$ \\
\hline
\end{tabular}

Die Beantwortung des Fragebogens wird etwa 45 Minuten dauern.

Bitte beantworten Sie die Fragen so sorgfältig wie möglich und lassen Sie möglichst keine Fragen aus!

Überlegen Sie beim Ausfüllen nicht zu lange, denn oft ist der erste Gedanke, der Ihnen dazu einfällt, der zutreffendste.

Schon im Voraus vielen Dank für Ihre Mühe! 
Zunächst interessieren uns einige Angaben zu Ihrer Person und zu Ihrem/Ihrer Angehörigen, die sich möglicherweise seit dem Ausfüllen des ersten Fragebogens verändert haben. Bitte kreuzen Sie das jeweils auf Sie zureffende Kästchen an, oder tragen Sie die entsprechenden Antworten ein.

1. Sind Sie zur Zeit berufstätig?

nein

$\square$ ja, und zwar Studen in der Woche als

2. Lebt Ihr Angehöriger in Ihrem Haushalt?

nein

$\square$ ja

3. An wieviel Tagen in der Woche swird Ihre Angehöriger durchschnittlcih von Ihnen versorgt?

An __ Tag(en) pro Woche

4. Welche der Versorgungstätigkeiten und Hilfeleistungen führen Sie aus?

(Mehrfachnennungen möglich)

$\square$ Waschen/Duschen

$\square$ Rasieren/Frisieren/Nagelpflege

An- bzw. Ausziehen

Hilfe bei Toilettengängen

Hilfe bei der Nahrungszubereitung

Hilfe bei der Nahrungsaufnahme

Verrichtungen im Haushalt

Spaziergänge

Begleitung bei Arztbesuchen

Medikamentengabe/Insulin

Gespräche/Ansprache

Regelung der finanziellen

Angelegenheiten

$\square$ sonstiges, und zwar:

Wieviele Stunden pro Tag benötigen Sie insgesamt für alle Versorgungstätigkeiten?

etwa Stunden insgesamt pro Tag

5. Wieviel Stunden pro Tag müssen Sie durchschnittlich für Ihren Angehörigen erreichbar, d.h. anwesend, sein?

etwa Stunden insgesamt pro Tag

6. Nehmen Sie Unterstützungsleistungen durch soziale Dienste in Anspruch?

$\square$ nein

$\square$ ja, und zwar:

private Haushaltshilfe

Pflegedienst

Mobilitätsdienst

Zivildienstleistender

fahrbarer Mittagstisch

Tagespflegestätte

mal in der Woche mit jeweils

Stunden

mal in der Woche mit jeweils__ Stunden

mal in der Woche mit jeweils__ Stunden

Krankenwohnung/Kurzzeitpflege mal in der Woche mit jeweils Stunden mal in der Woche mal in der Woche Tage im Jahr 
Wie gut fühlen Sie sich über die folgenden Bereiche informiert? Bitte kreuzen Sie das Kästchen an, das am ehesten auf Sie zutrifft.

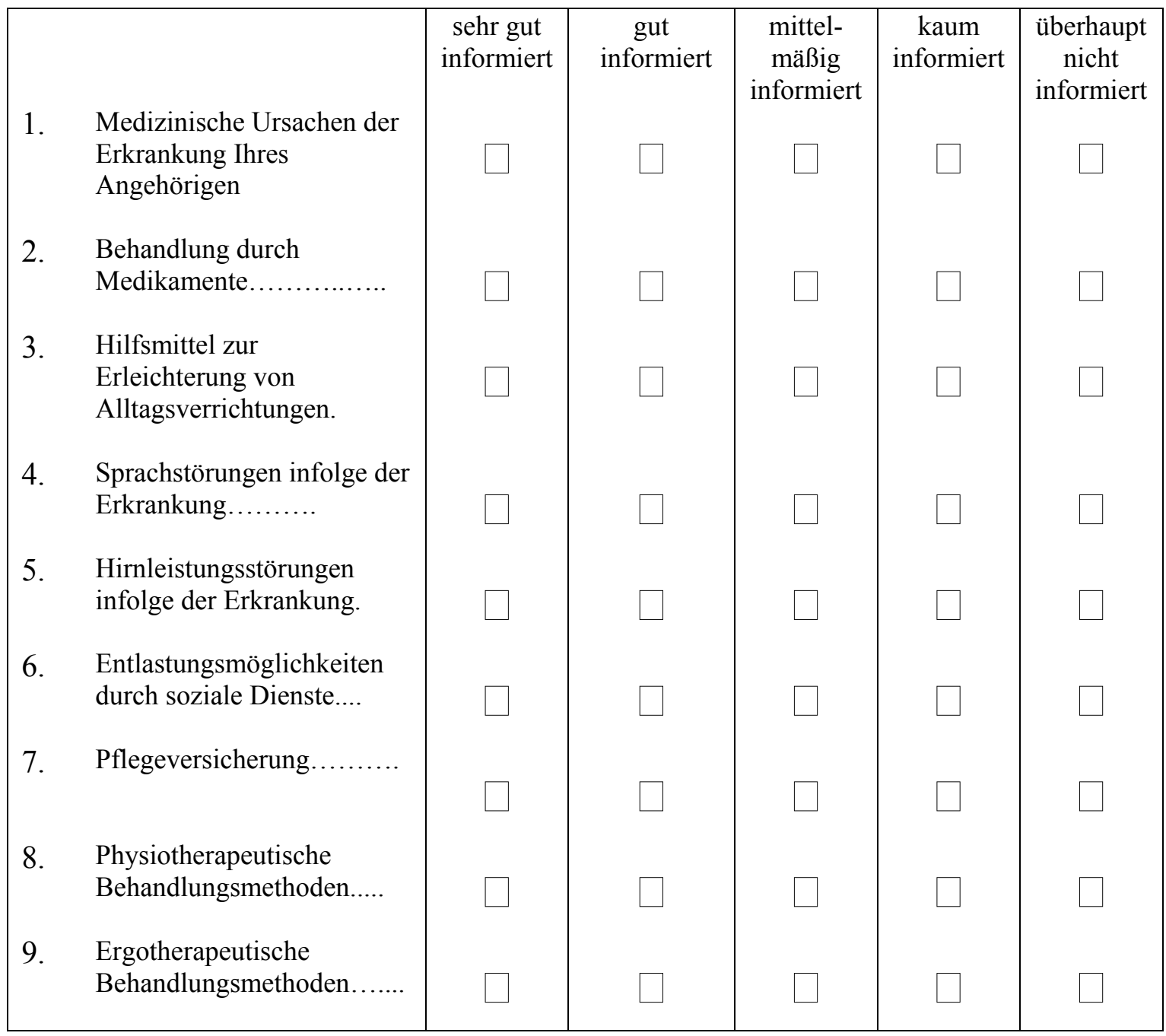

Wie sicher fühlen Sie sich bei Ihrer Arbeit als pflegende(r) Angehörige(r)?

$\begin{array}{cccc}\text { sehr } & \text { ziemlich } & \text { ziemlich } & \text { sehr } \\ \text { unsicher } & \text { unsicher } & \text { sicher } & \text { sicher }\end{array}$


Im Folgenden finden Sie eine Liste von Beschwerden. Bitte geben Sie an, unter welchen Beschwerden Sie leiden. Machen Sie ein Kreuz in eins der vier Kästchen rechts entsprechend der Stärke Ihrer Zustimmung bzw. Ablehnung. Bitte beantworten Sie alle Punkte, lassen Sie keinen aus!

\section{Ich leide unter folgenden Beschwerden:}

1. Kloßgefühl, Enge oder Würgen im Hals.

2. Kurzatmigkeit

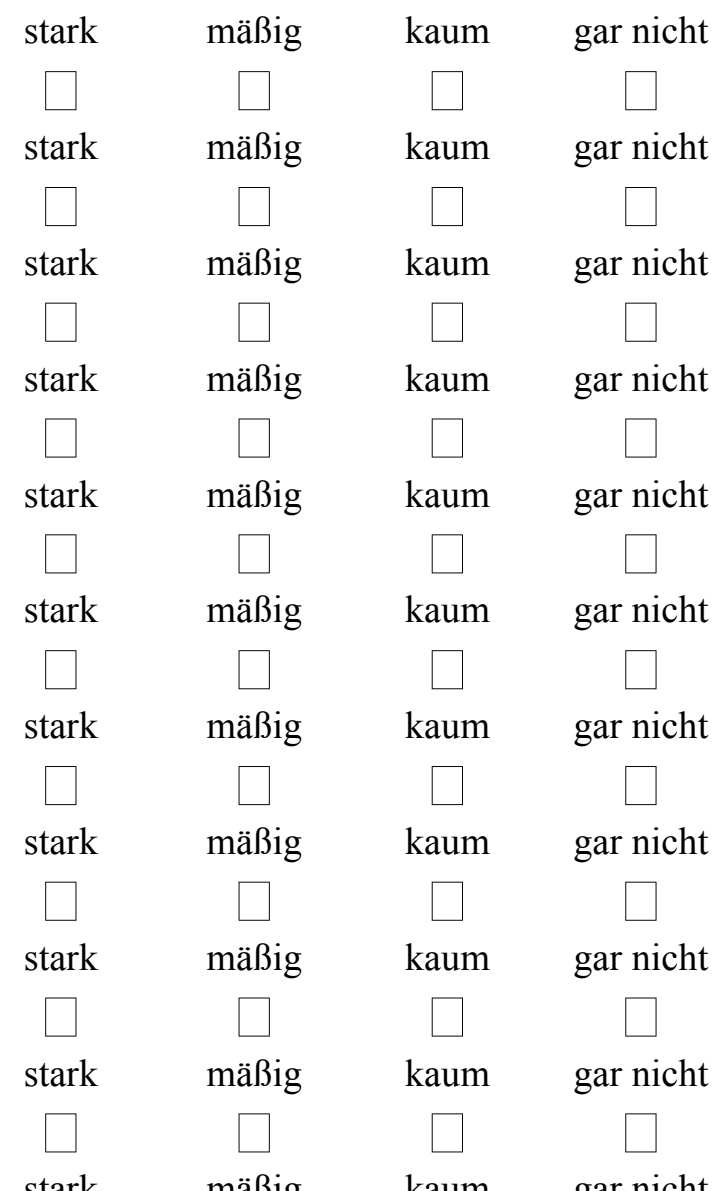

11. Grübelei

stark

12. Starkes Schwitzen

stark

mäßig

kaum

gar nicht

13. Kreuz- oder Rückenschmerzen.

stark

mäßig

kaum

gar nicht

14. Innere Unruhe

stark

mäßig

kaum

gar nicht

15. Schweregefühl bzw. Müdigkeit in den

stark

mäßig

kaum

gar nicht Beinen.

16. Unruhe in den Beinen

stark mäßig kaum gar nicht 
17. Überempfindlichkeit gegen Wärme... stark mäßig kaum gar nicht

18. Überempfindlichkeit gegen Kälte ..... stark mäßig kaum gar nicht

19. Übermäßiges Schlafbedürfnis.......... stark mäßig kaum gar nicht

20. Schlaflosigkeit

21. Schwindelgefühl..................... stark mäßig kaum gar nicht

22. Zittern

23. Nacken- oder Schulterschmerzen...... stark mäßig kaum gar nicht

24. Gewichtsabnahme..................... stark mäßig kaum gar nicht

Bitte geben Sie an, welche Medikamente Sie vorbeugend oder gegen bestimmte Beschwerden in den vergangenen 4 Wochen eingenommen haben:

Medikament gegen Schmerzen

Herz-Kreislauf-Medikament

Beruhigungsmittel

Medikament gegen Infektionskrankheiten (z. B. Erkältung, Magen-Darm-Grippe)

Schlafmittel

Mittel zur Stärkung oder Aktivierung, und zwar:

Sonstiges, und zwar: 
Bitte kreuzen Sie bei den folgenden aussagen die Antwort an, die Ihrem Befinden während der letzten Woche am besten entspricht/entsprochen hat.

Antworten: selten oder überhaupt nicht (weniger als 1 Tag)

manchmal ( 1 bis 2 Tage lang)

öfters ( 3 bis 4 Tage lang)

meistens, die ganze Zeit ( 5 bis 7 Tage lang)

\section{Während der letzten Woche...}

\begin{tabular}{|c|c|c|c|}
\hline $\begin{array}{c}\text { weniger } \\
\text { als 1 Tag }\end{array}$ & $\begin{array}{c}1 \text { bis } 2 \\
\text { Tage }\end{array}$ & $\begin{array}{c}3 \text { bis } 4 \\
\text { Tage }\end{array}$ & $\begin{array}{c}5 \text { bis } 7 \\
\text { Tage }\end{array}$ \\
\hline
\end{tabular}

1. haben mich Dinge beunruhigt, die mir sonst nichts ausmachen.

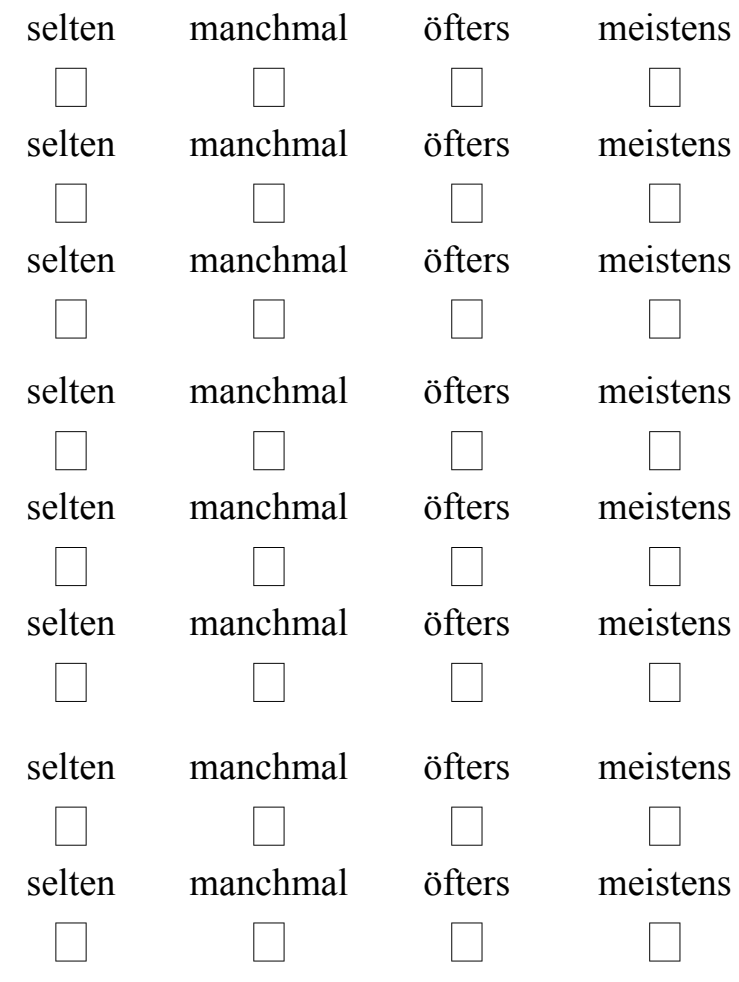

2. hatte ich kaum Appetit.................

3. konnte ich meine trübsinnige Laune nicht loswerden, obwohl mich meine Freunde/Familie versuchten aufzumuntern

4. kam ich mir genauso gut vor wie andere.

5. hatte ich Mühe, mich zu konzentrieren

6. war ich deprimiert/niedergeschlagen...

7. war alles anstrengend für mich.........

8. dachte ich voller Hoffnung an die Zukunft....

9. dachte ich, mein Leben ist ein einziger selten

manchmal

öfters

meistens Fehlschlag

selten

manchmal

öfters

meistens

10. hatte ich Angst....

selten manchmal

öfters

meistens

11. habe ich schlecht geschlafen... 
Während der letzten Woche...

\begin{tabular}{|c|c|c|c|}
\hline $\begin{array}{c}\text { weniger } \\
\text { als 1 Tag }\end{array}$ & $\begin{array}{c}1 \text { bis } 2 \\
\text { Tage }\end{array}$ & $\begin{array}{c}3 \text { bis } 4 \\
\text { Tage }\end{array}$ & $\begin{array}{c}5 \text { bis } 7 \\
\text { Tage }\end{array}$ \\
\hline
\end{tabular}

12. war ich fröhlich gestimmt

selten manchmal

öfters

meistens

13. habe ich weniger als sonst geredet...... selten manchmal öfters meistens

14. fühlte ich mich einsam selten manchmal öfters meistens

15. waren die Leute unfreundlich zu mir... selten manchmal öfters meistens

16. habe ich das Leben genossen............. selten manchmal öfters meistens

17. war ich fröhlich gestimmt

selten selten

18. habe ich weniger als sonst geredet.....

19. fühlte ich mich einsam

20. waren die Leute unfreundlich zu mir...

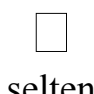

selten

selten manchmal

manchmal

manchmal

manchmal

öfters

meistens

öfters

meistens

öfters

meistens

öfters

meistens

Wie stark fühlen Sie sich durch die Erkrankung Ihres Angehörigen belastet?

$\square$ sehr stark belastet

stark belastet

$\square$ durchschnittlich belastet

wenig belastet

nicht belastet 
Im Folgenden finden Sie 20 Fragestellungen über Ihr Befinden. Bitte lesen Sie jede Aussage sorgfältig durch und entscheiden Sie, wie Sie sich während der letzten 7 Tage gefühlt haben. Entscheiden Sie, welche der folgenden Aussagen auf Sie zutrifft: „selten oder nie“, „manchmal“", „oft"“, oder „meistens oder immer". Kreuzen Sie bitte das entsprechende Kästchen an! Bitte beantworten Sie alle Fragen!

1. Ich fühle mich nervöser und ängstlicher als sonst.

2. Ich fürchte mich ohne jeden Grund

3. Ich rege mich leicht auf oder bekomme das Gefühl, in Panik zu geraten

4. Ich habe das Gefühl zusammenzubrechen

5. Ich glaube, dass alles in Ordnung ist und nichts Schlimmes geschehen wird.

6. Meine Arme und Beine zittern

7. Ich leide an Kopf-, Nacken- und Rückenschmerzen.

8. Ich fühle mich schwach und werde schnell müde

9. Ich fühle mich ganz ruhig und kann gut still sitzen.

10. Ich kann spüren, wie mein Herz ganz schnell pocht...

11. Ich leide unter Schwindelanfällen.

12. Ich habe Ohnmachtsanfälle oder das Gefühl, ohnmächtig zu werden.

13. Ich kann frei ein- und ausatmen

14. Ich bekomme so ein Gefühl von Taubheit und Kribbeln in meinen Fingern und Zehen

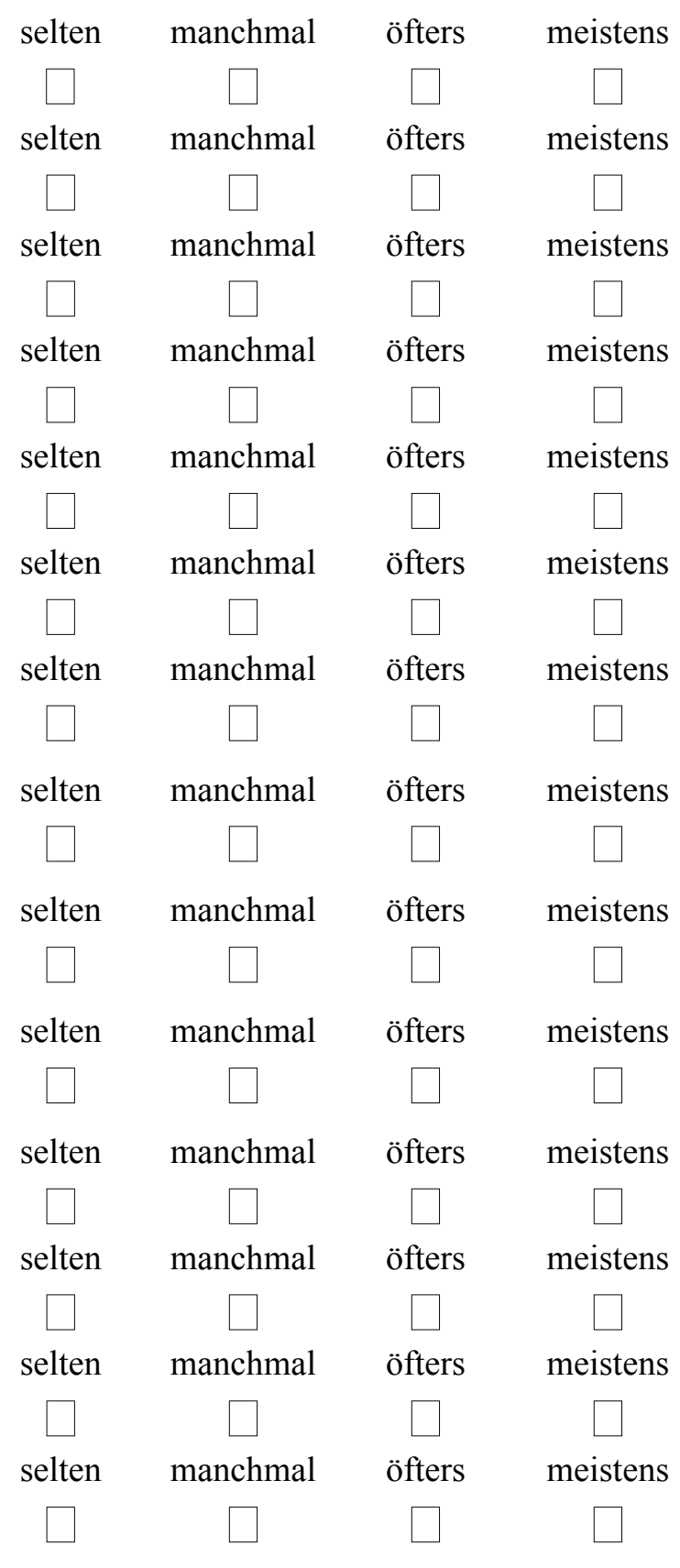


Anhang A

15. Ich leide unter Magenschmerzen oder Verdauungsstörungen.

16. Ich muß häufiger als sonst Wasser lassen.

17. Meine Hände sind gewöhnlich trocken und warm.

18. Ich fühle, wie mein Gesicht heiß wird und ich erröte

19. Ich schlafe leicht ein und finde erholsamen Schlaf.

20. Ich habe Alpträume.

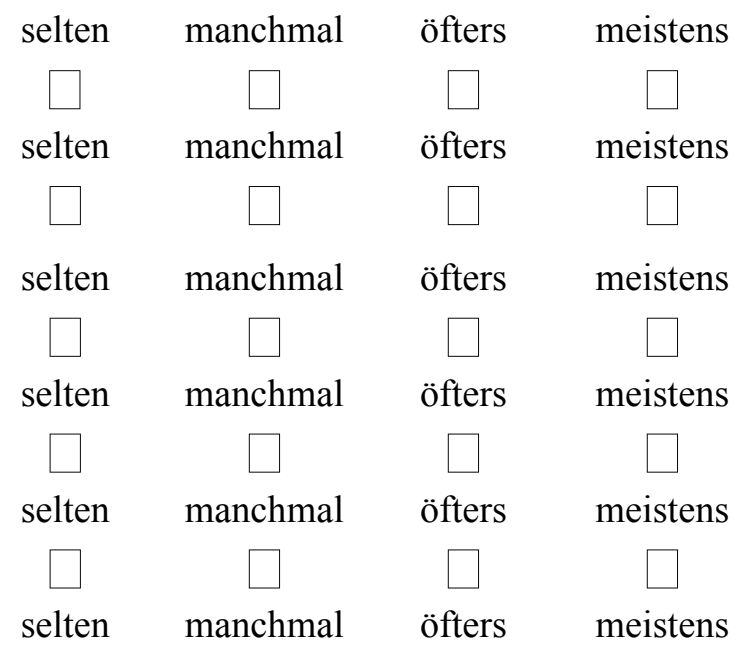


1. Welche Hobbies (Handarbeit, handwerkliche Tätigkeiten, Basteln, Musizieren, Gartenarbeit, Briefmarken o.ä. sammeln etc. ) oder Interessen (Vorträge, Ausflüge, Theater, Sport, Bücher lesen, Kirchgang, Seniorentreff, Enkel hüten etc. ) haben Sie, die Sie noch regelmäßig betrieben?

Bitte zählen Sie alle Ihre Hobbies/Interessen auf:

2. Wie oft verlassen Sie Ihre Wohnung (z. B. für Einkäufe, Erledigungen, Spazierengehen, Arztbesuche, Garten unsw.)?

$$
\begin{aligned}
& \square \text { täglich } \\
& \square \text { mindestens ein- bis zweimal in der Woche } \\
& \square \text { seltener als einmal pro Woche } \\
& \square \text { (fast) nie }
\end{aligned}
$$

3. Wie haben sich in letzter Zeit Ihre Interessen entwickelt?

$$
\begin{aligned}
& \text { habe noch neue Pläne und Interessen } \\
& \text { unverändert } \\
& \text { habe einige Interessen aufgeben müssen } \\
& \text { habe (fast) alle Interessen verloren }
\end{aligned}
$$

4. Sind Sie damit zufrieden wie Sie Ihre Interessen verwirklichen?

voll und ganz, fühle mich nicht beeinträchtigt

$\square$ fühle mich schon eingeschränkt, muß mich damit aber zufrieden geben

$\checkmark$ nein, fühle mich stark eingeschränkt

5. Wie schätzen Sie Ihren Gesundheitszustand im Großen und Ganzen ein?

$$
\begin{aligned}
& \square \text { sehr schlecht } \\
& \square \text { schlecht } \\
& \square \text { mittelmäßig } \\
& \square \text { gut } \\
& \square \text { sehr gut }
\end{aligned}
$$

6. Machen Sie sich Sorgen über Ihre gemeinsame finanzielle zukunft oder über die Ihres Angehörigen?

$$
\begin{aligned}
& \text { selten oder nie } \\
& \text { manchmal } \\
& \text { öfters } \\
& \text { meistens oder immer }
\end{aligned}
$$


Nun geht es darum, wie $\operatorname{Ihr}(\mathrm{e})$ Angehörige in der letzten Woche im Alltag zurecht gekommen ist. Im Folgenden sind verschiedenen Bereiche des täglichen Lebens aufgeführt. Bitte geben Sie zu jedem Bereich an, ob Ihr(e) Angehörige(r) in der letzten Woche diese Tätigkeiten selbständig oder mit Hilfe ausführen konnte. Kreuzen Sie bitte zu jedem Tätigkeitsbereich (z. B. Essen, Waschen) nur ein Kästchen an.

\section{$\underline{\text { 1. Essen }}$}

kann eine Mahlzeit selbständig von einem Tablett oder dem Tisch einnehmen, wenn jemand das Essen in Reichweite stellt; benutzt selbständig Geschirr und Besteck

braucht etwas Hilfe, z. B. beim Fleisch oder Brot schneiden, Butter streichen

kann nicht selbständig essen, auch wenn o.g. Hilfe gewährt wird

\section{Bett/(Roll-)Stuhltransfer}

kann sich ohne Hilfe ins Bett begeben, die Lage wechseln und wieder aufstehen. Beim Gebrauch eines Roostuhls: kann das Bett sicher mit dem Rollstuhl ansteuern, führt den Transfer zum Bett sicher durch, kann aus dem Liegen zu einer sitzenden Position an der Bettkante kommen, kann den Transfer zurück in den Rollstuhl sicher durchführen

es sind geringe Hilfen oder Beaufsichtigung in der einen oder anderen Phase der oben genannten Aktivitäten erforderlich

kann eine sitzende Position ohne Hilfe einnehmen, braucht jedoch erhebliche Hilfe beim Hinlegen, Transfer, Lagewechsel

\section{Waschen}

kann selbständig, ohne Hilfe, Hände und Gesicht waschen, das Haar kämmen, die Zähne putzen, sich rasieren bzw. schminken

ist nicht selbständig bei o.g. Tätigkeiten

\section{Toilettenbenutzung}

Kann Toilette selbständig benutzen, hierbei Kleidung selbständig an- und ausziehen und benutzt selbständig das Toilettenpapier

benötigt Hilfe, z. B. wegen unzureichenden Gleichgewichts oder beim Umgang mit der Kleidung oder bei der benutzung des Toilettenpapiers

Nicht selbständig, in allen Phasen muß Hilfestellung gegeben werden

\section{$\underline{\text { 5. Baden }}$}

kann selbständig in Badewanne oder Dusche ein Vollbad/Duschbad nehmen und sich abseifen, kann alle Handlungen selbst ausführen, ohne daß eine weitere Person anwesend ist

Nicht selbständig bei o.g. Tätigkeit 


\section{Gehen auf Flurebene bzw. Rollstuhlfahren}

Kann mindestens $50 \mathrm{~m}$ gehen ohne Hilfe oder Überwachung, dabei können Hilfsmittel eingesetzt werden (z. B. Unterarmgehstützen, Prothesen), nicht aber Gehwagen

geringe Hilfe oder Überwachung erforderlich, kann mit Hilfsmittel $50 \mathrm{~m}$ gehen

- Kann nicht selbständig gehen, kann aber einen Rollstuhl selbständig bedienen, auch um Ecken herum und an einen Tisch oder ans Bett heranfahren, Strecke mindestens $50 \mathrm{~m}$

Nicht selbständig beim Gehen oder Rollstuhlfahren

\section{Treppensteigen}

kann eine Reihe von Stufen sicher und ohne Hilfe oder Überwachung herauf- oder herabsteigen, dabei dürfen der Handlauf, ein Stock oder Unterarmstützen benutzt werden

benötigt Hilfe oder Überwachung beim Treppensteigen

Nicht selbständig, kann auch mit Hilfe nicht Treppensteigen

\section{An- und Auskleiden}

kann sich selbständig an- und ausziehen (ggf. auch Korsett oder Bruchband), kann Schuschnallen befestigen oder Schnürsenkel binden benötigt Hilfe, kann aber die Hälfte der Tätigkeiten selbständig durchführen

Nicht selbständig, An- und Auskleiden wird zu mehr als der Hälfte von anderer Person durchgeführt

\section{Stuhlkontrolle}

ist ständig kontinent (d. h. kann Stuhl halten)

ist gelegentlich inkontinent, maximal 1 mal pro Woche

ist häufig/ständig inkontinent

\section{Urinkontrolle}

ist Tag und Nacht kontinent (d. h. kann Blasenfunktion kontrollieren), bei Verwendung eines Dauerkatheters kann dieser selbständig versorgt werden ist gelegentlich inkontinent, maximal 1 mal pro Tag

ist häufig/ständig inkontinent

\section{Noch einmal herzlichen Dank für Ihre Mühe!}




\section{Fragebogen zur Bewertung der Angehörigengruppe}

Liebe Teilnehmerinnen und Teilnehmer des Angehörigenkurses,

da wir bemüht sind unseren Angehörigenkurs noch weiter zu verbessern, bitten wir Sie um eine Bewertung des Kurses anhand des vorliegenden Fragebogens. In der Regel sind den einzelnen Aussagen oder Fragen Antwortkästchen zugeordnet. Bitte kreuzen Sie das jeweils für Sie zutreffende Kästchen an.

Damit Ihre Antworten anonym bleiben, bitten wir Sie, zunächst eine Codenummer einzutragen:

\section{Code-Nummer}

Monat Ihrer Geburt in Ziffern (z.B. April=04)

1. Buchstabe des Vornamens Ihrer Mutter (z.B. Anneliese = A)

1. Buchstabe des Vornamens Ihrers Vaters (z.B. Gerd=G)

Den fertig ausgefüllten Fragebogen schicken Sie bitte im beiliegenden Rückumschlag an die Tagesklinik. 


\section{Bitte geben Sie an, inwiefern die folgenden Aussagen auf Ihre Teilnahme am Angehörigenkurs zutreffen:}

\begin{tabular}{|c|c|c|c|c|}
\hline 1. Ich habe neue Informationen erhalten. & $\begin{array}{c}\text { stimmt } \\
\text { nicht } \\
\square\end{array}$ & $\begin{array}{c}\text { stimmt } \\
\text { kaum } \\
\square\end{array}$ & $\begin{array}{c}\text { stimmt } \\
\text { eher } \\
\square\end{array}$ & $\begin{array}{c}\text { stimmt } \\
\text { genau } \\
\square\end{array}$ \\
\hline $\begin{array}{l}\text { 2. Mir ist bewußt geworden, daß ich mit } \\
\text { meiner Situation und meinen Problemen nicht } \\
\text { allein bin. }\end{array}$ & $\begin{array}{c}\text { stimmt } \\
\text { nicht } \\
\square\end{array}$ & $\begin{array}{c}\text { stimmt } \\
\text { kaum } \\
\square\end{array}$ & $\begin{array}{c}\text { stimmt } \\
\text { eher } \\
\square\end{array}$ & $\begin{array}{c}\text { stimmt } \\
\text { genau } \\
\square\end{array}$ \\
\hline $\begin{array}{l}\text { 3. Ich habe nur wenig für mich mitnehmen } \\
\text { können. }\end{array}$ & $\begin{array}{c}\text { stimmt } \\
\text { nicht } \\
\square\end{array}$ & $\begin{array}{l}\text { stimmt } \\
\text { kaum } \\
\square\end{array}$ & $\begin{array}{l}\text { stimmt } \\
\text { eher } \\
\square\end{array}$ & $\begin{array}{c}\text { stimmt } \\
\text { genau } \\
\square\end{array}$ \\
\hline $\begin{array}{l}\text { Mir hat es gut getan, mit anderen } \\
\text { zusammenzusein, die meine Situation } \\
\text { akzeptieren und verstehen. }\end{array}$ & $\begin{array}{c}\text { stimmt } \\
\text { nicht } \\
\square\end{array}$ & $\begin{array}{c}\text { stimmt } \\
\text { kaum } \\
\square\end{array}$ & $\begin{array}{l}\text { stimmt } \\
\text { eher } \\
\square\end{array}$ & $\begin{array}{c}\text { stimmt } \\
\text { genau } \\
\square\end{array}$ \\
\hline $\begin{array}{l}\text { 5. Ich konnte meine eigenen Gefühle und } \\
\text { Meinungen frei äußern. }\end{array}$ & $\begin{array}{c}\text { stimmt } \\
\text { nicht } \\
\square\end{array}$ & $\begin{array}{c}\text { stimmt } \\
\text { kaum } \\
\square\end{array}$ & $\begin{array}{c}\text { stimmt } \\
\text { eher } \\
\square\end{array}$ & $\begin{array}{c}\text { stimmt } \\
\text { genau } \\
\square\end{array}$ \\
\hline 6. Ich konnte mich mit anderen austauschen. & $\begin{array}{c}\text { stimmt } \\
\text { nicht } \\
\square\end{array}$ & $\begin{array}{c}\text { stimmt } \\
\text { kaum } \\
\square\end{array}$ & $\begin{array}{c}\text { stimmt } \\
\text { eher } \\
\square\end{array}$ & $\begin{array}{c}\text { stimmt } \\
\text { genau } \\
\square\end{array}$ \\
\hline 7. Ich fand den Kurs eher langweilig. & $\begin{array}{c}\text { stimmt } \\
\text { nicht } \\
\square \\
\square\end{array}$ & $\begin{array}{c}\text { stimmt } \\
\text { kaum } \\
\square\end{array}$ & $\begin{array}{c}\text { stimmt } \\
\text { eher } \\
\square\end{array}$ & $\begin{array}{c}\text { stimmt } \\
\text { genau } \\
\square\end{array}$ \\
\hline $\begin{array}{l}\text { 8. Die Broschüren und Übersichtspapiere } \\
\text { waren gut verständlich. }\end{array}$ & $\begin{array}{c}\text { stimmt } \\
\text { nicht } \\
\square\end{array}$ & $\begin{array}{c}\text { stimmt } \\
\text { kaum } \\
\square\end{array}$ & $\begin{array}{c}\text { stimmt } \\
\text { eher } \\
\square\end{array}$ & $\begin{array}{c}\text { stimmt } \\
\text { genau } \\
\square\end{array}$ \\
\hline $\begin{array}{l}\text { 9. Ich würde den Kurs anderen betroffenen } \\
\text { Angehörigen weiterempfehlen. }\end{array}$ & $\begin{array}{c}\text { stimmt } \\
\text { nicht } \\
\square\end{array}$ & $\begin{array}{c}\text { stimmt } \\
\text { kaum } \\
\square\end{array}$ & $\begin{array}{c}\text { stimmt } \\
\text { eher } \\
\square\end{array}$ & $\begin{array}{c}\text { stimmt } \\
\text { genau } \\
\square\end{array}$ \\
\hline $\begin{array}{l}\text { 10. Es war gut zu sehen und zu hören, wie } \\
\text { andere mit ihren Problemen fertig werden. }\end{array}$ & $\begin{array}{c}\text { stimmt } \\
\text { nicht } \\
\square\end{array}$ & $\begin{array}{c}\text { stimmt } \\
\text { kaum } \\
\square\end{array}$ & $\begin{array}{c}\text { stimmt } \\
\text { eher } \\
\square\end{array}$ & $\begin{array}{c}\text { stimmt } \\
\text { genau } \\
\square\end{array}$ \\
\hline $\begin{array}{l}\text { 11. Ich habe gelernt, wo ich Hilfen bekommen } \\
\text { kann }\end{array}$ & $\begin{array}{c}\text { stimmt } \\
\text { nicht } \\
\square\end{array}$ & $\begin{array}{c}\text { stimmt } \\
\text { kaum } \\
\square\end{array}$ & $\begin{array}{c}\text { stimmt } \\
\text { eher } \\
\square\end{array}$ & $\begin{array}{c}\text { stimmt } \\
\text { genau } \\
\square\end{array}$ \\
\hline $\begin{array}{l}\text { 12. Ich habe gelernt, besser für mich selbst zu } \\
\text { sorgen. }\end{array}$ & $\begin{array}{c}\text { stimmt } \\
\text { nicht } \\
\square\end{array}$ & $\begin{array}{c}\text { stimmt } \\
\text { kaum } \\
\square\end{array}$ & $\begin{array}{c}\text { stimmt } \\
\text { eher } \\
\square\end{array}$ & $\begin{array}{c}\text { stimmt } \\
\text { genau } \\
\square\end{array}$ \\
\hline $\begin{array}{l}\text { 13. Ich fühle mich jetzt weniger isoliert und } \\
\text { allein. }\end{array}$ & $\begin{array}{c}\text { stimmt } \\
\text { nicht } \\
\square\end{array}$ & $\begin{array}{c}\text { stimmt } \\
\text { kaum } \\
\square\end{array}$ & $\begin{array}{c}\text { stimmt } \\
\text { eher } \\
\square\end{array}$ & $\begin{array}{c}\text { stimmt } \\
\text { genau } \\
\square\end{array}$ \\
\hline
\end{tabular}




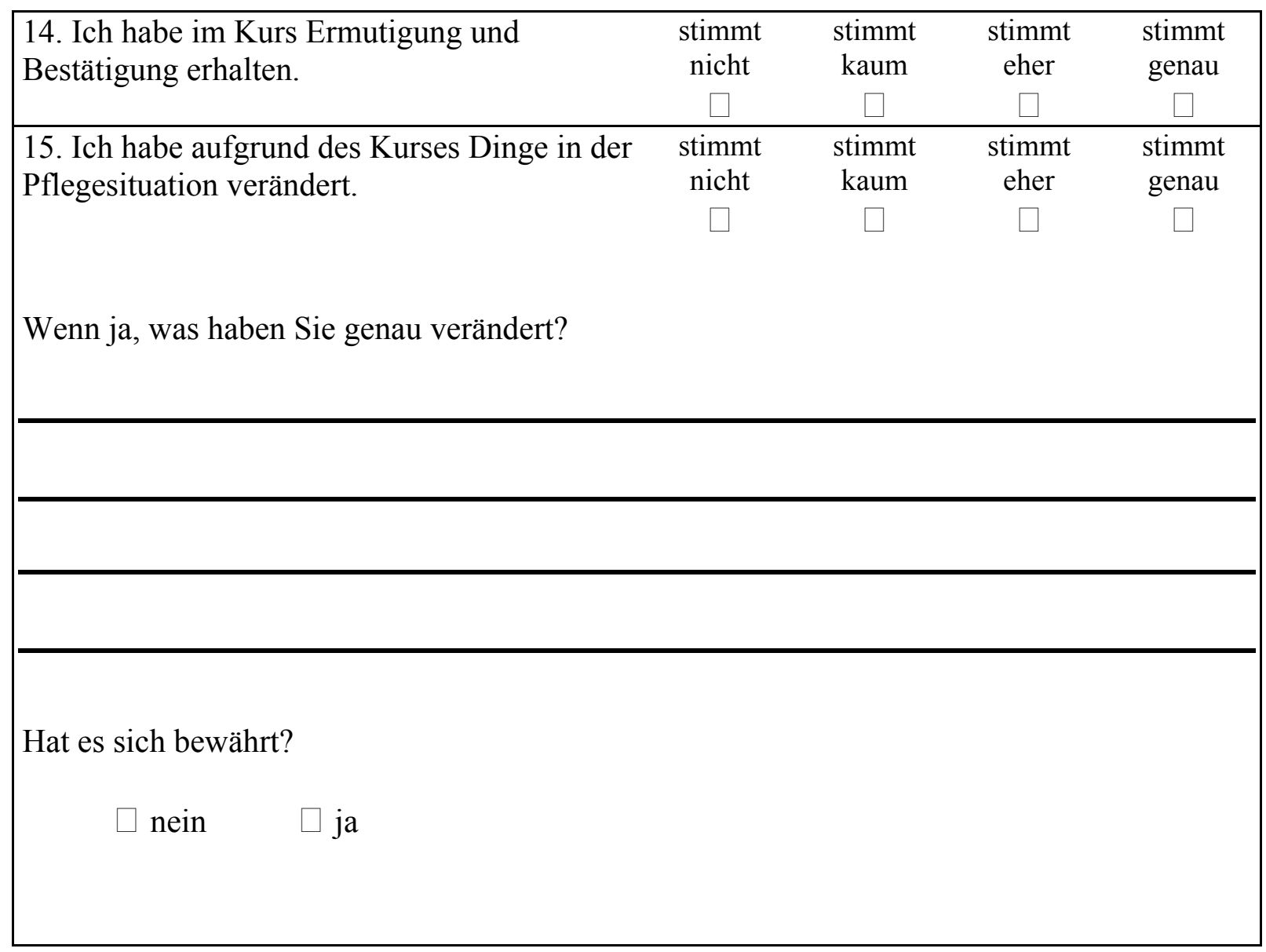


Bitte bewerten Sie nun die einzelnen Kurseinheiten. Falls Sie an einer Kurseinheit nicht teilnehmen konnten, kreuzen Sie bitte bei a) "nein" an und überspringen Sie die Punkte b) bis e).

\section{Medizinische Ursachen des Schlaganfalls und seine Behandlung} (Dr. med. H. Münzberg, Ärztin)

a) Ich habe an der Kurseinheit teilgenommen

b) Die Kurseinheit war verständlich

$\begin{array}{cccc}\text { stimmt } & \text { stimmt } & \text { stimmt } & \text { stimmt } \\ \text { nicht } & \text { kaum } & \text { eher } & \text { genau }\end{array}$

c) Die Kurseinheit hat mir im Alltag stimmt

stimmt

stimmt

stimmt geholfen

nicht

kaum

eher

genau

d) Die Kurseinheit hat zum besseren

stimmt

stimmt

stimmt

stimmt Verständnis meines erkrankten

Angehörigen beigetragen

e) Mir hat gefehlt:

\section{Aktivierende Pflege, Pflegetechniken}

\section{(U. Berndt, Krankenpflegerin)}

a) Ich habe an der Kurseinheit teilgenommen

$\begin{array}{cccc}\text { ja } & \text { nein } & & \\ \square & \square & & \\ \begin{array}{c}\text { stimmt } \\ \text { nicht }\end{array} & \begin{array}{c}\text { stimmt } \\ \text { kaum }\end{array} & \begin{array}{c}\text { stimmt } \\ \text { eher }\end{array} & \begin{array}{c}\text { stimmt } \\ \text { genau }\end{array}\end{array}$

c) Die Kurseinheit hat mir im Alltag geholfen

$\begin{array}{cccc}\begin{array}{c}\text { stimmt } \\ \text { nicht }\end{array} & \text { stimmt } & \text { stimmt } & \text { stimmt } \\ & \text { eher } & \text { genau } \\ \square & \square & \square & \square \\ \text { stimmt } & \text { stimmt } & \text { stimmt } & \text { stimmt } \\ \text { nicht } & \text { kaum } & \text { eher } & \text { genau } \\ \square & \square & \square & \square \\ & & & \\ & & & \end{array}$

d) Die Kurseinheit hat zum besseren Verständnis meines erkrankten Angehörigen beigetragen

e) Mir hat gefehlt: 


\section{Hirnleistungsstörungen nach Schlaganfall}

\section{(A. Barnes, Dipl.-Psychologe)}

a) Ich habe an der Kurseinheit

ja nein teilgenommen

b) Die Kurseinheit war verständlich

$$
\begin{array}{cccc}
\text { stimmt } & \text { stimmt } & \text { stimmt } & \text { stimmt } \\
\text { nicht } & \text { kaum } & \text { eher } & \text { genau }
\end{array}
$$

c) Die Kurseinheit hat mir im Alltag stimmt stimmt nicht kaum

stimmt stimmt geholfen

d) Die Kurseinheit hat zum besseren stimmt stimmt nicht kaum stimmt stimmt Verständnis meines erkrankten Angehörigen beigetragen

e) Mir hat gefehlt:

\section{Der Umgang mit Sprachstörungen}

\section{(R. Bonillo, Sprachtherapeut)}

a) Ich habe an der Kurseinheit

ja nein teilgenommen

b) Die Kurseinheit war verständlich

$$
\begin{array}{cccc}
\text { stimmt } & \text { stimmt } & \text { stimmt } & \text { stimmt } \\
\text { nicht } & \text { kaum } & \text { eher } & \text { genau }
\end{array}
$$

c) Die Kurseinheit hat mir im Alltag

stimmt

stimmt

stimmt stimmt geholfen

d) Die Kurseinheit hat zum besseren

stimmt

stimmt

stimmt

stimmt Verständnis meines erkrankten nicht kaum eher genau Angehörigen beigetragen

e) Mir hat gefehlt: 


\section{Physiotherapeutische Grundlagen, Bobath-Konzept}

(S. van Kaick, Physiotherapeutin)

a) Ich habe an der Kurseinheit

ja nein teilgenommen

b) Die Kurseinheit war verständlich

$\begin{array}{cccc}\text { stimmt } & \text { stimmt } & \text { stimmt } & \text { stimmt } \\ \text { nicht } & \text { kaum } & \text { eher } & \text { genau }\end{array}$

c) Die Kurseinheit hat mir im Alltag stimmt stimmt stimmt stimmt geholfen

d) Die Kurseinheit hat zum besseren

stimmt

stimmt

eher

Verständnis meines erkrankten

nicht

stimmt

stimmt Angehörigen beigetragen

e) Mir hat gefehlt:

\section{Ergotherapeutische Behandlung und Einsatz von Hilfsmitteln}

(A. Nordmann, Ergotherapeut)

a) Ich habe an der Kurseinheit

ja nein teilgenommen

b) Die Kurseinheit war verständlich

$\begin{array}{cccc}\text { stimmt } & \text { stimmt } & \text { stimmt } & \text { stimmt } \\ \text { nicht } & \text { kaum } & \text { eher } & \text { genau }\end{array}$

c) Die Kurseinheit hat mir im Alltag

stimmt

stimmt

stimmt

stimmt geholfen

kaum

eher

genau

d) Die Kurseinheit hat zum besseren

stimmt

stimmt

stimmt

stimmt Verständnis meines erkrankten

nicht

eher genau Angehörigen beigetragen

e) Mir hat gefehlt: 


\section{Finanzielle und soziale Entlastungsmöglichkeiten im Alltag}

\section{(R. Eberhard, Dipl. Sozialarbeiter)}

a) Ich habe an der Kurseinheit

ja nein teilgenommen

b) Die Kurseinheit war verständlich

$$
\begin{array}{cccc}
\begin{array}{c}
\text { stimmt } \\
\text { nicht }
\end{array} & \begin{array}{c}
\text { stimmt } \\
\text { kaum }
\end{array} & \begin{array}{c}
\text { stimmt } \\
\text { eher }
\end{array} & \begin{array}{c}
\text { stimmt } \\
\text { genau }
\end{array}
\end{array}
$$

c) Die Kurseinheit hat mir im Alltag stimmt stimmt nicht kaum

stimmt geholfen

d) Die Kurseinheit hat zum besseren

stimmt stimmt stimmt stimmt Verständnis meines erkrankten nich eher genau Angehörigen beigetragen

e) Mir hat gefehlt:

\section{Belastung der Angehörigen durch die Pflege}

(H. Heier, Dipl.-Psychologin)

a) Ich habe an der Kurseinheit

ja nein teilgenommen

b) Die Kurseinheit war verständlich

$$
\begin{array}{cccc}
\text { stimmt } & \text { stimmt } & \text { stimmt } & \text { stimmt } \\
\text { nicht } & \text { kaum } & \text { eher } & \text { genau }
\end{array}
$$

c) Die Kurseinheit hat mir im Alltag

stimmt

stimmt

stimmt

stimmt geholfen

nich

kaum

eher

genau

d) Die Kurseinheit hat zum besseren

stimmt

stimmt

stimmt

stimmt Verständnis meines erkrankten nicht kaum

eher genau Angehörigen beigetragen

e) Mir hat gefehlt: 


\section{Umgang mit Belastung und Streß}

(H. Heier, Dipl.-Psychologin)

a) Ich habe an der Kurseinheit

ja nein teilgenommen

b) Die Kurseinheit war verständlich

$$
\begin{array}{cccc}
\begin{array}{c}
\text { stimmt } \\
\text { nicht }
\end{array} & \begin{array}{c}
\text { stimmt } \\
\text { kaum }
\end{array} & \begin{array}{c}
\text { stimmt } \\
\text { eher }
\end{array} & \begin{array}{c}
\text { stimmt } \\
\text { genau }
\end{array}
\end{array}
$$

c) Die Kurseinheit hat mir im Alltag

stimmt stimmt nicht

kaum

stimmt

stimmt geholfen

d) Die Kurseinheit hat zum besseren

stimmt

stimmt

eher

genau

Verständnis meines erkrankten

nicht

kaum

stimmt

stimmt

Angehörigen beigetragen

e) Mir hat gefehlt: 
Ich habe folgende Verbesserungsvorschläge für die Gestaltung von zukünftigen Angehörigengruppen:

Mir hat nicht gefallen:

Folgende Dinge fand ich gut und hilfreich:

Vielen Dank für Ihre Mithilfe! 


\title{
Anhang B: Materialien für die qualitative Untersuchung
}

\author{
$\underline{\text { Interviewleitfaden }}$
}

\author{
Informationen für Befragungsteilnehmer (mündlich mitteilen, Merkblatt aushändigen) \\ - Worum geht es? \\ - Inhalte der Befragung \\ - Datenschutz \\ - Verantwortlich für Befragung \\ - Zeitlichen Rahmen klären (evtl. ein 2. Gesprächstermin) \\ -> "Informed consent" einholen \\ -> Aufnahmegerät einschalten
}

\section{Derzeitige Situation (Einstiegsfrage)}

1. Lassen Sie uns beginnen mit Ihrer derzeitigen Alltagssituation. Mich interessiert, welche konkreten Anforderungen und Aufgaben die Pflege oder Unterstützung Ihres Angehörigen für Sie mit sich bringt. Vielleicht können Sie einmal einen typischen Tagesablauf schildern.

2. Hat sich die Situation seit der Reha-Behandlung verbessert?

Wie hat es sich genau verändert?

Ist es so, wie Sie es sich vorgestellt haben?

\section{Soziale Unterstützung}

3. Gibt es jemanden, der Sie bei der Pflege unterstützt?

Haben Sie denjenigen um die Unterstützung gebeten?

Könnten Sie noch andere um Unterstützung bitten?

Was hindert Sie, es zu tun?

4. Was würden Sie sagen, welches sind die 3 Personen, zu denen Sie im Moment die engste Beziehung haben?

Falls der Partner nicht genannt wird, dies ansprechen: Sie haben Ihren Partner jetzt nicht genannt, war das bewusst so?

Hat sich das verändert, im Vergleich zu der Zeit bevor Ihr Angehöriger krank wurde?

Woran liegt das Ihrer Meinung nach?

\section{Frustrationen}

5. Tun Sie im Moment etwas für sich?

Wenn nicht: Macht Ihnen das was aus?

Auf welche Aktivitäten haben Sie verzichten müssen?

6. In welchen Bereichen fühlen Sie sich durch die Pflege und Unterstützung ihres Angehörigen eingeschränkt?

Wie ist das für Sie? 


\section{Gratifikationen}

7. Einen Angehörigen zu pflegen ist belastend und anstrengend. Gibt es aber auch positive Dinge in der Pflege?

\section{Beziehung zum Pflegebedürftigen}

8. Gibt es Veränderungen in der Beziehung zu Ihrem Angehörigen im Vergleich zu früher?

9. Wie würden Sie jetzt die Beziehung zu Ihrem Angehörigen beschreiben?

\section{Motivation zur Pflege}

10. War es für Sie selbstverständlich, die Pflege Ihres Angehörigen zu übernehmen? Was waren die Hauptgründe?

Gab es andere Personen, die das von Ihnen erwartet haben, dass Sie die Pflege übernehmen?

\section{Coping-Strategien}

11. Gibt es in der derzeitigen Situation etwas, das Ihnen Trost gibt, oder Ihnen hilft, die Situation zu bewältigen?

12. Was tun Sie, wenn es Ihnen sehr schlecht geht?

Können Sie ein Beispiel nennen für eine konkrete schwierige Situation, und was Sie in der Situation gemacht haben?

\section{Motivation zur Teilnahme an der Angehörigengruppe}

\section{a) Bei Teilnehmern am Kurs:}

13.(TG) Sie haben vor einiger Zeit am Angehörigenkurs teilgenommen, der in der Tagesklinik angeboten wurde.

Welche Gründe hatten Sie an dem Angehörigenkurs teilzunehmen?

\section{b) Bei Personen aus der Kontrollgruppe:}

13. (KG) In der Tagesklinik gibt es ja Kursangebote und Gesprächsgruppen für pflegende Angehörige. Würde Sie eine Teilnahme an einem derartigen Angebot interessieren?

14. (KG) Was sind für Sie Hinderungsgründe daran teilzunehmen?

Falls Grund angeführt wird, dass Patient nicht allein zuhause bleiben kann:

Wenn Sie die Möglichkeit hätten, Ihren Angehörigen mitzubringen, so dass er in der Zeit in einem anderen Raum anderweitig beschäftigt wird, würden Sie das Angebot eher in Anspruch nehmen?

\section{Wirkung des Angehörigenkurses/der Angehörigengruppe (Nur für Teilnehmer)}

14. Wie haben Sie den Kurs erlebt?

15. Welche speziellen Anregungen hat Ihnen der Angehörigenkurs gebracht? 
16. Hat der Angehörigenkurs Sie dazu gebracht, etwas bestimmtes in Ihrer Pflegesituation zu verändern?

Wenn ja, was?

17. Wie haben Sie die anderen Teilnehmer in dem Kurs erlebt?

18. Nach dem Kurs haben Sie sich entschlossen auch weiter an einer Gesprächsgruppe teilzunehmen. Was hat Sie dazu bewogen?

19. Welche Anregungen oder Unterstützungen bringt Ihnen die Angehörigengruppe?

20. Haben die Dinge, die Sie in der Gruppe erfahren, Auswirkungen auf Ihren Alltag als pflegende Angehörige?

21. Wie steht Ihr Angehöriger dazu, dass Sie in die Angehörigengruppe kommen?

Worauf ist seine Reaktion Ihrer Meinung nach zurückzuführen?

22. Was war das wichtigste, das Sie aus der Angehörigengruppe herausgezogen haben?

23. Haben Sie Vorschläge für Veränderungen für die Angehörigengruppe?

Möchten Sie zu bestimmten Themen etwas hören?

Gibt es Dinge, die die Leiterin anders machen soll?

24. Was wäre ein Grund für Sie nicht mehr in die Angehörigengruppe kommen?

\section{Abschluß}

26. Wie war das Gespräch hier für Sie, wie haben Sie sich gefühlt? 


\section{Kurzinfo für die Interviewerin}

Name, Adresse:

Alter der Angehörigen:

Alter des Patienten:

Erkrankung des Patienten:

Funktionelle Einschränkungen des Patienten:

Besonderheiten der Pflegesituation:

Teilnahme am Kurs/Gesprächsgruppe (Zeitraum): 
$\underline{\text { Kurzbericht zur Nachbereitung des Interviews }}$

Beginn (Uhrzeit):

Ende (Uhrzeit):

Das Interview fand statt:

$\square$ zuhause

$\square$ im EGZB

Bereitschaft der Angehörigen zum Gespräch:

$\square$ bereitwillige Teilnahme

$\square$ zögerliche Teilnahme

Eindruck von der Angehörigen im Gespräch

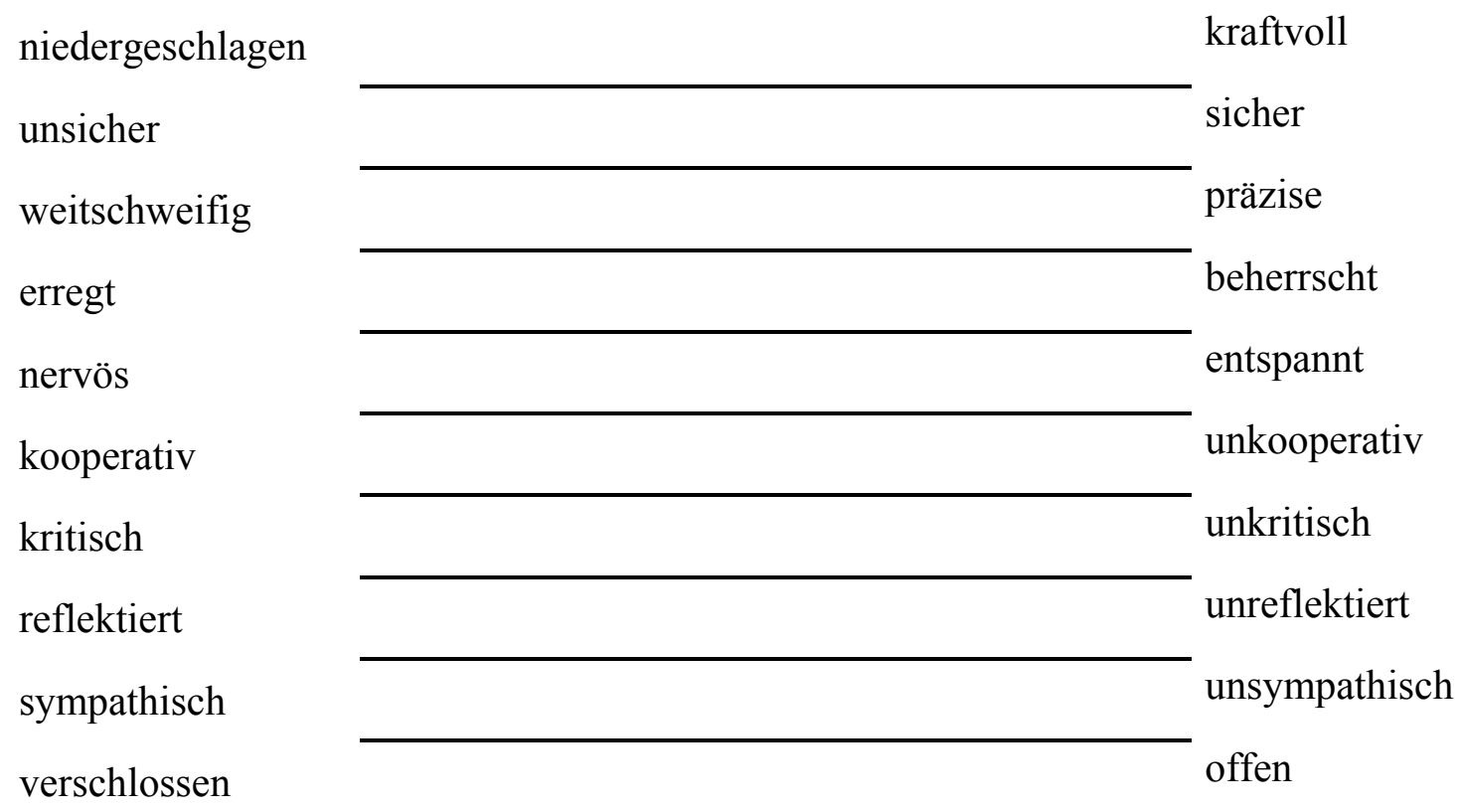

Allgemeines subjektives Erleben der Interviewerin im Gespräch

unsicher sicher

ohne Zeitdruck unter Zeitdruck

gehemmt locker

entspannt nervös 


\section{Sonstige Anmerkungen/ Besonderheiten in der Interviewsituation}

\section{Leitfragen:}

1. Gab es Dinge, die die Angehörige äußerte, nachdem das Band abgeschaltet war, die relevant sein könnten für die Auswertung?

2. Gab es „Ungereimtheiten“ oder Widersprüche in den Aussagen der Interviewpartnerin?

3. Gab es Stellen im Gespräch, die etwas besonderes bei Ihnen als Interviewerin ausgelöst haben (Nervosität, Ungeduld, Unbehagen, Traurigkeit, etc.)?

4. Gibt es sonst noch etwas, das relevant sein könnte für die Auswertung? 


\section{Transkriptionsregeln für die Angehörigen-Interviews}

\section{Anonymisierung}

Alle Nachnamen wurden abgekürzt.

\section{Umschrift}

Die Interviews sind wortwörtlich wiedergegeben. Alle Äußerungen wurden in möglichst lesbarer Weise erfasst. Wörter wurden in der Regel nach orthografischen Konventionen des Hochdeutschen geschrieben. Abweichungen von der hochdeutschen Lautung wurden in wortwörtlicher Umschrift wiedergegeben. Die Satzzeichen wurden als Lesehilfen gesetzt ohne strenge Beachtung der Dudenregeln für Interpunktion.

Um den Sinnzusammenhang leichter verständlich zu machen, wurden bei Bedarf folgende Sonderzeichen eingefügt:

\section{Satzabbruch}

Ein Satzabbruch mit nachfolgend neuem Satzbeginn wurde mit einem doppelten Schrägstrich (//) gekennzeichnet.

\section{Ausgefallene Buchstaben}

Ausgefallene Buchstaben wurden innerhalb eines Wortes durch ein Apostroph als Lesehilfe ersetzt, wenn es der besseren Lesbarkeit diente. Am Ende des Wortes wurden auf Apostrophe verzichtet, um eine flüssigere Lesbarkeit zu gewährleisten.

\section{Kürzungen}

„(...)“ markiert Satzteile, die für die aufgeführte Textpassage nicht relevant sind.

\section{Simultanpassagen}

In „\#“ gesetzte Satzteile wurden von den beiden Gesprächspartnerinnen gleichzeitig geäußert.

\section{Unverständliche bzw. unsicher verstandene Passagen}

Unverständliche Wörter oder Passagen wurden durch eine Anmerkung in Klammern

z. B. (EIN WORT UNDEUTLICH) oder (SATZ UNDEUTLICH) gekennzeichnet.

\section{Kommentare}

Kursiv gedruckte Anmerkungen wurden eingefügt, um unklare Äußerungen näher zu erläutern. Nonverbale Handlungen (WEINEN, SEUFZEN) erscheinen in Klammern in Großbuchstaben.

\section{Betonungen}

In der gesprochenen Sprache betonte Worte wurden in Großbuchstaben gesetzt. 